\title{
Corrigendum to: Why are Ionic Liquids Liquid? A Simple Explanation Based on Lattice and Solvation Energies.
}

[J. Am. Chem. Soc., 2006, 128, 13427-13434]. Ingo Krossing, John M. Slattery, Corinne Daguenet, Paul J. Dyson, Alla Oleinikova and Hermann Weingärtner.

A reader of our recent article spotted a sign error in one of our equations that requires us to make a correction to our original publication; we apologize for any confusion caused by this mistake. On page 13429 , figure 1 . The signs of $\Delta_{\text {solv }} G^{T}$ should be positive, rather than negative. This reflects the fact that $\mathrm{IL}_{(g)}$ to $\mathrm{IL}_{(l)}$ is an exergonic process and $\Delta_{\text {solv }} G^{T}$ already has a negative value. A revised figure 1 is shown below. This change does not affect our calculations of the standard free energy of fusion $\left(\Delta_{f u s} G^{\mathrm{O}}\right)$ in table 2, as we intuitively used the correct signs in these calculations.

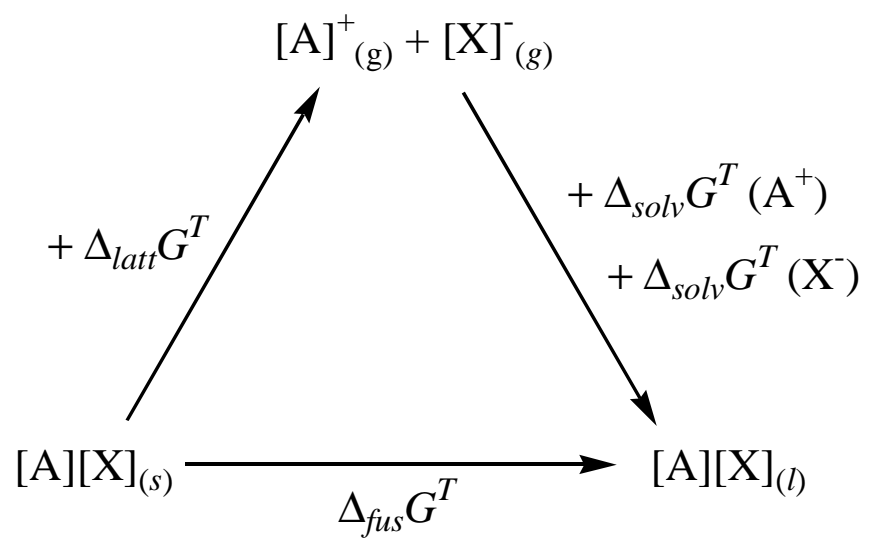

Revised Figure 1 of the original publication. Born-Fajans-Haber cycle for the assessment of the melting (fusion) of a binary salt composed of complex ions ([A][X]), at temperature $T$, from lattice and solvation energies. 
This error is also present in the cycle shown on page 13433, figure 2 , but in this case we need to reassess the conclusions drawn from this cycle. When the correct signs are used to derive equations 6 to 10 (see below) it is clear that figure 2 as it appears in the original publication is over simplified and cannot be used to estimate $\Delta_{\text {solv }} G^{T}$ as previously described. Since substitution of (9) into (10) gives $\Delta_{f u s} G^{T}=\Delta_{f u s} G^{298}$, which is not physically meaningful.

$$
\begin{array}{cc}
\Delta_{\text {fus }} G^{298}=\Delta_{\text {latt }} G^{298}+\Delta_{\text {solv }} G^{298} & \text { (Incorrect in the original paper) (6) } \\
\Delta_{\text {fus }} G^{T}=\Delta_{\text {latt }} G^{T}+\Delta_{\text {solv }} G^{T} & \text { (Incorrect in the original paper) (7) } \\
\Delta_{\text {solv }} G^{T}=\Delta_{\text {solv }} G^{298}-\Delta_{\text {corr }} G^{T} & \text { (Incorrect in the original paper) (8) } \\
\Delta_{\text {corr }} G^{T}=\Delta_{\text {latt }} G^{T}-\Delta_{\text {latt }} G^{298} & \text { (Correct in the original paper)(9) } \\
\Delta_{\text {fus }} G^{T}=\Delta_{\text {latt }} G^{T}+\Delta_{\text {solv }} G^{298}-\Delta_{\text {corr }} G^{T} & \text { (Incorrect in the original paper) (10) }
\end{array}
$$

Figure 2 as it appears in the original publication (and equations 8, 9 and 10 derived from it including those shown above) are incorrect because the temperatures of $[\mathrm{A}][\mathrm{X}]_{(\mathrm{s})}$ and $[\mathrm{A}][\mathrm{X}]_{(\mathrm{l})}$ were not included in the cycle. It is this oversimplification that leads to the curious result $\Delta_{f u s} G^{T}=$ $\Delta_{f u s} G^{298}$. We present here a revised figure 2, which relates two Born-Fajans-Harber cycles at different temperatures. The first (shown on the left) occurs under standard conditions and is used to calculate $\Delta_{f u s} G^{298}$. The second (on the right) takes place at temperature $\mathrm{T}$ and is used to calculate $\Delta_{f u s} G^{T}$. 


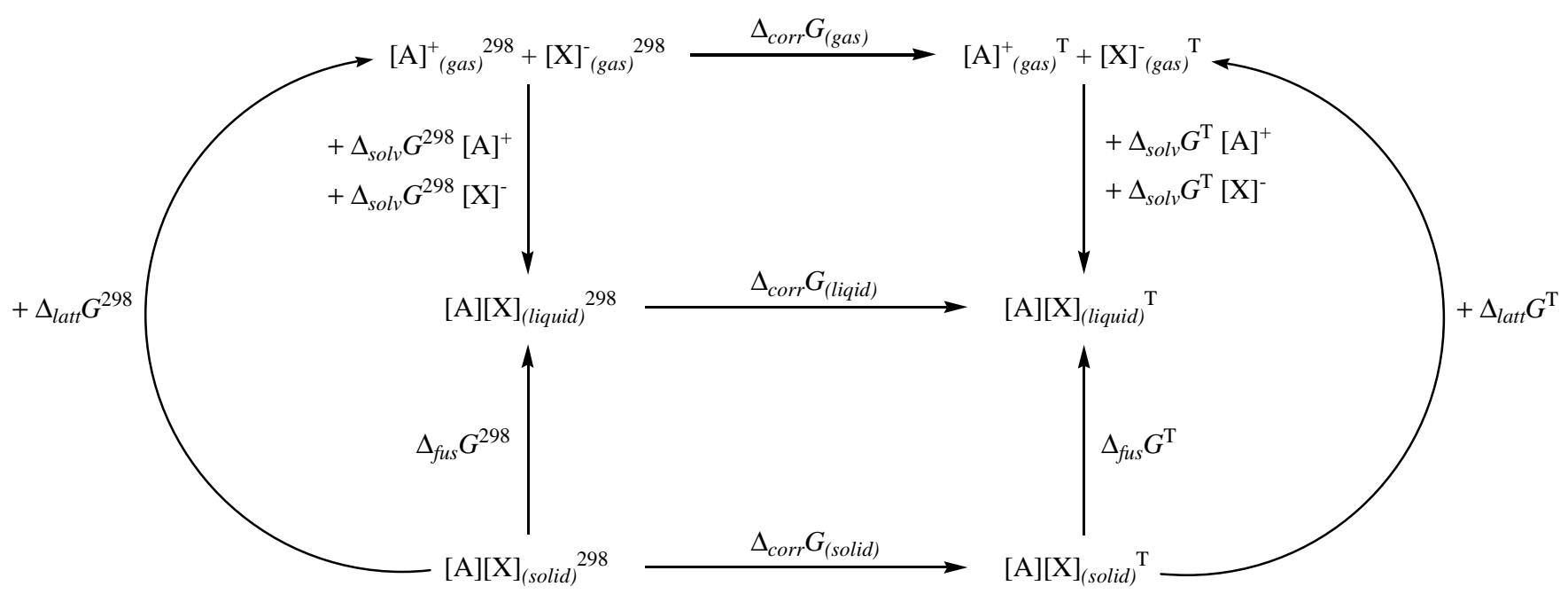

Revised Figure 2 of the original publication. Born-Fajans-Haber cycle showing the relationship between two cycles based on figure 1. The first cycle takes place under standard conditions $\left(298.15 \mathrm{~K}, 10^{5} \mathrm{~Pa}\right)$ and the second at temperature $\mathrm{T}\left(10^{5} \mathrm{~Pa}\right)$.

In the original publication we use the cycle shown in the original figure 2 to estimate $\Delta_{\text {solv }} G^{T}$ and thus calculate $\Delta_{f u s} G^{T}$ at different temperatures. Unfortunately, when considering the revised figure 2 (above) it is evident that we cannot use such an approach, as we are unable to calculate $\Delta_{\text {corr }} G_{(\text {liquid })}$, which would allow us to approximate $\Delta_{\text {solv }} G^{T}$ from our calculations at $298.15 \mathrm{~K}$.

We are currently working on a method to estimate $\Delta_{\text {corr }} G_{(\text {liquid })}$ and thus $\Delta_{\text {solv }} G^{T}$ by estimation of the entropy of the liquid IL using a new VBT equation. However, it is beyond the scope of this corrigendum to discuss this ongoing work. Thus, we suggest that in order to use the method described in the original publication to predict the melting points and dielectric constants of ILs one should make the approximation that $\Delta_{\text {solv }} G^{T}=\Delta_{\text {solv }} G^{298}$ and calculate $\Delta_{f u s} G^{T}$ using equation 11 (below). This is not an unreasonable approximation, at least at temperatures close to $298.15 \mathrm{~K}$, since it appears that the dielectric constant of these ILs does not vary a great deal with temperature (at least at temperatures close to $298 \mathrm{~K}$ ) - see original manuscript for details. However, this will introduce an error due to the temperature dependent entropy term in $\Delta_{\text {solv }} G^{T}$. 


$$
\begin{gathered}
\text { S-4 } \\
\Delta_{\text {fus }} G^{T}=\Delta_{\text {latt }} G^{T}+\Delta_{\text {solv }} G^{298}
\end{gathered}
$$

This new approximation means that our predictions in table 4 need to be amended (the supplementary information has been updated to take into account this change) [please see the original publication for references included in this table]. 
S-5

Revised Table 4 of the original publication. Melting point and dielectric constant predictions for the ILs in this study.

\begin{tabular}{|c|c|c|c|c|c|c|}
\hline Salt & $\begin{array}{l}\text { Experimental } \\
\text { Melting } \\
\text { Point }\left({ }^{\circ} \mathrm{C}\right)\end{array}$ & $\begin{array}{l}\text { Predicted } \\
\text { Melting } \\
\text { Point } \\
\left({ }^{\circ} \mathrm{C}\right)\end{array}$ & $\begin{array}{l}\text { Predicted } \\
\text { Melting } \\
\text { Point } \\
\text { (corrected) } \\
\left({ }^{\circ} \mathrm{C}\right)\end{array}$ & $\begin{array}{l}\text { Experimental } \\
\text { Dielectric } \\
\text { Constant }\end{array}$ & $\begin{array}{l}\text { Predicted } \\
\text { Dielectric } \\
\text { Constant }\end{array}$ & $\begin{array}{l}\text { Predicted } \\
\text { Dielectric } \\
\text { Constant } \\
\text { (corrected) }\end{array}$ \\
\hline$[\mathrm{EMIM}]\left[\mathrm{BF}_{4}\right]^{2,69}$ & 15 to $-1^{t}$ & -21 & 11 & 12.9 & 10.0 & 13.5 \\
\hline$\left[\right.$ EMIM] $[\mathrm{TfO}]^{2,64,70}$ & -9 to $-15^{\ddagger}$ & -55 & -20 & 15.1 & 10.0 & 14.0 \\
\hline$[\mathrm{EMIM}]\left[\mathrm{Tf}_{2} \mathrm{~N}\right]$ & -19 & -43 & -6 & 12.3 & 9.5 & 14.5 \\
\hline$\left[\mathrm{C}_{3} \mathrm{MIM}\right]\left[\mathrm{Tf}_{2} \mathrm{~N}\right]$ & $\begin{array}{l}\text { Not } \\
\text { observed. }\end{array}$ & -43 & -6 & 11.8 & * & $*$ \\
\hline$[\mathrm{BMIM}]\left[\mathrm{BF}_{4}\right]^{47}$ & $\begin{array}{l}\text { Not } \\
\text { observed. }\end{array}$ & -47 & -11 & $11.7^{\ddagger}$ & $*$ & $*$ \\
\hline$[\mathrm{BMIM}]\left[\mathrm{PF}_{6}\right]^{47}$ & 9 & -20 & 15 & $11.4^{\ddagger}$ & 8.5 & 11.5 \\
\hline [BMIM][TfO] & 13 & -65 & -26 & 13.2 & 7.0 & 9.0 \\
\hline$[\mathrm{BMIM}]\left[\mathrm{Tf}_{2} \mathrm{~N}\right]$ & -5 & -49 & -11 & 11.6 & 7.5 & 11.0 \\
\hline$[\mathrm{BMMIM}]\left[\mathrm{Tf}_{2} \mathrm{~N}\right]$ & $\begin{array}{l}\text { Not } \\
\text { observed. }\end{array}$ & -49 & -10 & 11.5 & $*$ & $*$ \\
\hline$\left[\mathrm{C}_{5} \mathrm{MIM}\right]\left[\mathrm{Tf}_{2} \mathrm{~N}\right]$ & -10 & -55 & -15 & 11.4 & 7.5 & 10.5 \\
\hline$[\mathrm{BPy}]\left[\mathrm{Tf}_{2} \mathrm{~N}\right]$ & 23 & -41 & -2 & 11.5 & 6 & 8.5 \\
\hline$[\mathrm{BMPyr}]\left[\mathrm{Tf}_{2} \mathrm{~N}\right]$ & -9 & -42 & -2 & 11.9 & 8.5 & 13.5 \\
\hline$\left[\mathrm{C}_{5} \mathrm{MPyr}\right]\left[\mathrm{Tf}_{2} \mathrm{~N}\right]$ & 8 & -41 & 0 & 11.1 & 7.0 & 10.0 \\
\hline$\left[\mathrm{C}_{5} \mathrm{NEt}_{3}\right]\left[\mathrm{Tf}_{2} \mathrm{~N}\right]$ & 0 & -32 & 7 & 10.0 & 7.0 & 11.0 \\
\hline
\end{tabular}

Values not determined during this study, relevant references are indicated next to compound names.

*It is not possible to make dielectric constant predictions, if the melting point is not known; so no predictions are made for salts where no melting point was observed. 
The predicted melting points and dielectric constants are all lower than in the original publication, as the correction factor $\Delta_{c o r r} G$ is no longer included in the calculations. The predictions are now $24-49{ }^{\circ} \mathrm{C}$ lower than the experimental values (excluding the two problem cases: $[\mathrm{BMIM}][\mathrm{TfO}]$ and $[\mathrm{BPy}]\left[\mathrm{Tf}_{2} \mathrm{~N}\right]$ described in the original publication). However, when the predicted and experimental data are plotted against each other (see revised supplementary information) they are relatively well correlated (gradient $=0.97$, intercept $=-28.15, \mathrm{r}^{2}=0.58$ ), suggesting a systematic error in the calculations. The predicted and experimental melting points here are actually better correlated than those described in the original paper, albeit with an apparent systematic underestimation.

Since it is necessary to make several approximations when calculating $\Delta_{f u s} G^{T}$, the apparent systematic error in these predictions contains contributions from several sources. However, it is likely that the omission of dispersive interactions in the VBT equation used to calculate $U_{P O T}$ for these salts (which only accounts for Coulombic interactions between ions) is a major component of this and may lead to an underestimation of the lattice enthalpies of these ILs. Since dispersive interactions become a more substantial component of the lattice enthalpy for large ions (i.e. those found in ILs), where Coulombic interactions between ions are considerably reduced, this may have a significant effect on these calculations. An alternative method for calculating the lattice enthalpies of salts from their molecular volumes has been proposed by Bartlett et al. ${ }^{1}$ In a personal communication between N. Bartlett and J. Passmore, N. Bartlett estimates the effects of dispersive interactions on the lattice enthalpies calculated using this method at $+42 \mathrm{~kJ} \mathrm{~mol}^{-1}$. $^{2}$ Experimental thermodynamic measurements that allow the calculation of lattice enthalpies are desirable to investigate the magnitude of this effect for ILs. ${ }^{3-6}$

In order to facilitate the use of this method for the prediction of melting points and dielectric constants of ILs we have explored the possibility of using an empirical correction factor to 
account for the apparent systematic errors in these calculations. This correction factor (in $\mathrm{kJ} \mathrm{mol}^{-}$

${ }^{1}$ ) was applied to the final $\Delta_{f u s} G^{T}$ value, rather than to any specific component, as it accounts for errors from several sources. For each salt (excluding the two problem cases described in the original publication) the correction factor required to accurately predict the experimentally determined melting point was calculated (see supplementary information). When this was plotted against $V_{m}$ for these ILs a reasonable correlation was observed $\left(\mathrm{r}^{2}=0.45\right.$ - see revised supplementary information), which allows us to propose the following empirical equation for deriving the correction factor from the molecular volume of the salt:

$$
\Delta_{\text {corr }} G=45 \mathrm{kJmol}^{-1} \mathrm{~nm}^{-3} \cdot V_{m}+5 \mathrm{kJmol}^{-1}
$$

Melting points and dielectric constant predictions using this new correction factor are shown in the revised table 2 . The corrected predictions are in very good agreement with the experimental data $\left(s_{e s t}=8{ }^{\circ} \mathrm{C}\right.$ and 1.3 units respectively - not including the two problem cases) and the correlation between observed and predicted melting points is better than the previously published predictions $\left(\right.$ gradient $=0.90$, intercept $\left.=27.60, \mathrm{r}^{2}=0.60\right)$. Although more work in this area is desirable in order to determine if this correction scheme is applicable to all ILs.

We sincerely apologize for this error and would like to reiterate that the underlying cycle shown in figure 1 is correct and that it would be possible to calculate $\Delta_{f u s} G$ exactly, if $\Delta_{\text {latt }} G$ and $\Delta_{\text {solv }} G$ were also known accurately. It is only the approximations that we must make in order to calculate the latter two values in a simple manner that leads to small errors in the calculated $\Delta_{f u s} G$ values. Further work in this area will allow these calculations to be refined and $\Delta_{f u s} G$ to be calculated with greater accuracy, and perhaps yet more simply.

ACKNOWLEDGMENT: We would like to thank Dr. Ian Brotherston for his help in identifying the error in our original publication. 


\section{References.}

(1) Mallouk, T. E.; Rosenthal, G. L.; Muller, G.; Busasco, R.; Bartlett, N. Inorg. Chem. 1984, 23, 3167-3173.

(2) Bartlett, N. Personal Communication to Jack Passmore.

(3) Emel'yanenko, V. N.; Verevkin, S. P.; Heintz, A. J. Am. Chem. Soc. 2007, ASAP Article.

(4) Zhang, Z-H.; Tan, Z-C.; Sun, L-X.; Yang, J-Z.; Lv, X-C.; Shi, Q. Thermochim. Acta 2006, 141-146.

(5) Kabo, G. J.; Blokhin, A. V.; Paulechka, Y. U.; Kabo, A. G.; Shymanovich, M. P.; Magee, J. W. J. Chem. Eng. Data 2004, 49, 453-461.

(6) Blokhin, A. V.; Paulechka, Y. U.; Kabo, G. J. J. Chem. Eng. Data 2006, 51, 1377-1388. 
S-9

\section{Why are Ionic Liquids Liquid? - Revised Supplementary Information to the original manuscript}

\section{Experimental details:}

All chemicals, 1-bromoethane (Fluka, >98\%), 1-chloropropane (Acros, 99\%), 1-chlorobutane (Acros, >99\%), 1-bromopentane (Acros, 98\%), 1-methylpyrrolidine (Acros, 98\%), pyridine (Acros, >99\%), triethylamine (Merck, for synthesis), $\mathrm{LiTf}_{2} \mathrm{~N}$ (Fluka, $>99 \%$ ), $\mathrm{KPF}_{6}$ (Lancaster, 98\%), $\mathrm{NaBF}_{4}$ (Lancaster, 97\%), methyltrifluoromethanesulfonate (Acros, 96\%) were used as received apart from 1-methylimidazole (Lancaster, 99\%), 1-butylimidazole (Lancaster, 99\%) and 1, 2-dimethylimidazole (Acros, 98\%), which were distilled from $\mathrm{KOH}$ prior to use. The halide salts of imidazolium, pyrrolidinium, pyridinium and ammonium cations were prepared adapting literature procedures. ${ }^{\mathrm{i}, \mathrm{ii}}$ The hydrophobic $\mathrm{Tf}_{2} \mathrm{~N}^{-}$and $\mathrm{PF}_{6}^{-}$based ionic liquids were synthesised via metathesis in water at room temperature, according to published methods. ${ }^{\text {iii,iv }}$ All the ionic liquids were carefully washed with water to avoid the presence of halides impurities and then dried under vacuum at $70^{\circ} \mathrm{C}$ for several hours. ${ }^{\mathrm{v} \text {, iv }}$ The [bmim][TfO] ionic liquid was prepared by direct methylation of 1 -butylimidazole ${ }^{\mathrm{vi}}$ and the $\left[\mathrm{bmim}^{\mathrm{B}}\right]\left[\mathrm{BF}_{4}\right]$ by metathesis in dichloromethane $\mathrm{i}^{\mathrm{i}}$ following literature procedures. NMR spectra were recorded on a Bruker Avance 400 spectrometer and ${ }^{1} \mathrm{H}$ chemical shifts were referenced to residual solvent.

1-ethyl-3-methylimidazolium bistrifluoromethanesulfonimide, [EMIM][Tf $\left.{ }_{2} \mathrm{~N}\right]$

${ }^{1} \mathrm{H}$ NMR in $\mathrm{CD}_{2} \mathrm{Cl}_{2}, \delta: 8.60(\mathrm{~s}, 1 \mathrm{H}, \mathrm{NCHN}) ; 7.35-7.28(\mathrm{~m}, 2 \mathrm{H}, \mathrm{NCHCHN}) ; 4.22(\mathrm{q}, 7.4 \mathrm{~Hz}, 2 \mathrm{H}$, $\left.\mathrm{NCH}_{2} \mathrm{CH}_{3}\right) ; 3.91\left(\mathrm{~s}, 3 \mathrm{H}, \mathrm{NCH}_{3}\right) ; 1.53\left(\mathrm{t}, 7.4 \mathrm{~Hz}, 3 \mathrm{H}, \mathrm{CH}_{2} \mathrm{CH}_{3}\right)$. 
1-propyl-3-methylimidazolium bistrifluoromethanesulfonimide, $\left[\mathrm{C}_{3} \mathrm{MIM}\right]\left[\mathrm{Tf}_{2} \mathrm{~N}\right]$

${ }^{1} \mathrm{H}$ NMR in $\mathrm{CD}_{2} \mathrm{Cl}_{2}, \delta: 8.61(\mathrm{~s}, 1 \mathrm{H}, \mathrm{NCHN}) ; 7.32-7.29(\mathrm{~m}, 2 \mathrm{H}, \mathrm{NCHCHN}) ; 4.13(\mathrm{t}, 7.4 \mathrm{~Hz}, 2 \mathrm{H}$, $\mathrm{NCH}_{2} \mathrm{CH}_{2}$ ); 3.92 (s, $\left.3 \mathrm{H}, \mathrm{NCH}_{3}\right) ; 1.95-1.85\left(\mathrm{~m}, 2 \mathrm{H}, \mathrm{NCH}_{2} \mathrm{CH}_{2}\right) ; 0.96\left(\mathrm{t}, 7.4 \mathrm{~Hz}, 3 \mathrm{H}, \mathrm{CH}_{2} \mathrm{CH}_{3}\right)$.

1-butyl-3-methylimidazolium bistrifluoromethanesulfonimide, [BMIM] [Tf $\left.{ }_{2} \mathrm{~N}\right]$

${ }^{1} \mathrm{H}$ NMR in $\mathrm{CD}_{2} \mathrm{Cl}_{2}, \delta: 8.61(\mathrm{~s}, 1 \mathrm{H}, \mathrm{NCHN}) ; 7.32-7.28(\mathrm{~m}, 2 \mathrm{H}, \mathrm{NCHCHN}) ; 4.15(\mathrm{t}, 7.4 \mathrm{~Hz}, 2 \mathrm{H}$, $\left.\mathrm{NCH}_{2} \mathrm{CH}_{2}\right) ; 3.92\left(\mathrm{~s}, 3 \mathrm{H}, \mathrm{NCH}_{3}\right) ; 1.89-1.80\left(\mathrm{~m}, 2 \mathrm{H}, \mathrm{NCH}_{2} \mathrm{CH}_{2}\right) ; 1.41-1.30\left(\mathrm{~m}, 2 \mathrm{H}, \mathrm{CH}_{2} \mathrm{CH}_{3}\right)$; $0.96\left(\mathrm{t}, 7.4 \mathrm{~Hz}, 3 \mathrm{H}, \mathrm{CH}_{2} \mathrm{CH}_{3}\right)$.

1-pentyl-3-methylimidazolium bistrifluoromethanesulfonimide, $\left[\mathrm{C}_{5} \mathrm{MIM}\right]\left[\mathrm{Tf}_{2} \mathrm{~N}\right]$

${ }^{1} \mathrm{H} \mathrm{NMR}$ in $\mathrm{CD}_{2} \mathrm{Cl}_{2}, \delta: 8.61(\mathrm{~s}, 1 \mathrm{H}, \mathrm{NCHN}) ; 7.31-7.29(\mathrm{~m}, 2 \mathrm{H}, \mathrm{NCHCHN}) ; 4.15(\mathrm{t}, 7.4 \mathrm{~Hz}, 2 \mathrm{H}$, $\left.\mathrm{NCH}_{2} \mathrm{CH}_{2}\right) ; 3.92\left(\mathrm{~s}, 3 \mathrm{H}, \mathrm{NCH}_{3}\right) ; 1.91-1.81\left(\mathrm{~m}, 2 \mathrm{H}, \mathrm{NCH}_{2} \mathrm{CH}_{2}\right) ; 1.42-1.25\left(\mathrm{~m}, 4 \mathrm{H}, \mathrm{CH}_{2} \mathrm{CH}_{2} \mathrm{CH}_{3}\right)$; $0.90\left(\mathrm{t}, 7.0 \mathrm{~Hz}, 3 \mathrm{H}, \mathrm{CH}_{2} \mathrm{CH}_{3}\right)$.

1-butyl-2,3-dimethylimidazolium bistrifluoromethanesulfonimide, [BMMIM][Tf $2 \mathrm{~N}]$

${ }^{1} \mathrm{H} \mathrm{NMR}$ in $\mathrm{CD}_{2} \mathrm{Cl}_{2}, \delta: 7.22-7.18(\mathrm{~m}, 2 \mathrm{H}, \mathrm{NCHCHN}) ; 4.04\left(\mathrm{t}, 7.4 \mathrm{~Hz}, 2 \mathrm{H}, \mathrm{NCH}_{2} \mathrm{CH}_{2}\right) ; 3.78(\mathrm{~s}$, $\left.3 \mathrm{H}, \mathrm{NCH}_{3}\right) ; 2.58\left(\mathrm{~s}, 3 \mathrm{H}, \mathrm{NC}\left(\mathrm{CH}_{3}\right) \mathrm{N}\right) ; 1.82-1.73\left(\mathrm{~m}, 2 \mathrm{H}, \mathrm{NCH}_{2} \mathrm{CH}_{2}\right) ; 1.42-1.31(\mathrm{~m}, 2 \mathrm{H}$, $\left.\mathrm{CH}_{2} \mathrm{CH}_{3}\right) ; 0.96\left(\mathrm{t}, 7.4 \mathrm{~Hz}, 3 \mathrm{H}, \mathrm{CH}_{2} \mathrm{CH}_{3}\right)$.

1-butylpyridinium bistrifluoromethanesulfonimide, $[\mathrm{BPy}]\left[\mathrm{Tf}_{2} \mathrm{~N}\right]$

${ }^{1} \mathrm{H}$ NMR in $\mathrm{CD}_{2} \mathrm{Cl}_{2}, \delta: 8.74(\mathrm{~d}, 5.4 \mathrm{~Hz}, 2 \mathrm{H}, \mathrm{NCHCH}) ; 8.54-8.47(\mathrm{~m}, 2 \mathrm{H}, \mathrm{NCHCHCH}) ; 8.07(\mathrm{t}$, 7.0 Hz, $1 \mathrm{H}, \mathrm{NCHCHCH}) ; 4.58\left(\mathrm{t}, 7.6 \mathrm{~Hz}, 2 \mathrm{H}, \mathrm{NCH}_{2} \mathrm{CH}_{2}\right) ; 2.05-1.94\left(\mathrm{~m}, 2 \mathrm{H} . \mathrm{NCH}_{2} \mathrm{CH}_{2}\right) ; 1.46-$ $1.35\left(\mathrm{~m}, 2 \mathrm{H}, \mathrm{CH}_{2} \mathrm{CH}_{2} \mathrm{CH}_{3}\right) ; 0.98\left(\mathrm{t}, 7.4 \mathrm{~Hz}, 3 \mathrm{H}, \mathrm{CH}_{2} \mathrm{CH}_{3}\right)$. 


\section{S-11}

1-butyl-1-methylpyrrolidinium bistrifluoromethanesulfonimide, [BMPyr][ $\left.\mathrm{Tf}_{2} \mathrm{~N}\right]$

${ }^{1} \mathrm{H}$ NMR in $\mathrm{CD}_{2} \mathrm{Cl}_{2}, \delta: 3.54-3.41\left(\mathrm{~m}, 4 \mathrm{H}, \mathrm{NCH}_{2} \mathrm{CH}_{2} \mathrm{CH}_{2} \mathrm{CH}_{2} \mathrm{~N}\right) ; 3.31-3.24\left(\mathrm{~m}, 2 \mathrm{H}, \mathrm{NCH}_{2} \mathrm{CH}_{2}\right)$; 3.02 (s, 3H, NCH $) ; 2.33-2.19$ (m, 4H, $\left.\mathrm{NCH}_{2} \mathrm{CH}_{2} \mathrm{CH}_{2} \mathrm{CH}_{2} \mathrm{~N}\right) ; 1.80-1.69$ (m, 2H, $\mathrm{NCH}_{2} \mathrm{CH}_{2}$ ); 1.48-1.36 (m, 2H, $\left.\mathrm{CH}_{2} \mathrm{CH}_{3}\right) ; 0.99$ (t, $\left.7.4 \mathrm{~Hz}, 3 \mathrm{H}, \mathrm{CH}_{2} \mathrm{CH}_{3}\right)$.

1-pentyl-1-methylpyrrolidinium bistrifluoromethanesulfonimide, [ $\left.\mathrm{C}_{5} \mathrm{MPyr}\right]\left[\mathrm{Tf}_{2} \mathrm{~N}\right]$

${ }^{1} \mathrm{H}$ NMR in $\mathrm{CD}_{2} \mathrm{Cl}_{2}, \delta: 3.54-3.41\left(\mathrm{~m}, 4 \mathrm{H}, \mathrm{NCH}_{2} \mathrm{CH}_{2} \mathrm{CH}_{2} \mathrm{CH}_{2} \mathrm{~N}\right) ; 3.30-3.23\left(\mathrm{~m}, 2 \mathrm{H}, \mathrm{NCH}_{2} \mathrm{CH}_{2}\right)$; 3.02 (s, 3H, $\left.\mathrm{NCH}_{3}\right) ; 2.32-2.19$ (m, 4H, $\left.\mathrm{NCH}_{2} \mathrm{CH}_{2} \mathrm{CH}_{2} \mathrm{CH}_{2} \mathrm{~N}\right) ; 1.82-1.70\left(\mathrm{~m}, 2 \mathrm{H}, \mathrm{NCH}_{2} \mathrm{CH}_{2}\right.$ ); 1.46-1.30 (m, 4H, $\left.\mathrm{CH}_{2} \mathrm{CH}_{2} \mathrm{CH}_{3}\right) ; 0.93\left(\mathrm{t}, 7.0 \mathrm{~Hz}, 3 \mathrm{H}, \mathrm{CH}_{2} \mathrm{CH}_{3}\right)$.

1-pentyl-1,1,1-triethylammonium bistrifluoromethanesulfonimide, $\left[\mathrm{C}_{5} \mathrm{NEt}_{3}\right]\left[\mathrm{Tf}_{2} \mathrm{~N}\right]$

${ }^{1} \mathrm{H}$ NMR in $\mathrm{CD}_{2} \mathrm{Cl}_{2}, \delta: 3.22\left(\mathrm{q}, 7.4 \mathrm{~Hz}, 6 \mathrm{H}, \mathrm{NCH}_{2} \mathrm{CH}_{3}\right) ; 3.08-3.01\left(\mathrm{~m}, 2 \mathrm{H}, \mathrm{NCH}_{2} \mathrm{CH}_{2}\right) ; 1.67-1.57$ (m, 2H, $\left.\mathrm{NCH}_{2} \mathrm{CH}_{2}\right) ; 1.45-1.27$ (m, 4H, $\left.\mathrm{CH}_{2} \mathrm{CH}_{2} \mathrm{CH}_{3}\right) ; 1.24$ (t, $\left.7.4 \mathrm{~Hz}, 9 \mathrm{H}, \mathrm{NCH}_{2} \mathrm{CH}_{3}\right) ; 0.93$ (t, $7.0 \mathrm{~Hz}, 3 \mathrm{H}, \mathrm{CH}_{2} \mathrm{CH}_{2} \mathrm{CH}_{3}$ ).

1-butyl-3-methylimidazolium hexafluorophosphate, [BMIM][PF 6$]$

${ }^{1} \mathrm{H}$ NMR in $\mathrm{CD}_{2} \mathrm{Cl}_{2}, \delta: 8.43(\mathrm{~s}, 1 \mathrm{H}, \mathrm{NCHN}) ; 7.31-7.29(\mathrm{~m}, 2 \mathrm{H}, \mathrm{NCHCHN}) ; 4.14(\mathrm{t}, 7.4 \mathrm{~Hz}, 2 \mathrm{H}$, $\left.\mathrm{NCH}_{2} \mathrm{CH}_{2}\right) ; 3.89\left(\mathrm{~s}, 3 \mathrm{H}, \mathrm{NCH}_{3}\right) ; 1.89-1.79\left(\mathrm{~m}, 2 \mathrm{H}, \mathrm{NCH}_{2} \mathrm{CH}_{2}\right) ; 1.41-1.29\left(\mathrm{~m}, 2 \mathrm{H}, \mathrm{CH}_{2} \mathrm{CH}_{3}\right)$; $0.94\left(\mathrm{t}, 7.4 \mathrm{~Hz}, 3 \mathrm{H}, \mathrm{CH}_{2} \mathrm{CH}_{3}\right)$.

1-butyl-3-methylimidazolium trifluoromethanesulfonate, [BMIM][TfO]

${ }^{1} \mathrm{H}$ NMR in $\mathrm{CD}_{2} \mathrm{Cl}_{2}, \delta: 9.02(\mathrm{~s}, 1 \mathrm{H}, \mathrm{NCHN}) ; 7.35-7.31(\mathrm{~m}, 2 \mathrm{H}, \mathrm{NCHCHN}) ; 4.18(\mathrm{t}, 7.4 \mathrm{~Hz}, 2 \mathrm{H}$, $\left.\mathrm{NCH}_{2} \mathrm{CH}_{2}\right) ; 3.94\left(\mathrm{~s}, 3 \mathrm{H}, \mathrm{NCH}_{3}\right) ; 1.90-1.81\left(\mathrm{~m}, 2 \mathrm{H}, \mathrm{NCH}_{2} \mathrm{CH}_{2}\right) ; 1.41-1.30\left(\mathrm{~m}, 2 \mathrm{H}, \mathrm{CH}_{2} \mathrm{CH}_{3}\right)$; $0.95\left(\mathrm{t}, 7.4 \mathrm{~Hz}, 3 \mathrm{H}, \mathrm{CH}_{2} \mathrm{CH}_{3}\right)$. 
1-butyl-3-methylimidazolium tetrafluoroborate, [BMIM] $\left[\mathrm{BF}_{4}\right]$

${ }^{1} \mathrm{H}$ NMR in $\mathrm{CD}_{2} \mathrm{Cl}_{2}, \delta: 8.71(\mathrm{~s}, 1 \mathrm{H}, \mathrm{NCHN}) ; 7.37-7.32(\mathrm{~m}, 2 \mathrm{H}, \mathrm{NCHCHN}) ; 4.16(\mathrm{t}, 7.4 \mathrm{~Hz}, 2 \mathrm{H}$, $\left.\mathrm{NCH}_{2} \mathrm{CH}_{2}\right) ; 3.92\left(\mathrm{~s}, 3 \mathrm{H}, \mathrm{NCH}_{3}\right) ; 1.89-1.79\left(\mathrm{~m}, 2 \mathrm{H}, \mathrm{NCH}_{2} \mathrm{CH}_{2}\right) ; 1.40-1.29\left(\mathrm{~m}, 2 \mathrm{H}, \mathrm{CH}_{2} \mathrm{CH}_{3}\right)$; $0.94\left(\mathrm{t}, 7.4 \mathrm{~Hz}, 3 \mathrm{H}, \mathrm{CH}_{2} \mathrm{CH}_{3}\right)$.

Melting points were obtained by differential scanning calorimetry (DSC), performed on a Setaram DSC131 instrument. The samples were placed into aluminium pans and sealed in a dry box. The DSC data were recorded at a scanning rate of $5^{\circ} \mathrm{C} / \mathrm{min}$ for cooling and heating. The estimated error for melting point determinations is $\pm 0.3{ }^{\circ} \mathrm{C}$.

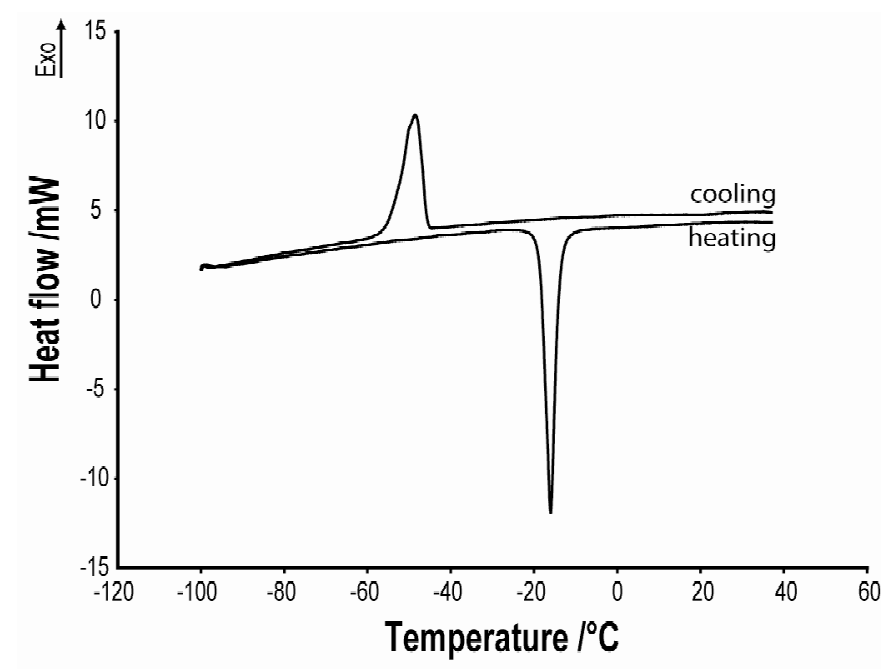

Figure S1 : DSC plot for [EMIM] $\left[\mathrm{Tf}_{2} \mathrm{~N}\right]$ 


\section{S-13}

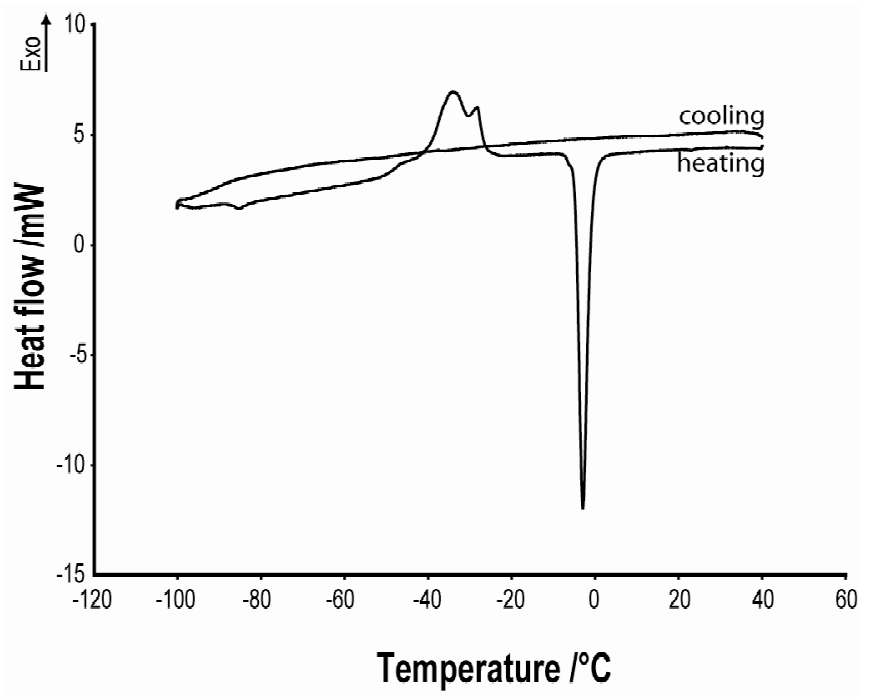

Figure S2 : DSC plot for [BMIM] $\left[\mathrm{Tf}_{2} \mathrm{~N}\right]$

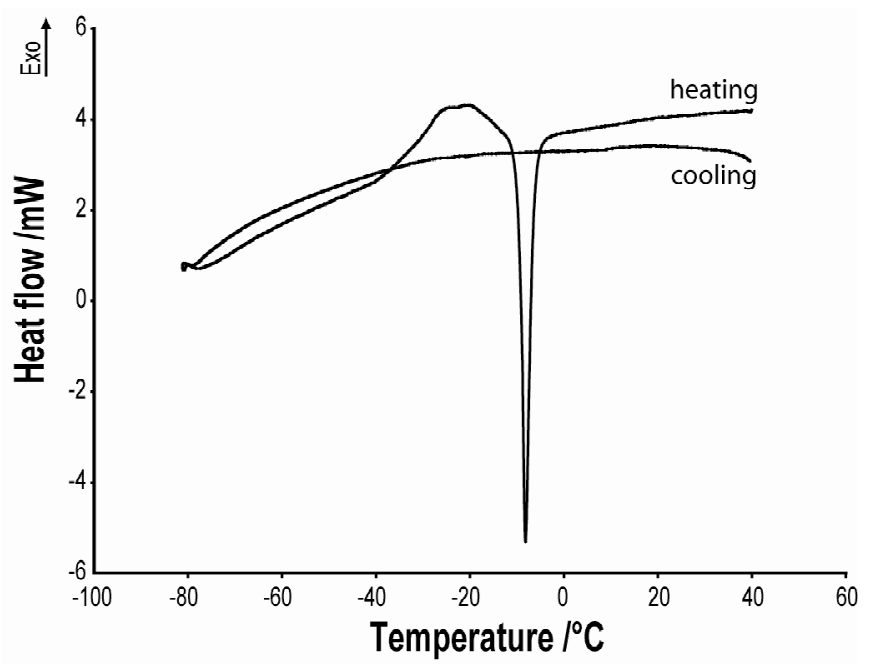

Figure S3 : DSC plot for $\left[\mathrm{C}_{5} \mathrm{MIM}\right]\left[\mathrm{Tf}_{2} \mathrm{~N}\right]$ 


\section{S-14}

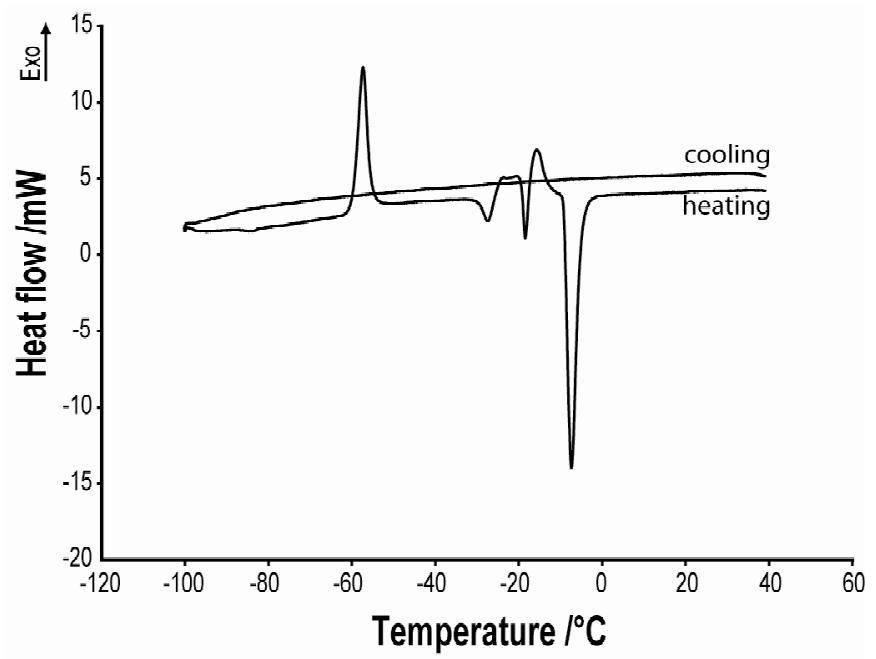

Figure S4 : DSC plot for [BMPy] $\left[\mathrm{Tf}_{2} \mathrm{~N}\right]$

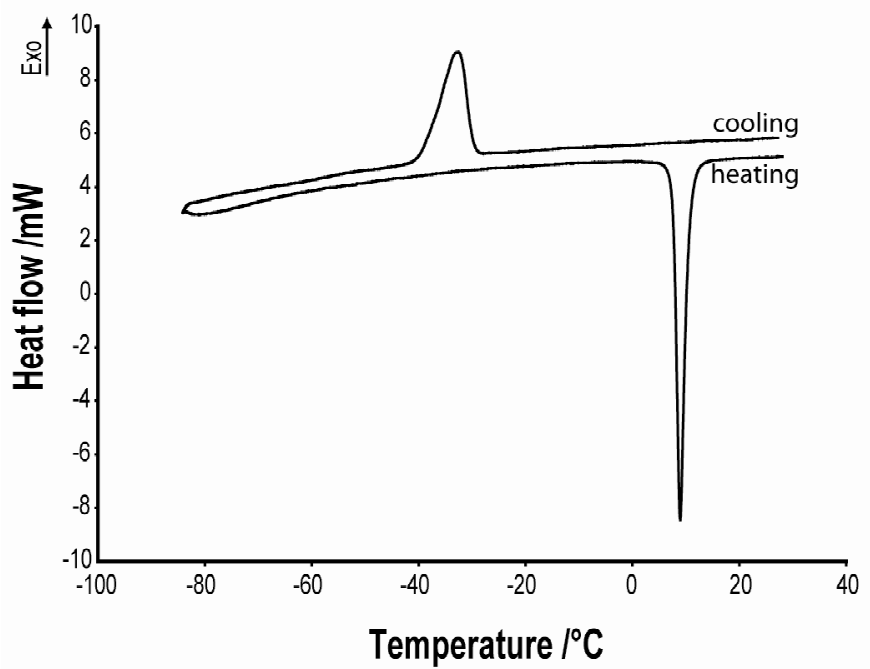

Figure S5 : DSC plot for $\left[\mathrm{C}_{5} \mathrm{MPy}\right]\left[\mathrm{Tf}_{2} \mathrm{~N}\right]$ 
S-15

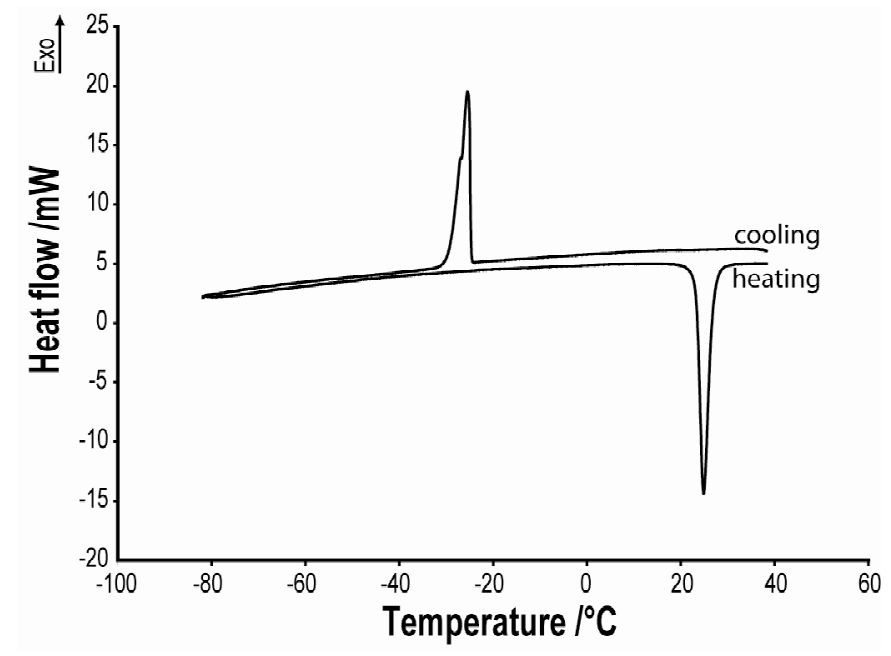

Figure S6 : DSC plot for [BPy] $\left[\mathrm{Tf}_{2} \mathrm{~N}\right]$

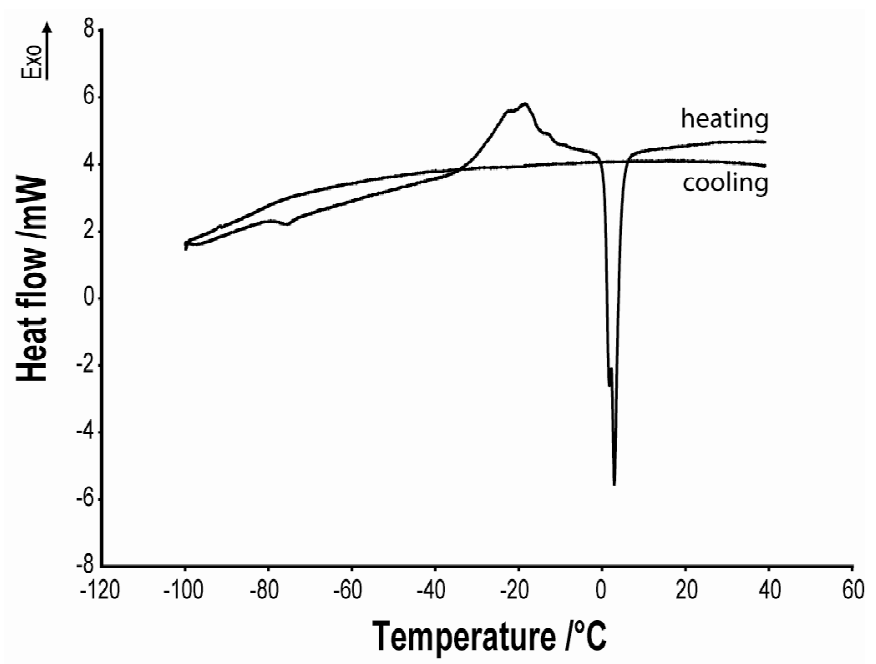

Figure S7 : DSC plot for $\left[\mathrm{C}_{5} \mathrm{NEt}_{3}\right]\left[\mathrm{Tf}_{2} \mathrm{~N}\right]$ 


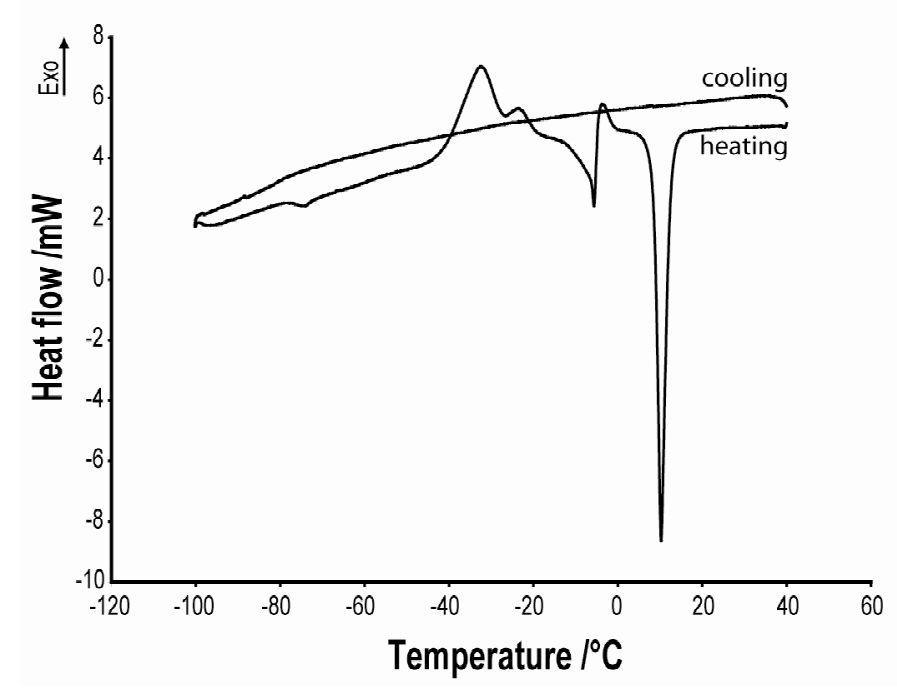

Figure S10 : DSC plot for $[\mathrm{BMIM}]\left[\mathrm{PF}_{6}\right]$

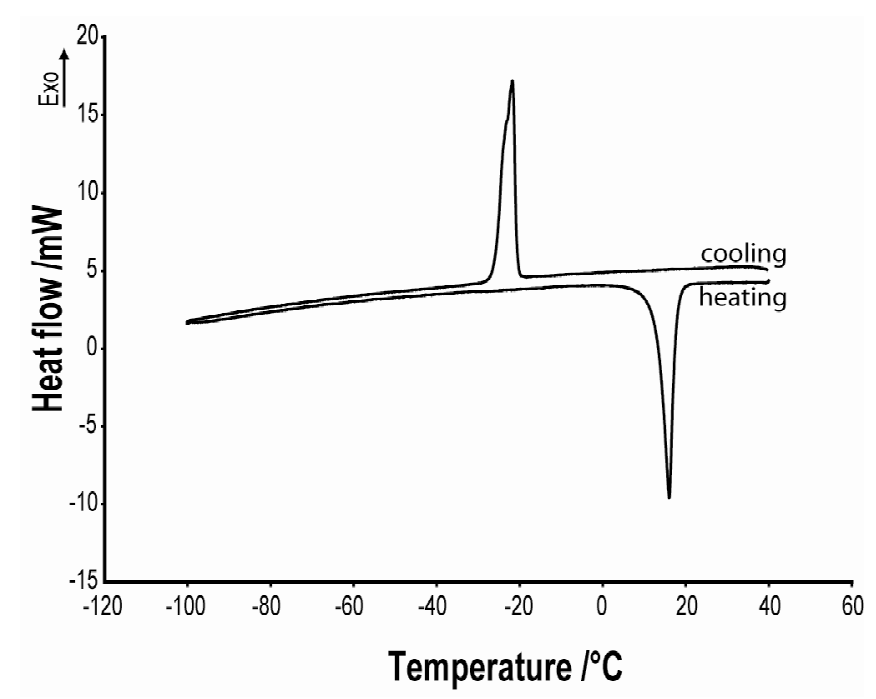

Figure S11 : DSC plot for [BMIM][TfO]

Dielectric constants for all salts were determined at $25{ }^{\circ} \mathrm{C}$ using previously reported techniques. ${ }^{\text {(vii, viii) }}$ During the present study, the dielectric constants for [EMIM][TfO] and $[\mathrm{EMIM}]\left[\mathrm{BF}_{4}\right]$ were re-evaluated using old data. The values obtained were very similar, but the re-evaluated data are presented herein. The estimated error for dielectric constant determinations during this study is \pm 0.3 . 


\section{S-17}

Dielectric constants were also measured over a range of temperatures $\left(15,25,3545{ }^{\circ} \mathrm{C}\right)$ for the salts $[\mathrm{EMIM}]\left[\mathrm{Tf}_{2} \mathrm{~N}\right]$ and $[\mathrm{BMIM}]\left[\mathrm{Tf}_{2} \mathrm{~N}\right]$. These measurements required the use of a different calibration standard, benzonitrile rather than methanol (as used for the $25{ }^{\circ} \mathrm{C}$ measurements). The most accurately known standard which proved to be suitable in a long array of experiments at $298 \mathrm{~K}$ is methanol, and the measurements at $25{ }^{\circ} \mathrm{C}$ have been conducted with methanol. This should give the best values. However, above $25{ }^{\circ} \mathrm{C}$ accurate methanol data are lacking and the only way out was to use benzonitrile as the calibration standard, where accurate calibration data are available in the temperature range of these experiments. The change of calibration standard led to a systematic difference in the dielectric constant of +0.3 compared to the values determined using a methanol standard. For internal consistency of the two data sets, the data presented in the text were corrected for this effect.

The dielectric constant of a mixture of [BMIM][TfO] and $1 \%$ by weight of water was also measured to determine the effect of water impurities on the static dielectric constant measured using dielectric spectroscopy. The $\mathrm{IL} / \mathrm{H}_{2} \mathrm{O}$ mixture was found to have a dielectric constant of 13.9 compared to the value of 13.2 for the dry salt. The relatively small effect of this water content on the dielectric constant of the IL suggests that if traces of water are present in the ILs tested above, this will have a negligible effect on the dielectric constant measured using this technique. 


\title{
Calculation Details:
}

\section{XYZ Coordinates and SCF Energies (AU)}

\author{
Calculated using TURBOMOLE \\ (RI)-BP86/SV(P) level
}

Note: lower case letters after the atomic coordinates indicate the atomic symbol of the atoms involved in the calculations.

\begin{tabular}{|c|c|c|}
\hline Anions: & & \\
\hline$\left[\mathrm{BF}_{4}\right]^{-}$ & & \\
\hline SCF Energy $=-424.20$ & 9937748 & \\
\hline $\mathrm{X}$ & $\mathrm{y}$ & $\mathrm{Z}$ \\
\hline 0.00000000000000 & 0.00000000000000 & 0.00000000000000 \\
\hline 1.54621771810347 & 1.54621771810347 & 1.54621771810347 \\
\hline-1.54621771810347 & 1.54621771810347 & -1.54621771810347 \\
\hline-1.54621771810347 & -1.54621771810347 & 1.54621771810347 \\
\hline 1.54621771810347 & -1.54621771810347 & -1.54621771810347 \\
\hline
\end{tabular}

$\begin{array}{lrrr}\left.\mathbf{P F}_{\mathbf{6}}\right]^{-} & & & \\ \text {SCF Energy }=-940.1539310097 & & \\ 0.00000000000000 & 0.00000000000000 & 0.00000000000000 & \mathrm{p} \\ 3.12416034495795 & 0.00000000000000 & 0.00000000000000 & \mathrm{f} \\ 0.00000000000000 & 3.12416034495795 & 0.00000000000000 & \mathrm{f} \\ -3.12416034495795 & 0.00000000000000 & 0.00000000000000 & \mathrm{f} \\ 0.00000000000000 & -3.12416034495795 & 0.00000000000000 & \mathrm{f} \\ 0.00000000000000 & 0.00000000000000 & 3.12416034825047 & \mathrm{f} \\ 0.00000000000000 & 0.00000000000000 & -3.12416034825047 & \mathrm{f}\end{array}$

\section{[TfO]}

SCF Energy = -961.0428040008

1.75420226849609

2.72566679475866

2.72497822992111

2.71867286896198

$-1.84867662680278$

$-2.44021436030705$

$-2.43464562483473$

$-2.43778850705203$
$-0.00039065303771$

2.20706382652819

$-1.87580356647675$

$-0.33302231153244$

0.00027480195209

$-2.55939641092011$

0.38197448183088

2.17925681672870
$-0.00010756979929$

$-0.88916394503019$

$-1.46511976773086$

2.35944963446427

$-0.00153861974428$

1.03718848787932

$-2.73816061211261$

1.69800214370833

\section{$\left[\mathrm{Tf}_{2} \mathbf{N}\right]^{-}$}

SCF Energy = -1826.372074047

$-0.24470505021272$

1.99569309465050

$-1.76822839558403$

0.47668897572644

1.57763858364577

0.54060635687588

0.04326748296443

$-2.17553524589908$

$-2.87761530852476$

$-2.00199198229046$

$2.05725771174247 \quad \mathrm{~s}$

$-1.47762879143445 \quad$ o 
S-19

-3.34052537432467
0.77628840371808
2.74814459159338
-0.25727332619209
1.64488742566048
2.65944041369286
-0.62777177107312
0.77000518624985
-2.03621568692925
-2.19175615320632
3.23854839880667

$-3.34052537432467$

2.36145544563181 $-0.36763804867534$

1.23742764242652

$-0.21309943925231$

$-2.72809349227490$

$-1.90413767546540$

$-0.36957296811591$

$-1.13286746690051$

1.61850347324224

$-2.26344100348911$

2.44641959114986
$-3.51655251826722 \quad$ o

$-4.63472669265889 \quad \mathrm{c}$

$-4.54570598143015 \mathrm{f}$

$-6.96898648535630 \mathrm{f}$

$-4.32236610759000 \mathrm{f}$

$1.20181200600267 \quad$ o

$4.75537483070734 \quad \mathrm{c}$

$6.75853769737497 \mathrm{f}$

$5.50087919140676 \mathrm{f}$

$4.12224619515480 \mathrm{f}$

$3.25426656258448 \quad$ o

\section{Cations:}

\section{[EMIM] $^{+}$}

SCF Energy = -344.2905963481

$-2.27787708682348$

0.26622510966512

1.58852555222213

$-0.19992271535640$

$-0.07565401540524$

$-0.03152770874205$

0.39639624324485

0.58494555079220

$-2.46332438409244$

0.21283465607238

1.33955930298572

$-0.44218941443613$

0.67746690304667

0.51777837832244

1.03077984835885

$-1.36030509994065$

1.07157772701290

$-0.88738305841909$

$-3.74168488092515$

$-0.55225842193687$

3.64745728002300

0.60548510090529

$-4.12323126945594$

0.29091133430584

3.33770795915122

0.17662626916645

1.23374337743315

$-2.46751903855258$

0.26587940804018

0.71067470026812

2.61971494467562

$-0.59558225192291$

1.81826826720168

2.53650232159236

$-1.43214238945213$

$-2.13640253935464$

1.81788175373955

$-2.85349411118813$

$2.23343947241819 \quad \mathrm{c}$

$2.85186361200266 \mathrm{n}$

$0.72442272831632 \quad \mathrm{c}$

$-1.23124864155541 \mathrm{n}$

$-0.32890303590252 \quad \mathrm{c}$

$5.38612891315209 \quad \mathrm{c}$

$-3.90785791175249 \quad \mathrm{c}$

$-5.52350275209332 \mathrm{c}$

$-7.49224478375799 \quad \mathrm{~h}$

$3.65646582443316 \mathrm{~h}$

$0.60706790050283 \mathrm{~h}$

$-1.56548274213618 \quad \mathrm{~h}$

$5.38275667926139 \quad \mathrm{~h}$

$5.91949850852615 \mathrm{~h}$

$6.76624683818973 \mathrm{~h}$

$-3.88722712934799 \quad \mathrm{~h}$

$-4.62489866089334 \quad h$

$-5.58017971176108 \quad \mathrm{~h}$

$-4.82542984573894 \quad h$

\section{[C $\left.\mathrm{C}_{3} \mathrm{MIM}\right]^{+}$}

SCF Energy $=-383.5728594674$

$-2.17278658073911-0.36475694697623$

0.40336323591596

$-0.11547288730951$

3.35347254059796

$\mathrm{c}$

$3.76964850115682 \mathrm{n}$

$1.56541357198604 \quad \mathrm{c}$

1.51496380219106

0.52107908861783

$-0.27041234161457$

0.69811902043315

$-2.59290045992231$

0.14187537809332

$-0.24071870633339 \mathrm{n}$

1.70284230606039

$-0.52523635308368$

0.83595687642810

c

0.19261831735427

1.26951683479095

$6.18867770728804 \quad \mathrm{c}$

0.14251912355463

$-1.09765877566986$

0.65543860805525

$-0.44242078531191$

$-2.94121950575165 \quad \mathrm{c}$

$-4.62267705093147 \quad \mathrm{c}$

0.59613916609583

$-2.17761094119370$

$-7.40090082733423$

$-8.57818121454016$

c

$-3.49005869133943$

$-0.86316595466152$

4.87269796529203

$\mathrm{h}$ 
S-20

3.54171486606820
-4.35032321000749
3.64727824647288
1.77694588244203
0.67070791488918
2.05028909765710
-1.26694476381337
-1.73011512905532
1.57790940022847
2.54974149619628
-0.77981893501173

3.54171486606820

$-4.35032321000749$

1.77694588244203

0.67070791488918

2.05028909765710

$-1.26694476381337$

1.57790940022847

$-0.77981893501173$
0.85537085763750

0.16987358480215

0.23429682303730

$-2.57591917895306$

0.48148672260972

2.23643564218310

2.65743995626516

$-2.04007892227802$

$-2.46713079137361$

0.43173854378761

0.89035132844593
$1.29077962465809 \quad \mathrm{~h}$

$-0.25921524630343 \quad h$

$6.06132368608753 \mathrm{~h}$

$6.62134925971876 \mathrm{~h}$

$7.70777457710255 \quad \mathrm{~h}$

$-3.04573837282061 \mathrm{~h}$

$-3.52964923649911 \mathrm{~h}$

$-4.43839749554303 \mathrm{~h}$

$-3.92080362638767 \quad \mathrm{~h}$

$-7.65486224887853 \quad h$

$-8.16283614632397 \quad h$

\section{[BMIM] $^{+}$}

SCF Energy $=-422.8544286918$

$-2.03500936642739$

0.49997959094673

1.41927960688212

$-0.45023474709617$

$-2.62788265004603$

1.94461615109852

$-0.18963042321989$

0.09087870295208

0.30282571373403

0.60957123618910

$-3.20645711626001$

3.36908396789539

$-4.41850171687851$

3.79023480344600

2.25517056956196

0.88334009759717

1.47352205846330

$-1.88229314283020$

$-1.56323302438456$

1.79468696613426

1.93690972088561

$-1.40933493829151$

0.74127245005726

$-1.02468547226947$

2.35256223122147
$-0.81528912189608$

$-0.25812792878401$

0.75626830942536

0.87020739492321

$-0.11032140883633$

$-0.73481388779605$

1.77322929478211

$-0.39158356917375$

0.59232352215281

$-1.54243329637271$

$-1.64763546545878$

1.39507939077591

$-0.20924288598608$

0.24070074460710

$-2.79557700758813$

0.01322963660147

3.05015481039273

2.94340056822088

$-1.68489791556808$

$-1.52875129337949$

1.91455125122324

1.72890241538963

$-0.76670238144713$

$-2.86459354610915$

$-2.66221758657067$
4.31290861523155

4.67977888255619

2.52843139357181

0.80369308194416

1.87947127286845

7.00227862196835

$-1.83377456378236$

$-3.74585890334923$

$-6.47533953531134$

$-8.41307649351100$

5.80483498995712

2.23356267441169

0.84297065077894

6.87533322621711

7.23719310970714

8.64600677365229

$-1.87726569985507$

$-2.24214289597469$

$-3.58362311545464$

$-3.25721599042116$

$-6.61462040663571$

$-6.93767270180761$

$-10.35882058429706$

$-8.36814869859855$

$-8.05665625816323$ c

n

c

$\mathrm{n}$

c

$\mathrm{c}$

c

c

c

c

$\mathrm{h}$

$\mathrm{h}$

$\mathrm{h}$

$\mathrm{h}$

$\mathrm{h}$

$\mathrm{h}$

$\mathrm{h}$

$\mathrm{h}$

$\mathrm{h}$

h

$\mathrm{h}$

h

$\mathrm{h}$

$\mathrm{h}$

$\mathrm{h}$

\section{[C $_{5}$ MIM] ${ }^{+}$}

SCF Energy = -462.1358128574

$-1.96003780871887$

0.57564412173234

1.42510806586386

$-0.87921835040743$

$-0.29225675149784$

5.39350067759770

c

0.76559751227834

5.70326770149835

3.54443650845481

1.87039536395723

0.88038049103575

$-0.48976820204999$

$-0.14607834005825$

$-2.62557913621351$

2.08486549539472

$-0.77525268256646$

2.98715875662741

7.98263457707601

1.82448946252085

$-0.76050383601526$ 


-0.07730251316407
0.10352794329078
0.32094205556727
-3.08062431590371
3.35738329770229
-4.44079184048733
3.94234367593755
2.36795472813533
1.09138997843750
1.35065077326510
-2.01482045704322
-1.74177863056755
1.62176021515580
1.76973581554238
-1.58789617476082
-1.34779100900561
2.00516789910903
0.51696181862033
-1.17651532100496
0.67856939692834
2.20493811453829

$-0.30967209211191$

0.72071302349200

$-1.37902097778184$

$-1.75078547182667$

1.43692295571538

$-0.25664735971720$

0.16580592731874

$-2.83880067658739$

0.00497835973661

3.10609105964916

2.99563025863263

$-1.58895301737254$

$-1.47137912850931$

2.00358043995865

1.90868186790243

$-2.65591295192172$

$-2.56985432853344$

$-0.35739210151099$

0.77545425319937

$-1.92610112021483$

0.87385113814385
$-2.71312034765872 \quad \mathrm{c}$

$-5.42590556065720 \quad \mathrm{c}$

$-7.42615675743798 \quad \mathrm{c}$

$6.90192982918297 \mathrm{~h}$

$3.20992875129062 \mathrm{~h}$

$1.99562460186862 \quad \mathrm{~h}$

$7.78770331588340 \quad \mathrm{~h}$

$8.22475313807171 \quad \mathrm{~h}$

$9.65381455962647 \quad \mathrm{~h}$

$-0.83118120094186 \mathrm{~h}$

$-1.10253814617005 \quad \mathrm{~h}$

$-2.54673886612117 \mathrm{~h}$

$-2.26997578804452 \quad h$

$-5.57834129636982 \mathrm{~h}$

$-5.83916135449846 \quad \mathrm{~h}$

$-7.27009883824089 \quad h$

$-6.99518113066095 \mathrm{~h}$

$-10.13151985933193 \quad c$

$-10.65186318100737 \mathrm{~h}$

$-11.51809407565752 \mathrm{~h}$

$-10.36909784070074 \mathrm{~h}$

\section{[BMMIM] $^{+}$}

SCF Energy $=-462.1472549943$

$-3.45367158877374$

$-4.32671050450138$

$-2.31876798365620$

$-0.19186161697135$

$-0.86253077641638$

$-7.00053023970026$

2.44036933553716

3.59774235795724

6.32641168263737

7.55243422337630

$-2.44340639197535$

$-4.74068357147842$

0.55273251180578

$-7.38971086919914$

$-8.16285050393157$

$-7.51437828499918$

2.43587655416191

3.54529792133322

3.56565124120715

2.40365894764246

6.33613457884969

7.48204031314165

9.50353352796710

7.67280556224113

6.46662893016746
3.49005695705850

2.13827675788860

1.23318892153461

1.99130594313290

3.39355398868053

1.81783143891598

1.34537250303945

$-0.68034435533810$

$-1.33204930607649$

$-3.27963527425830$

$-0.31820832562190$

4.40995042611741

4.21014117517480

$-0.17746162373010$

2.32453856532074

3.07228803260763

0.71296657979020

3.12761654560982

0.00415110260652

$-2.41383895126128$

$-2.05947652223535$

0.42891311768665

$-3.72998518026016$

$-2.56273098556593$

$-5.07965701376163$
$-1.62623583613228 \quad \mathrm{c}$

$0.44415661548105 \mathrm{n}$

$1.77115326723884 \quad \mathrm{c}$

$0.54909876973892 \mathrm{n}$

$-1.56124187831288 \quad \mathrm{c}$

$1.11323180650684 \quad \mathrm{c}$

$1.23049271332257 \quad \mathrm{c}$

$-0.49798045912234 \quad \mathrm{c}$

$0.26940173706854 \quad \mathrm{c}$

$-1.49377248472486 \quad \mathrm{c}$

$4.11961674151836 \quad \mathrm{c}$

$-2.96245009293236 \mathrm{~h}$

$-2.83267547408950 \mathrm{~h}$

$1.61324678085926 \mathrm{~h}$

$-0.55007497708633 \mathrm{~h}$

$2.71476603855649 \quad \mathrm{~h}$

$3.22729127501374 \quad \mathrm{~h}$

$1.14994844470822 \quad \mathrm{~h}$

$-2.48946835968545 \quad \mathrm{~h}$

$-0.44228888849910 \mathrm{~h}$

$2.24658610851650 \mathrm{~h}$

$0.29050216705360 \mathrm{~h}$

$-0.86435736773581 \quad \mathrm{~h}$

$-3.46634821420064 \mathrm{~h}$

$-1.52890375374141 \quad h$ 
$-1.05553788273621$

$-2.03820157394960$

$-4.34193690000928$
0.34959324684578

$-2.34571987909211$

$-0.19862159633502$
5.54504480831288

3.72697598540276

4.99317242581042 $\mathrm{h}$

$\mathrm{h}$

$\mathrm{h}$

\section{[BPy $^{+}$}

SCF Energy = -405.6068797877

$-1.13614123693350$

0.87220023805512

0.00513147341107

0.41817456339258

$-2.32167685600791$

$-4.77950871432056$

$-0.53191501533606$

$-6.03968838600028$

$-4.78637047812257$

$-1.02314925436611$

$-2.23347116545291$

$-2.29358652925276 \quad \mathrm{c}$

$-0.00726788397090 \quad \mathrm{c}$

$-0.54471050720023$

$2.28551953530215 \quad \mathrm{c}$

0.40577889857065

1.53835283908885

1.78757851140358

3.45172150816651

$-0.39404410013223$

6.19379451915595

8.14115251547142

$-1.23548857015043$

$-5.69083553048780$

$-7.98423162113056$

$-5.70311469531219$

$-1.24728967612129$

1.76132765978900

1.76188432518780

3.12426026668299

3.12275860571128

6.49278443710875

6.49369945018342

10.09275812485862

7.94246379592344

0.58276658946847

$-1.56551776715976$

0.84475698885369

$-0.87049168773539$

$-1.76750676346372$

$-0.89350125318909$

0.82255633770433

2.99503277305320

3.01605000145816

$-1.59738029593107$

$-1.61911467061878$

1.80058764581980

1.82360341209414

$-0.79331335590358$

$-2.77490530597895$

7.94122048926839

$-2.79847629295273$

$2.23794249379150 \quad \mathrm{c}$

$0.01127000933910 \quad \mathrm{c}$

$-0.00206535400452 \quad \mathrm{c}$

$0.00531064238885 \quad \mathrm{c}$

$-0.00893469923039 \quad \mathrm{c}$

$-3.95609259682398 \mathrm{~h}$

$-4.13256650427558 \quad \mathrm{~h}$

$-0.01218452344166 \quad \mathrm{~h}$

$4.11991012778238 \quad \mathrm{~h}$

$3.96516413294752 \quad \mathrm{~h}$

$1.71008645095567 \mathrm{~h}$

$-1.67239389744725 \quad \mathrm{~h}$

$-1.69918570662593 \mathrm{~h}$

$1.67910388997479 \quad \mathrm{~h}$

$1.69728273724380 \mathrm{~h}$

$-1.66970809358181 \quad \mathrm{~h}$

$-0.00282423604054 \mathrm{~h}$

$-1.71656038991457 \quad \mathrm{~h}$

$1.68161450084736 \mathrm{~h}$

\section{[BMPyr $]^{+}$}

SCF Energy $=-409.1942107836$

5.95487034971183

$-1.37974720967391$

$-3.44355031203735$

$-2.13386340298564$

$-5.89607889875897$

8.25801895406230

1.22638201440415

3.46344918193672

$-5.03769862014119$

$-1.51923468232578$

6.22624092938944

5.80126056519716

$-3.46512728666028$

$-2.87324513612478$

$-1.17329684279605$

$-1.42386423924303$
$-0.95837504018267$

0.25454733490836

$-1.15365772266087$

$-0.24420810121436$

$-0.72362314714936$

0.15006140484835

$-0.80267297884052$

0.36632824516890

$-0.12013110118275$

3.03627229529451

$-0.80577287502347$

$-3.01580287405364$

$-0.46100493512274$

$-3.17017185151944$

1.13638848179834

$-2.16270976492463$
0.48926619256726

0.49644395417421

1.95697007188002

$-2.24847744915323$

0.46133923388055

$-0.87882636523036$

1.15362483171536

$-0.25290142475259$

$-2.29181556761432$

1.08089634189676

2.57256074947865

0.06575616711049

3.93292162654683

1.96466313649725

$-3.49216692791756$

$-2.70349581155688$ c

n

c

c

c

c

c

c

$c$

c

$\mathrm{h}$

$\mathrm{h}$

$\mathrm{h}$

$\mathrm{h}$

h

h 
-7.02540536439032
-7.10009423043359
8.08374820577714
10.01338941315305
8.50818807478953
1.11154314938597
1.43036335389482
3.63532779661825
3.18997127552371
-5.78649157428735
-5.71114652182258
-3.47202228973046
-0.20834999986905
-0.98250961058724
0.84601967363574 $-2.43462362909866$ $-0.04612993804318$ $-0.84072591736173$ 2.19095911080607 $-2.86283585700393$ $-0.56007167526976$ 2.41604763180399 0.19634563612884 $-1.49700441167759$ 1.76793221119437 3.71561459578035 4.07783406263028 3.33488883225179
1.27128469133105

0.56518423541222

$-2.96480190197475$

$-0.29461870109498 \quad \mathrm{~h}$

$-0.44085841046301 \quad h$

$0.76433714239350 \mathrm{~h}$

$3.22970466878660 \quad \mathrm{~h}$

$0.18517286407929 \quad \mathrm{~h}$

$-2.33334057340717 \quad \mathrm{~h}$

$-3.68212691682476 \quad \mathrm{~h}$

$-2.90329274466496 \mathrm{~h}$

$0.76695786766565 \mathrm{~h}$

$-0.17058802256607 \quad \mathrm{~h}$

$3.08285720066076 \mathrm{~h}$

\section{[C $_{5}$ MPyr $]^{+}$}

SCF Energy = -448.4756604095

$\begin{array}{cccc}4.99832045719832 & -0.67937013868966 & 0.46067508864160 & \mathrm{c} \\ -2.37488272068358 & 0.25582135417005 & 0.49127594099749 & \mathrm{n} \\ -4.38146959701593 & -1.24299847992326 & 1.94027646166754 & \mathrm{c} \\ -3.11874562646357 & -0.23808867053650 & -2.25749419846950 & \mathrm{c} \\ -6.85279662243537 & -0.88505556062451 & 0.45590349706468 & \mathrm{c} \\ 7.28576746275099 & 0.62322875785902 & -0.77461506395377 & \mathrm{c} \\ 0.27031979965375 & -0.71561318538933 & 1.12972522196214 & \mathrm{c} \\ 2.46338076390891 & 0.58822986678868 & -0.22540676150420 & \mathrm{c} \\ -6.02518298622577 & -0.21691354862130 & -2.29185173114038 & \mathrm{c} \\ -2.61219590366477 & 3.02362694748635 & 1.10875671866994 & \mathrm{c} \\ 5.23909523761745 & -0.67433739393113 & 2.55511992293548 & \mathrm{~h} \\ 4.94679171793506 & -2.70509283783135 & -0.11917351709465 & \mathrm{~h} \\ -4.42236256676050 & -0.57321437486377 & 3.92384942584368 & \mathrm{~h} \\ -3.73749536442116 & -3.23719821295762 & 1.92431548251310 & \mathrm{~h} \\ -2.21107149851173 & 1.18908190203307 & -3.48853558396787 & \mathrm{~h} \\ -2.34277021199159 & -2.12531834179194 & -2.73560750947547 & \mathrm{~h} \\ -8.03663644508598 & 0.63221753938071 & 1.28770897377421 & \mathrm{~h} \\ -7.99308909655902 & -2.64006385395776 & 0.54172988369570 & \mathrm{~h} \\ 7.02198475704013 & 0.64613265088839 & -2.86537133780075 & \mathrm{~h} \\ 7.33766084423368 & 2.64188774889215 & -0.17266626317078 & \mathrm{~h} \\ 0.23941471149260 & -2.76590310321705 & 0.67715651180035 & \mathrm{~h} \\ 0.45509843847763 & -0.52873491920155 & 3.21335214597461 & \mathrm{~h} \\ 2.56196245479983 & 2.62073372397068 & 0.30468529889854 & \mathrm{~h} \\ 2.19396140976647 & 0.50263571553216 & -2.31141436894032 & \mathrm{~h} \\ -6.72906490285525 & -1.60187151909254 & -3.69747373384938 & \mathrm{~h} \\ -6.76696731509816 & 1.65378488713924 & -2.87754160939996 & \mathrm{~h} \\ -4.59360875119611 & 3.63073687709860 & 0.82840138146797 & \mathrm{~h} \\ -1.35874323361936 & 4.12778962864924 & -0.14794847655078 & \mathrm{~h} \\ -2.06005916667126 & 3.32131130935265 & 3.10688981818874 & \mathrm{~h} \\ 9.80539291528947 & -0.65255881468203 & -0.12530886026181 & \mathrm{c} \\ 9.84976171866675 & -2.64941625546891 & -0.78286806087344 & \mathrm{~h} \\ 10.15645413027619 & -0.65522706395318 & 1.94813176535659 & \mathrm{~h}\end{array}$


S-24

\section{$\begin{array}{lllll}11.41107308648466 & 0.34818659389677 & -1.03597155307729 & \mathrm{~h}\end{array}$}

$\begin{array}{cccc}\text { [C }_{\mathbf{5}} \mathbf{N E t}_{\mathbf{3}} \mathbf{H}^{+} & & & \\ \text {SCF Energy }=-488.9473184069 & & \\ 0.20927637839890 & -1.25770261344418 & -0.12862428329528 & \mathrm{c} \\ -2.52410139860801 & -0.23145091964462 & -0.14437724257911 & \mathrm{n} \\ -3.37662375048166 & 0.62131872971378 & 2.49182905585477 & \mathrm{c} \\ -4.28416954628410 & -2.39587919336279 & -0.98534578390825 & \mathrm{c} \\ -2.73778724561188 & 1.91447053380481 & -2.07992455708876 & \mathrm{c} \\ 2.41102953511100 & 0.60523244224829 & -0.39626129061878 & \mathrm{c} \\ -4.14769583576308 & -4.85097315169579 & 0.52275233333735 & \mathrm{c} \\ -5.34893294467276 & 3.08335134125910 & -2.43713127547161 & \mathrm{c} \\ -2.03521396419508 & 2.87664025465137 & 3.67987910945837 & \mathrm{c} \\ 7.25679267072483 & 0.91092312286802 & -0.45491242314104 & \mathrm{c} \\ 4.94269359226899 & -0.80649173842413 & -0.06207789471060 & \mathrm{c} \\ 9.77539842387712 & -0.47931945281111 & -0.09837200318384 & \mathrm{c} \\ 0.38791468945492 & -2.34193278582324 & 1.65593661049080 & \mathrm{~h} \\ 0.29455017706990 & -2.63576765118228 & -1.70917994111086 & \mathrm{~h} \\ -5.42792141641343 & 1.00743257700214 & 2.31153360964478 & \mathrm{~h} \\ -3.16950624204457 & -1.06285916604719 & 3.71656251961725 & \mathrm{~h} \\ -3.80763154586354 & -2.74182950774422 & -2.99907268331613 & \mathrm{~h} \\ -6.22528128027817 & -1.61722567871247 & -0.91527576996321 & \mathrm{~h} \\ -2.02955471065810 & 1.10691544163791 & -3.88189149287470 & \mathrm{~h} \\ -1.37266178950151 & 3.37543822832488 & -1.47390426054885 & \mathrm{~h} \\ 2.30394514189108 & 2.15502810551581 & 1.01442777222468 & \mathrm{~h} \\ 2.39050909356984 & 1.50244140883336 & -2.29600407260624 & \mathrm{~h} \\ -5.51040974531745 & -6.18499969500466 & -0.35761319846941 & \mathrm{~h} \\ -4.73250027344363 & -4.63396957792376 & 2.52550679545701 & \mathrm{~h} \\ -2.26558185401413 & -5.77158032009067 & 0.45287224762915 & \mathrm{~h} \\ -5.14885910329553 & 4.61321278568273 & -3.86210154422916 & \mathrm{~h} \\ -6.09909160574175 & 3.97855570568292 & -0.69443990843028 & \mathrm{~h} \\ -6.77801389205032 & 1.74607168083466 & -3.18907028332999 & \mathrm{~h} \\ -3.03553292239261 & 3.30807773524257 & 5.47600632680956 & \mathrm{~h} \\ -0.04012294086334 & 2.48001724460506 & 4.17744467117441 & \mathrm{~h} \\ -2.11691916253302 & 4.61504543710736 & 2.50912102307084 & \mathrm{~h} \\ 7.17627606544080 & 1.74585065777719 & -2.38790765327370 & \mathrm{~h} \\ 7.14878172752982 & 2.53478426124426 & 0.88430434366557 & \mathrm{~h} \\ 5.03395958939931 & -2.42022904467767 & -1.41668570611961 & \mathrm{~h} \\ 9.94853072715599 & -1.27405833718106 & 1.84096715826213 & \mathrm{~h}\end{array}$

\section{Vibrational Frequencies}

Calculated using the AOFORCE module of TURBOMOLE

(RI)-BP86/SV(P) level 


\section{Anions:}

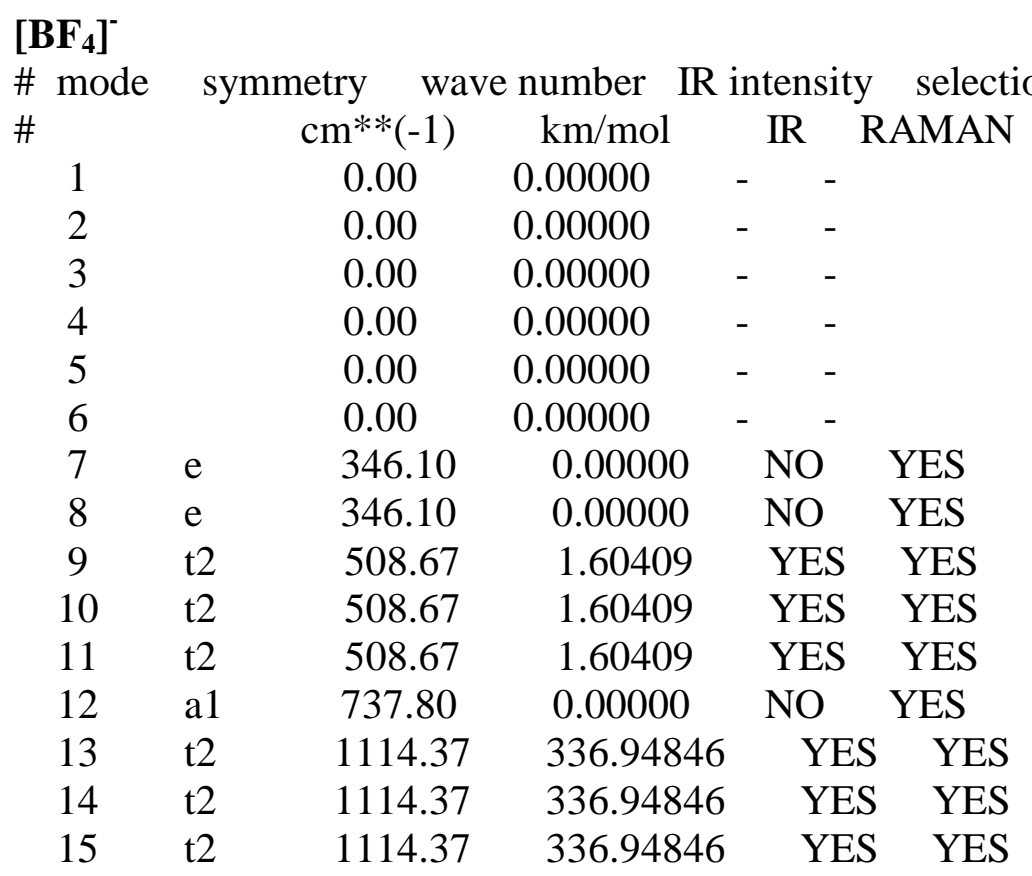

\section{$\left[\mathrm{PF}_{6}\right]^{-}$}

\# mode symmetry wave number IR intensity selection rules

$\begin{array}{ccccccc}\# & & \mathrm{~cm} * *(-1) & \mathrm{km} / \mathrm{mol} & \mathrm{IR} & \mathrm{RAMAN} \\ 1 & & 0.00 & 0.00000 & - & - & \\ 2 & & 0.00 & 0.00000 & - & - & \\ 3 & & 0.00 & 0.00000 & - & - & \\ 4 & & 0.00 & 0.00000 & - & - & \\ 5 & & 0.00 & 0.00000 & - & - & \\ 6 & & 0.00 & 0.00000 & - & \\ 7 & \mathrm{~b} 2 \mathrm{u} & 287.45 & 0.00000 & \text { NO } & \text { NO } \\ 8 & \text { eu } & 287.45 & 0.00000 & \text { YES } & \text { NO } \\ 9 & \text { eu } & 287.45 & 0.00000 & \text { YES } & \text { NO } \\ 10 & \text { b2g } & 430.24 & 0.00000 & \text { NO } & \text { YES } \\ 11 & \text { eg } & 430.24 & 0.00000 & \text { NO } & \text { YES } \\ 12 & \text { eg } & 430.24 & 0.00000 & \text { NO } & \text { YES } \\ 13 & \text { a2u } & 524.22 & 27.14714 & \text { YES } & \text { NO } \\ 14 & \text { eu } & 524.22 & 27.14717 & \text { YES } & \text { NO } \\ 15 & \text { eu } & 524.22 & 27.14717 & \text { YES } & \text { NO } \\ 16 & \text { a1g } & 581.21 & 0.00000 & \text { NO } & \text { YES } \\ 17 & \text { b1g } & 581.21 & 0.00000 & \text { NO } & \text { YES } \\ 18 & \text { a1g } & 698.09 & 0.00000 & \text { NO } & \text { YES } \\ 19 & \text { a2u } & 899.50 & 405.42973 & \text { YES } & \text { NO } \\ 20 & \text { eu } & 899.50 & 405.42968 & \text { YES } & \text { NO } \\ 21 & \text { eu } & 899.50 & 405.42968 & \text { YES } & \text { NO }\end{array}$




\section{[TfO]}

\# mode symmetry wave number IR intensity selection rules

\#

1

2

3

4

5

6

7

$7 \quad \mathrm{a}$

8 a

$9 \quad \mathrm{a}$

$10 \quad \mathrm{a}$

11 a

12 a

13 a

14 a

15 a

16 a

$17 \quad \mathrm{a}$

18 a

19 a

20 a

21 a

22 a

23 a

24 a

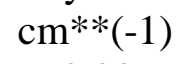

0.00

0.00

0.00

0.00

0.00

52.94

176.01

177.13

261.60

314.48

315.31

477.78

477.97

536.81

537.63

591.00

711.30

963.07

1122.15

1123.24

1152.22

1217.40

1218.84 $\mathrm{km} / \mathrm{mol}$ IR RAMAN $0.00000 \quad-\quad-$ $0.00000-\quad-$

0.00000

0.00000

0.00000

0.00000

0.00084

$$
1.03317
$$

$$
1.02109
$$

$$
0.04372
$$

0.09795

0.08004

16.46249

16.67142

5.91424

5.76696

160.44235

1.36687

162.69661

153.63557

153.63110

115.17588

338.43999

337.89691
YES YES

YES YES

YES YES

YES YES

YES YES

YES YES

YES YES

YES YES

YES YES

YES YES

YES YES

YES YES

YES YES

YES YES

YES YES

YES YES

YES YES

YES YES

$\left[\mathbf{T f}_{\mathbf{2}} \mathbf{N}\right]^{-}$

\#

1

2

3

4

5

6

$7 \quad \mathrm{a}$

8 a

$9 \quad \mathrm{a}$

10 a

$11 \quad \mathrm{a}$

12 a

13 a

$14 \mathrm{a}$

15 a

16 a

$17 \quad \mathrm{a}$

$18 \quad \mathrm{a}$ symmetry wave number IR intensity selection rules

$\begin{array}{ccccc}\mathrm{cm} * *(-1) & \mathrm{km} / \mathrm{mol} & \text { IR } & \text { RAMAN } \\ 0.00 & 0.00000 & - & - & \\ 0.00 & 0.00000 & - & - & \\ 0.00 & 0.00000 & - & - & \\ 0.00 & 0.00000 & - & - & \\ 0.00 & 0.00000 & - & - & \\ 0.00 & 0.00000 & - & - & \\ 22.64 & 0.97017 & \text { YES } & \text { YES } \\ 29.33 & 0.44481 & \text { YES } & \text { YES } \\ 44.44 & 0.61623 & \text { YES } & \text { YES } \\ 65.52 & 1.26393 & \text { YES } & \text { YES } \\ 103.51 & 0.05869 & \text { YES } & \text { YES } \\ 136.15 & 0.19742 & \text { YES } & \text { YES } \\ 178.92 & 1.48539 & \text { YES } & \text { YES } \\ 195.21 & 1.09874 & \text { YES } & \text { YES } \\ 201.77 & 6.95918 & \text { YES } & \text { YES } \\ 243.75 & 0.07801 & \text { YES } & \text { YES } \\ 265.08 & 0.15084 & \text { YES } & \text { YES } \\ 278.90 & 0.32321 & \text { YES } & \text { YES }\end{array}$




$\begin{array}{llcccc}19 & \mathrm{a} & 283.72 & 0.37154 & \text { YES } & \text { YES } \\ 20 & \mathrm{a} & 307.72 & 0.24996 & \text { YES } & \text { YES } \\ 21 & \mathrm{a} & 336.88 & 5.56497 & \text { YES } & \text { YES } \\ 22 & \mathrm{a} & 357.90 & 6.42769 & \text { YES } & \text { YES } \\ 23 & \mathrm{a} & 373.48 & 1.93926 & \text { YES } & \text { YES } \\ 24 & \mathrm{a} & 476.57 & 82.59380 & \text { YES } & \text { YES } \\ 25 & \mathrm{a} & 505.02 & 0.62360 & \text { YES } & \text { YES } \\ 26 & \mathrm{a} & 525.44 & 2.33833 & \text { YES } & \text { YES } \\ 27 & \mathrm{a} & 533.62 & 0.92658 & \text { YES } & \text { YES } \\ 28 & \mathrm{a} & 540.73 & 28.68992 & \text { YES } & \text { YES } \\ 29 & \mathrm{a} & 542.25 & 7.68079 & \text { YES } & \text { YES } \\ 30 & \mathrm{a} & 566.58 & 102.41168 & \text { YES } & \text { YES } \\ 31 & \mathrm{a} & 581.28 & 212.33704 & \text { YES } & \text { YES } \\ 32 & \mathrm{a} & 698.37 & 34.19459 & \text { YES } & \text { YES } \\ 33 & \mathrm{a} & 725.51 & 0.46167 & \text { YES } & \text { YES } \\ 34 & \mathrm{a} & 741.79 & 16.88165 & \text { YES } & \text { YES } \\ 35 & \mathrm{a} & 948.03 & 561.04532 & \text { YES } & \text { YES } \\ 36 & \mathrm{a} & 1074.74 & 220.25074 & \text { YES } & \text { YES } \\ 37 & \mathrm{a} & 1076.23 & 53.14865 & \text { YES } & \text { YES } \\ 38 & \mathrm{a} & 1140.03 & 311.39788 & \text { YES } & \text { YES } \\ 39 & \mathrm{a} & 1154.53 & 28.21182 & \text { YES } & \text { YES } \\ 40 & \mathrm{a} & 1165.68 & 141.29664 & \text { YES } & \text { YES } \\ 41 & \mathrm{a} & 1168.72 & 399.31667 & \text { YES } & \text { YES } \\ 42 & \mathrm{a} & 1186.00 & 228.98294 & \text { YES } & \text { YES } \\ 43 & \mathrm{a} & 1189.10 & 129.25459 & \text { YES } & \text { YES } \\ 44 & \mathrm{a} & 1266.55 & 78.49367 & \text { YES } & \text { YES } \\ 45 & \mathrm{a} & 1287.14 & 398.58909 & \text { YES } & \text { YES }\end{array}$




\section{Cations:}

\section{[EMIM] $^{+}$}

\# mode symmetry wave number IR intensity selection rules

\#

1

3

3
4

5

6

$7 \quad \mathrm{a}$

8 a

9 a

$10 \quad \mathrm{a}$

11 a

$12 \mathrm{a}$

13 a

14 a

15 a

16 a

$17 \quad \mathrm{a}$

18 a

19 a

20 a

21 a

$22 \quad \mathrm{a}$

23 a

24 a

25 a

26 a

$27 \quad \mathrm{a}$

28 a

29 a

30 a

31 a

32 a

33 a

34 a

35 a

36 a

$37 \quad \mathrm{a}$

38 a

39 a

40 a

$41 \quad \mathrm{a}$

$42 \quad \mathrm{a}$

43 a

$44 \quad \mathrm{a}$ $\mathrm{cm}^{* *}(-1)$

$$
0.00
$$

0.00

0.00

0.00

0.00

0.00

49.82

68.62

134.41

208.20

233.46

291.39

375.32

418.80

585.09

621.12

649.40

691.92

710.46

780.85

784.56

837.78

946.55

1011.21

1020.77

1064.55

1075.47

1085.17

1100.31

1109.58

1146.38

1228.10

1270.39

1320.94

1345.06

1372.05

1385.79

1400.14

1416.76

1424.57

1431.23

1433.26

1447.80

1453.71 $\mathrm{km} / \mathrm{mol}$

0.00000

0.00000

0.00000

0.00000

0.00000

0.00000

0.23761

0.12277

1.19938

0.20739

1.94580

0.17945

0.39164

0.33364

1.41306

9.42578

13.74506

5.84272

18.47745

34.47045

7.19732

0.04510

4.30217

0.60177

3.23458

4.38898

7.30090

8.55467

2.16674

1.74758

88.18383

0.12759

0.84234

12.04371

10.35885

7.51190

6.58165

1.59800

2.37674

19.97442

10.89562

19.60038

16.66360

10.81481
IR RAMAN

-

$-$

-

-

YES YES

YES YES

YES YES

YES YES

YES YES

YES YES

YES YES

YES YES

YES YES

YES YES

YES YES

YES YES

YES YES

YES YES

YES YES

YES YES

YES YES

YES YES

YES YES

YES YES

YES YES

YES YES

YES YES

YES YES

YES YES

YES YES

YES YES

YES YES

YES YES

YES YES

YES YES

YES YES

YES YES

YES YES

YES YES

YES YES

YES YES

YES YES 


$\begin{array}{lllllc}45 & \mathrm{a} & 1563.93 & 34.68039 & \text { YES } & \text { YES } \\ 46 & \mathrm{a} & 1571.20 & 37.13646 & \text { YES } & \text { YES } \\ 47 & \mathrm{a} & 2965.26 & 2.36858 & \text { YES } & \text { YES } \\ 48 & \mathrm{a} & 2987.22 & 2.31780 & \text { YES } & \text { YES } \\ 49 & \mathrm{a} & 3000.12 & 4.88435 & \text { YES } & \text { YES } \\ 50 & \mathrm{a} & 3048.02 & 0.93607 & \text { YES } & \text { YES } \\ 51 & \mathrm{a} & 3062.34 & 1.61643 & \text { YES } & \text { YES } \\ 52 & \mathrm{a} & 3068.31 & 3.85791 & \text { YES } & \text { YES } \\ 53 & \mathrm{a} & 3084.90 & 0.36471 & \text { YES } & \text { YES } \\ 54 & \mathrm{a} & 3105.24 & 0.22178 & \text { YES } & \text { YES } \\ 55 & \mathrm{a} & 3198.63 & 16.93090 & \text { YES } & \text { YES } \\ 56 & \mathrm{a} & 3204.50 & 33.63864 & \text { YES } & \text { YES } \\ 57 & \mathrm{a} & 3215.19 & 11.18646 & \text { YES } & \text { YES }\end{array}$

\section{$\left[\mathrm{C}_{3} \mathrm{MIM}\right]^{+}$}

\# mode symmetry wave number IR intensity selection rules

\#

1

2

3

4

5

6

7 a

8 a

$9 \quad \mathrm{a}$

$10 \quad \mathrm{a}$

11 a

12 a

13 a

14 a

15 a

16 a

$17 \quad \mathrm{a}$

$18 \quad \mathrm{a}$

19 a

20 a

21 a

$22 \quad \mathrm{a}$

23 a

24 a

25 a

26 a

$27 \quad \mathrm{a}$

28 a

$29 \quad \mathrm{a}$

30 a

31 a

32 a

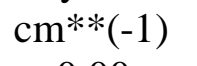

\subsection{0} 0.00000

0.13064 YES YES

0.04913 YES YES

0.35194 YES YES

2.87081 YES YES

0.04301 YES YES

0.30193 YES YES

0.21234 YES YES

0.28297 YES YES

0.31919 YES YES

0.75607 YES YES

15.13563 YES YES

8.29295 YES YES

15.75913 YES YES

13.62120 YES YES

2.92152 YES YES

36.16387 YES YES

0.07446 YES YES

1.32659 YES YES

5.40068 YES YES

1.64151 YES YES

1.94794 YES YES

0.65193 YES YES

5.75656 YES YES

11.30669 YES YES

4.44599 YES YES


S-30

$\begin{array}{llcccc}33 & \mathrm{a} & 1109.24 & 1.71168 & \text { YES } & \text { YES } \\ 34 & \mathrm{a} & 1109.89 & 0.15558 & \text { YES } & \text { YES } \\ 35 & \mathrm{a} & 1144.55 & 79.11268 & \text { YES } & \text { YES } \\ 36 & \mathrm{a} & 1211.93 & 0.00921 & \text { YES } & \text { YES } \\ 37 & \mathrm{a} & 1255.01 & 0.96313 & \text { YES } & \text { YES } \\ 38 & \mathrm{a} & 1288.28 & 2.72879 & \text { YES } & \text { YES } \\ 39 & \mathrm{a} & 1295.50 & 6.64798 & \text { YES } & \text { YES } \\ 40 & \mathrm{a} & 1325.23 & 4.72419 & \text { YES } & \text { YES } \\ 41 & \mathrm{a} & 1357.87 & 2.65205 & \text { YES } & \text { YES } \\ 42 & \mathrm{a} & 1371.73 & 2.57201 & \text { YES } & \text { YES } \\ 43 & \mathrm{a} & 1384.95 & 5.63442 & \text { YES } & \text { YES } \\ 44 & \mathrm{a} & 1402.09 & 2.79849 & \text { YES } & \text { YES } \\ 45 & \mathrm{a} & 1416.37 & 2.21188 & \text { YES } & \text { YES } \\ 46 & \mathrm{a} & 1424.44 & 19.47195 & \text { YES } & \text { YES } \\ 47 & \mathrm{a} & 1430.32 & 12.61229 & \text { YES } & \text { YES } \\ 48 & \mathrm{a} & 1435.86 & 3.04995 & \text { YES } & \text { YES } \\ 49 & \mathrm{a} & 1440.50 & 12.23967 & \text { YES } & \text { YES } \\ 50 & \mathrm{a} & 1452.37 & 20.61251 & \text { YES } & \text { YES } \\ 51 & \mathrm{a} & 1455.50 & 11.04783 & \text { YES } & \text { YES } \\ 52 & \mathrm{a} & 1564.28 & 35.87754 & \text { YES } & \text { YES } \\ 53 & \mathrm{a} & 1570.23 & 38.95853 & \text { YES } & \text { YES } \\ 54 & \mathrm{a} & 2952.40 & 1.84311 & \text { YES } & \text { YES } \\ 55 & \mathrm{a} & 2956.92 & 22.34231 & \text { YES } & \text { YES } \\ 56 & \mathrm{a} & 2987.82 & 2.53313 & \text { YES } & \text { YES } \\ 57 & \mathrm{a} & 2994.97 & 8.32406 & \text { YES } & \text { YES } \\ 58 & \mathrm{a} & 2999.62 & 4.04978 & \text { YES } & \text { YES } \\ 59 & \mathrm{a} & 3039.19 & 12.70916 & \text { YES } & \text { YES } \\ 60 & \mathrm{a} & 3055.97 & 4.43064 & \text { YES } & \text { YES } \\ 61 & \mathrm{a} & 3056.32 & 5.09576 & \text { YES } & \text { YES } \\ 62 & \mathrm{a} & 3084.76 & 0.33802 & \text { YES } & \text { YES } \\ 63 & \mathrm{a} & 3107.00 & 0.22898 & \text { YES } & \text { YES } \\ 64 & \mathrm{a} & 3199.69 & 16.96227 & \text { YES } & \text { YES } \\ 65 & \mathrm{a} & 3206.26 & 32.45742 & \text { YES } & \text { YES } \\ 66 & \mathrm{a} & 3216.55 & 10.88973 & \text { YES } & \text { YES }\end{array}$

\section{[BMIM] $^{+}$}

\# mode symmetry wave number IR intensity selection rules

$\begin{array}{ccccccc}\# & & \mathrm{~cm} * *(-1) & \mathrm{km} / \mathrm{mol} & \mathrm{IR} & \text { RAMAN } \\ 1 & & 0.00 & 0.00000 & - & - & \\ 2 & & 0.00 & 0.00000 & - & - & \\ 3 & & 0.00 & 0.00000 & - & - & \\ 4 & & 0.00 & 0.00000 & - & - & \\ 5 & & 0.00 & 0.00000 & - & - & \\ 6 & & 0.00 & 0.00000 & - & - & \\ 7 & \mathrm{a} & 41.20 & 0.40656 & \text { YES } & \text { YES } \\ 8 & \mathrm{a} & 69.92 & 0.31914 & \text { YES } & \text { YES } \\ 9 & \mathrm{a} & 74.84 & 0.02253 & \text { YES } & \text { YES } \\ 10 & \mathrm{a} & 82.36 & 0.16413 & \text { YES } & \text { YES } \\ 11 & \mathrm{a} & 125.73 & 0.06846 & \text { YES } & \text { YES }\end{array}$


S-31

\begin{tabular}{|c|c|c|c|c|c|}
\hline 12 & $\mathrm{a}$ & 197.30 & 2.13275 & YES & YES \\
\hline 13 & $\mathrm{a}$ & 242.87 & 0.11557 & YES & YES \\
\hline 14 & $\mathrm{a}$ & 247.37 & 0.55923 & YES & YES \\
\hline 15 & $\mathrm{a}$ & 274.40 & 0.28540 & YES & YES \\
\hline 16 & $\mathrm{a}$ & 315.34 & 0.22086 & YES & YES \\
\hline 17 & $\mathrm{a}$ & 403.62 & 0.54999 & YES & YES \\
\hline 18 & $\mathrm{a}$ & 425.05 & 1.02836 & YES & YES \\
\hline 19 & $\mathrm{a}$ & 615.87 & 0.60947 & YES & YES \\
\hline 20 & $\mathrm{a}$ & 622.61 & 15.00729 & YES & YES \\
\hline 21 & $\mathrm{a}$ & 655.37 & 9.88600 & YES & YES \\
\hline 22 & $\mathrm{a}$ & 709.43 & 16.44986 & YES & YES \\
\hline 23 & $\mathrm{a}$ & 726.10 & 11.12040 & YES & YES \\
\hline 24 & $\mathrm{a}$ & 734.01 & 3.71856 & YES & YES \\
\hline 25 & $\mathrm{a}$ & 780.21 & 36.23473 & YES & YES \\
\hline 26 & $\mathrm{a}$ & 786.62 & 1.00803 & YES & YES \\
\hline 27 & $\mathrm{a}$ & 836.65 & 0.06925 & YES & YES \\
\hline 28 & $\mathrm{a}$ & 900.37 & 0.69455 & YES & YES \\
\hline 29 & $\mathrm{a}$ & 923.93 & 0.44180 & YES & YES \\
\hline 30 & $\mathrm{a}$ & 999.57 & 2.60126 & YES & YES \\
\hline 31 & $\mathrm{a}$ & 1012.18 & 1.31667 & YES & YES \\
\hline 32 & $\mathrm{a}$ & 1020.16 & 2.30462 & YES & YES \\
\hline 33 & $\mathrm{a}$ & 1048.37 & 0.53295 & YES & YES \\
\hline 34 & $\mathrm{a}$ & 1067.17 & 6.43623 & YES & YES \\
\hline 35 & $\mathrm{a}$ & 1085.08 & 11.48745 & YES & YES \\
\hline 36 & $\mathrm{a}$ & 1100.47 & 9.90073 & YES & YES \\
\hline 37 & $\mathrm{a}$ & 1110.09 & 0.87072 & YES & YES \\
\hline 38 & $\mathrm{a}$ & 1115.02 & 0.58353 & YES & YES \\
\hline 39 & $\mathrm{a}$ & 1144.22 & 81.29280 & YES & YES \\
\hline 40 & $\mathrm{a}$ & 1197.63 & 0.16896 & YES & YES \\
\hline 41 & $\mathrm{a}$ & 1246.77 & 0.84626 & YES & YES \\
\hline 42 & $\mathrm{a}$ & 1254.80 & 3.66135 & YES & YES \\
\hline 43 & $\mathrm{a}$ & 1279.52 & 0.29285 & YES & YES \\
\hline 44 & $\mathrm{a}$ & 1292.82 & 3.84568 & YES & YES \\
\hline 45 & $\mathrm{a}$ & 1323.37 & 5.00552 & YES & YES \\
\hline 46 & $\mathrm{a}$ & 1341.34 & 0.93139 & YES & YES \\
\hline 47 & $\mathrm{a}$ & 1360.06 & 7.31204 & YES & YES \\
\hline 48 & $\mathrm{a}$ & 1376.12 & 2.53592 & YES & YES \\
\hline 49 & $\mathrm{a}$ & 1384.93 & 5.50463 & YES & YES \\
\hline 50 & $\mathrm{a}$ & 1402.72 & 2.64169 & YES & YES \\
\hline 51 & $\mathrm{a}$ & 1417.16 & 2.11162 & YES & YES \\
\hline 52 & $\mathrm{a}$ & 1424.75 & 19.06802 & YES & YES \\
\hline 53 & $\mathrm{a}$ & 1428.82 & 9.04092 & YES & YES \\
\hline 54 & $\mathrm{a}$ & 1431.66 & 0.96359 & YES & YES \\
\hline 55 & $\mathrm{a}$ & 1441.94 & 6.74054 & YES & YES \\
\hline 56 & $\mathrm{a}$ & 1442.26 & 13.65071 & YES & YES \\
\hline 57 & $\mathrm{a}$ & 1452.02 & 15.31110 & YES & YES \\
\hline 58 & $\mathrm{a}$ & 1455.90 & 12.46562 & YES & YES \\
\hline 59 & $\mathrm{a}$ & 1563.63 & 38.57502 & YES & YES \\
\hline 60 & $\mathrm{a}$ & 1570.10 & 36.97627 & YES & YES \\
\hline
\end{tabular}




$\begin{array}{llcccc}61 & \mathrm{a} & 2937.03 & 4.09154 & \text { YES } & \text { YES } \\ 62 & \mathrm{a} & 2946.26 & 18.07023 & \text { YES } & \text { YES } \\ 63 & \mathrm{a} & 2950.98 & 29.93418 & \text { YES } & \text { YES } \\ 64 & \mathrm{a} & 2976.89 & 1.17830 & \text { YES } & \text { YES } \\ 65 & \mathrm{a} & 2986.85 & 2.74400 & \text { YES } & \text { YES } \\ 66 & \mathrm{a} & 2994.44 & 8.34793 & \text { YES } & \text { YES } \\ 67 & \mathrm{a} & 2999.47 & 15.67012 & \text { YES } & \text { YES } \\ 68 & \mathrm{a} & 3033.19 & 24.40208 & \text { YES } & \text { YES } \\ 69 & \mathrm{a} & 3047.27 & 10.17180 & \text { YES } & \text { YES } \\ 70 & \mathrm{a} & 3054.61 & 3.82959 & \text { YES } & \text { YES } \\ 71 & \mathrm{a} & 3083.59 & 0.27859 & \text { YES } & \text { YES } \\ 72 & \mathrm{a} & 3106.38 & 0.20305 & \text { YES } & \text { YES } \\ 73 & \mathrm{a} & 3199.06 & 16.34586 & \text { YES } & \text { YES } \\ 74 & \mathrm{a} & 3204.31 & 31.63345 & \text { YES } & \text { YES } \\ 75 & \mathrm{a} & 3215.55 & 11.35726 & \text { YES } & \text { YES }\end{array}$

\section{$\left[\mathrm{C}_{5} \mathrm{MIM}\right]^{+}$}

\# mode symmetry wave number IR intensity selection rules

\#

1

3

4

5

6

$7 \quad a$

$8 \quad \mathrm{a}$

$9 a$

$10 \mathrm{a}$

11 a

$12 a$

13 a

14 a

15 a

16 a

$17 \quad \mathrm{a}$

18 a

19 a

20 a

$21 \quad \mathrm{a}$

$22 \quad a$

23 a

24 a

25 a

26 a

$27 \quad \mathrm{a}$

28 a

29 a

30 a $\mathrm{cm}^{* *}(-1)$

$$
0.00
$$$$
0.00
$$$$
0.00
$$$$
0.00
$$$$
0.00
$$$$
0.00
$$

35.53

49.88

60.32

71.66

109.26

134.96

157.94

220.67

248.63

266.64

294.01

343.71

407.37

452.87

615.45

622.09

657.58

709.12

726.01

729.73

752.48

780.63

833.47

836.62 $\mathrm{km} / \mathrm{mol}$

0.00000

0.00000

0.00000

0.00000

0.00000

0.00000

0.39295

0.02252

0.31411

0.04257

0.14677

0.02252

1.18089

1.91207

0.00233

0.52955

0.27727

0.71532

0.19079

0.90019

0.83604

14.81805

10.17463

16.30362

11.14317

4.35740

0.37657

36.15474

0.63778

0.04762
IR RAMAN

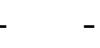

-

$-$

-

- -

YES YES

YES YES

YES YES

YES YES

YES YES

YES YES

YES YES

YES YES

YES YES

YES YES

YES YES

YES YES

YES YES

YES YES

YES YES

YES YES

YES YES

YES YES

YES YES

YES YES

YES YES

YES YES

YES YES

YES YES 


\begin{tabular}{|c|c|c|c|c|c|}
\hline 31 & $\mathrm{a}$ & 891.80 & 0.95778 & YES & YES \\
\hline 32 & $\mathrm{a}$ & 957.60 & 0.66631 & YES & YES \\
\hline 33 & $\mathrm{a}$ & 1003.30 & 2.70719 & YES & YES \\
\hline 34 & $\mathrm{a}$ & 1010.51 & 1.55127 & YES & YES \\
\hline 35 & $\mathrm{a}$ & 1014.43 & 2.11399 & YES & YES \\
\hline 36 & $\mathrm{a}$ & 1029.34 & 1.75325 & YES & YES \\
\hline 37 & $\mathrm{a}$ & 1053.91 & 1.58151 & YES & YES \\
\hline 38 & $\mathrm{a}$ & 1065.75 & 6.50875 & YES & YES \\
\hline 39 & $\mathrm{a}$ & 1084.71 & 11.95363 & YES & YES \\
\hline 40 & $\mathrm{a}$ & 1104.63 & 5.40772 & YES & YES \\
\hline 41 & $\mathrm{a}$ & 1110.02 & 0.79741 & YES & YES \\
\hline 42 & $\mathrm{a}$ & 1117.58 & 0.13007 & YES & YES \\
\hline 43 & $\mathrm{a}$ & 1144.06 & 84.46004 & YES & YES \\
\hline 44 & $\mathrm{a}$ & 1188.28 & 0.08486 & YES & YES \\
\hline 45 & $\mathrm{a}$ & 1232.90 & 3.19447 & YES & YES \\
\hline 46 & $\mathrm{a}$ & 1239.27 & 0.09930 & YES & YES \\
\hline 47 & $\mathrm{a}$ & 1266.02 & 0.69001 & YES & YES \\
\hline 48 & $\mathrm{a}$ & 1288.83 & 1.42562 & YES & YES \\
\hline 49 & $\mathrm{a}$ & 1297.24 & 2.41640 & YES & YES \\
\hline 50 & $\mathrm{a}$ & 1309.04 & 0.50277 & YES & YES \\
\hline 51 & $\mathrm{a}$ & 1324.10 & 5.23759 & YES & YES \\
\hline 52 & $\mathrm{a}$ & 1352.99 & 8.20584 & YES & YES \\
\hline 53 & $\mathrm{a}$ & 1364.85 & 0.17878 & YES & YES \\
\hline 54 & $\mathrm{a}$ & 1372.17 & 3.39557 & YES & YES \\
\hline 55 & $\mathrm{a}$ & 1384.87 & 5.50009 & YES & YES \\
\hline 56 & $\mathrm{a}$ & 1401.32 & 2.74566 & YES & YES \\
\hline 57 & $\mathrm{a}$ & 1416.34 & 2.36157 & YES & YES \\
\hline 58 & $\mathrm{a}$ & 1423.98 & 19.51638 & YES & YES \\
\hline 59 & $\mathrm{a}$ & 1427.98 & 4.23795 & YES & YES \\
\hline 60 & $\mathrm{a}$ & 1430.08 & 2.43004 & YES & YES \\
\hline 61 & $\mathrm{a}$ & 1435.30 & 9.13965 & YES & YES \\
\hline 62 & $\mathrm{a}$ & 1440.79 & 11.79496 & YES & YES \\
\hline 63 & $\mathrm{a}$ & 1445.53 & 1.38027 & YES & YES \\
\hline 64 & $\mathrm{a}$ & 1453.12 & 18.41680 & YES & YES \\
\hline 65 & $\mathrm{a}$ & 1455.26 & 11.35238 & YES & YES \\
\hline 66 & $\mathrm{a}$ & 1563.57 & 36.62380 & YES & YES \\
\hline 67 & $\mathrm{a}$ & 1570.43 & 40.91082 & YES & YES \\
\hline 68 & $\mathrm{a}$ & 2926.99 & 2.19046 & YES & YES \\
\hline 69 & $\mathrm{a}$ & 2936.21 & 18.85607 & YES & YES \\
\hline 70 & $\mathrm{a}$ & 2945.83 & 7.58504 & YES & YES \\
\hline 71 & $\mathrm{a}$ & 2947.12 & 54.35154 & YES & YES \\
\hline 72 & $\mathrm{a}$ & 2964.58 & 0.05852 & YES & YES \\
\hline 73 & $\mathrm{a}$ & 2983.47 & 8.17117 & YES & YES \\
\hline 74 & $\mathrm{a}$ & 2987.17 & 2.79276 & YES & YES \\
\hline 75 & $\mathrm{a}$ & 2993.73 & 8.45203 & YES & YES \\
\hline 76 & $\mathrm{a}$ & 3000.22 & 28.62618 & YES & YES \\
\hline 77 & $\mathrm{a}$ & 3029.07 & 30.56104 & YES & YES \\
\hline 78 & $\mathrm{a}$ & 3040.35 & 14.54956 & YES & YES \\
\hline 79 & $\mathrm{a}$ & 3054.11 & 3.72051 & YES & YES \\
\hline
\end{tabular}




$\begin{array}{lllccc}80 & \mathrm{a} & 3083.81 & 0.25970 & \text { YES } & \text { YES } \\ 81 & \mathrm{a} & 3106.02 & 0.19007 & \text { YES } & \text { YES } \\ 82 & \mathrm{a} & 3199.18 & 16.28702 & \text { YES } & \text { YES } \\ 83 & \mathrm{a} & 3204.93 & 31.52294 & \text { YES } & \text { YES } \\ 84 & \mathrm{a} & 3215.69 & 11.00187 & \text { YES } & \text { YES }\end{array}$

\section{[BMMIM] $^{+}$}

\# mode symmetry wave number IR intensity selection rules

\begin{tabular}{|c|c|c|c|c|c|}
\hline$\neq$ & & $\mathrm{cm}^{* *}(-1)$ & $\mathrm{km} / \mathrm{mol}$ & IR & RAMAN \\
\hline 1 & & 0.00 & 0.00000 & $-\quad-$ & \\
\hline 2 & & 0.00 & 0.00000 & - & \\
\hline 3 & & 0.00 & 0.00000 & - & \\
\hline 4 & & 0.00 & 0.00000 & - & \\
\hline 5 & & 0.00 & 0.00000 & - & \\
\hline 6 & & 0.00 & 0.00000 & - & \\
\hline 7 & $\mathrm{a}$ & 11.07 & 0.29510 & YES & YES \\
\hline 8 & $\mathrm{a}$ & 29.39 & 0.29511 & YES & YES \\
\hline 9 & $\mathrm{a}$ & 65.55 & 0.33108 & YES & YES \\
\hline 10 & $\mathrm{a}$ & 72.54 & 0.03018 & YES & YES \\
\hline 11 & $\mathrm{a}$ & 83.65 & 0.15950 & YES & YES \\
\hline 12 & $\mathrm{a}$ & 109.91 & 0.07119 & YES & YES \\
\hline 13 & $\mathrm{a}$ & 159.00 & 0.25749 & YES & YES \\
\hline 14 & $\mathrm{a}$ & 236.57 & 0.54254 & YES & YES \\
\hline 15 & $\mathrm{a}$ & 245.98 & 0.04929 & YES & YES \\
\hline 16 & $\mathrm{a}$ & 261.74 & 5.46057 & YES & YES \\
\hline 17 & $\mathrm{a}$ & 286.83 & 0.31433 & YES & YES \\
\hline 18 & $\mathrm{a}$ & 305.43 & 0.36016 & YES & YES \\
\hline 19 & $\mathrm{a}$ & 331.46 & 0.21467 & YES & YES \\
\hline 20 & $\mathrm{a}$ & 417.03 & 2.21878 & YES & YES \\
\hline 21 & $\mathrm{a}$ & 481.11 & 2.27382 & YES & YES \\
\hline 22 & $\mathrm{a}$ & 599.57 & 0.10345 & YES & YES \\
\hline 23 & $\mathrm{a}$ & 625.25 & 0.76205 & YES & YES \\
\hline 24 & $\mathrm{a}$ & 663.83 & 15.83623 & YES & YES \\
\hline 25 & $\mathrm{a}$ & 716.24 & 14.76299 & YES & YES \\
\hline 26 & $\mathrm{a}$ & 721.68 & 27.75416 & YES & YES \\
\hline 27 & $\mathrm{a}$ & 731.73 & 2.56614 & YES & YES \\
\hline 28 & $\mathrm{a}$ & 749.26 & 9.76752 & YES & YES \\
\hline 29 & $\mathrm{a}$ & 783.32 & 0.78937 & YES & YES \\
\hline 30 & $\mathrm{a}$ & 825.89 & 0.13746 & YES & YES \\
\hline 31 & $\mathrm{a}$ & 898.82 & 0.84108 & YES & YES \\
\hline 32 & $\mathrm{a}$ & 921.54 & 0.81589 & YES & YES \\
\hline 33 & $\mathrm{a}$ & 943.29 & 0.84376 & YES & YES \\
\hline 34 & $\mathrm{a}$ & 1004.35 & 0.89417 & YES & YES \\
\hline 35 & $\mathrm{a}$ & 1018.81 & 6.35126 & YES & YES \\
\hline 36 & $\mathrm{a}$ & 1032.85 & 1.68008 & YES & YES \\
\hline 37 & $\mathrm{a}$ & 1048.80 & 0.34880 & YES & YES \\
\hline 38 & $\mathrm{a}$ & 1055.81 & 6.43895 & YES & YES \\
\hline 39 & $\mathrm{a}$ & 1083.56 & 9.58568 & YES & YES \\
\hline 40 & $\mathrm{a}$ & 1100.98 & 4.94270 & YES & YES \\
\hline
\end{tabular}


S-35

\begin{tabular}{|c|c|c|c|c|c|}
\hline 41 & $\mathrm{a}$ & 1106.45 & 1.28391 & YES & YES \\
\hline 42 & $\mathrm{a}$ & 1119.62 & 14.46106 & YES & YES \\
\hline 43 & $\mathrm{a}$ & 1181.54 & 7.66386 & YES & YES \\
\hline 44 & $\mathrm{a}$ & 1216.35 & 17.80931 & YES & YES \\
\hline 45 & $\mathrm{a}$ & 1230.55 & 7.67491 & YES & YES \\
\hline 46 & $\mathrm{a}$ & 1253.89 & 1.76424 & YES & YES \\
\hline 47 & $\mathrm{a}$ & 1286.50 & 4.66209 & YES & YES \\
\hline 48 & $\mathrm{a}$ & 1293.06 & 2.85216 & YES & YES \\
\hline 49 & $\mathrm{a}$ & 1326.30 & 3.97721 & YES & YES \\
\hline 50 & $\mathrm{a}$ & 1341.17 & 0.51559 & YES & YES \\
\hline 51 & $\mathrm{a}$ & 1359.06 & 7.27649 & YES & YES \\
\hline 52 & $\mathrm{a}$ & 1371.68 & 4.46806 & YES & YES \\
\hline 53 & $\mathrm{a}$ & 1373.42 & 6.91487 & YES & YES \\
\hline 54 & $\mathrm{a}$ & 1381.09 & 6.12239 & YES & YES \\
\hline 55 & $\mathrm{a}$ & 1397.55 & 20.27767 & YES & YES \\
\hline 56 & $\mathrm{a}$ & 1410.90 & 28.45083 & YES & YES \\
\hline 57 & $\mathrm{a}$ & 1419.18 & 10.25389 & YES & YES \\
\hline 58 & $\mathrm{a}$ & 1429.17 & 15.26901 & YES & YES \\
\hline 59 & $\mathrm{a}$ & 1431.59 & 2.22706 & YES & YES \\
\hline 60 & $\mathrm{a}$ & 1435.58 & 8.19488 & YES & YES \\
\hline 61 & $\mathrm{a}$ & 1440.98 & 9.49930 & YES & YES \\
\hline 62 & $\mathrm{a}$ & 1441.64 & 7.66752 & YES & YES \\
\hline 63 & $\mathrm{a}$ & 1447.85 & 21.50381 & YES & YES \\
\hline 64 & $\mathrm{a}$ & 1452.28 & 12.67053 & YES & YES \\
\hline 65 & $\mathrm{a}$ & 1502.65 & 5.84572 & YES & YES \\
\hline 66 & $\mathrm{a}$ & 1522.01 & 59.67673 & YES & YES \\
\hline 67 & $\mathrm{a}$ & 1581.53 & 18.61523 & YES & YES \\
\hline 68 & $\mathrm{a}$ & 2936.48 & 4.83923 & YES & YES \\
\hline 69 & $\mathrm{a}$ & 2945.82 & 17.35277 & YES & YES \\
\hline 70 & $\mathrm{a}$ & 2950.51 & 30.55120 & YES & YES \\
\hline 71 & $\mathrm{a}$ & 2960.00 & 0.11713 & YES & YES \\
\hline 72 & $\mathrm{a}$ & 2975.94 & 1.25273 & YES & YES \\
\hline 73 & $\mathrm{a}$ & 2984.53 & 3.85689 & YES & YES \\
\hline 74 & $\mathrm{a}$ & 2995.32 & 9.60143 & YES & YES \\
\hline 75 & $\mathrm{a}$ & 3000.06 & 15.97195 & YES & YES \\
\hline 76 & $\mathrm{a}$ & 3032.84 & 24.64803 & YES & YES \\
\hline 77 & $\mathrm{a}$ & 3041.04 & 0.57840 & YES & YES \\
\hline 78 & $\mathrm{a}$ & 3046.35 & 10.72934 & YES & YES \\
\hline 79 & $\mathrm{a}$ & 3060.15 & 4.60325 & YES & YES \\
\hline 80 & $\mathrm{a}$ & 3079.46 & 0.08100 & YES & YES \\
\hline 81 & $\mathrm{a}$ & 3083.02 & 0.11941 & YES & YES \\
\hline 82 & $\mathrm{a}$ & 3103.46 & 0.07773 & YES & YES \\
\hline 83 & $\mathrm{a}$ & 3200.61 & 13.96766 & YES & YES \\
\hline 84 & $\mathrm{a}$ & 3217.38 & 16.41182 & YES & YES \\
\hline
\end{tabular}




\begin{tabular}{|c|c|c|c|c|c|}
\hline $\begin{array}{l}{[\mathbf{B P y}]^{+}} \\
\# \text { mode }\end{array}$ & & netry & number & intensity & 1 \\
\hline \# & & $\mathrm{cm}^{* *}(-1)$ & $\mathrm{km} / \mathrm{mol}$ & IR & RAMAN \\
\hline 1 & & 0.00 & 0.00000 & - $\quad-$ & \\
\hline 2 & & 0.00 & 0.00000 & - & \\
\hline 3 & & 0.00 & 0.00000 & - & \\
\hline 4 & & 0.00 & 0.00000 & - & \\
\hline 5 & & 0.00 & 0.00000 & - & \\
\hline 6 & & 0.00 & 0.00000 & $-\quad-$ & \\
\hline 7 & $\mathrm{a}$ & 37.43 & 0.26031 & YES & YES \\
\hline 8 & $\mathrm{a}$ & 75.16 & 0.13368 & YES & YES \\
\hline 9 & $\mathrm{a}$ & 75.57 & 0.39310 & YES & YES \\
\hline 10 & $\mathrm{a}$ & 108.79 & 0.10796 & YES & YES \\
\hline 11 & $\mathrm{a}$ & 200.50 & 0.34457 & YES & YES \\
\hline 12 & $\mathrm{a}$ & 244.28 & 0.01383 & YES & YES \\
\hline 13 & $\mathrm{a}$ & 279.20 & 0.34533 & YES & YES \\
\hline 14 & $\mathrm{a}$ & 375.58 & 0.09978 & YES & YES \\
\hline 15 & $\mathrm{a}$ & 382.25 & 0.02904 & YES & YES \\
\hline 16 & $\mathrm{a}$ & 393.80 & 0.01298 & YES & YES \\
\hline 17 & $\mathrm{a}$ & 492.24 & 8.11927 & YES & YES \\
\hline 18 & $\mathrm{a}$ & 579.35 & 2.10133 & YES & YES \\
\hline 19 & $\mathrm{a}$ & 639.09 & 0.03966 & YES & YES \\
\hline 20 & $\mathrm{a}$ & 681.02 & 45.80440 & YES & YES \\
\hline 21 & $\mathrm{a}$ & 732.45 & 3.98397 & YES & YES \\
\hline 22 & $\mathrm{a}$ & 763.29 & 26.20612 & YES & YES \\
\hline 23 & $\mathrm{a}$ & 782.73 & 0.48906 & YES & YES \\
\hline 24 & $\mathrm{a}$ & 797.12 & 1.68882 & YES & YES \\
\hline 25 & $\mathrm{a}$ & 841.67 & 0.01328 & YES & YES \\
\hline 26 & $\mathrm{a}$ & 897.23 & 0.53559 & YES & YES \\
\hline 27 & $\mathrm{a}$ & 921.62 & 0.27571 & YES & YES \\
\hline 28 & $\mathrm{a}$ & 934.66 & 0.58237 & YES & YES \\
\hline 29 & $\mathrm{a}$ & 967.34 & 0.01899 & YES & YES \\
\hline 30 & $\mathrm{a}$ & 1004.49 & 1.19097 & YES & YES \\
\hline 31 & $\mathrm{a}$ & 1010.19 & 0.12528 & YES & YES \\
\hline 32 & $\mathrm{a}$ & 1019.34 & 2.91925 & YES & YES \\
\hline 33 & $\mathrm{a}$ & 1044.73 & 0.51172 & YES & YES \\
\hline 34 & $\mathrm{a}$ & 1046.81 & 1.41636 & YES & YES \\
\hline 35 & $\mathrm{a}$ & 1071.39 & 3.89920 & YES & YES \\
\hline 36 & $\mathrm{a}$ & 1098.06 & 14.79470 & YES & YES \\
\hline 37 & $\mathrm{a}$ & 1136.50 & 1.30555 & YES & YES \\
\hline 38 & $\mathrm{a}$ & 1149.87 & 0.30464 & YES & YES \\
\hline 39 & $\mathrm{a}$ & 1151.91 & 31.95232 & YES & YES \\
\hline 40 & $\mathrm{a}$ & 1194.14 & 2.95935 & YES & YES \\
\hline 41 & $\mathrm{a}$ & 1202.96 & 0.33989 & YES & YES \\
\hline 42 & $\mathrm{a}$ & 1250.85 & 3.21025 & YES & YES \\
\hline 43 & $\mathrm{a}$ & 1268.51 & 0.33300 & YES & YES \\
\hline 44 & $\mathrm{a}$ & 1292.18 & 3.79179 & YES & YES \\
\hline 45 & $\mathrm{a}$ & 1329.52 & 0.41845 & YES & YES \\
\hline 46 & $\mathrm{a}$ & 1336.97 & 2.21144 & YES & YES \\
\hline
\end{tabular}




$\begin{array}{lllccc}47 & \mathrm{a} & 1351.49 & 1.15932 & \text { YES } & \text { YES } \\ 48 & \mathrm{a} & 1367.17 & 2.75146 & \text { YES } & \text { YES } \\ 49 & \mathrm{a} & 1373.24 & 1.41086 & \text { YES } & \text { YES } \\ 50 & \mathrm{a} & 1426.73 & 4.87043 & \text { YES } & \text { YES } \\ 51 & \mathrm{a} & 1432.75 & 2.33428 & \text { YES } & \text { YES } \\ 52 & \mathrm{a} & 1438.91 & 14.38905 & \text { YES } & \text { YES } \\ 53 & \mathrm{a} & 1441.94 & 2.73461 & \text { YES } & \text { YES } \\ 54 & \mathrm{a} & 1452.27 & 16.07309 & \text { YES } & \text { YES } \\ 55 & \mathrm{a} & 1477.61 & 44.19980 & \text { YES } & \text { YES } \\ 56 & \mathrm{a} & 1487.43 & 12.51477 & \text { YES } & \text { YES } \\ 57 & \mathrm{a} & 1588.74 & 0.64454 & \text { YES } & \text { YES } \\ 58 & \mathrm{a} & 1637.46 & 25.29503 & \text { YES } & \text { YES } \\ 59 & \mathrm{a} & 2937.93 & 1.19739 & \text { YES } & \text { YES } \\ 60 & \mathrm{a} & 2946.47 & 19.48271 & \text { YES } & \text { YES } \\ 61 & \mathrm{a} & 2951.81 & 28.39298 & \text { YES } & \text { YES } \\ 62 & \mathrm{a} & 2979.28 & 0.52135 & \text { YES } & \text { YES } \\ 63 & \mathrm{a} & 3000.30 & 14.34190 & \text { YES } & \text { YES } \\ 64 & \mathrm{a} & 3000.77 & 5.79318 & \text { YES } & \text { YES } \\ 65 & \mathrm{a} & 3035.33 & 22.57436 & \text { YES } & \text { YES } \\ 66 & \mathrm{a} & 3048.11 & 7.94764 & \text { YES } & \text { YES } \\ 67 & \mathrm{a} & 3061.95 & 2.30285 & \text { YES } & \text { YES } \\ 68 & \mathrm{a} & 3132.23 & 0.07801 & \text { YES } & \text { YES } \\ 69 & \mathrm{a} & 3141.66 & 0.63916 & \text { YES } & \text { YES } \\ 70 & \mathrm{a} & 3144.77 & 8.25125 & \text { YES } & \text { YES } \\ 71 & \mathrm{a} & 3150.88 & 11.99534 & \text { YES } & \text { YES } \\ 72 & \mathrm{a} & 3154.88 & 2.71158 & \text { YES } & \text { YES }\end{array}$

\section{[BMPyr $^{+}$}

\# mode symmetry wave number IR intensity selection rules

\#

$\begin{array}{ccccccc} & & \mathrm{cm} * *(-1) & \mathrm{km} / \mathrm{mol} & \mathrm{IR} & \text { RAMAN } \\ 1 & & 0.00 & 0.00000 & - & - & \\ 2 & & 0.00 & 0.00000 & - & - & \\ 3 & & 0.00 & 0.00000 & - & - & \\ 4 & & 0.00 & 0.00000 & - & - & \\ 5 & & 0.00 & 0.00000 & - & - & \\ 6 & & 0.00 & 0.00000 & - & - & \\ 7 & \mathrm{a} & 53.47 & 0.15571 & \text { YES } & \text { YES } \\ 8 & \mathrm{a} & 70.16 & 0.37552 & \text { YES } & \text { YES } \\ 9 & \mathrm{a} & 100.27 & 0.09596 & \text { YES } & \text { YES } \\ 10 & \mathrm{a} & 108.24 & 0.30067 & \text { YES } & \text { YES } \\ 11 & \mathrm{a} & 135.54 & 0.29921 & \text { YES } & \text { YES } \\ 12 & \mathrm{a} & 193.87 & 0.37866 & \text { YES } & \text { YES } \\ 13 & \mathrm{a} & 233.46 & 0.34989 & \text { YES } & \text { YES } \\ 14 & \mathrm{a} & 247.52 & 0.01730 & \text { YES } & \text { YES } \\ 15 & \mathrm{a} & 274.33 & 0.36390 & \text { YES } & \text { YES } \\ 16 & \mathrm{a} & 286.36 & 0.29292 & \text { YES } & \text { YES } \\ 17 & \mathrm{a} & 358.29 & 0.25218 & \text { YES } & \text { YES } \\ 18 & \mathrm{a} & 385.32 & 0.13328 & \text { YES } & \text { YES } \\ 19 & \mathrm{a} & 431.12 & 0.35150 & \text { YES } & \text { YES }\end{array}$


S-38

\begin{tabular}{|c|c|c|c|c|c|}
\hline 20 & $\mathrm{a}$ & 460.54 & 0.73451 & YES & YES \\
\hline 21 & $\mathrm{a}$ & 620.76 & 1.67414 & YES & YES \\
\hline 22 & $\mathrm{a}$ & 636.81 & 0.34210 & YES & YES \\
\hline 23 & $\mathrm{a}$ & 715.42 & 2.85575 & YES & YES \\
\hline 24 & $\mathrm{a}$ & 734.98 & 5.75267 & YES & YES \\
\hline 25 & $\mathrm{a}$ & 785.38 & 1.34809 & YES & YES \\
\hline 26 & $\mathrm{a}$ & 797.04 & 2.53725 & YES & YES \\
\hline 27 & $\mathrm{a}$ & 802.28 & 6.77193 & YES & YES \\
\hline 28 & $\mathrm{a}$ & 890.15 & 1.80116 & YES & YES \\
\hline 29 & $\mathrm{a}$ & 896.08 & 1.05499 & YES & YES \\
\hline 30 & $\mathrm{a}$ & 899.93 & 46.14915 & YES & YES \\
\hline 31 & $\mathrm{a}$ & 908.32 & 6.91418 & YES & YES \\
\hline 32 & $\mathrm{a}$ & 936.48 & 1.15684 & YES & YES \\
\hline 33 & $\mathrm{a}$ & 943.90 & 5.01018 & YES & YES \\
\hline 34 & $\mathrm{a}$ & 987.04 & 8.07012 & YES & YES \\
\hline 35 & $\mathrm{a}$ & 1003.37 & 3.37802 & YES & YES \\
\hline 36 & $\mathrm{a}$ & 1026.19 & 9.41068 & YES & YES \\
\hline 37 & $\mathrm{a}$ & 1048.93 & 4.96174 & YES & YES \\
\hline 38 & $\mathrm{a}$ & 1053.07 & 0.40430 & YES & YES \\
\hline 39 & $\mathrm{a}$ & 1056.08 & 0.28838 & YES & YES \\
\hline 40 & $\mathrm{a}$ & 1105.20 & 5.50822 & YES & YES \\
\hline 41 & $\mathrm{a}$ & 1119.15 & 0.58289 & YES & YES \\
\hline 42 & $\mathrm{a}$ & 1152.40 & 0.06330 & YES & YES \\
\hline 43 & $\mathrm{a}$ & 1174.66 & 0.42523 & YES & YES \\
\hline 44 & $\mathrm{a}$ & 1208.43 & 1.90110 & YES & YES \\
\hline 45 & $\mathrm{a}$ & 1215.77 & 0.22662 & YES & YES \\
\hline 46 & $\mathrm{a}$ & 1221.28 & 2.04742 & YES & YES \\
\hline 47 & $\mathrm{a}$ & 1233.05 & 4.43175 & YES & YES \\
\hline 48 & $\mathrm{a}$ & 1256.65 & 1.60706 & YES & YES \\
\hline 49 & $\mathrm{a}$ & 1265.60 & 2.08127 & YES & YES \\
\hline 50 & $\mathrm{a}$ & 1286.01 & 0.05844 & YES & YES \\
\hline 51 & $\mathrm{a}$ & 1292.78 & 1.58382 & YES & YES \\
\hline 52 & $\mathrm{a}$ & 1301.84 & 3.14809 & YES & YES \\
\hline 53 & $\mathrm{a}$ & 1318.73 & 1.56346 & YES & YES \\
\hline 54 & $\mathrm{a}$ & 1324.15 & 1.82534 & YES & YES \\
\hline 55 & $\mathrm{a}$ & 1344.07 & 2.87792 & YES & YES \\
\hline 56 & $\mathrm{a}$ & 1351.01 & 12.31775 & YES & YES \\
\hline 57 & $\mathrm{a}$ & 1373.89 & 6.17098 & YES & YES \\
\hline 58 & $\mathrm{a}$ & 1378.44 & 5.51292 & YES & YES \\
\hline 59 & $\mathrm{a}$ & 1400.30 & 12.73678 & YES & YES \\
\hline 60 & $\mathrm{a}$ & 1420.04 & 2.50568 & YES & YES \\
\hline 61 & $\mathrm{a}$ & 1428.93 & 1.03432 & YES & YES \\
\hline 62 & $\mathrm{a}$ & 1431.88 & 1.18751 & YES & YES \\
\hline 63 & $\mathrm{a}$ & 1434.67 & 5.88040 & YES & YES \\
\hline 64 & $\mathrm{a}$ & 1437.70 & 14.63024 & YES & YES \\
\hline 65 & $\mathrm{a}$ & 1440.45 & 22.92449 & YES & YES \\
\hline 66 & $\mathrm{a}$ & 1441.93 & 13.86690 & YES & YES \\
\hline 67 & $\mathrm{a}$ & 1448.64 & 2.28699 & YES & YES \\
\hline 68 & $\mathrm{a}$ & 1449.52 & 9.55634 & YES & YES \\
\hline
\end{tabular}




$\begin{array}{llcccc}69 & \mathrm{a} & 1457.40 & 43.86592 & \text { YES } & \text { YES } \\ 70 & \mathrm{a} & 1468.82 & 3.44013 & \text { YES } & \text { YES } \\ 71 & \mathrm{a} & 2939.70 & 7.41425 & \text { YES } & \text { YES } \\ 72 & \mathrm{a} & 2949.90 & 11.96197 & \text { YES } & \text { YES } \\ 73 & \mathrm{a} & 2954.02 & 25.69242 & \text { YES } & \text { YES } \\ 74 & \mathrm{a} & 2979.55 & 8.06196 & \text { YES } & \text { YES } \\ 75 & \mathrm{a} & 2981.64 & 3.20714 & \text { YES } & \text { YES } \\ 76 & \mathrm{a} & 2998.29 & 0.39772 & \text { YES } & \text { YES } \\ 77 & \mathrm{a} & 3000.78 & 4.24674 & \text { YES } & \text { YES } \\ 78 & \mathrm{a} & 3005.02 & 2.66187 & \text { YES } & \text { YES } \\ 79 & \mathrm{a} & 3005.91 & 4.72836 & \text { YES } & \text { YES } \\ 80 & \mathrm{a} & 3007.76 & 7.26823 & \text { YES } & \text { YES } \\ 81 & \mathrm{a} & 3009.83 & 5.77134 & \text { YES } & \text { YES } \\ 82 & \mathrm{a} & 3033.77 & 20.88470 & \text { YES } & \text { YES } \\ 83 & \mathrm{a} & 3041.97 & 11.35402 & \text { YES } & \text { YES } \\ 84 & \mathrm{a} & 3048.09 & 0.17443 & \text { YES } & \text { YES } \\ 85 & \mathrm{a} & 3048.87 & 8.98482 & \text { YES } & \text { YES } \\ 86 & \mathrm{a} & 3059.72 & 1.04902 & \text { YES } & \text { YES } \\ 87 & \mathrm{a} & 3074.36 & 1.15911 & \text { YES } & \text { YES } \\ 88 & \mathrm{a} & 3082.96 & 0.82340 & \text { YES } & \text { YES } \\ 89 & \mathrm{a} & 3102.59 & 0.17155 & \text { YES } & \text { YES } \\ 90 & \mathrm{a} & 3113.33 & 0.19077 & \text { YES } & \text { YES }\end{array}$

\section{[C $\left._{5} \mathrm{MPyr}\right]^{+}$}

\# mode symmetry wave number IR intensity selection rules \#

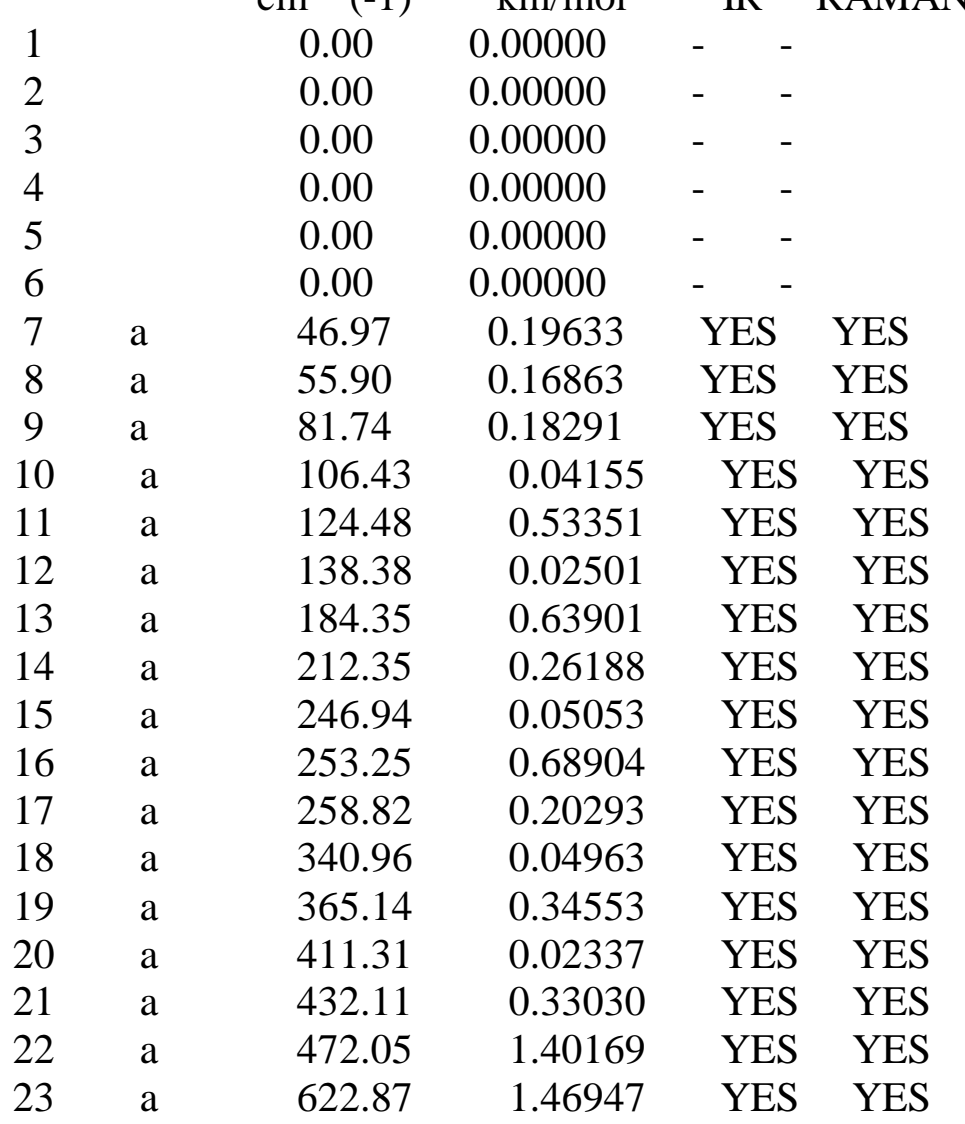




\begin{tabular}{|c|c|c|c|c|c|}
\hline 24 & $\mathrm{a}$ & 637.91 & 0.44662 & YES & YES \\
\hline 25 & $\mathrm{a}$ & 715.44 & 3.02158 & YES & YES \\
\hline 26 & $\mathrm{a}$ & 730.97 & 5.67982 & YES & YES \\
\hline 27 & $\mathrm{a}$ & 755.45 & 0.41955 & YES & YES \\
\hline 28 & $\mathrm{a}$ & 795.78 & 4.37616 & YES & YES \\
\hline 29 & $\mathrm{a}$ & 802.33 & 6.36019 & YES & YES \\
\hline 30 & $\mathrm{a}$ & 835.70 & 3.34138 & YES & YES \\
\hline 31 & $\mathrm{a}$ & 885.79 & 10.81095 & YES & YES \\
\hline 32 & a & 891.98 & 0.59475 & YES & YES \\
\hline 33 & $\mathrm{a}$ & 905.27 & 34.55178 & YES & YES \\
\hline 34 & $\mathrm{a}$ & 914.68 & 3.52885 & YES & YES \\
\hline 35 & $\mathrm{a}$ & 941.65 & 11.22310 & YES & YES \\
\hline 36 & $\mathrm{a}$ & 960.85 & 2.68837 & YES & YES \\
\hline 37 & $\mathrm{a}$ & 987.30 & 8.21694 & YES & YES \\
\hline 38 & $\mathrm{a}$ & 1000.68 & 3.64446 & YES & YES \\
\hline 39 & $\mathrm{a}$ & 1017.10 & 1.60552 & YES & YES \\
\hline 40 & $\mathrm{a}$ & 1039.44 & 12.67153 & YES & YES \\
\hline 41 & $\mathrm{a}$ & 1051.76 & 1.33973 & YES & YES \\
\hline 42 & $\mathrm{a}$ & 1054.54 & 0.43590 & YES & YES \\
\hline 43 & $\mathrm{a}$ & 1059.68 & 2.00243 & YES & YES \\
\hline 44 & $\mathrm{a}$ & 1108.63 & 3.97783 & YES & YES \\
\hline 45 & $\mathrm{a}$ & 1121.45 & 0.14984 & YES & YES \\
\hline 46 & $\mathrm{a}$ & 1152.33 & 0.20277 & YES & YES \\
\hline 47 & $\mathrm{a}$ & 1174.36 & 0.25142 & YES & YES \\
\hline 48 & $\mathrm{a}$ & 1201.21 & 0.53977 & YES & YES \\
\hline 49 & $\mathrm{a}$ & 1215.30 & 0.58160 & YES & YES \\
\hline 50 & $\mathrm{a}$ & 1218.75 & 1.86112 & YES & YES \\
\hline 51 & $\mathrm{a}$ & 1226.34 & 2.31218 & YES & YES \\
\hline 52 & $\mathrm{a}$ & 1241.93 & 2.82485 & YES & YES \\
\hline 53 & $\mathrm{a}$ & 1248.35 & 2.67546 & YES & YES \\
\hline 54 & $\mathrm{a}$ & 1280.59 & 1.81710 & YES & YES \\
\hline 55 & $\mathrm{a}$ & 1286.49 & 0.01916 & YES & YES \\
\hline 56 & $\mathrm{a}$ & 1296.27 & 2.07944 & YES & YES \\
\hline 57 & $\mathrm{a}$ & 1296.95 & 2.76824 & YES & YES \\
\hline 58 & $\mathrm{a}$ & 1308.14 & 1.84047 & YES & YES \\
\hline 59 & $\mathrm{a}$ & 1318.74 & 1.70116 & YES & YES \\
\hline 60 & $\mathrm{a}$ & 1328.95 & 6.77252 & YES & YES \\
\hline 61 & $\mathrm{a}$ & 1346.78 & 8.52571 & YES & YES \\
\hline 62 & $\mathrm{a}$ & 1361.15 & 0.15703 & YES & YES \\
\hline 63 & $\mathrm{a}$ & 1370.24 & 2.95582 & YES & YES \\
\hline 64 & $\mathrm{a}$ & 1377.16 & 8.39985 & YES & YES \\
\hline 65 & $\mathrm{a}$ & 1404.89 & 12.52420 & YES & YES \\
\hline 66 & $\mathrm{a}$ & 1420.22 & 2.59207 & YES & YES \\
\hline 67 & $\mathrm{a}$ & 1429.92 & 3.02288 & YES & YES \\
\hline 68 & $\mathrm{a}$ & 1430.40 & 1.07756 & YES & YES \\
\hline 69 & $\mathrm{a}$ & 1432.46 & 1.79779 & YES & YES \\
\hline 70 & $\mathrm{a}$ & 1436.76 & 8.34841 & YES & YES \\
\hline 71 & $\mathrm{a}$ & 1440.32 & 11.84627 & YES & YES \\
\hline 72 & $\mathrm{a}$ & 1440.70 & 27.07195 & YES & YES \\
\hline
\end{tabular}




$\begin{array}{llcccc}73 & \mathrm{a} & 1444.09 & 1.96489 & \text { YES } & \text { YES } \\ 74 & \mathrm{a} & 1449.63 & 2.08093 & \text { YES } & \text { YES } \\ 75 & \mathrm{a} & 1451.56 & 14.28802 & \text { YES } & \text { YES } \\ 76 & \mathrm{a} & 1458.17 & 45.28337 & \text { YES } & \text { YES } \\ 77 & \mathrm{a} & 1469.85 & 2.97834 & \text { YES } & \text { YES } \\ 78 & \mathrm{a} & 2927.51 & 0.91625 & \text { YES } & \text { YES } \\ 79 & \mathrm{a} & 2936.32 & 26.85862 & \text { YES } & \text { YES } \\ 80 & \mathrm{a} & 2946.63 & 23.46821 & \text { YES } & \text { YES } \\ 81 & \mathrm{a} & 2952.58 & 24.46941 & \text { YES } & \text { YES } \\ 82 & \mathrm{a} & 2965.78 & 0.03141 & \text { YES } & \text { YES } \\ 83 & \mathrm{a} & 2978.96 & 8.22145 & \text { YES } & \text { YES } \\ 84 & \mathrm{a} & 2985.83 & 15.15940 & \text { YES } & \text { YES } \\ 85 & \mathrm{a} & 2998.13 & 0.50676 & \text { YES } & \text { YES } \\ 86 & \mathrm{a} & 3000.56 & 4.27245 & \text { YES } & \text { YES } \\ 87 & \mathrm{a} & 3004.69 & 2.33408 & \text { YES } & \text { YES } \\ 88 & \mathrm{a} & 3005.73 & 5.56385 & \text { YES } & \text { YES } \\ 89 & \mathrm{a} & 3007.67 & 11.85407 & \text { YES } & \text { YES } \\ 90 & \mathrm{a} & 3009.41 & 7.81357 & \text { YES } & \text { YES } \\ 91 & \mathrm{a} & 3028.98 & 28.99535 & \text { YES } & \text { YES } \\ 92 & \mathrm{a} & 3041.16 & 13.18459 & \text { YES } & \text { YES } \\ 93 & \mathrm{a} & 3041.30 & 9.73348 & \text { YES } & \text { YES } \\ 94 & \mathrm{a} & 3048.08 & 0.18619 & \text { YES } & \text { YES } \\ 95 & \mathrm{a} & 3059.63 & 1.01637 & \text { YES } & \text { YES } \\ 96 & \mathrm{a} & 3073.79 & 1.15738 & \text { YES } & \text { YES } \\ 97 & \mathrm{a} & 3082.34 & 0.86785 & \text { YES } & \text { YES } \\ 98 & \mathrm{a} & 3102.10 & 0.19325 & \text { YES } & \text { YES } \\ 99 & \mathrm{a} & 3113.44 & 0.24956 & \text { YES } & \text { YES }\end{array}$

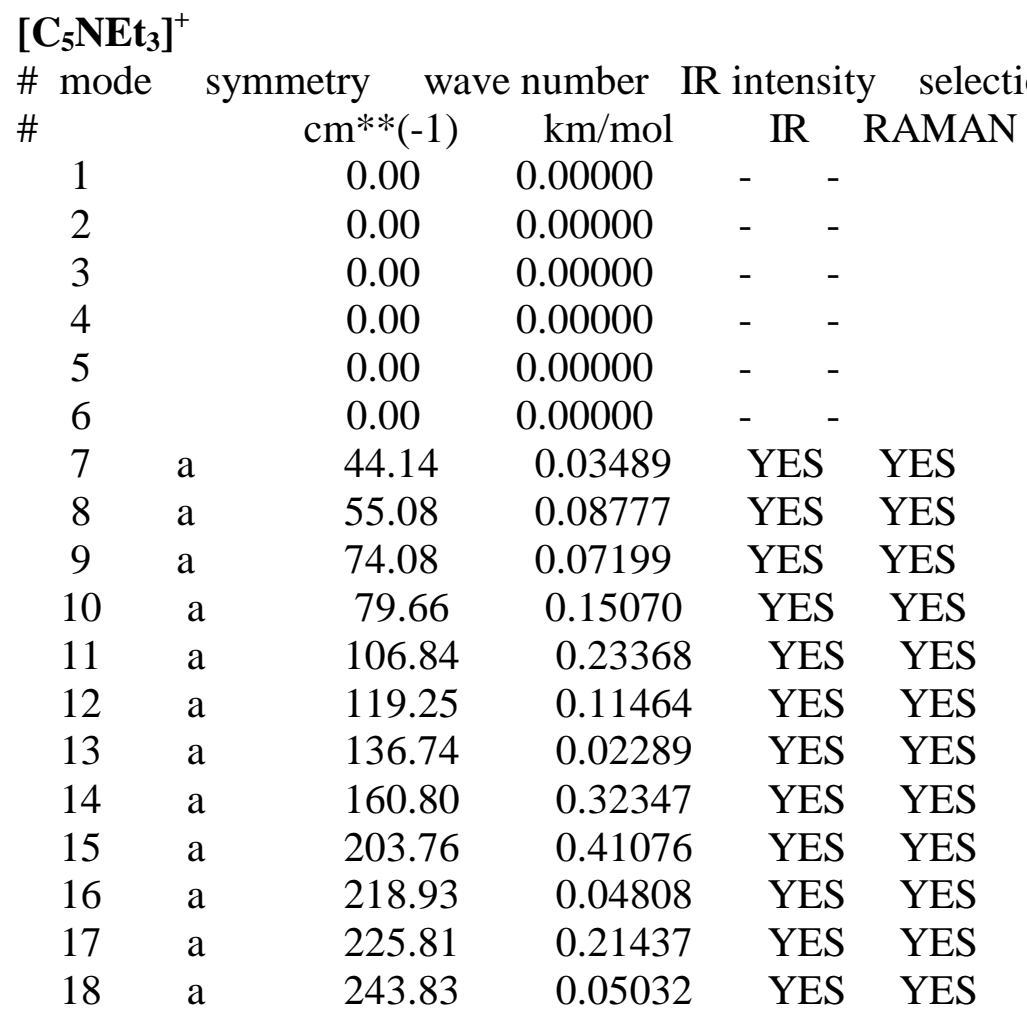




\begin{tabular}{|c|c|c|c|c|c|}
\hline 19 & $\mathrm{a}$ & 248.62 & 0.00906 & YES & YES \\
\hline 20 & $\mathrm{a}$ & 277.12 & 0.15401 & YES & YES \\
\hline 21 & $\mathrm{a}$ & 281.88 & 0.22026 & YES & YES \\
\hline 22 & $\mathrm{a}$ & 331.01 & 0.02877 & YES & YES \\
\hline 23 & $\mathrm{a}$ & 343.98 & 0.10459 & YES & YES \\
\hline 24 & $\mathrm{a}$ & 370.41 & 0.40146 & YES & YES \\
\hline 25 & $\mathrm{a}$ & 376.28 & 0.32350 & YES & YES \\
\hline 26 & $\mathrm{a}$ & 435.73 & 1.12025 & YES & YES \\
\hline 27 & $\mathrm{a}$ & 452.14 & 1.39941 & YES & YES \\
\hline 28 & $\mathrm{a}$ & 525.45 & 2.67171 & YES & YES \\
\hline 29 & $\mathrm{a}$ & 534.28 & 2.24321 & YES & YES \\
\hline 30 & $\mathrm{a}$ & 671.91 & 1.04558 & YES & YES \\
\hline 31 & a & 730.08 & 6.39323 & YES & YES \\
\hline 32 & a & 751.35 & 6.81223 & YES & YES \\
\hline 33 & a & 768.78 & 8.68271 & YES & YES \\
\hline 34 & $\mathrm{a}$ & 787.67 & 15.75955 & YES & YES \\
\hline 35 & $\mathrm{a}$ & 794.15 & 6.26524 & YES & YES \\
\hline 36 & a & 822.37 & 2.56258 & YES & YES \\
\hline 37 & $\mathrm{a}$ & 864.22 & 4.45331 & YES & YES \\
\hline 38 & $\mathrm{a}$ & 871.07 & 1.92747 & YES & YES \\
\hline 39 & $\mathrm{a}$ & 882.96 & 1.59803 & YES & YES \\
\hline 40 & a & 915.13 & 12.53633 & YES & YES \\
\hline 41 & a & 966.36 & 1.02718 & YES & YES \\
\hline 42 & $\mathrm{a}$ & 991.72 & 24.01678 & YES & YES \\
\hline 43 & $\mathrm{a}$ & 1004.22 & 18.93923 & YES & YES \\
\hline 44 & $\mathrm{a}$ & 1009.41 & 6.48482 & YES & YES \\
\hline 45 & a & 1024.61 & 15.46447 & YES & YES \\
\hline 46 & $\mathrm{a}$ & 1031.74 & 11.38713 & YES & YES \\
\hline 47 & $\mathrm{a}$ & 1053.65 & 2.76127 & YES & YES \\
\hline 48 & a & 1061.27 & 0.22870 & YES & YES \\
\hline 49 & a & 1077.69 & 7.47405 & YES & YES \\
\hline 50 & $\mathrm{a}$ & 1088.00 & 4.18886 & YES & YES \\
\hline 51 & $\mathrm{a}$ & 1122.69 & 2.03387 & YES & YES \\
\hline 52 & $\mathrm{a}$ & 1123.06 & 2.59889 & YES & YES \\
\hline 53 & a & 1147.63 & 10.51775 & YES & YES \\
\hline 54 & $\mathrm{a}$ & 1166.92 & 6.34792 & YES & YES \\
\hline 55 & $\mathrm{a}$ & 1172.35 & 3.43271 & YES & YES \\
\hline 56 & a & 1213.38 & 1.69869 & YES & YES \\
\hline 57 & $\mathrm{a}$ & 1237.93 & 1.51669 & YES & YES \\
\hline 58 & a & 1275.05 & 0.20747 & YES & YES \\
\hline 59 & $\mathrm{a}$ & 1281.19 & 2.48239 & YES & YES \\
\hline 60 & $\mathrm{a}$ & 1294.56 & 5.34318 & YES & YES \\
\hline 61 & a & 1296.21 & 0.25200 & YES & YES \\
\hline 62 & $\mathrm{a}$ & 1310.46 & 4.66554 & YES & YES \\
\hline 63 & $\mathrm{a}$ & 1320.04 & 9.10967 & YES & YES \\
\hline 64 & $\mathrm{a}$ & 1322.25 & 5.25390 & YES & YES \\
\hline 65 & $\mathrm{a}$ & 1345.97 & 1.36977 & YES & YES \\
\hline 66 & a & 1357.71 & 0.70709 & YES & YES \\
\hline 67 & $\mathrm{a}$ & 1358.25 & 1.73602 & YES & YES \\
\hline
\end{tabular}


S-43

\begin{tabular}{|c|c|c|c|c|c|}
\hline 68 & $\mathrm{a}$ & 1361.71 & 4.78850 & YES & YES \\
\hline 69 & $\mathrm{a}$ & 1372.53 & 5.30946 & YES & YES \\
\hline 70 & $\mathrm{a}$ & 1375.88 & 18.94480 & YES & YES \\
\hline 71 & $\mathrm{a}$ & 1378.01 & 1.33445 & YES & YES \\
\hline 72 & $\mathrm{a}$ & 1387.88 & 20.34353 & YES & YES \\
\hline 73 & $\mathrm{a}$ & 1392.25 & 13.30715 & YES & YES \\
\hline 74 & $\mathrm{a}$ & 1420.52 & 0.71523 & YES & YES \\
\hline 75 & $\mathrm{a}$ & 1426.19 & 19.49408 & YES & YES \\
\hline 76 & $\mathrm{a}$ & 1427.60 & 2.08801 & YES & YES \\
\hline 77 & $\mathrm{a}$ & 1432.64 & 8.00363 & YES & YES \\
\hline 78 & $\mathrm{a}$ & 1434.64 & 13.35464 & YES & YES \\
\hline 79 & $\mathrm{a}$ & 1440.07 & 10.18246 & YES & YES \\
\hline 80 & $\mathrm{a}$ & 1440.65 & 8.10327 & YES & YES \\
\hline 81 & $\mathrm{a}$ & 1440.74 & 11.46256 & YES & YES \\
\hline 82 & $\mathrm{a}$ & 1442.70 & 6.13425 & YES & YES \\
\hline 83 & $\mathrm{a}$ & 1445.96 & 5.21616 & YES & YES \\
\hline 84 & $\mathrm{a}$ & 1447.01 & 11.17822 & YES & YES \\
\hline 85 & $\mathrm{a}$ & 1447.05 & 8.17067 & YES & YES \\
\hline 86 & $\mathrm{a}$ & 1450.80 & 19.07007 & YES & YES \\
\hline 87 & $\mathrm{a}$ & 1455.58 & 6.43736 & YES & YES \\
\hline 88 & $\mathrm{a}$ & 1461.44 & 8.92071 & YES & YES \\
\hline 89 & $\mathrm{a}$ & 2924.65 & 2.89930 & YES & YES \\
\hline 90 & $\mathrm{a}$ & 2935.45 & 31.13381 & YES & YES \\
\hline 91 & $\mathrm{a}$ & 2946.70 & 26.83686 & YES & YES \\
\hline 92 & $\mathrm{a}$ & 2963.32 & 0.35915 & YES & YES \\
\hline 93 & $\mathrm{a}$ & 2971.61 & 2.07623 & YES & YES \\
\hline 94 & $\mathrm{a}$ & 2972.42 & 7.39273 & YES & YES \\
\hline 95 & $\mathrm{a}$ & 2972.98 & 0.78494 & YES & YES \\
\hline 96 & $\mathrm{a}$ & 2975.10 & 5.38436 & YES & YES \\
\hline 97 & $\mathrm{a}$ & 2985.39 & 22.08456 & YES & YES \\
\hline 98 & $\mathrm{a}$ & 2988.40 & 12.76425 & YES & YES \\
\hline 99 & $\mathrm{a}$ & 2997.74 & 4.32411 & YES & YES \\
\hline 100 & $\mathrm{a}$ & 3003.85 & 5.18557 & YES & YES \\
\hline 101 & $\mathrm{a}$ & 3011.16 & 4.41791 & YES & YES \\
\hline 102 & $\mathrm{a}$ & 3028.29 & 19.54638 & YES & YES \\
\hline 103 & $\mathrm{a}$ & 3030.81 & 17.40162 & YES & YES \\
\hline 104 & $\mathrm{a}$ & 3042.21 & 13.51169 & YES & YES \\
\hline 105 & $\mathrm{a}$ & 3052.01 & 4.04600 & YES & YES \\
\hline 106 & $\mathrm{a}$ & 3052.72 & 0.67673 & YES & YES \\
\hline 107 & $\mathrm{a}$ & 3053.06 & 0.45681 & YES & YES \\
\hline 108 & $\mathrm{a}$ & 3054.64 & 3.93902 & YES & YES \\
\hline 109 & $\mathrm{a}$ & 3058.70 & 5.90819 & YES & YES \\
\hline 110 & $\mathrm{a}$ & 3064.00 & 1.79450 & YES & YES \\
\hline 111 & $\mathrm{a}$ & 3065.47 & 1.06164 & YES & YES \\
\hline 112 & $\mathrm{a}$ & 3078.47 & 8.05911 & YES & YES \\
\hline 113 & $\mathrm{a}$ & 3083.14 & 9.09476 & YES & YES \\
\hline 114 & $\mathrm{a}$ & 3086.40 & 3.71205 & YES & YES \\
\hline
\end{tabular}




\section{Details of volume determinations for each ion:}

Part 1 - From X-ray structures:

\section{Anions:}

$\left[\mathrm{BF}_{4}\right]^{-}$

$0.073 \pm 0.009 \mathrm{~nm}^{3}$ (5 salts considered)

Volume taken from Jenkins et al., Inorg Chem, 1999, 38, 3609.

$\left[\mathrm{PF}_{6}\right]^{-}$

$0.109 \pm 0.008 \mathrm{~nm}^{3}$ (4 salts considered)

Volume taken from Jenkins et al., Inorg Chem, 1999, 38, 3609.

\section{$[\mathrm{TfO}]^{-}$}

\section{$0.131 \pm 0.015 \mathrm{~nm}^{3}$ (2 salts considered)}

1) $\left[\mathrm{NH}_{4}\right][\mathrm{TfO}]$

$V_{\text {cell }}\left(\mathrm{A}^{+} \mathrm{X}^{-}\right)=311.6 \AA^{3}$

$Z=2$

$\underline{\text { Volume }=0.135 \mathrm{~nm}^{3}}$

$\left[\mathrm{NH}_{4}\right]^{+}$volume $=0.021 \pm 0.015 \mathrm{~nm}^{3}$ (3 salts considered), volume taken from Jenkins et al. Inorg Chem, 1999, 38, 3609.

2) $\left[\mathrm{NH}_{4}\right][\mathrm{TfO}]$ (same space group as above, but different cell dimensions)

$V_{\text {cell }}\left(\mathrm{A}^{+} \mathrm{X}^{-}\right)=604.1 \AA^{3}$

$Z=4$

$\underline{\text { Volume }=0.130 \mathrm{~nm}^{3}}$ 
$\left[\mathrm{NH}_{4}\right]^{+}$volume $=0.021 \pm 0.015 \mathrm{~nm}^{3}$ (3 salts considered), volume taken from Jenkins et al.

Inorg Chem, 1999, 38, 3609.

3) $[\mathrm{Li}][\mathrm{TfO}]$

$V_{\text {cell }}\left(\mathrm{A}^{+} \mathrm{X}^{-}\right)=485.3 \AA^{3}$

$Z=4$

$\underline{\text { Volume }=0.119 \mathrm{~nm}^{3}}$

$[\mathrm{Li}]^{+}$volume $=0.00199 \mathrm{~nm}^{3}$, volume taken from Jenkins et al., Inorg Chem, 1999, 38, 3609.

4) $[\mathrm{Na}][\mathrm{TfO}]$

$V_{\text {cell }}\left(\mathrm{A}^{+} \mathrm{X}^{-}\right)=1069.6 \AA^{3}$

$Z=8$ (tetrameric asymmetric unit)

$\underline{\text { Volume }=0.130 \mathrm{~nm}^{3}}$

$[\mathrm{Na}]^{+}$volume $=0.00394$, volume taken from Jenkins et al., Inorg Chem, 1999, 38, 3609.

5) $[\mathrm{K}][\mathrm{TfO}]$

$V_{\text {cell }}\left(\mathrm{A}^{+} \mathrm{X}^{-}\right)=859.2 \AA^{3}$

$Z=6$ (trimeric asymmetric unit)

$\underline{\text { Volume }=0.133 \mathrm{~nm}^{3}}$

$[\mathrm{K}]^{+}$volume $=0.00986$, volume taken from Jenkins et al., Inorg Chem, 1999, 38, 3609.

6) $[\mathrm{Cs}][\mathrm{TfO}]$

$V_{\text {cell }}\left(\mathrm{A}^{+} \mathrm{X}^{-}\right)=317.8 \AA^{3}$

$Z=2$

$\underline{\text { Volume }=0.140 \mathrm{~nm}^{3}}$

$[\mathrm{Cs}]^{+}$volume $=0.01882$, volume taken from Jenkins et al., Inorg Chem, 1999, 38, 3609. 
$0.232 \pm 0.015 \mathrm{~nm}^{3}$ (3 salts considered)

1) $\left[\mathrm{NH}_{4}\right]\left[\mathrm{Tf}_{2} \mathrm{~N}\right]$

$V_{\text {cell }}\left(\mathrm{A}^{+} \mathrm{X}^{-}\right)=3990.0 \AA^{3}$

$Z=16$

$\underline{\text { Volume }=0.228 \mathrm{~nm}^{3}}$

$\left[\mathrm{NH}_{4}\right]^{+}$volume $=0.021 \pm 0.015 \mathrm{~nm}^{3}$ (3 salts considered), volume taken from Jenkins et al. Inorg Chem, 1999, 38, 3609.

2) $[\mathrm{K}]\left[\mathrm{Tf}_{2} \mathrm{~N}\right]$

$V_{\text {cell }}\left(\mathrm{A}^{+} \mathrm{X}^{-}\right)=3709.3 \AA^{3}$

$Z=16$

$\underline{\text { Volume }=0.222 \mathrm{~nm}^{3}}$

$[\mathrm{K}]^{+}$volume $=0.00986 \mathrm{~nm}^{3}$, volume taken from Jenkins et al., Inorg Chem, 1999, 38, 3609 .

3) $[\mathrm{Cs}]\left[\mathrm{Tf}_{2} \mathrm{~N}\right]$ (not included, as the

$V_{\text {cell }}\left(\mathrm{A}^{+} \mathrm{X}^{-}\right)=2125.1 \AA^{3}$

$Z=8$

$\underline{\text { Volume }=0.247 \mathrm{~nm}^{3}}$

$[\mathrm{Cs}]^{+}$volume $=0.01882 \mathrm{~nm}^{3}$, volume taken from Jenkins et al., Inorg Chem, 1999, 38, 3609. 


\section{Cations:}

\section{$[\mathrm{EMIM}]^{+}$}

\section{$0.156 \pm 0.018 \mathrm{~nm}^{3}$ (3 salts considered)}

1) $[\mathrm{EMIM}]\left[\mathrm{NO}_{3}\right]$

$V_{\text {cell }}\left(\mathrm{A}^{+} \mathrm{X}^{-}\right)=899.5 \AA^{3}$

$Z=4$

$\underline{\text { Volume }=0.161 \mathrm{~nm}^{3}}$

$\left[\mathrm{NO}_{3}\right]^{-}$volume $=0.064 \pm 0.011 \mathrm{~nm}^{3}$ (5 salts considered), volume taken from Jenkins et al , Inorg Chem, 1999, 38, 3609.

2) $[\mathrm{EMIM}]\left[\mathrm{NO}_{2}\right]$

$V_{\text {cell }}\left(\mathrm{A}^{+} \mathrm{X}^{-}\right)=412.8 \AA^{3}$

$Z=2$

$\underline{\text { Volume }=0.151 \mathrm{~nm}^{3}}$

$\left[\mathrm{NO}_{2}\right]^{-}$volume $=0.055 \pm 0.007 \mathrm{~nm}^{3}$ (3 salts considered), volume taken from Jenkins et al. Inorg Chem, 1999, 38, 3609.

3) $[\mathrm{EMIM}][\mathrm{Cl}]$

$V_{\text {cell }}\left(\mathrm{A}^{+} \mathrm{X}^{-}\right)=3240.0 \AA^{3}$

$Z=16$

$\underline{\text { Volume }=0.156 \mathrm{~nm}^{3}}$

$[\mathrm{Cl}]^{-}$volume $=0.047 \pm 0.013 \mathrm{~nm}^{3}$ (3 salts considered), volume taken from Jenkins et al., Inorg Chem, 1999, 38, 3609. 


\section{$\left[\mathrm{C}_{3} \mathrm{MIM}\right]^{+}$}

$178 \mathrm{~nm}^{3} \pm 0.028 \mathrm{~nm}^{3}$ (1 salt considered)

1) $\left[\mathrm{C}_{3} \mathrm{MIM}\right]_{2}\left[\mathrm{SiF}_{6}\right]$

$V_{\text {cell }}\left(\mathrm{A}^{+} \mathrm{X}^{-}\right)=934.1 \AA^{3}$

$Z=2$

$\underline{\text { Volume }=0.178 \mathrm{~nm}^{3}}$

$\left[\mathrm{SiF}_{6}\right]^{2-}$ volume $=0.112 \pm 0.028 \mathrm{~nm}^{3}$ (4 salts considered), volume taken from Jenkins et al. Inorg Chem, 1999, 38, 3609.

\section{$[\mathrm{BMIM}]^{+}$}

\section{$0.196 \pm 0.021 \mathrm{~nm}^{3}$ (3 salts considered)}

1) $[\mathrm{BMIM}]\left[\mathrm{PF}_{6}\right]$

$V_{\text {cell }}\left(\mathrm{A}^{+} \mathrm{X}^{-}\right)=610.2 \AA^{3}$

$Z=2$

$\underline{\text { Volume }=0.196 \mathrm{~nm}^{3}}$

$\left[\mathrm{PF}_{6}\right]^{-}$volume $=0.109 \pm 0.008 \mathrm{~nm}^{3}$ (4 salts considered), volume taken from Jenkins et al., Inorg Chem, 1999, 38, 3609.

2) $[\mathrm{BMIM}][\mathrm{Cl}]$

$V_{\text {cell }}\left(\mathrm{A}^{+} \mathrm{X}^{-}\right)=960.6 \AA^{3}$

$Z=4$

$\underline{\text { Volume }=0.193 \mathrm{~nm}^{3}}$

$[\mathrm{Cl}]^{-}$volume $=0.047 \pm 0.013 \mathrm{~nm}^{3}$ (3 salts considered), volume taken from Jenkins et al., Inorg Chem, 1999, 38, 3609. 
2) $[\mathrm{BMIM}][\mathrm{Br}]$

$V_{\text {cell }}\left(\mathrm{A}^{+} \mathrm{X}^{-}\right)=1025.8 \AA^{3}$

$Z=4$

$\underline{\text { Volume }=0.200 \mathrm{~nm}^{3}}$

$[\mathrm{Br}]^{-}$volume $=0.056 \pm 0.014 \mathrm{~nm}^{3}$ (4 salts considered), volume taken from Jenkins et al., Inorg Chem, 1999, 38, 3609.

\section{$\left[\mathrm{C}_{5} \mathrm{MIM}\right]^{+}$}

\section{$0.219 \pm 0.015 \mathrm{~nm}^{3}$}

Estimated from the average volume change of $0.023 \mathrm{~nm}^{3}$ on adding a " $\mathrm{CH}_{2}$ " group in the series $[\mathrm{MMIM}]^{+},[\mathrm{EMIM}]^{+},\left[\mathrm{C}_{3} \mathrm{MIM}\right]^{+},[\mathrm{BMIM}]^{+} .($Added $4 * 0.023$ to MMIM volume $)$

Volume of $[\mathrm{MMIM}]^{+}=0.127 \pm 0.015 \mathrm{~nm}^{3}$

1) $[\mathrm{MMIM}][\mathrm{Cl}]$

$V_{\text {cell }}\left(\mathrm{A}^{+} \mathrm{X}^{-}\right)=687.8 \AA^{3}$

$Z=4$

$\left[\mathrm{MMIM}^{+}\right.$Volume $=0.125 \mathrm{~nm}^{3}$

$[\mathrm{Cl}]^{-}$volume $=0.047 \pm 0.013 \mathrm{~nm}^{3}$ (3 salts considered), volume taken from Jenkins et al., Inorg

Chem, 1999, 38, 3609.

2) $[\mathrm{MMIM}]\left[\mathrm{PF}_{6}\right]$

$V_{\text {cell }}\left(\mathrm{A}^{+} \mathrm{X}^{-}\right)=1893.9 \AA^{3}$

$Z=8$

$\left[\mathrm{MMIM}^{+}\right.$Volume $=0.128 \mathrm{~nm}^{3}$

$\left[\mathrm{PF}_{6}\right]^{-}$volume $=0.109 \pm 0.008 \mathrm{~nm}^{3}$ (4 salts considered), volume taken from Jenkins et al. Inorg Chem, 1999, 38, 3609. 


\section{[BMMIM $^{+}$}

\section{$0.229 \pm 0.012 \mathrm{~nm}^{3}$ (3 salts considered)}

1) $[\mathrm{BMMIM}]\left[\mathrm{BF}_{4}\right]$

$V_{\text {cell }}\left(\mathrm{A}^{+} \mathrm{X}^{-}\right)=311.5 \AA^{3}$

$Z=1$

$\underline{\text { Volume }=0.239 \mathrm{~nm}^{3}}$

$\left[\mathrm{BF}_{4}\right]^{-}$volume $=0.073 \pm 0.009 \mathrm{~nm}^{3}$ (5 salts considered), volume taken from Jenkins et al. Inorg Chem, 1999, 38, 3609.

2) $[\mathrm{BMMIM}]\left[\mathrm{PF}_{6}\right]$

$V_{\text {cell }}\left(\mathrm{A}^{+} \mathrm{X}^{-}\right)=327.6 \AA^{3}$

$Z=1$

$\underline{\text { Volume }=0.219 \mathrm{~nm}^{3}}$

$\left[\mathrm{PF}_{6}\right]^{-}$volume $=0.109 \pm 0.008 \mathrm{~nm}^{3}$ (4 salts considered), volume taken from Jenkins et al. Inorg Chem, 1999, 38, 3609.

3) $[\mathrm{BMMIM}]\left[\mathrm{SbF}_{6}\right]$ (not included, as the uncertainty on the volume of the $\left[\mathrm{SbF}_{6}\right]^{-}$anion is very large.

$V_{\text {cell }}\left(\mathrm{A}^{+} \mathrm{X}^{-}\right)=364.3 \AA^{3}$

$Z=1$

$\underline{\text { Volume }=0.243 \mathrm{~nm}^{3}}$

$\left[\mathrm{SbF}_{6}\right]^{-}$volume $=0.121 \pm 0.112 \mathrm{~nm}^{3}$ (6 salts considered), volume taken from Jenkins et al. Inorg Chem, 1999, 38, 3609. 
$[\mathrm{BPy}]^{+}$

$0.198 \mathrm{~nm}^{3} \pm 0.013$ (1 salt considered)

1) $[\mathrm{BPy}][\mathrm{Cl}]$

$V_{\text {cell }}\left(\mathrm{A}^{+} \mathrm{X}^{-}\right)=245.4 \AA^{3}$

$Z=1$

$\underline{\text { Volume }=0.198 \mathrm{~nm}^{3}}$

$[\mathrm{Cl}]^{-}$volume $=0.047 \pm 0.013 \mathrm{~nm}^{3}$ (3 salts considered), volume taken from Jenkins et al., Inorg Chem, 1999, 38, 3609 .

\section{$[\mathrm{BMPyr}]^{+}$}

\section{$0.221 \pm 0.015 \mathrm{~nm}^{3}$ (1 salt considered)}

1) $[\mathrm{BMPyr}]\left[\mathrm{Tf}_{2} \mathrm{~N}\right]$

$V_{\text {cell }}\left(\mathrm{A}^{+} \mathrm{X}^{-}\right)=1811.1 \AA^{3}$

$Z=4$

Volume $=0.221 \mathrm{~nm}^{3}$

$\left[\mathrm{Tf}_{2} \mathrm{~N}\right]^{-}$volume $=0.232 \pm 0.015 \mathrm{~nm}^{3}$ (3 salts considered), volume derived above.

\section{$\left[\mathrm{C}_{5} \mathrm{MPyr}\right]^{+}$}

\section{$0.238 \pm 0.018 \mathrm{~nm}^{3}$}

Estimated from $[\mathrm{MMPyr}]^{+}$volume (as this is calculated from the largest number of salts available for methyl pyrolidinium cations) based on an average volume of $0.023 \mathrm{~nm}^{3}$ for the addition or subtraction of " $\mathrm{CH}_{2}$ " groups in the pyrolidinium and ammonium salts below:

[MMPyr] Volume $=146 \pm 0.018 \mathrm{~nm}^{3}(2$ salts considered $)$

1) $[\mathrm{MMPyr}]\left[\mathrm{PF}_{6}\right]$ 
$V_{\text {cell }}\left(\mathrm{A}^{+} \mathrm{X}^{-}\right)=507.2 \AA^{3}$

$Z=2$

Volume $=0.145 \mathrm{~nm}^{3}$

$\left[\mathrm{PF}_{6}\right]^{-}$volume $=0.109 \pm 0.008 \mathrm{~nm}^{3}$ (4 salts considered), volume taken from Jenkins et al., Inorg Chem, 1999, 38, 3609.

2) [MMPyr][I]

$V_{\text {cell }}\left(\mathrm{A}^{+} \mathrm{X}^{-}\right)=874.0 \AA^{3}$

$Z=4$

$\underline{\text { Volume }=0.147 \mathrm{~nm}^{3}}$

$\left[\mathrm{I}^{-}\right.$volume $=0.072 \pm 0.016 \mathrm{~nm}^{3}$ (4 salts considered), volume taken from Jenkins et al., Inorg Chem, 1999, 38, 3609.

3) $\left[\mathrm{C}_{3} \mathrm{MPyr}\right]\left[\mathrm{PF}_{6}\right]$

$V_{\text {cell }}\left(\mathrm{A}^{+} \mathrm{X}^{-}\right)=10922.0 \AA^{3}$

$Z=36$

$\underline{\text { Volume }=0.194 \mathrm{~nm}^{3}}$

$\left[\mathrm{PF}_{6}\right]^{-}$volume $=0.109 \pm 0.008 \mathrm{~nm}^{3}$ (4 salts considered), volume taken from Jenkins et al., Inorg Chem, 1999, 38, 3609.

4) $\left[\mathrm{C}_{7} \mathrm{MPyr}\right]\left[\mathrm{PF}_{6}\right]$

$V_{\text {cell }}\left(\mathrm{A}^{+} \mathrm{X}^{-}\right)=1623.6 \AA^{3}$

$Z=4$

$\underline{\text { Volume }=0.297 \mathrm{~nm}^{3}}$

$\left[\mathrm{PF}_{6}\right]^{-}$volume $=0.109 \pm 0.008 \mathrm{~nm}^{3}$ (4 salts considered), volume taken from Jenkins et al. Inorg Chem, 1999, 38, 3609. 
5) $\left[\mathrm{NMe}_{4}\right]^{+}$

volume $=0.113 \pm 0.013 \mathrm{~nm}^{3}$ (3 salts considered), volume taken from Jenkins et al., Inorg Chem, 1999, 38, 3609.

6) $\left[\mathrm{NEt}_{4}\right]^{+}$ volume $=0.199 \pm 0.016 \mathrm{~nm}^{3}$ (1 salt considered $)$, volume taken from Jenkins et al., Inorg Chem, 1999, 38, 3609.

7) $\left[\mathrm{NBu}_{4}\right]$ volume $=0.383 \pm 0.008 \mathrm{~nm}^{3}(2$ salt considered $)$

a) $\left[\mathrm{NBu}_{4}\right]\left[\mathrm{PF}_{6}\right]$

$V_{\text {cell }}\left(\mathrm{A}^{+} \mathrm{X}^{-}\right)=2021.1 \AA^{3}$

$Z=4$

$\underline{\text { Volume }=0.396 \mathrm{~nm}^{3}}$

$\left[\mathrm{PF}_{6}\right]^{-}$volume $=0.109 \pm 0.008 \mathrm{~nm}^{3}$ (4 salts considered), volume taken from Jenkins et al. Inorg Chem, 1999, 38, 3609.

b) $\left[\mathrm{NBu}_{4}\right]\left[\mathrm{PF}_{6}\right]$

$V_{\text {cell }}\left(\mathrm{A}^{+} \mathrm{X}^{-}\right)=1914.9 \AA^{3}$

$Z=4$

$\underline{\text { Volume }=0.370 \mathrm{~nm}^{3}}$

$\left[\mathrm{PF}_{6}\right]^{-}$volume $=0.109 \pm 0.008 \mathrm{~nm}^{3}$ (4 salts considered), volume taken from Jenkins et al. Inorg Chem, 1999, 38, 3609. 


\section{$\left[\mathrm{C}_{5} \mathrm{NEt}_{3}\right]^{+}$}

\section{$0.268 \pm 0.016 \mathrm{~nm}^{3}$}

Estimated from $\left[\mathrm{NEt}_{4}\right]^{+}$volume $\left(0.199 \pm 0.016 \mathrm{~nm}^{3}\right.$ (1 salt considered), volume taken from Jenkins et al., Inorg Chem, 1999, 38, 3609.) based on an average volume of $0.023 \mathrm{~nm}^{3}$ for the addition or subtraction of " $\mathrm{CH}_{2}$ " groups in the pyrolidinium and ammonium salts above:

\section{Part 2 - Using Hofmann's Average Atomic Volumes}

A selection of elemental volumes for elements commonly found in ionic liquids is shown below errors are indicated in brackets. ${ }^{\mathrm{ix}}$

\begin{tabular}{cccc} 
Element & Volume $\left(\AA^{3}\right)$ & Element & Volume $\left(\AA^{3}\right)$ \\
\hline $\mathrm{C}$ & $13.87(0.05)$ & $\mathrm{O}$ & $11.39(0.17)$ \\
$\mathrm{H}$ & $5.08(0.04)$ & $\mathrm{F}$ & $11.17(0.15)$ \\
$\mathrm{N}$ & $11.8(0.3)$ & $\mathrm{P}$ & $29.5(0.2)$ \\
$\mathrm{B}$ & $13.24(0.17)$ & $\mathrm{S}$ & $25.2(0.2)$
\end{tabular}

Estimated volumes, using Hofmann's atomic volumes (ATM), for the ions in this paper are shown below and compared to volumes derived from X-ray structures (XRD). Standard deviations are indicated in brackets.

\begin{tabular}{|cccc|cccc|}
\hline $\begin{array}{c}\text { Cations } \\
(\mathrm{XRD})\end{array}$ & $\begin{array}{c}V_{\text {ion }} \\
\left(\mathrm{XRD} / \mathrm{nm}^{3}\right)\end{array}$ & $\begin{array}{c}\text { Anions } \\
(\mathrm{XRD})\end{array}$ & $\begin{array}{c}V_{\text {ion }} \\
\left(\mathrm{XRD} / \mathrm{nm}^{3}\right)\end{array}$ & $\begin{array}{c}\text { Cations } \\
(\mathrm{ATM})\end{array}$ & $\begin{array}{c}V_{\text {ion }} \\
\left(\mathrm{ATM} / \mathrm{nm}^{3}\right)\end{array}$ & $\begin{array}{c}\text { Anions } \\
(\mathrm{ATM})\end{array}$ & $\begin{array}{c}V_{\text {ion }} \\
\left(\mathrm{ATM} / \mathrm{nm}^{3}\right)\end{array}$ \\
\hline$[\mathrm{EMIM}]^{+}$ & $0.156(0.018)$ & {$\left[\mathrm{BF}_{4}\right]^{-}$} & $0.073(0.009)$ & {$[\mathrm{EMIM}]^{+}$} & $0.163(0.0005)$ & {$\left[\mathrm{BF}_{4}\right]^{-}$} & $0.058(0.0004)$ \\
{$\left[\mathrm{C}_{3} \mathrm{MIM}\right]^{+}$} & $0.178(0.028)$ & {$\left[\mathrm{PF}_{6}\right]^{-}$} & $0.109(0.008)$ & {$\left[\mathrm{C}_{3} \mathrm{MIM}\right]^{+}$} & $0.187(0.0005)$ & {$\left[\mathrm{PF}_{6}\right]^{-}$} & $0.097(0.0003)$ \\
{$[\mathrm{BMIM}]^{+}$} & $0.196(0.021)$ & {$\left[\mathrm{TfO}^{-}\right.$} & $0.131(0.015)$ & {$[\mathrm{BMIM}]^{+}$} & $0.216(0.0005)$ & {$\left[\mathrm{TfO}^{-}\right.$} & $0.107(0.0004)$ \\
{$\left[\mathrm{C}_{5} \mathrm{MIM}\right]^{+}$} & $0.219(0.015)$ & {$\left[\mathrm{Tf}_{2} \mathrm{~N}\right]^{-}$} & $0.232(0.015)$ & {$\left[\mathrm{C}_{5} \mathrm{MIM}\right]^{+}$} & $0.245(0.0005)$ & {$\left[\mathrm{Tf}_{2} \mathrm{~N}\right]^{-}$} & $0.203(0.0007)$ \\
{$[\mathrm{BMMIM}]^{+}$} & $0.229(0.012)$ & & & {$[\mathrm{BMMIM}]^{+}$} & $0.240(0.0005)$ & & \\
{$[\mathrm{BPy}]^{+}$} & $0.198(0.013)$ & & & {$[\mathrm{BPy}]^{+}$} & $0.184(0.0004)$ & & \\
{$[\mathrm{BMPyr}]^{+}$} & $0.221(0.015)$ & & & {$[\mathrm{BMPyr}]^{+}$} & $0.214(0.0004)$ & & \\
{$\left[\mathrm{C}_{5} \mathrm{MPyr}^{+}\right.$} & $0.238(0.018)$ & & & $\mathrm{C}_{5} \mathrm{MPyr}^{+}$ & $0.262(0.0004)$ & & \\
{$\left[\mathrm{C}_{5} \mathrm{NEt}_{3}\right]^{+}$} & $0.268(0.016)$ & & & {$\left[\mathrm{C}_{5} \mathrm{NEt}\right]^{+}$} & $0.243(0.0004)$ & & \\
\hline
\end{tabular}




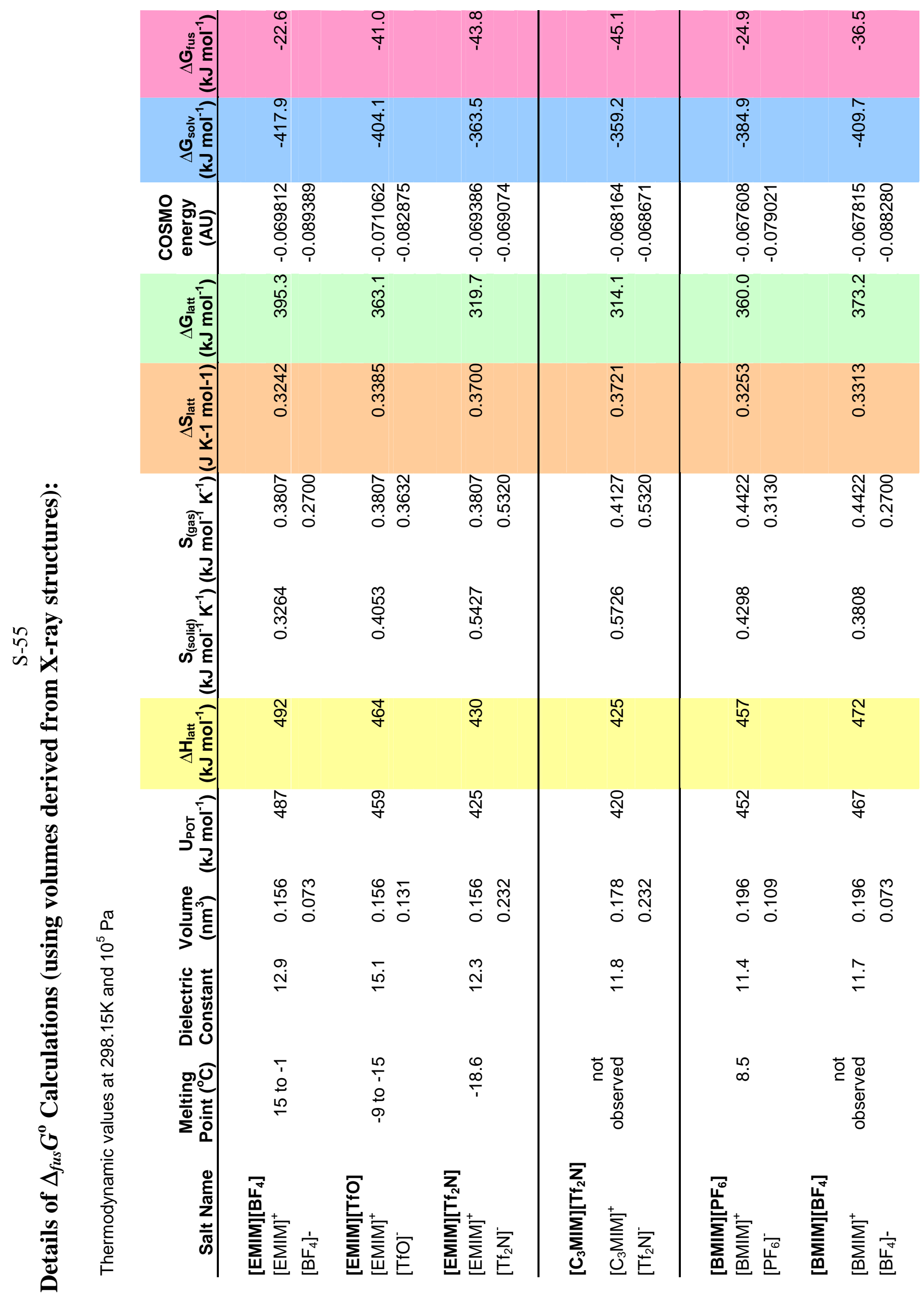




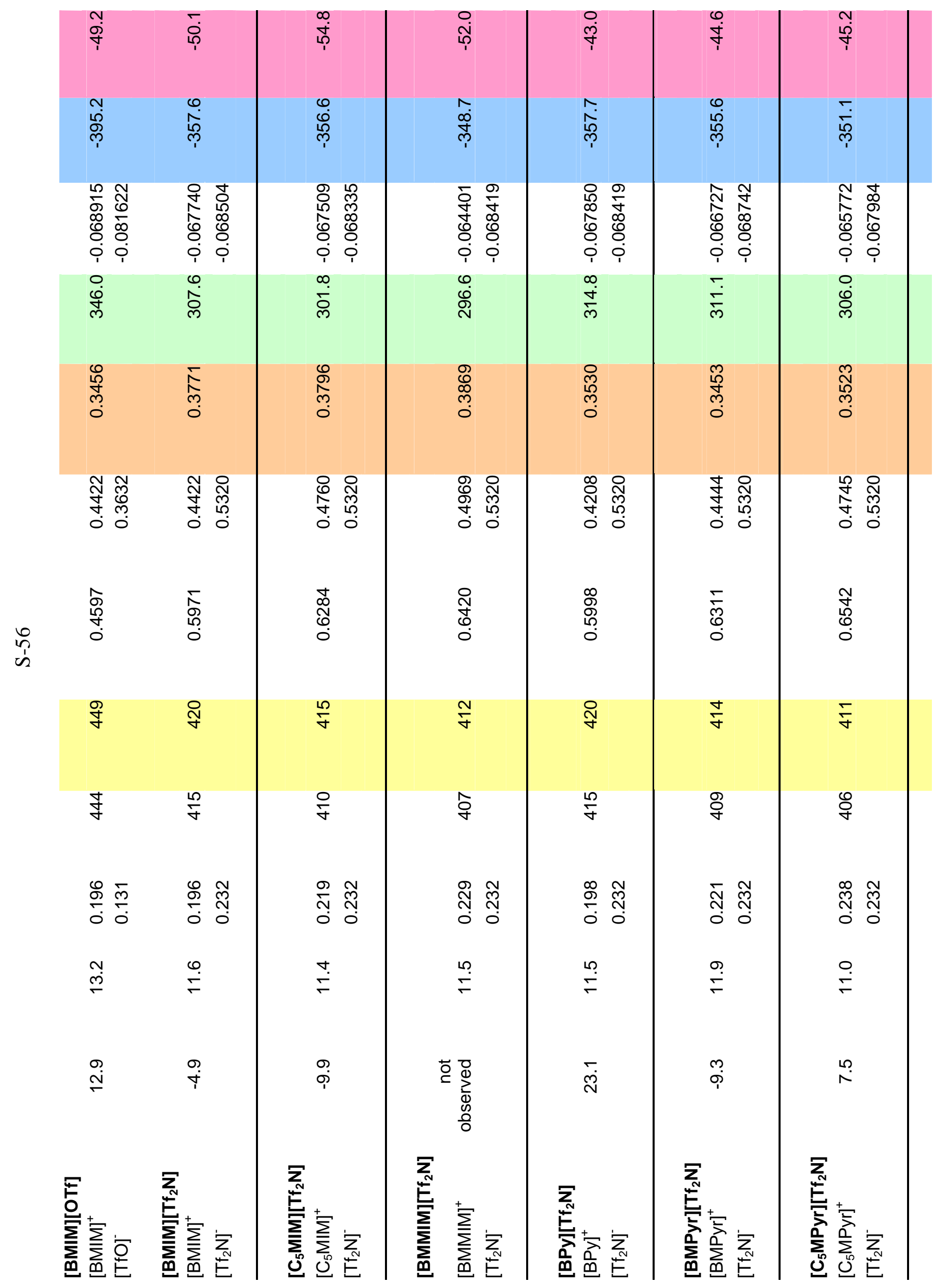




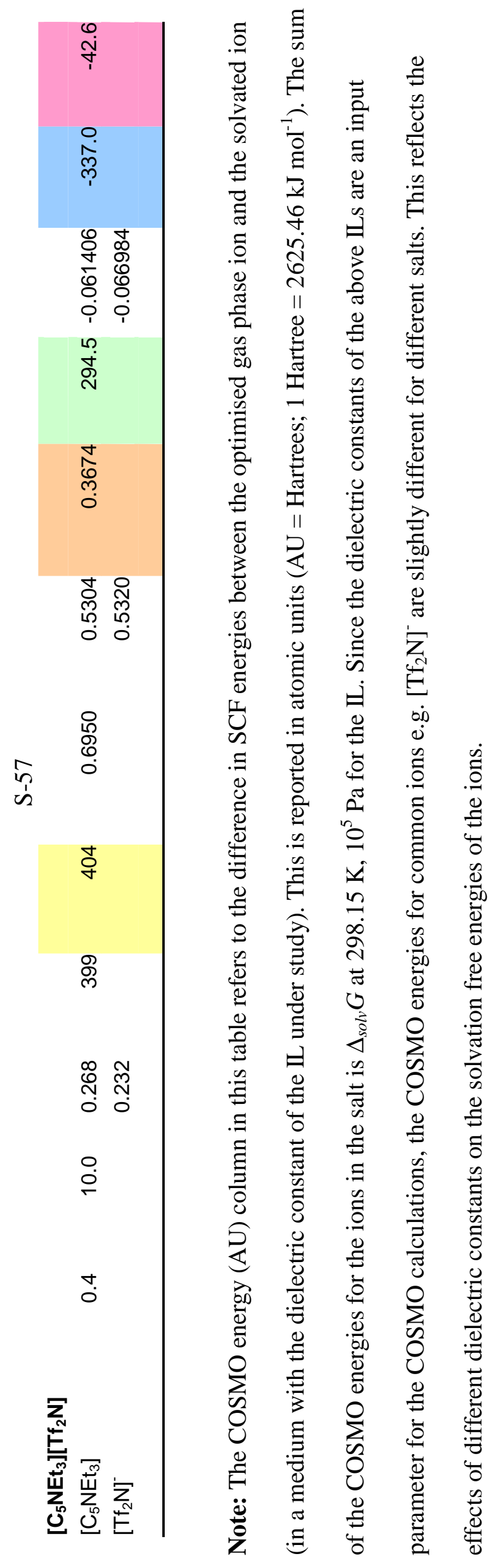




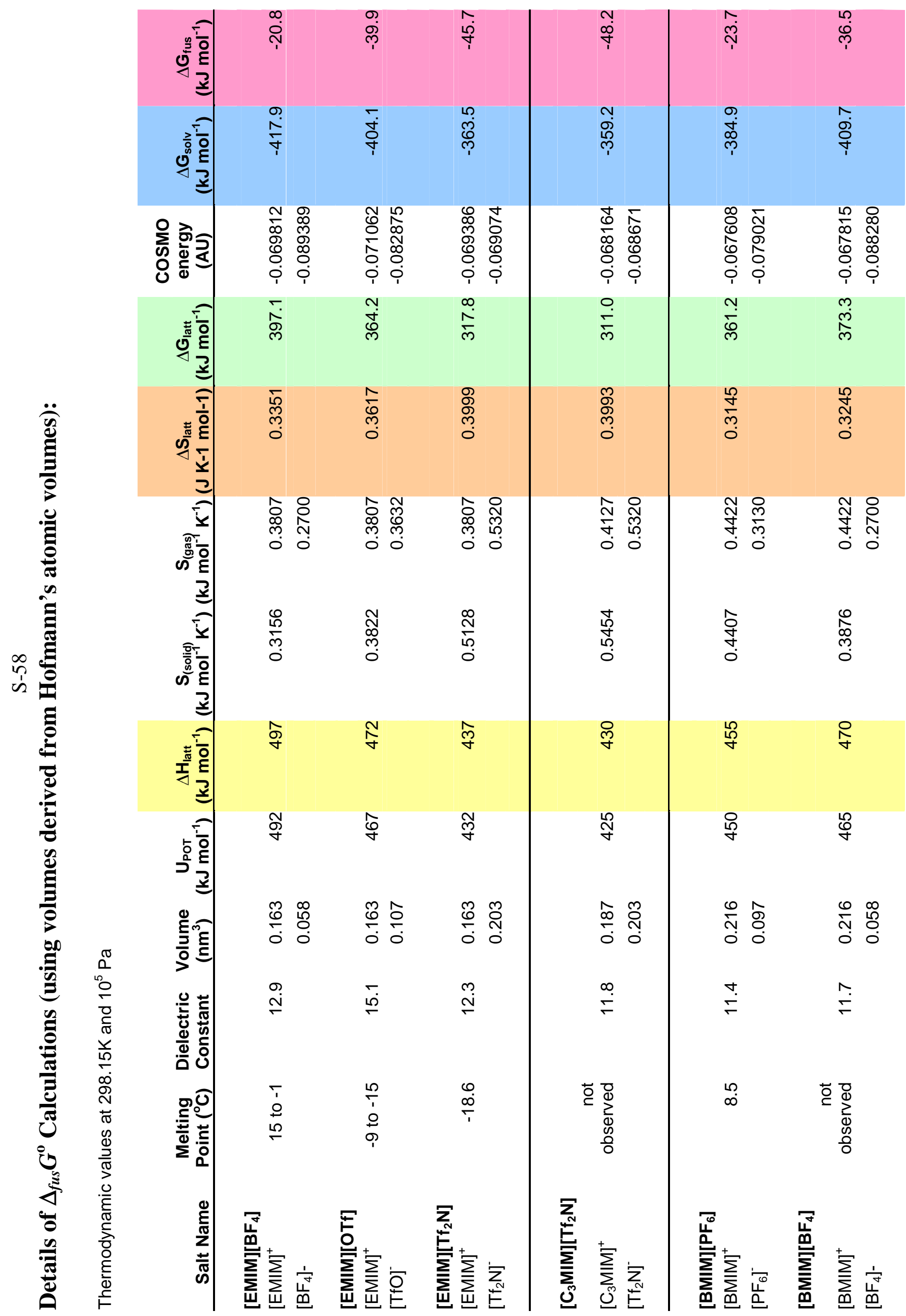




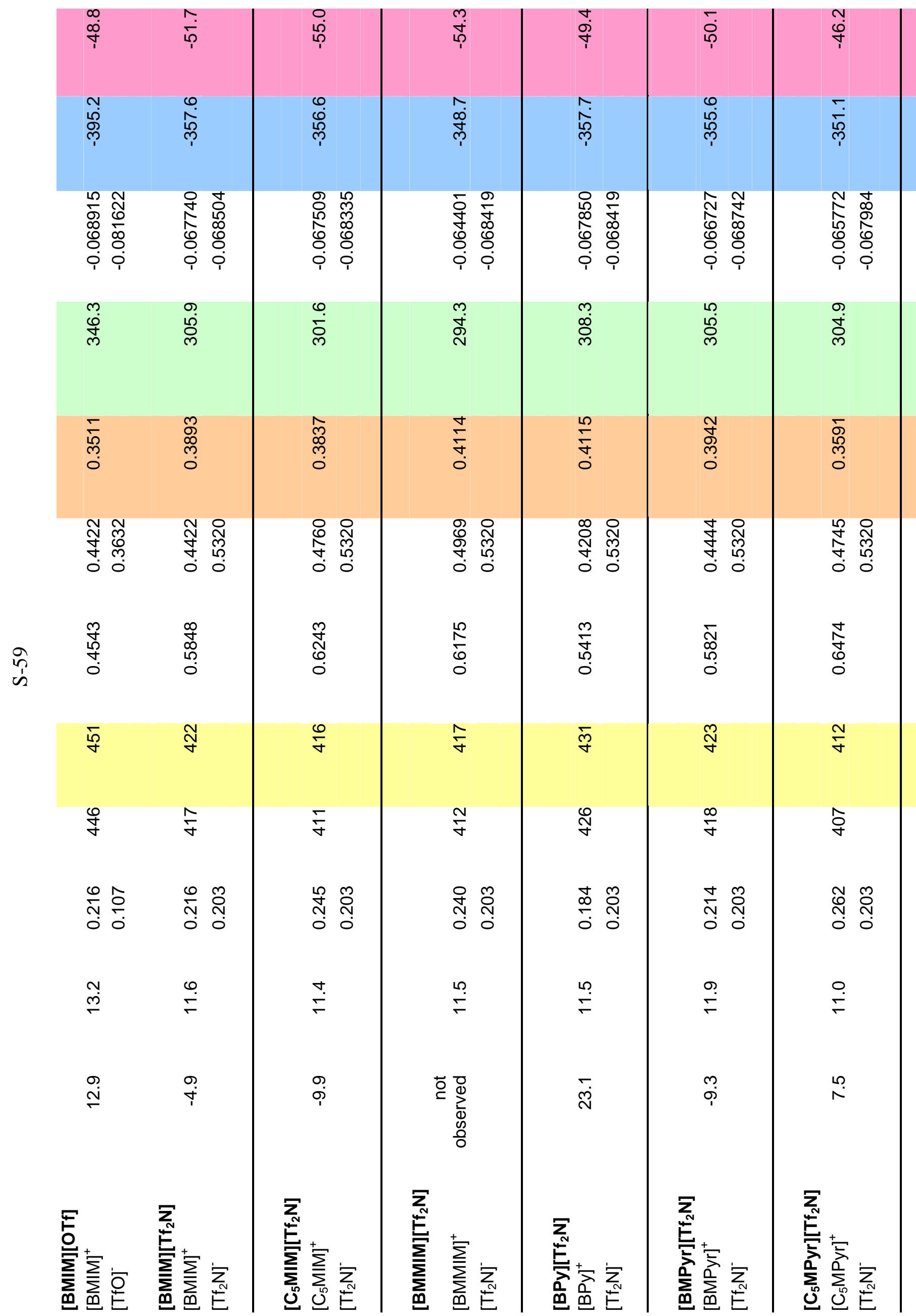




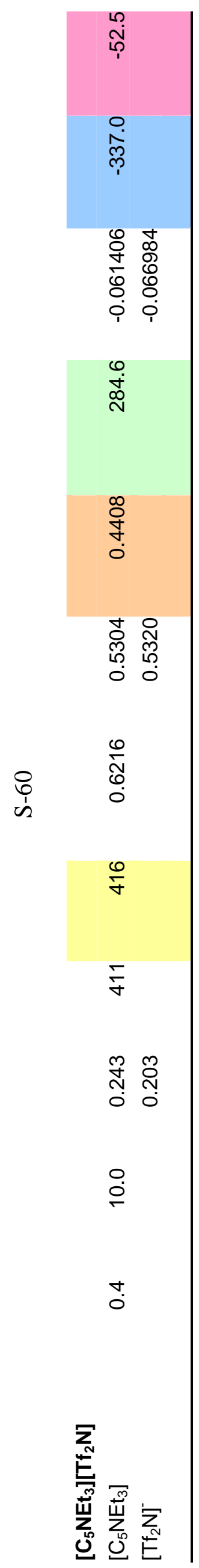




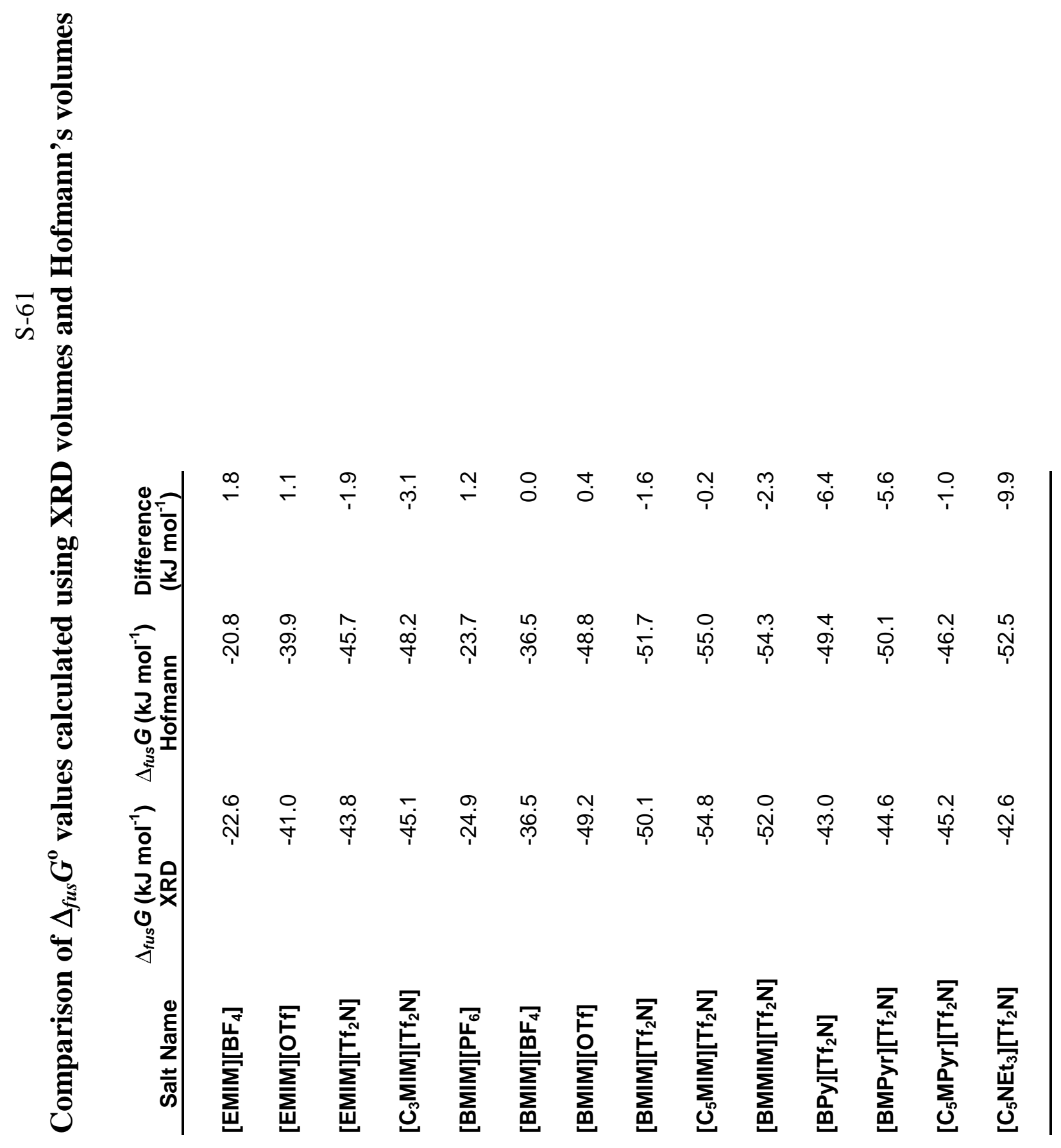




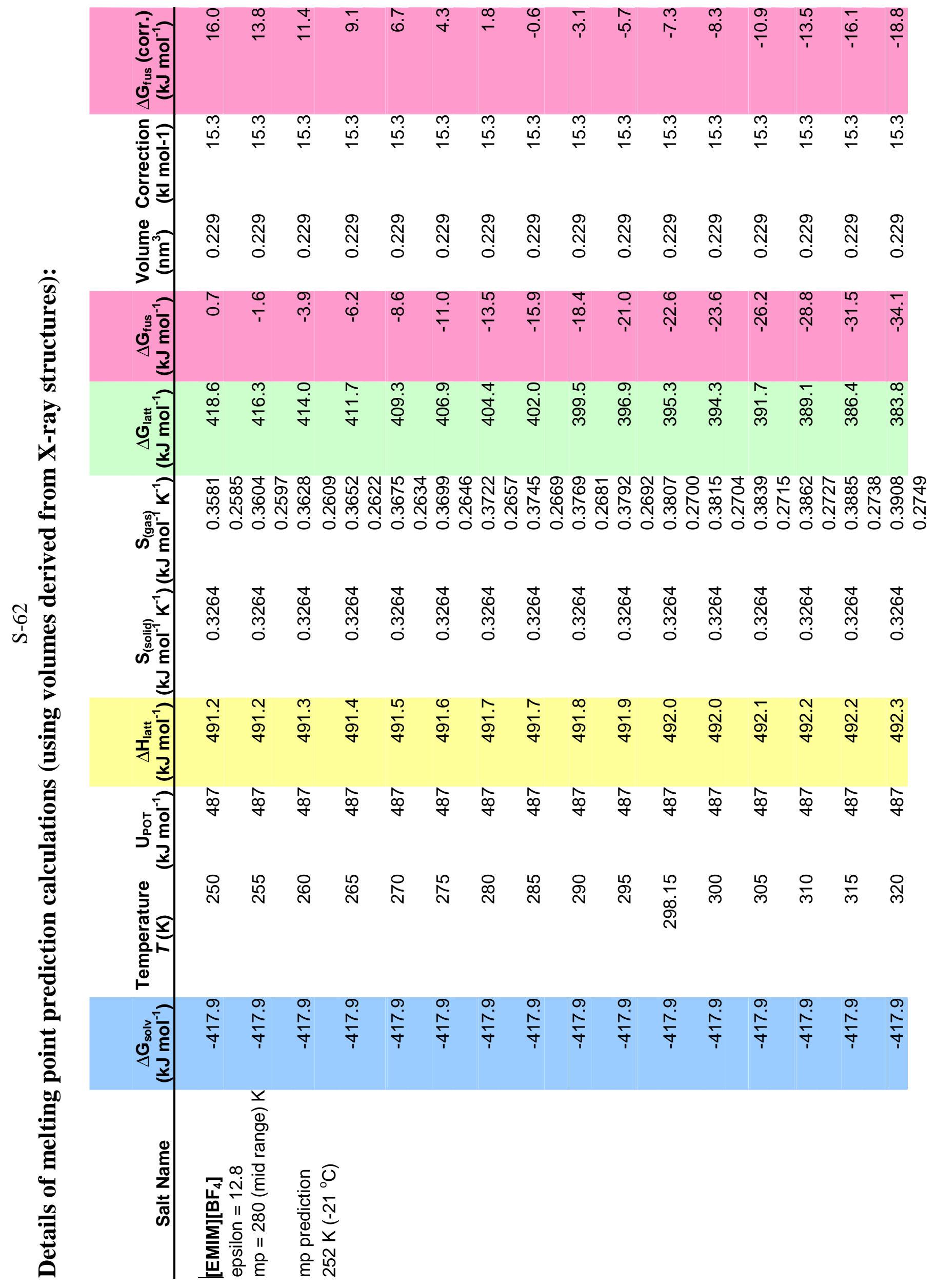




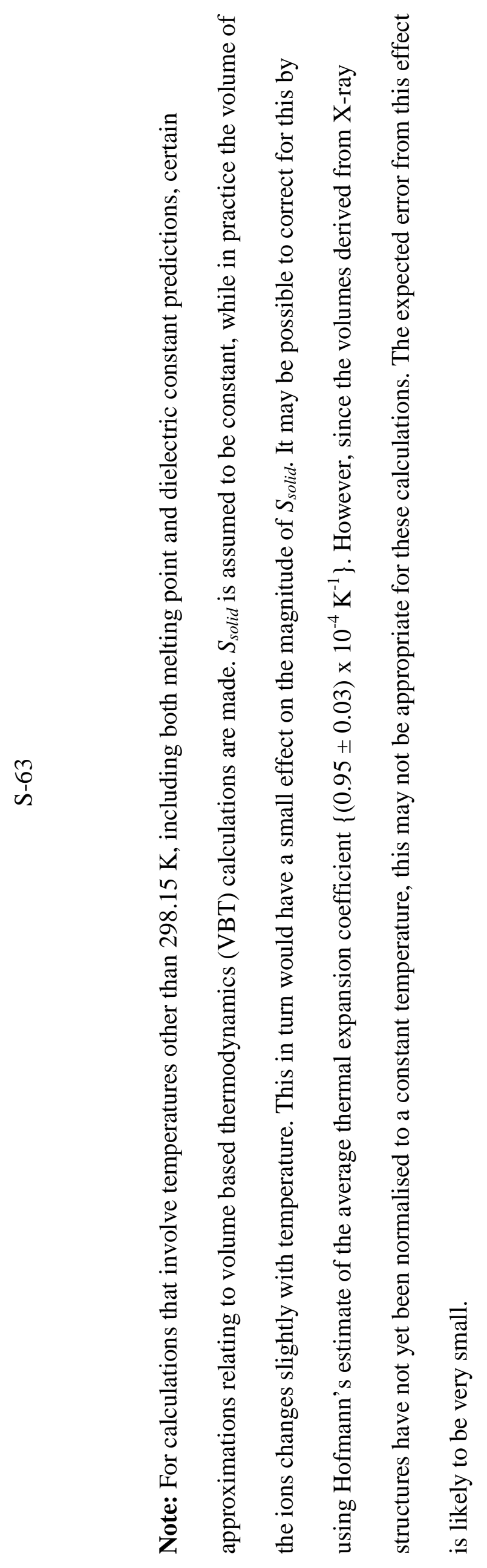



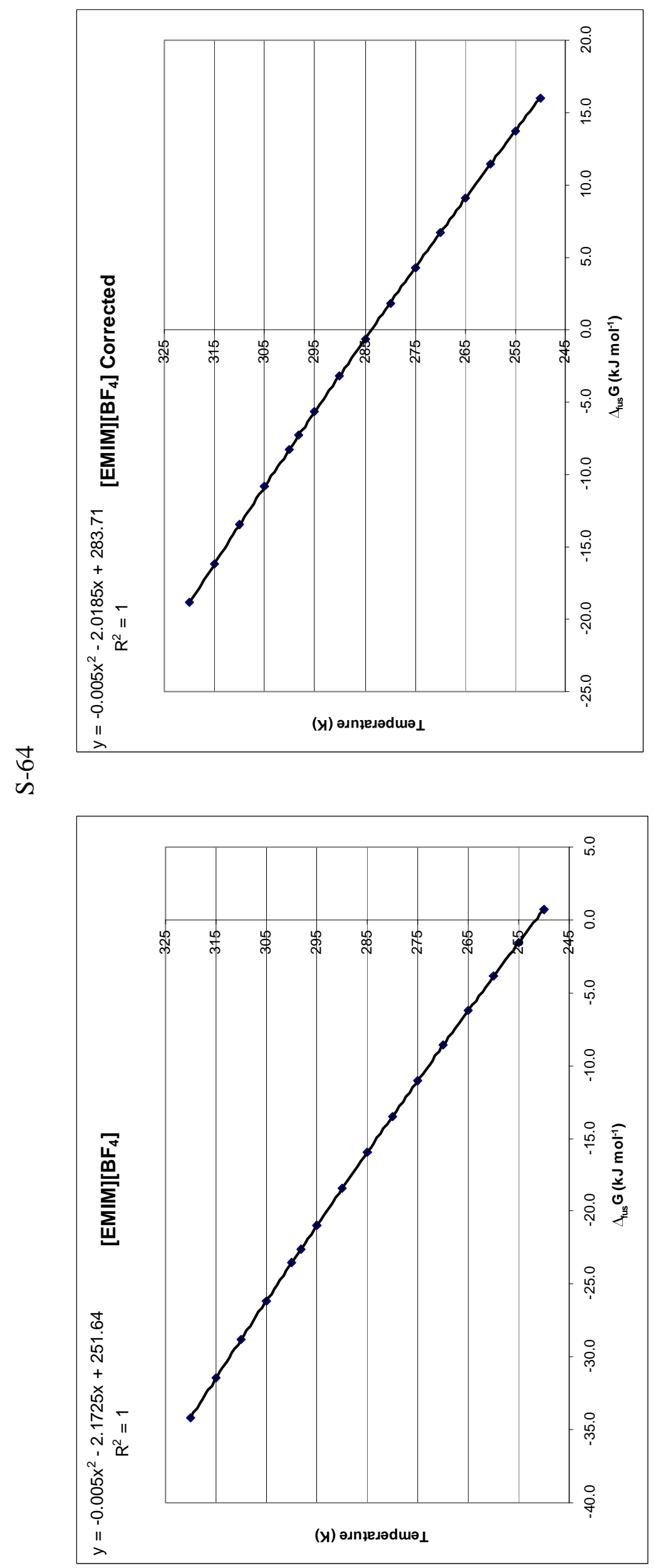


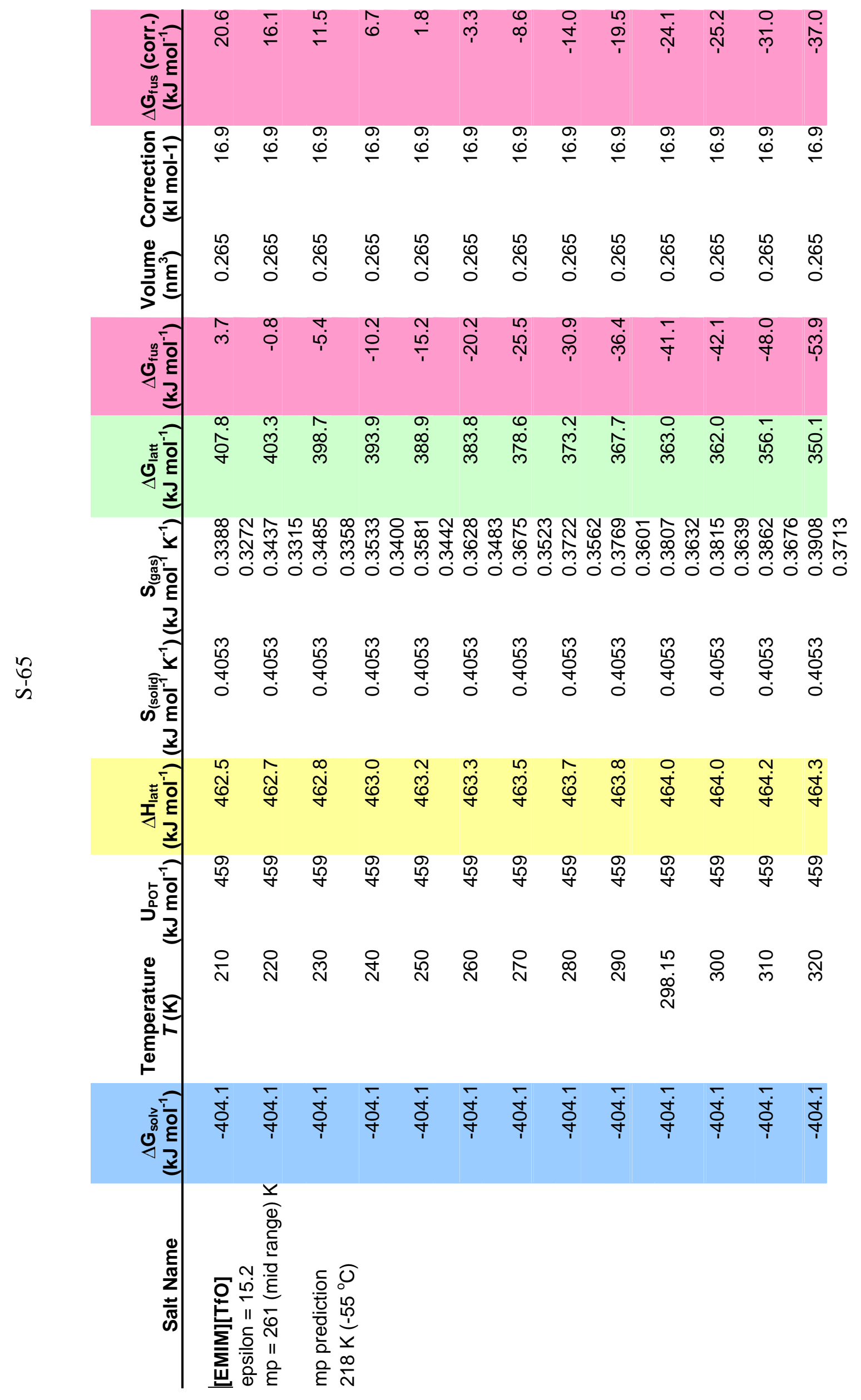




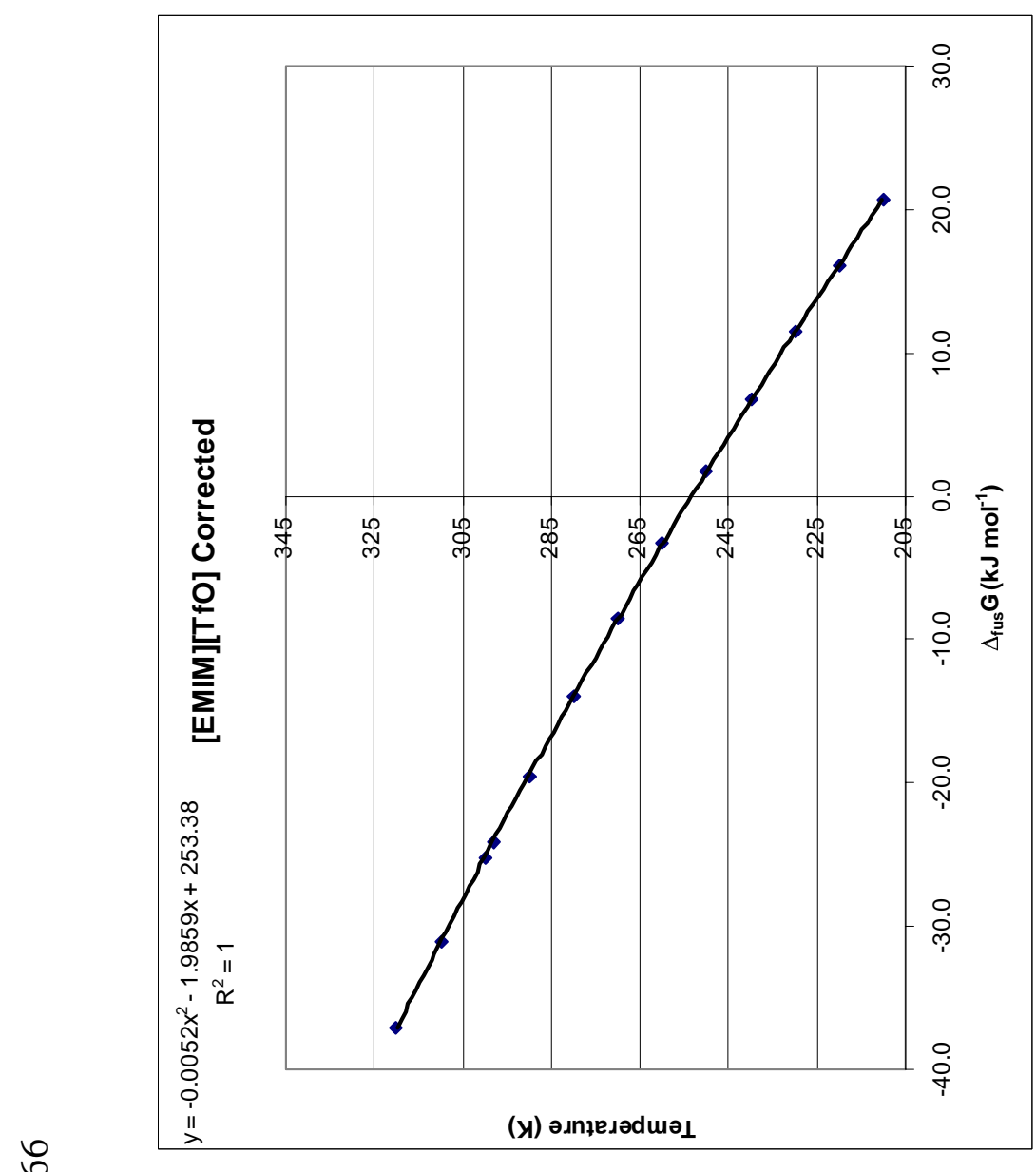

0
is

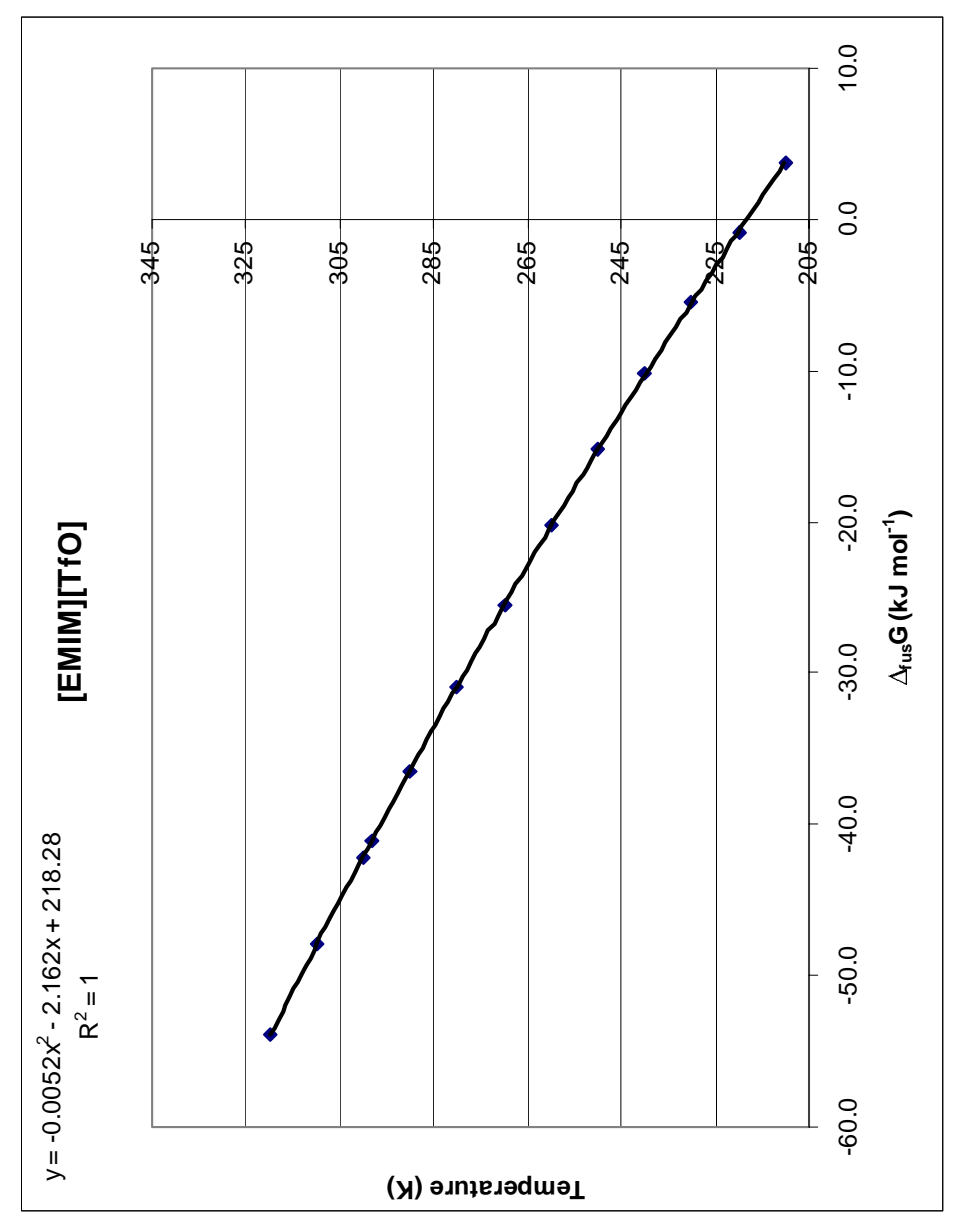




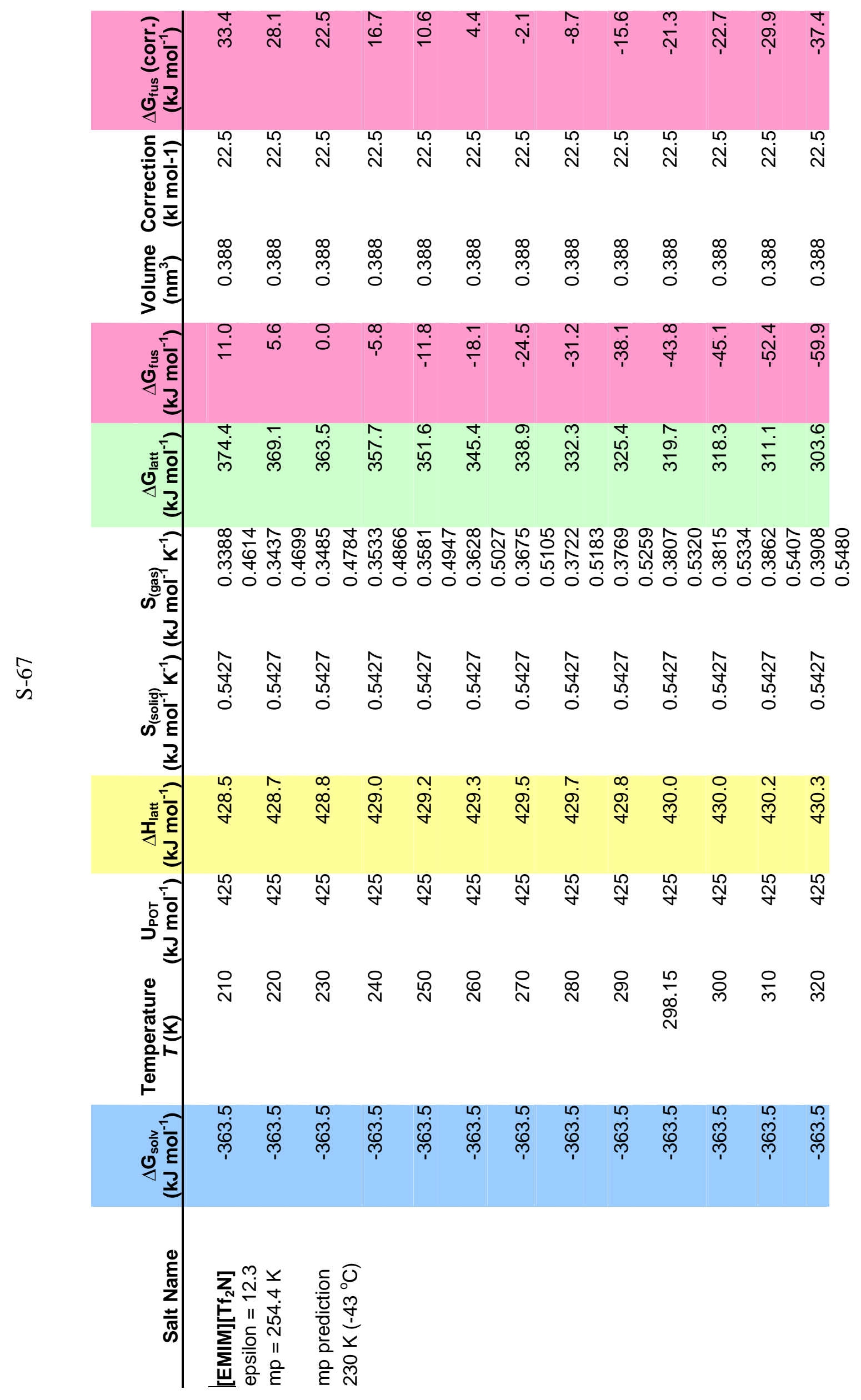




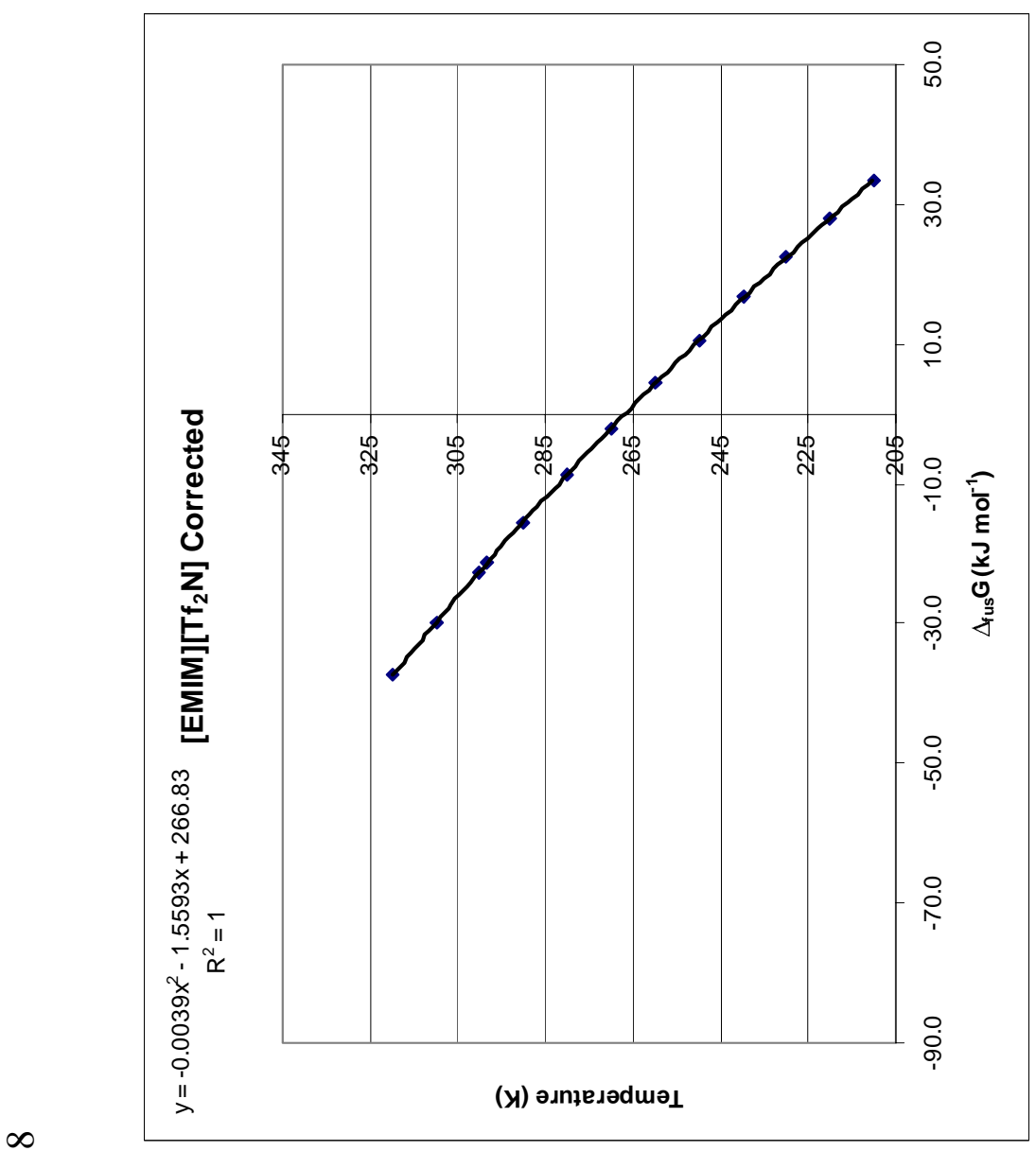

$\infty$
0
1
1

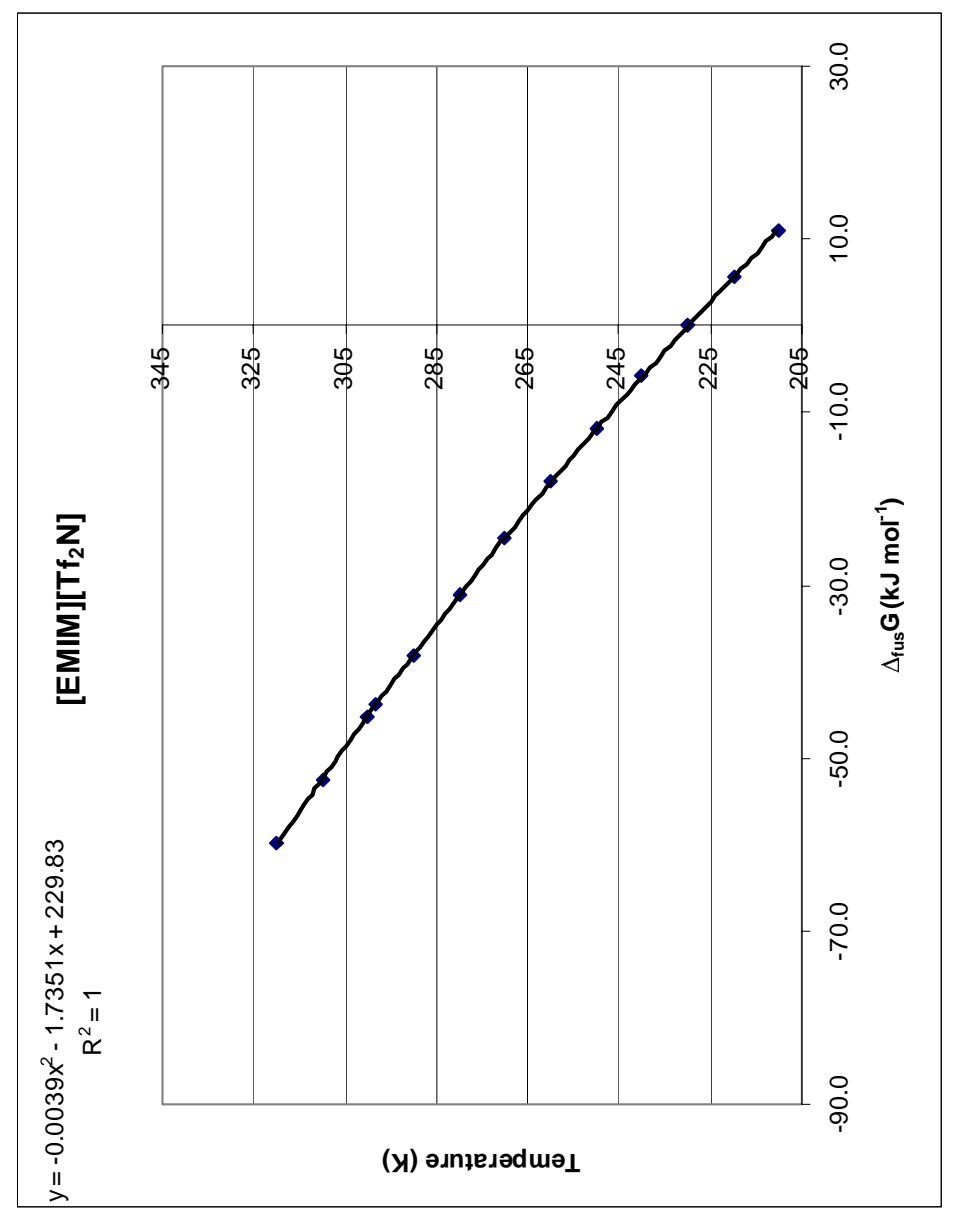




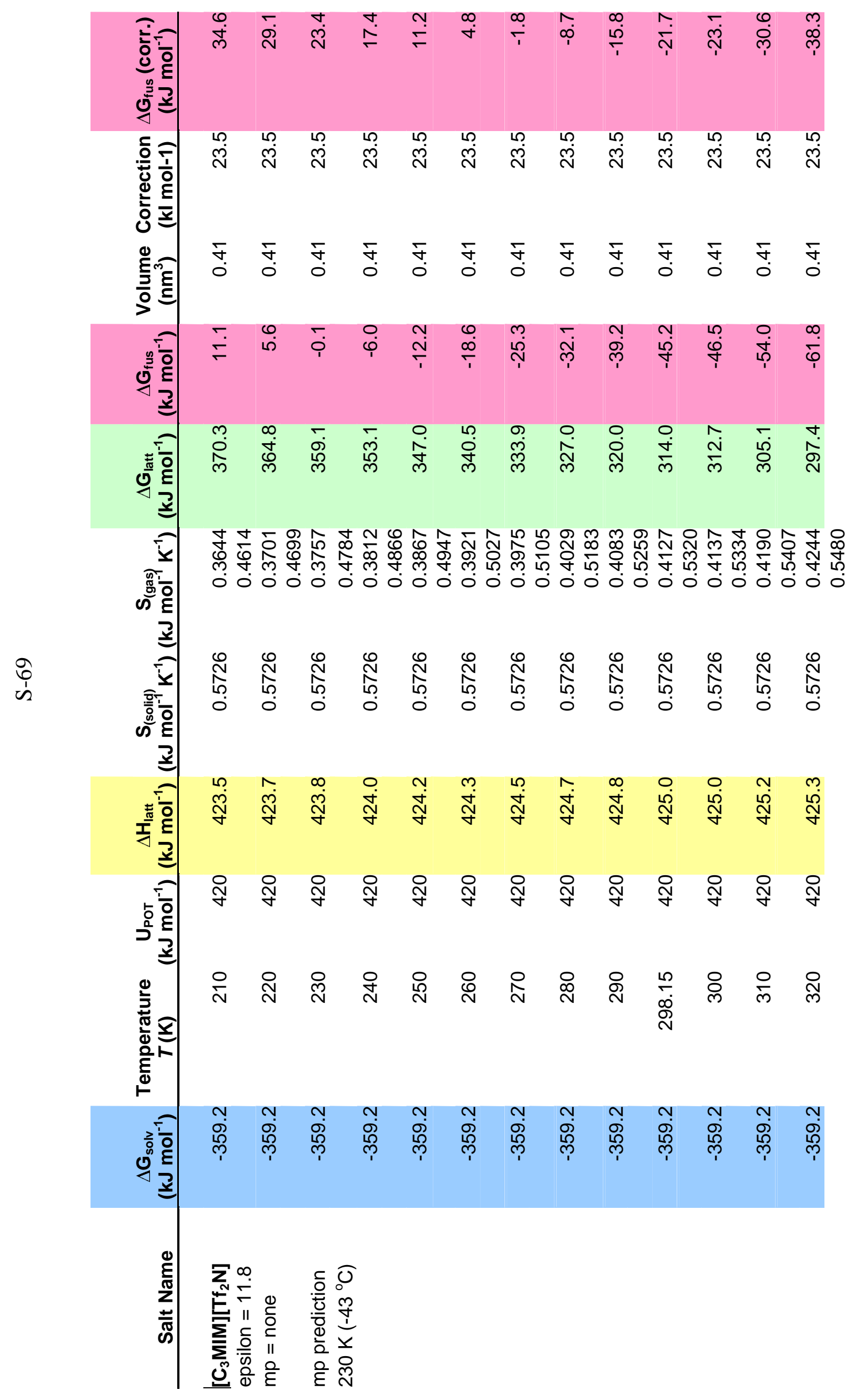




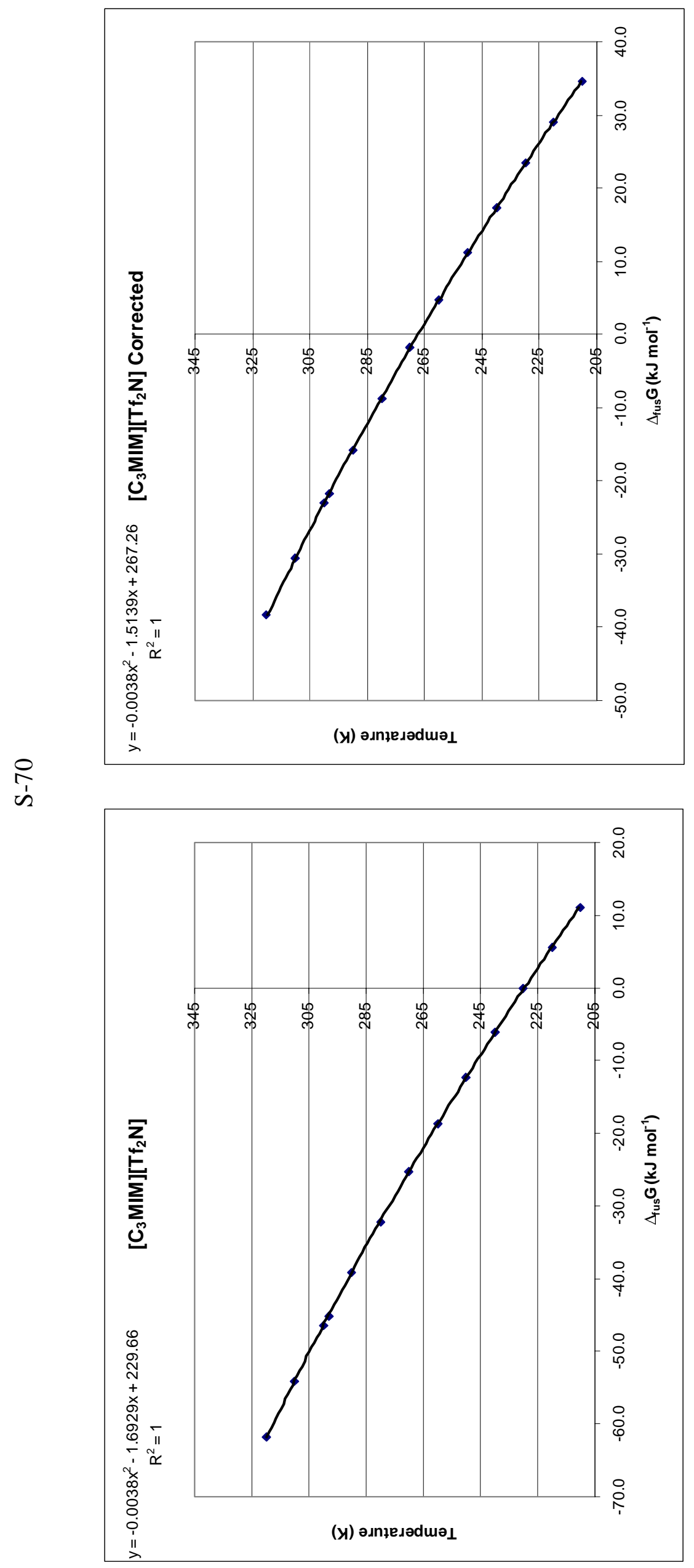




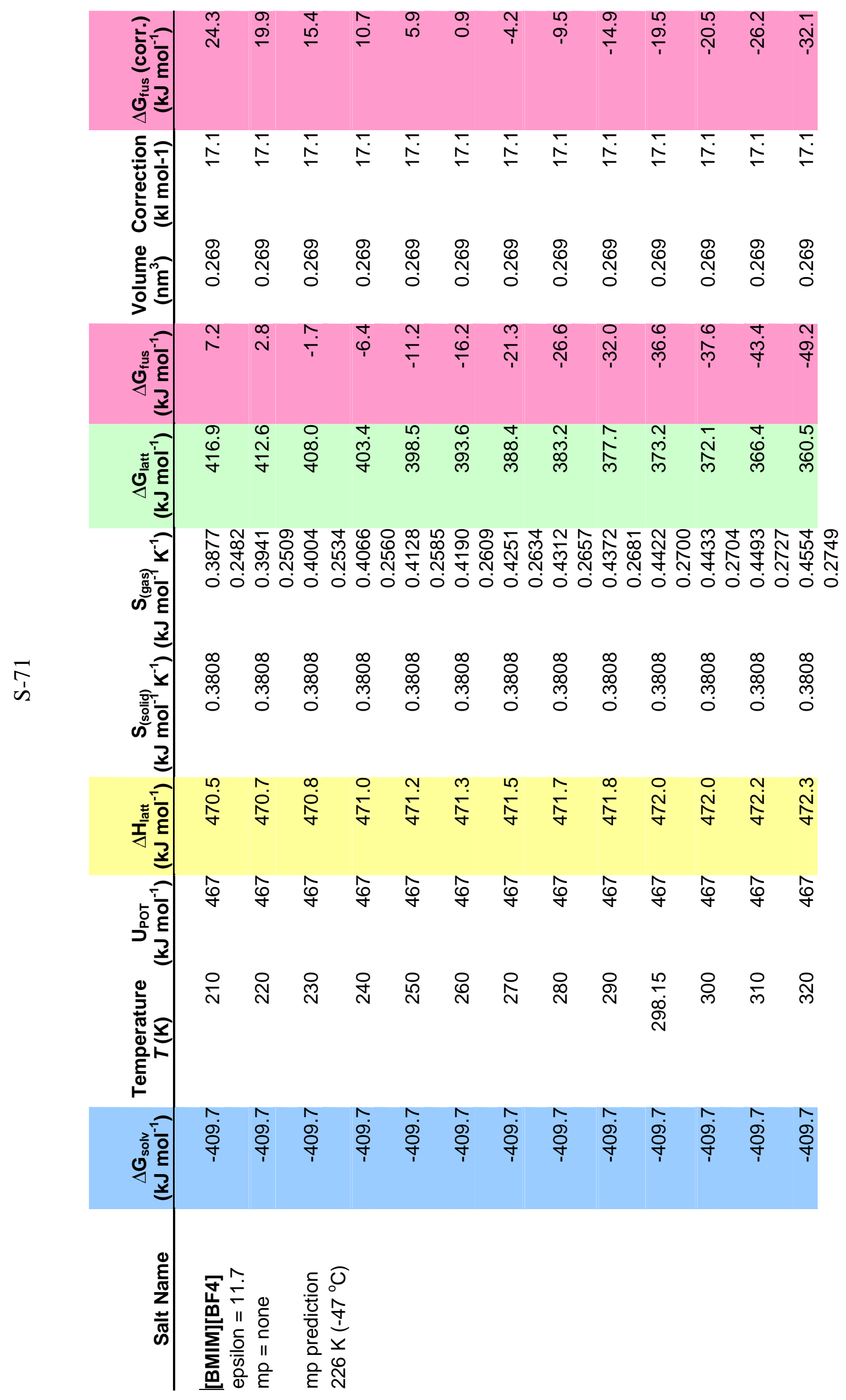




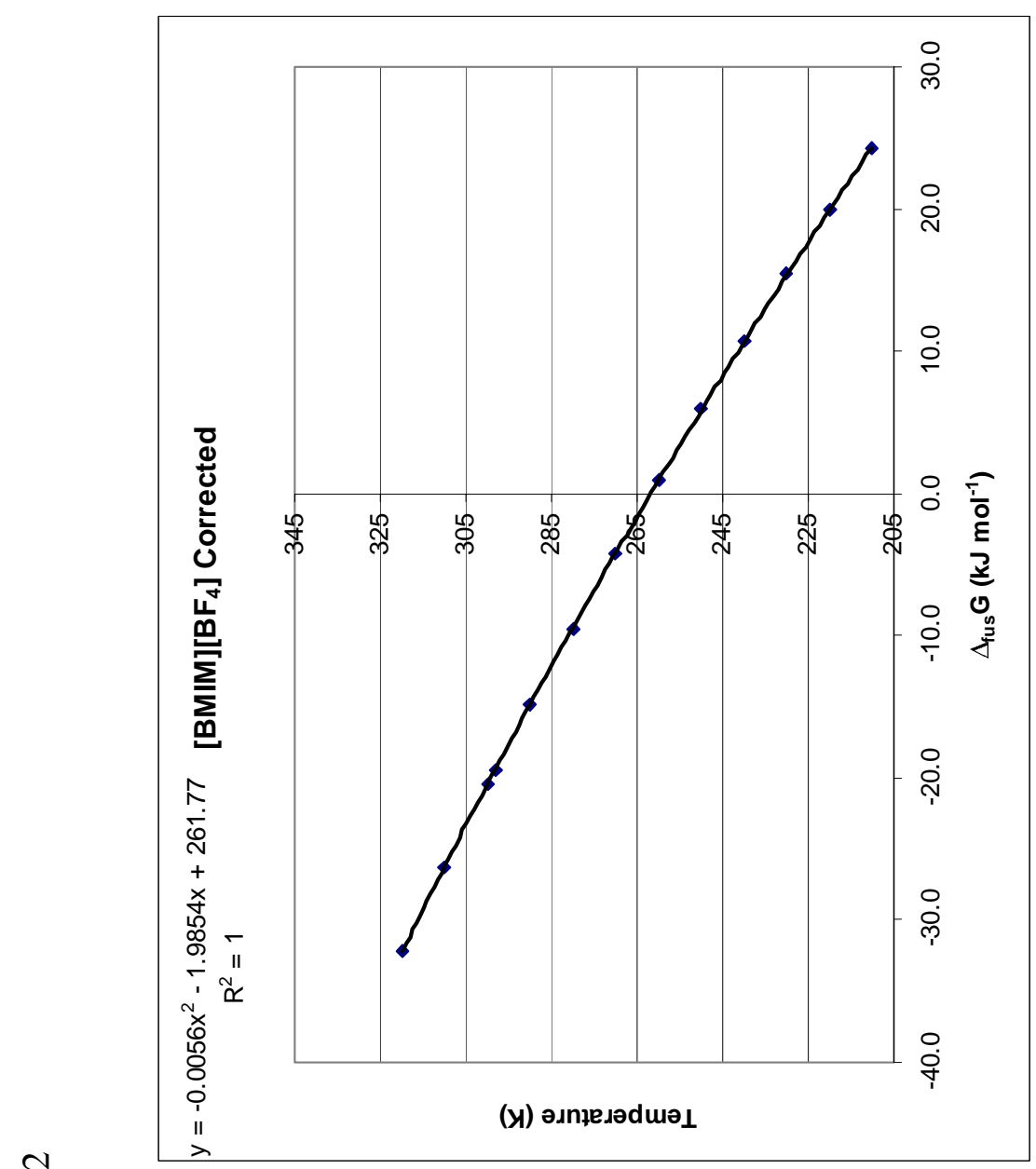

N

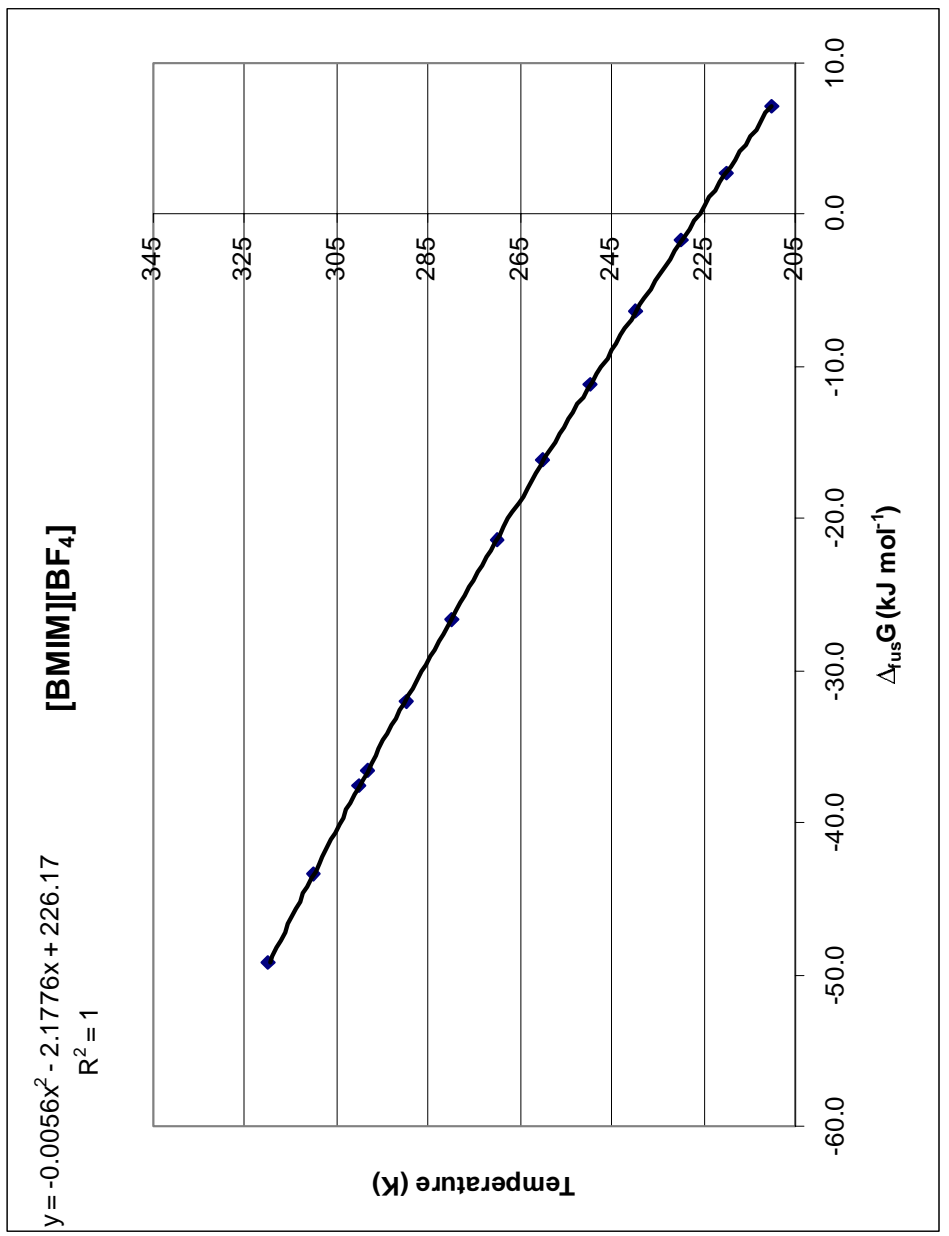




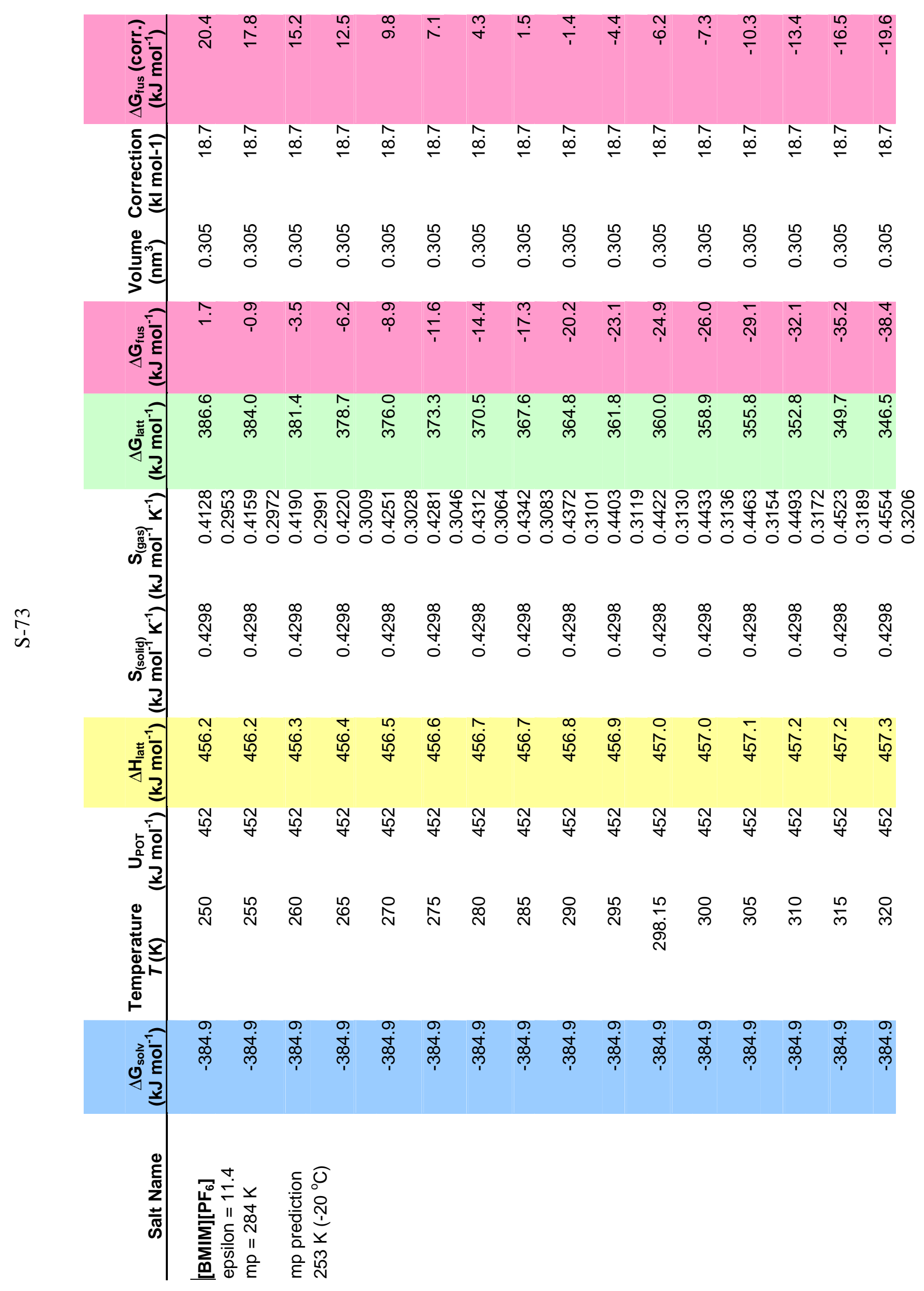




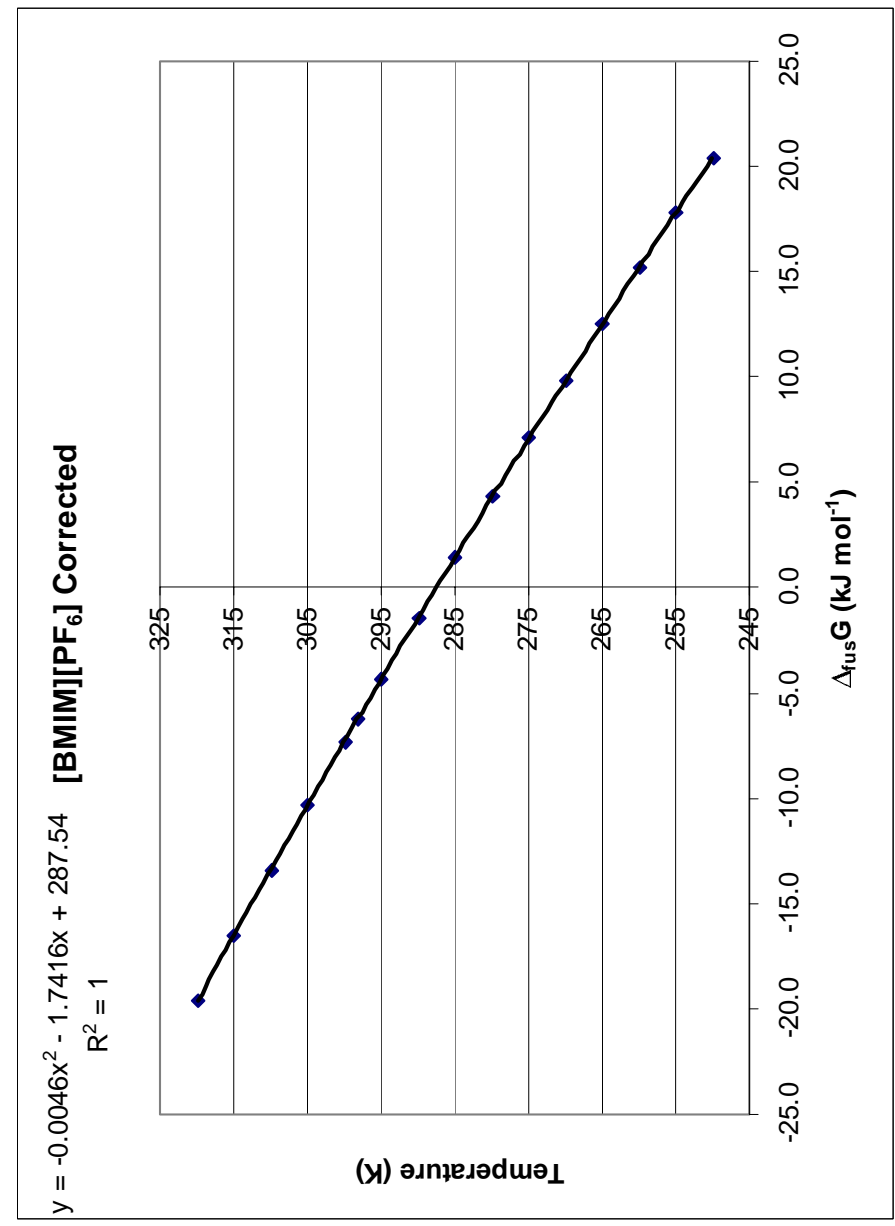

$\frac{+}{2}$

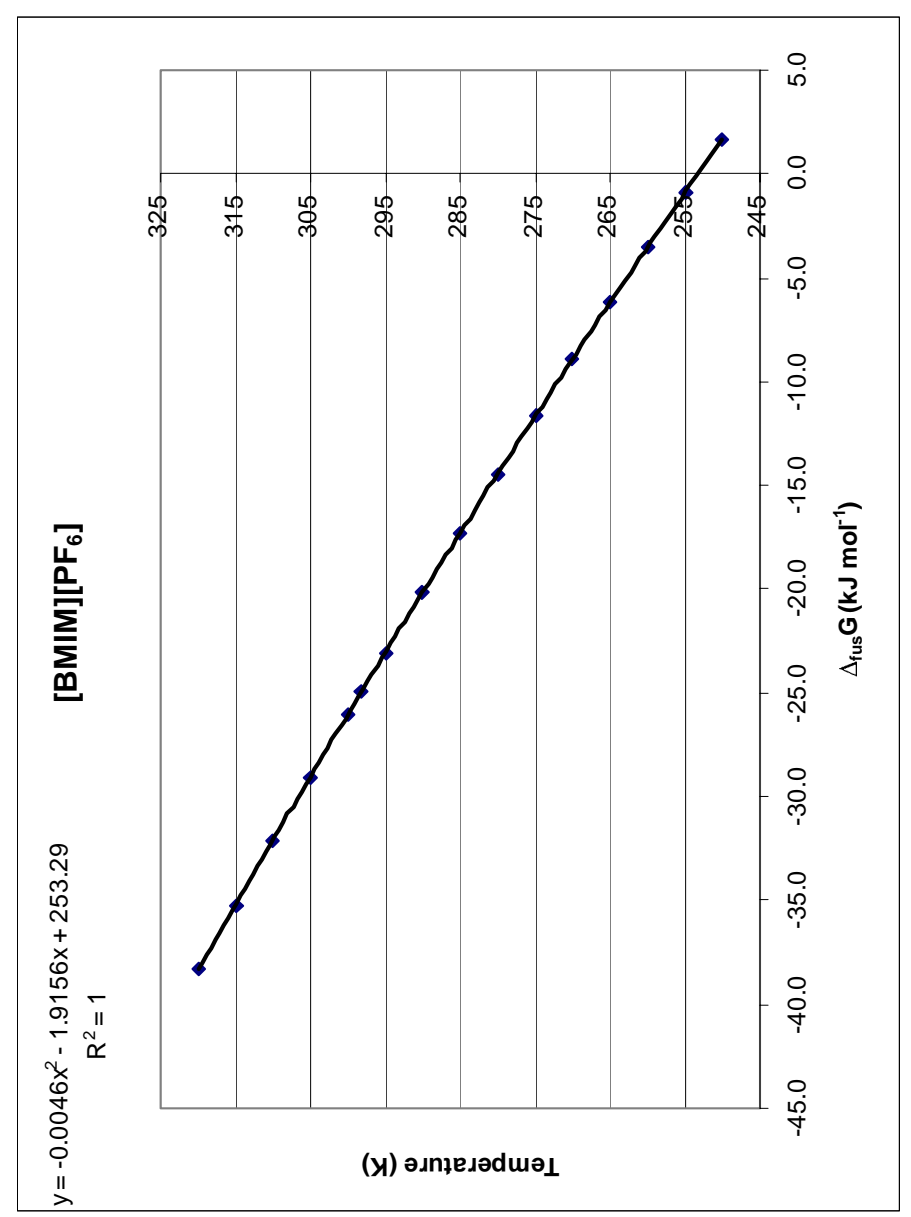




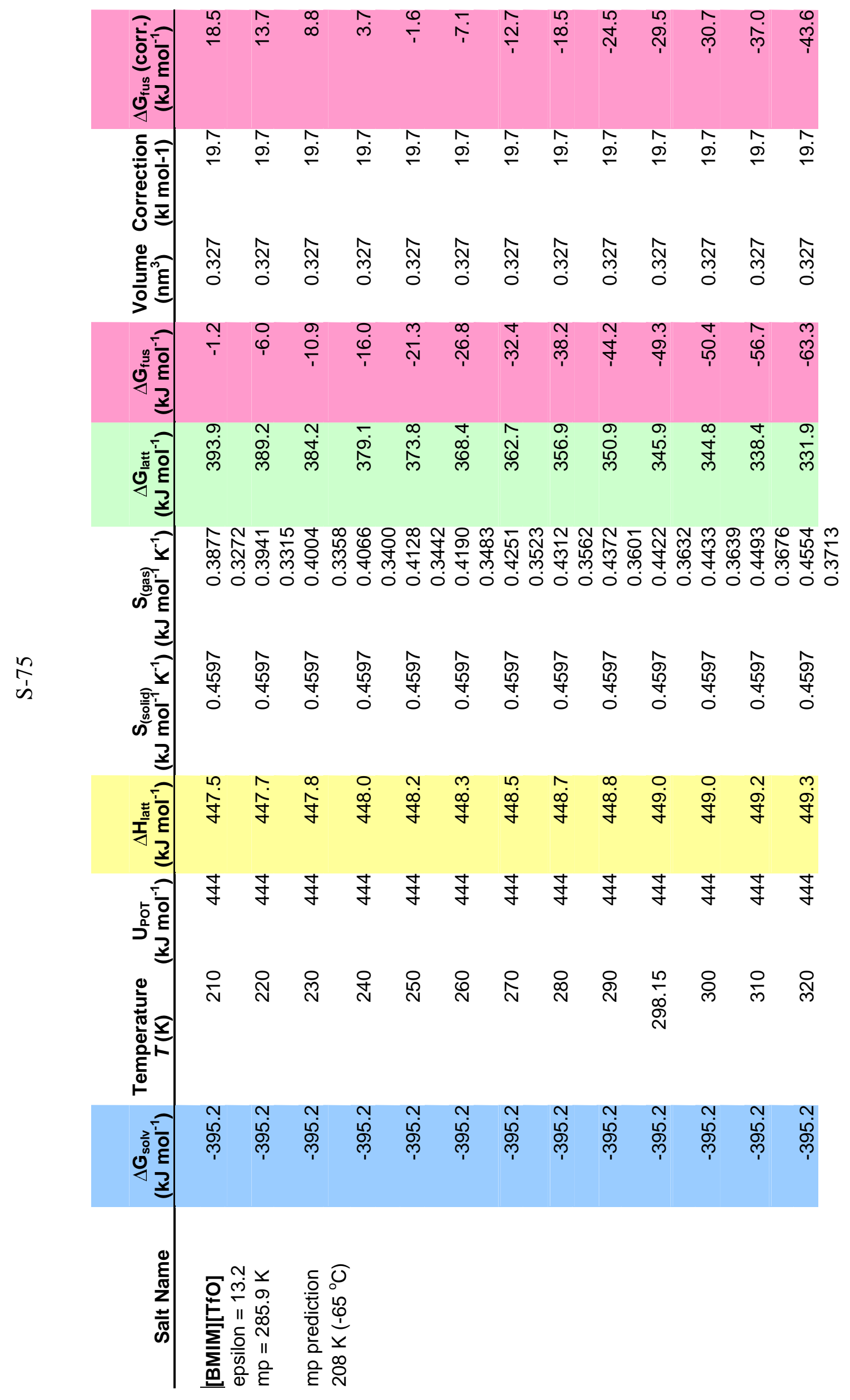




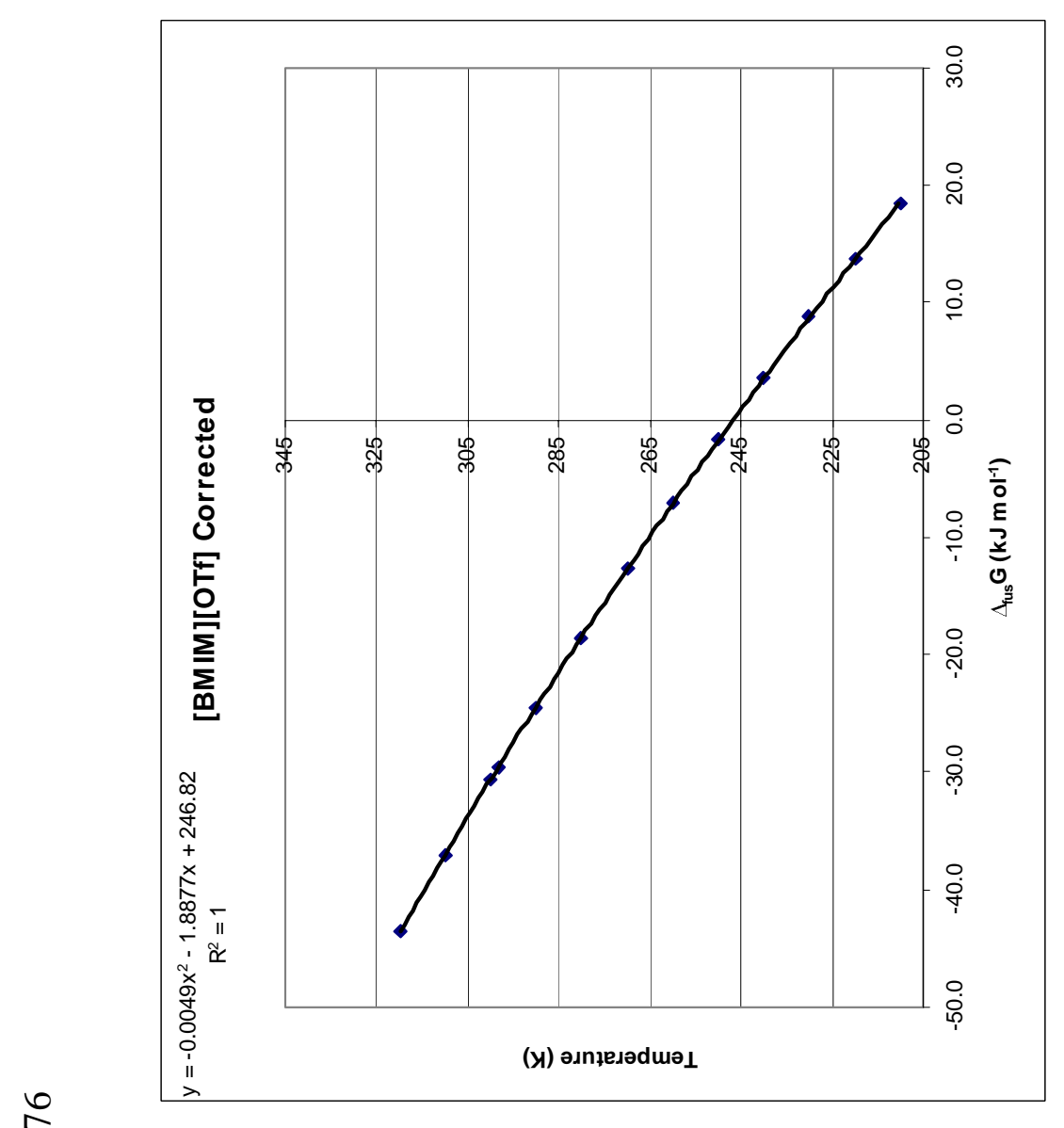

is

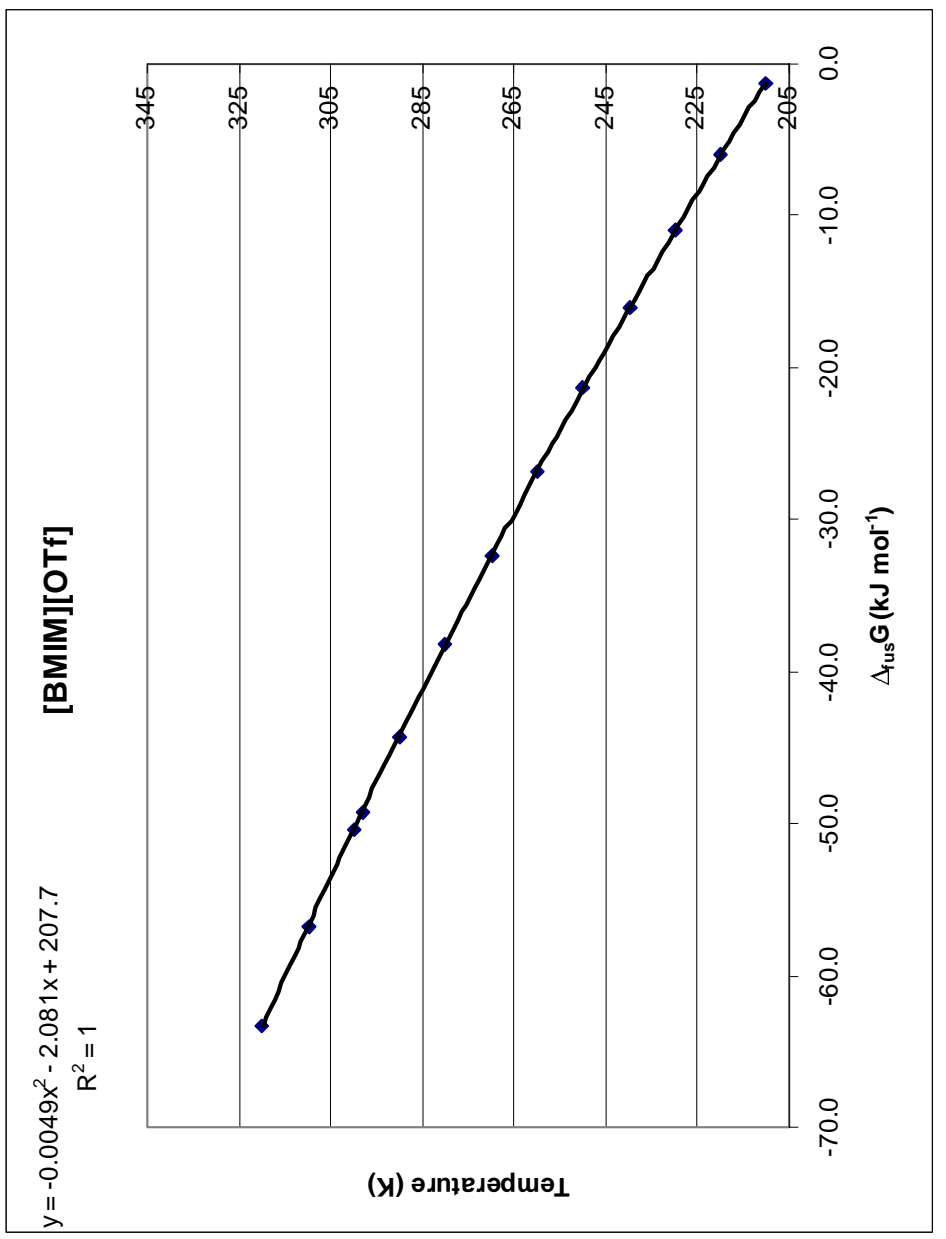




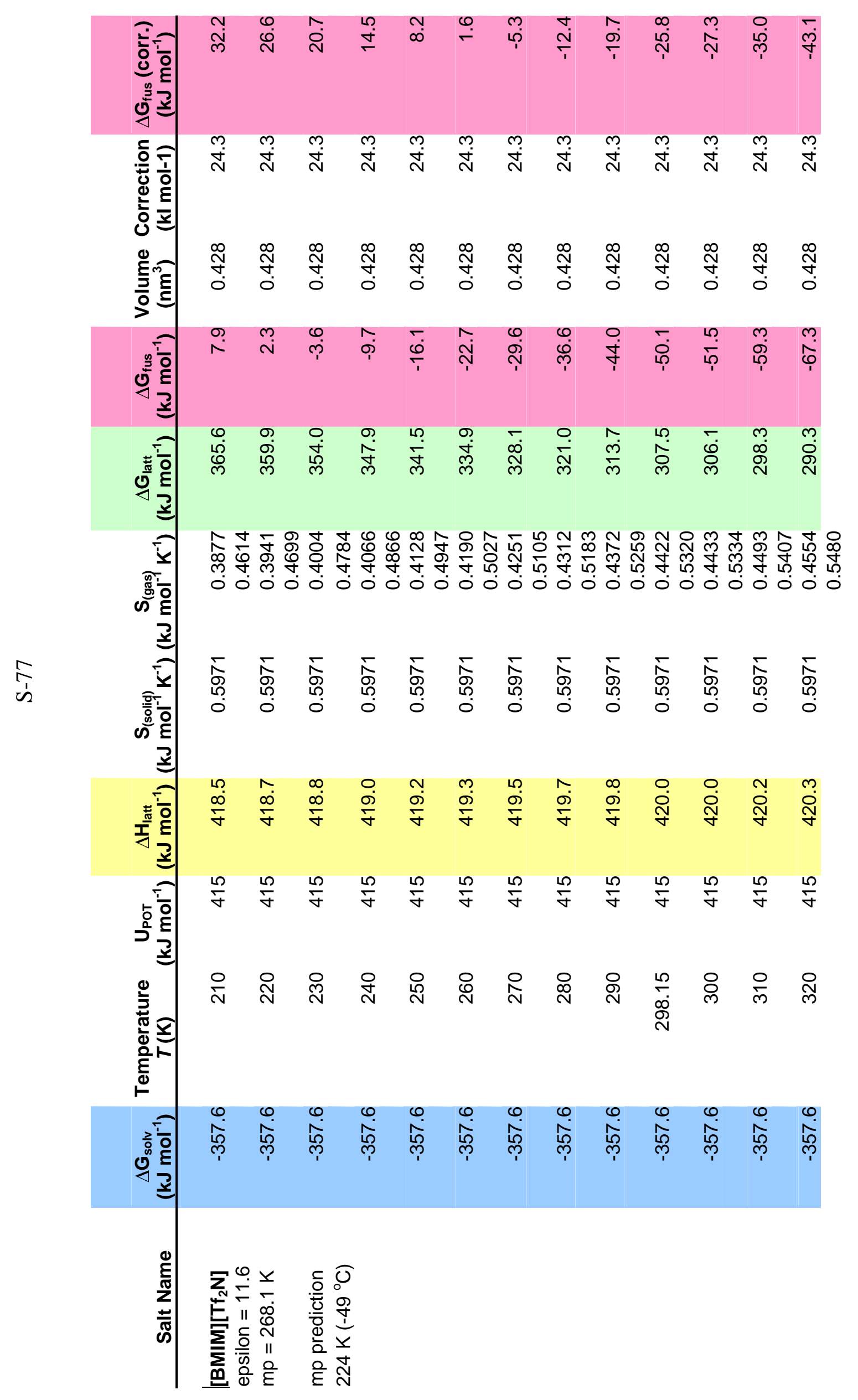



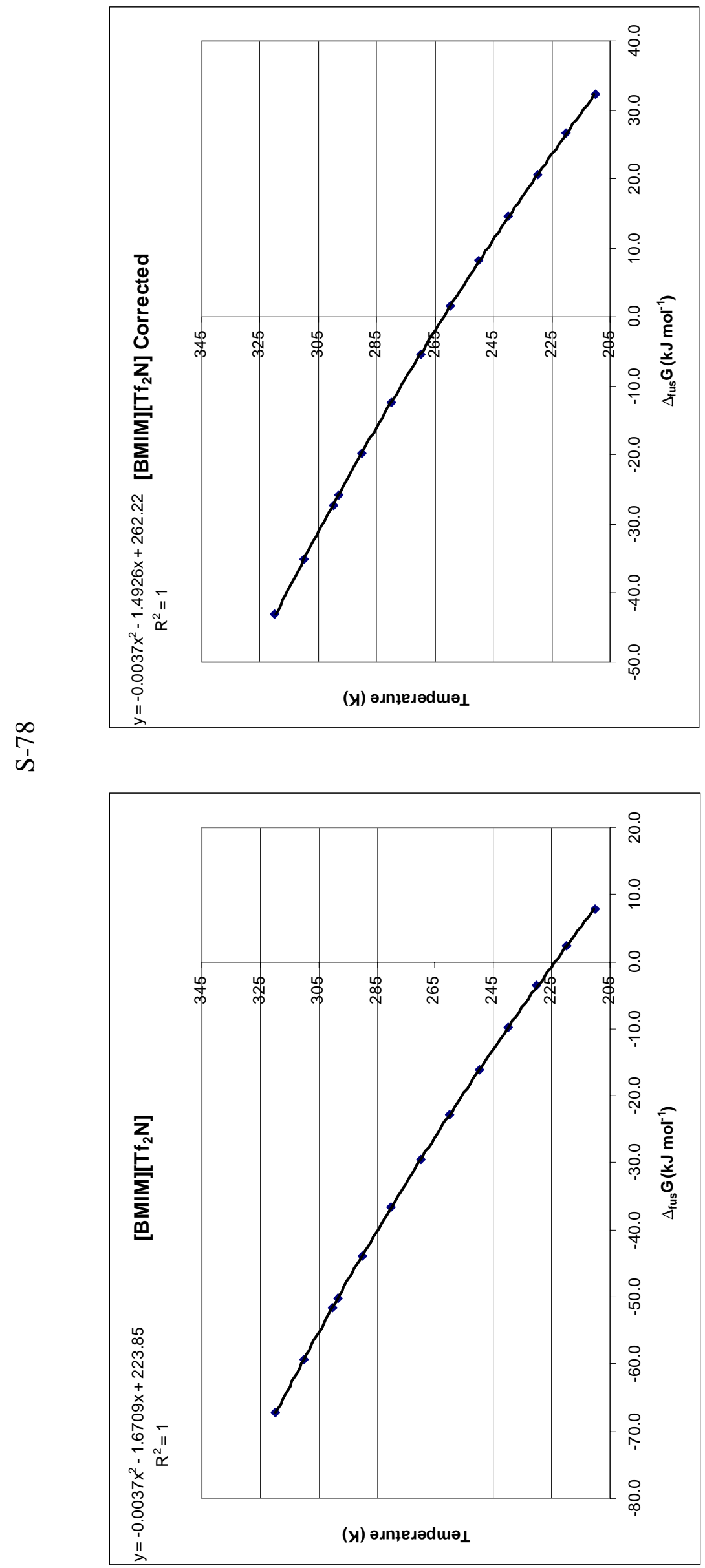


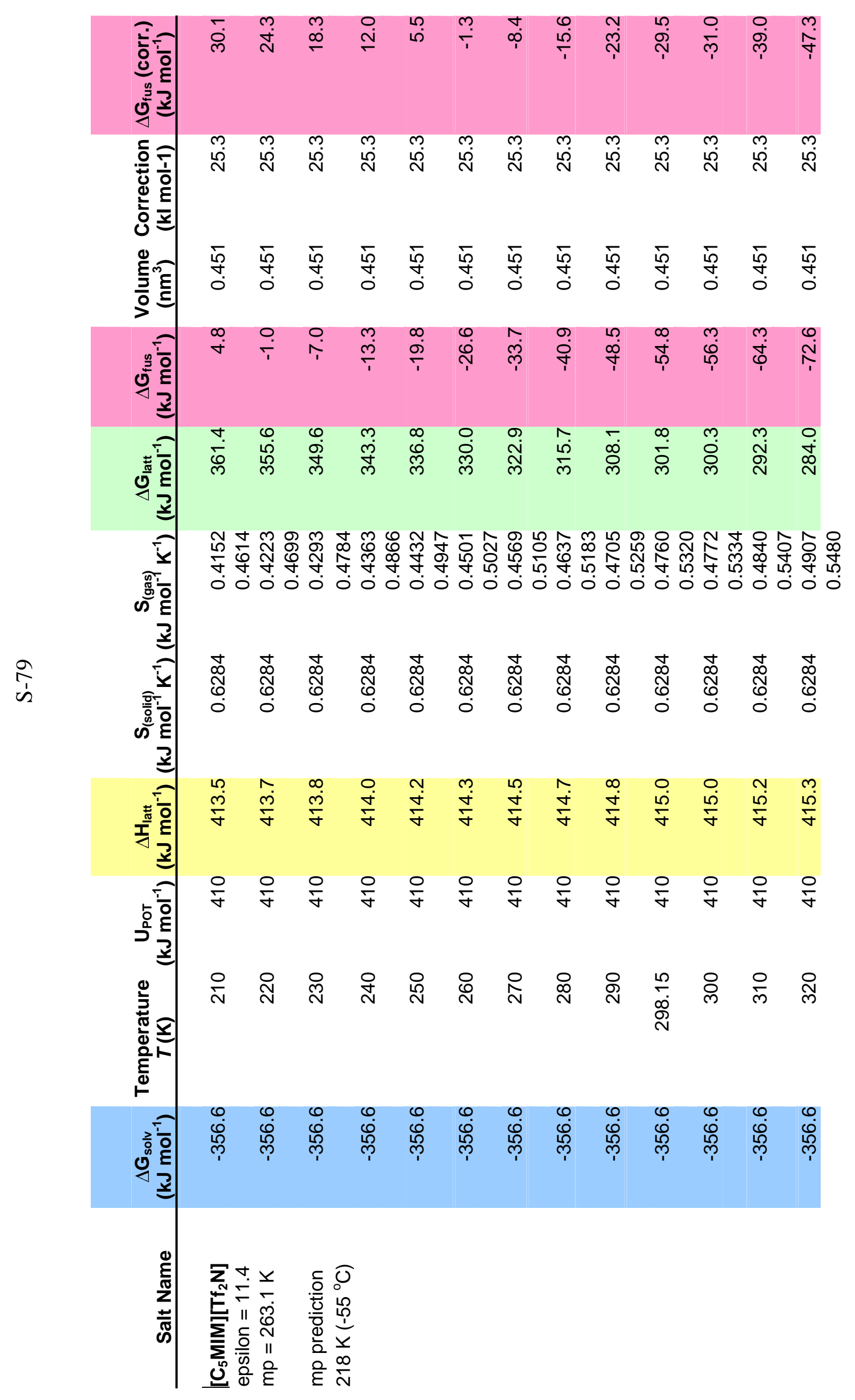




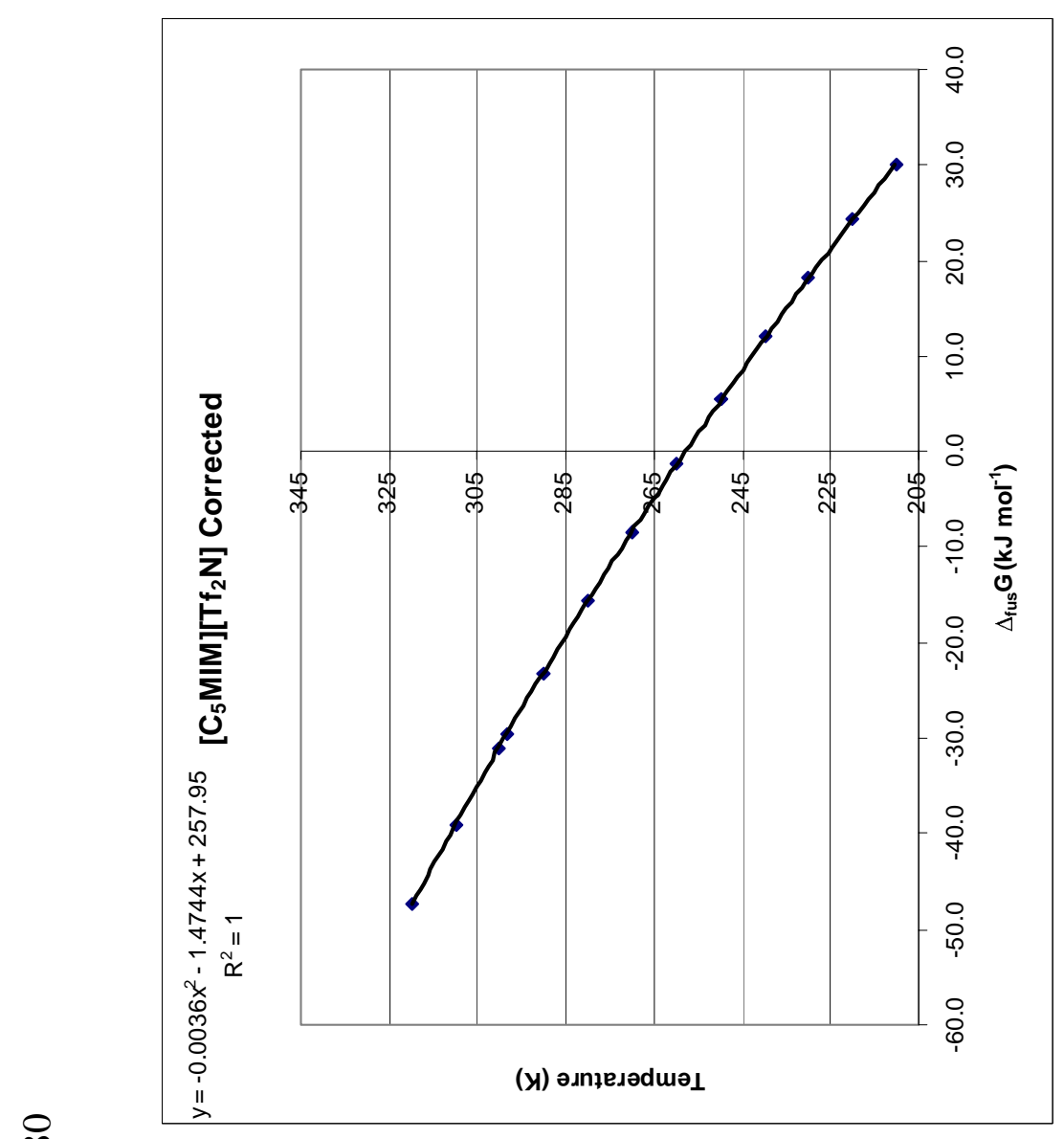

$\stackrel{1}{\infty}$
1
1

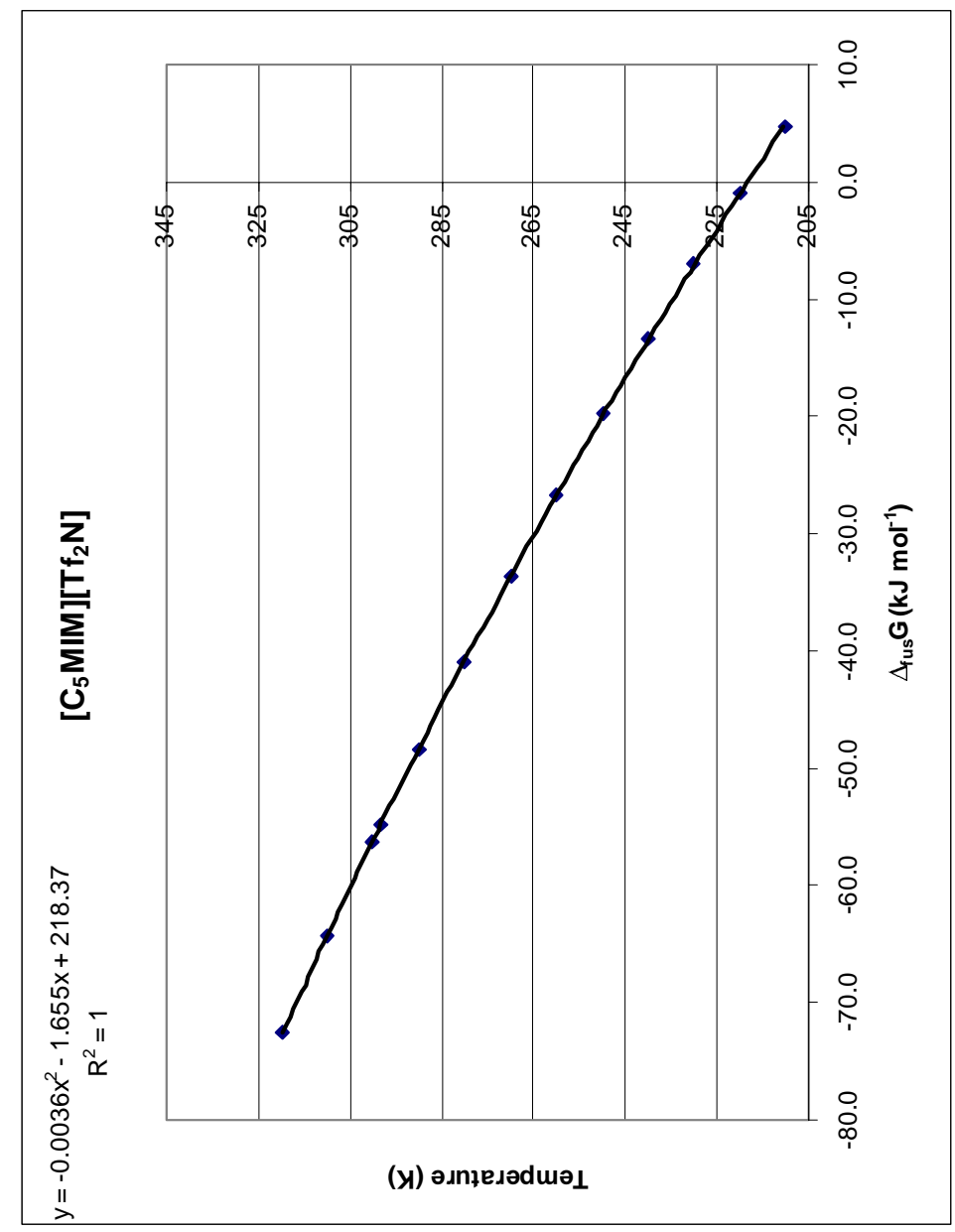




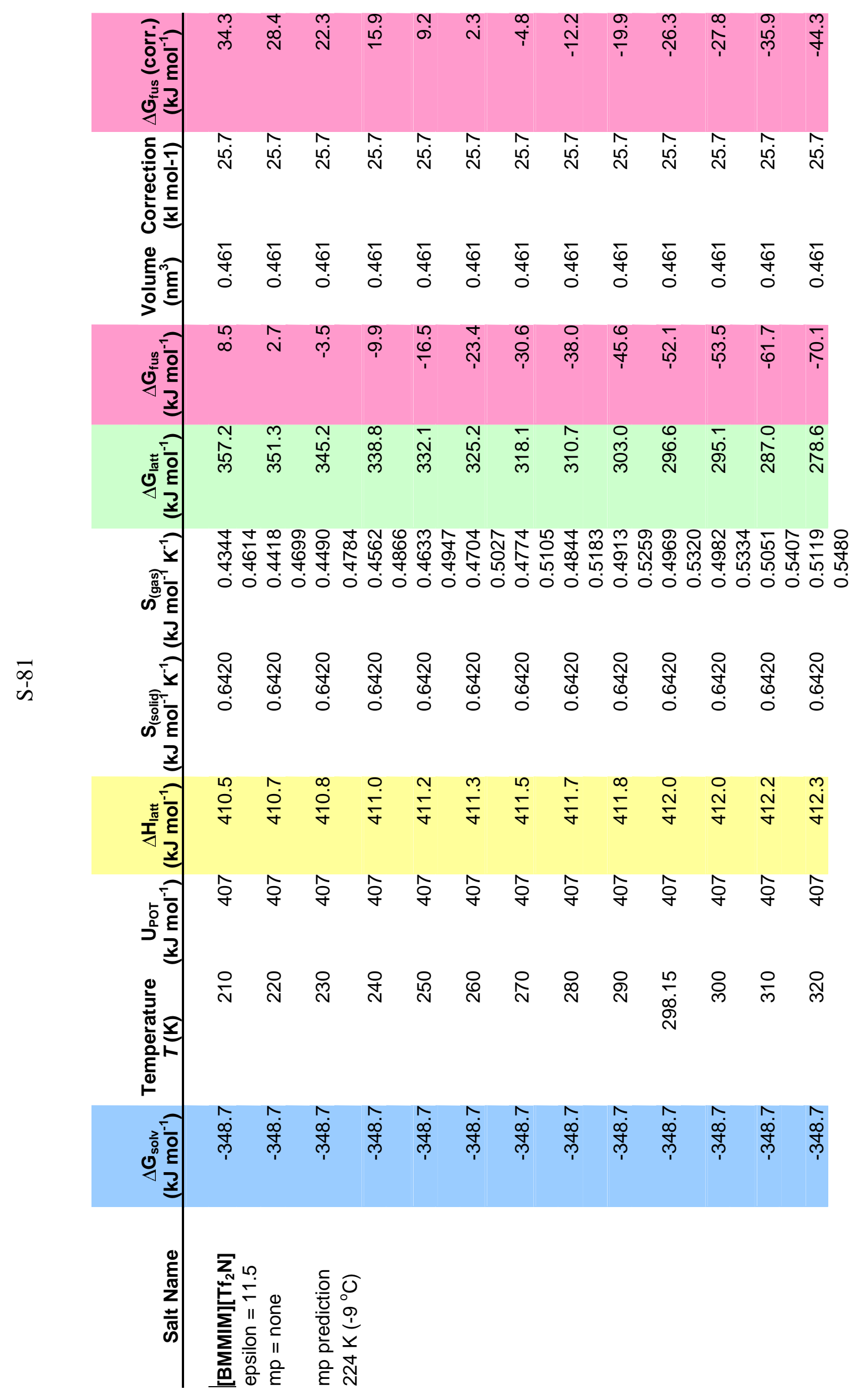


N
1
í
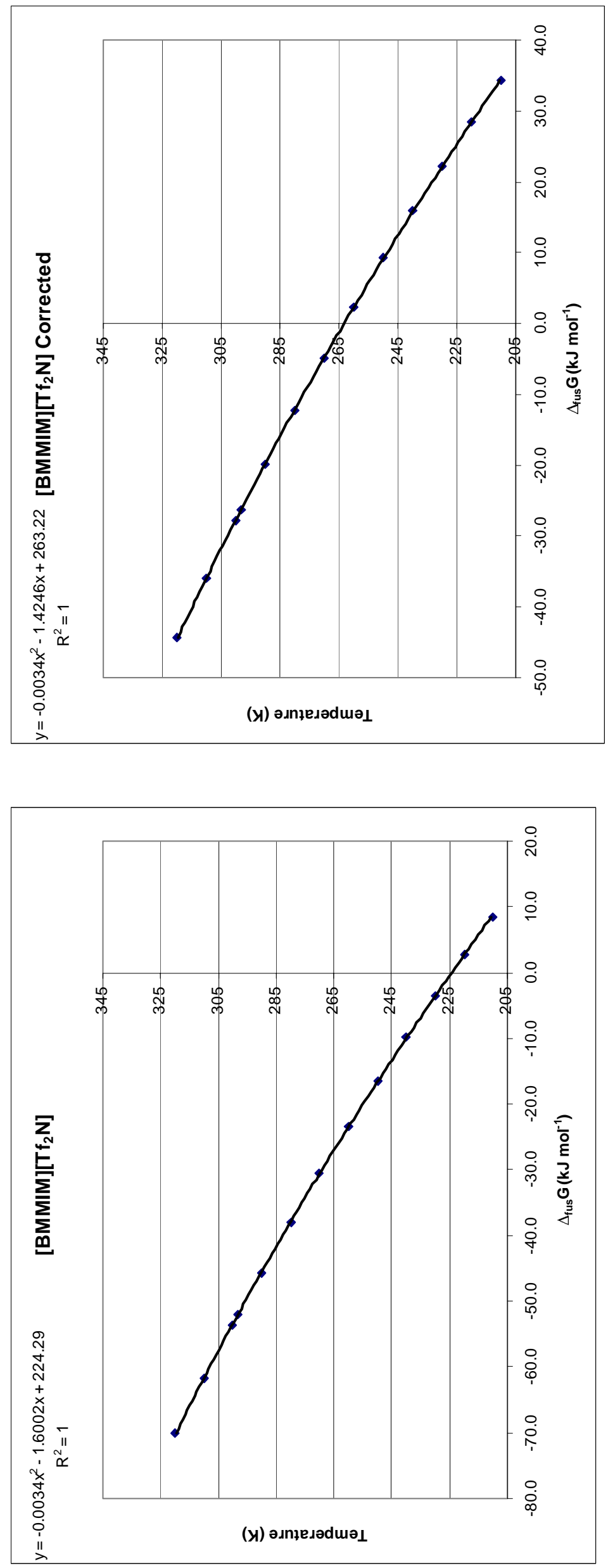


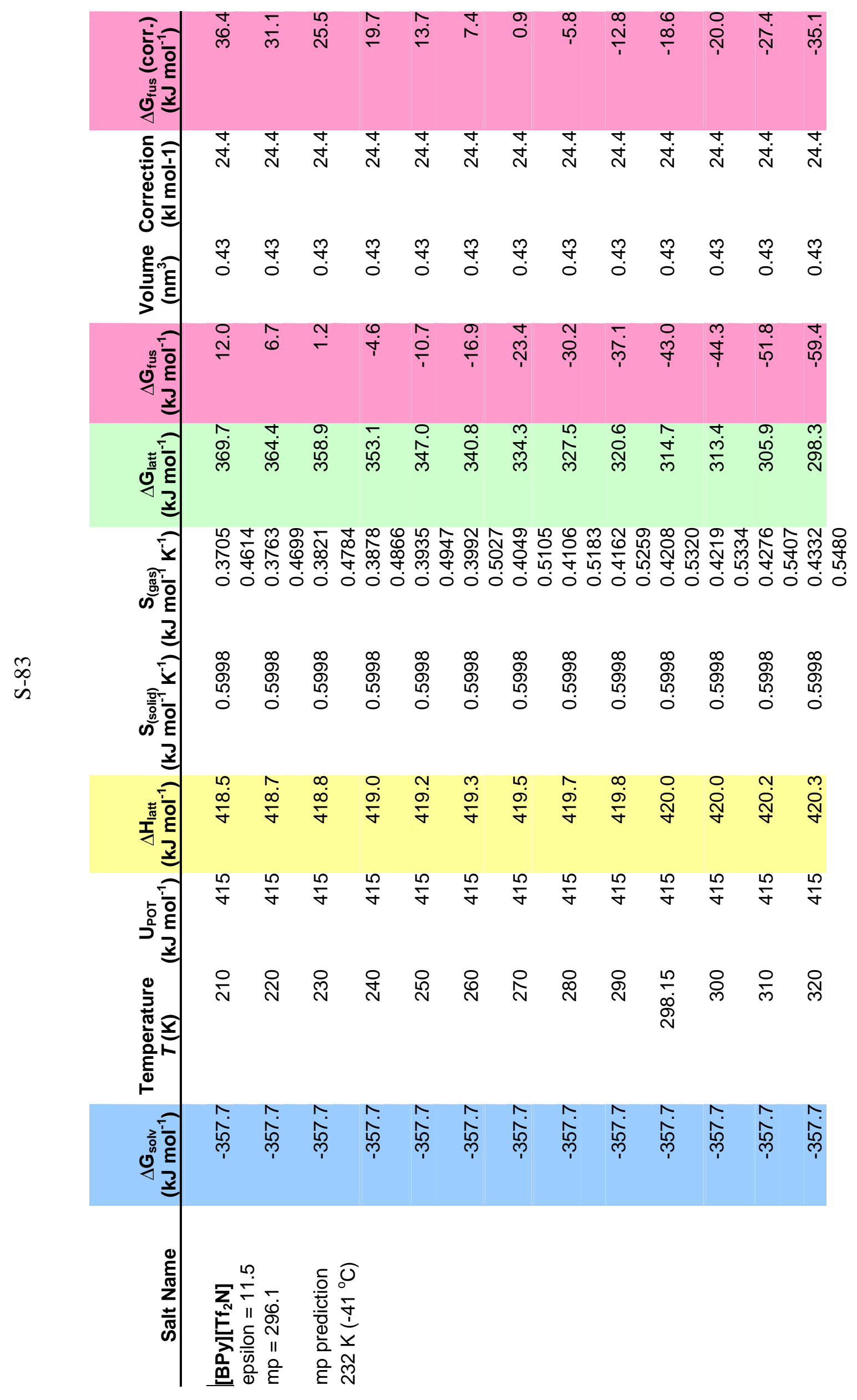




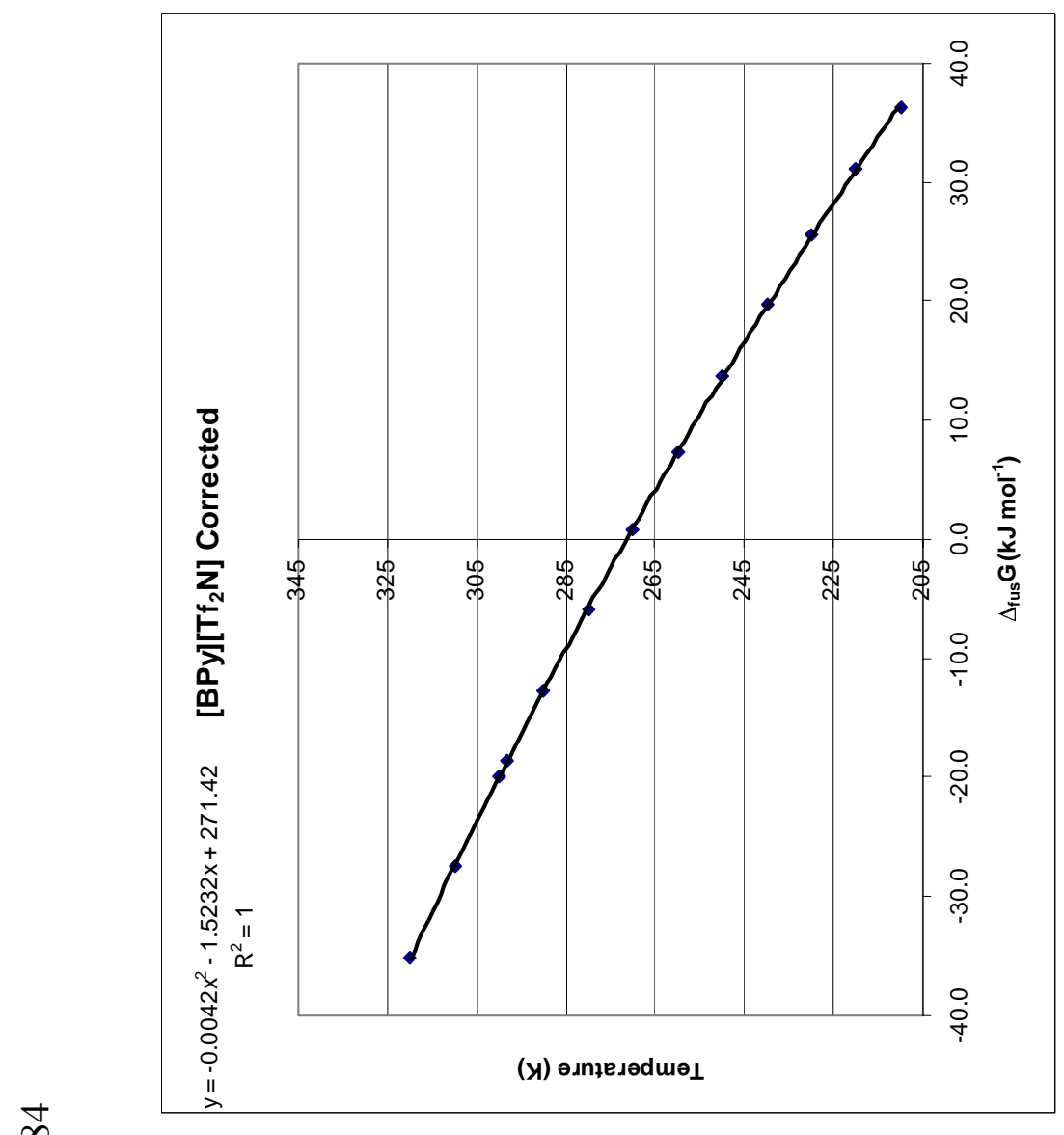

i

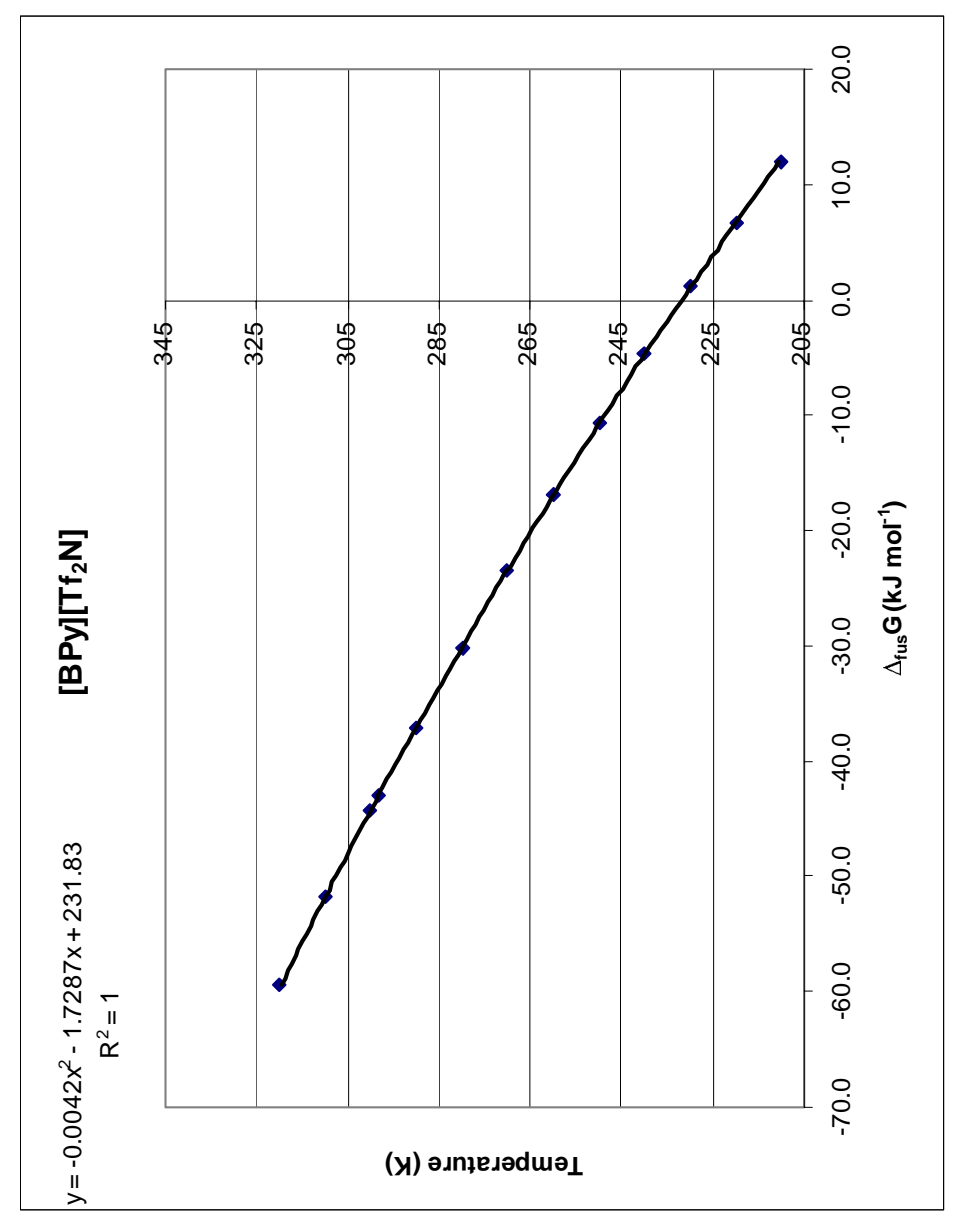




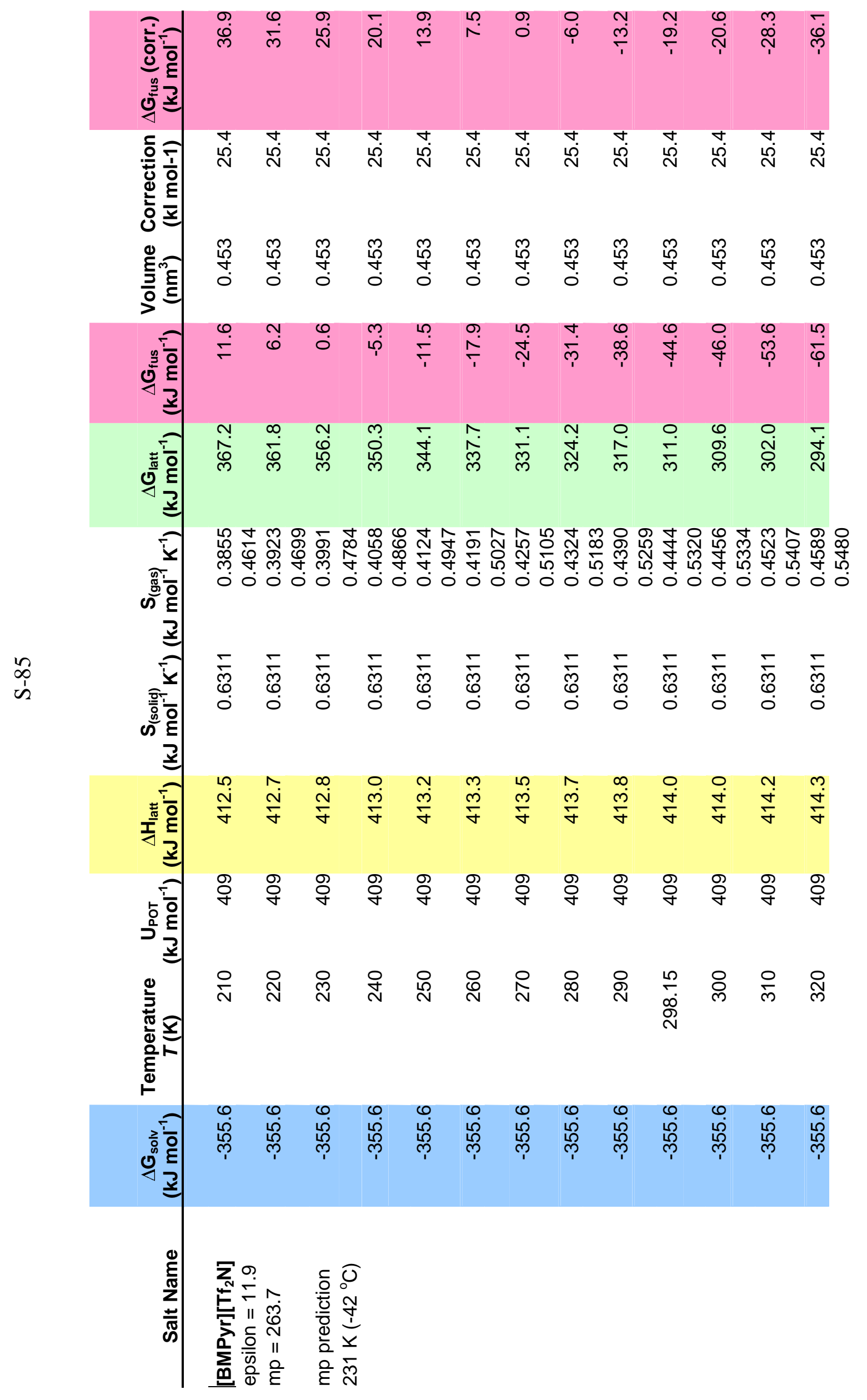




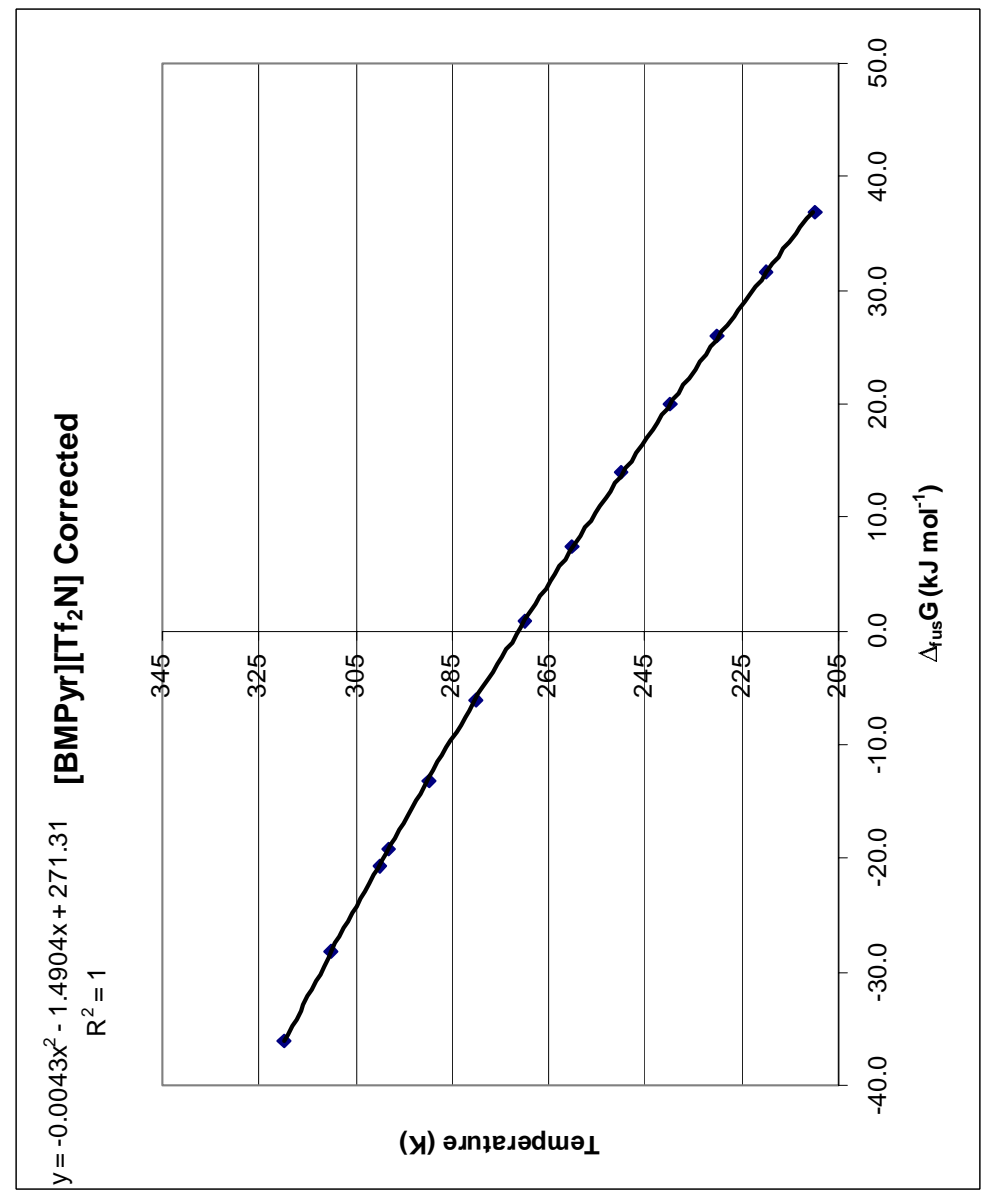

0
$\infty$
1

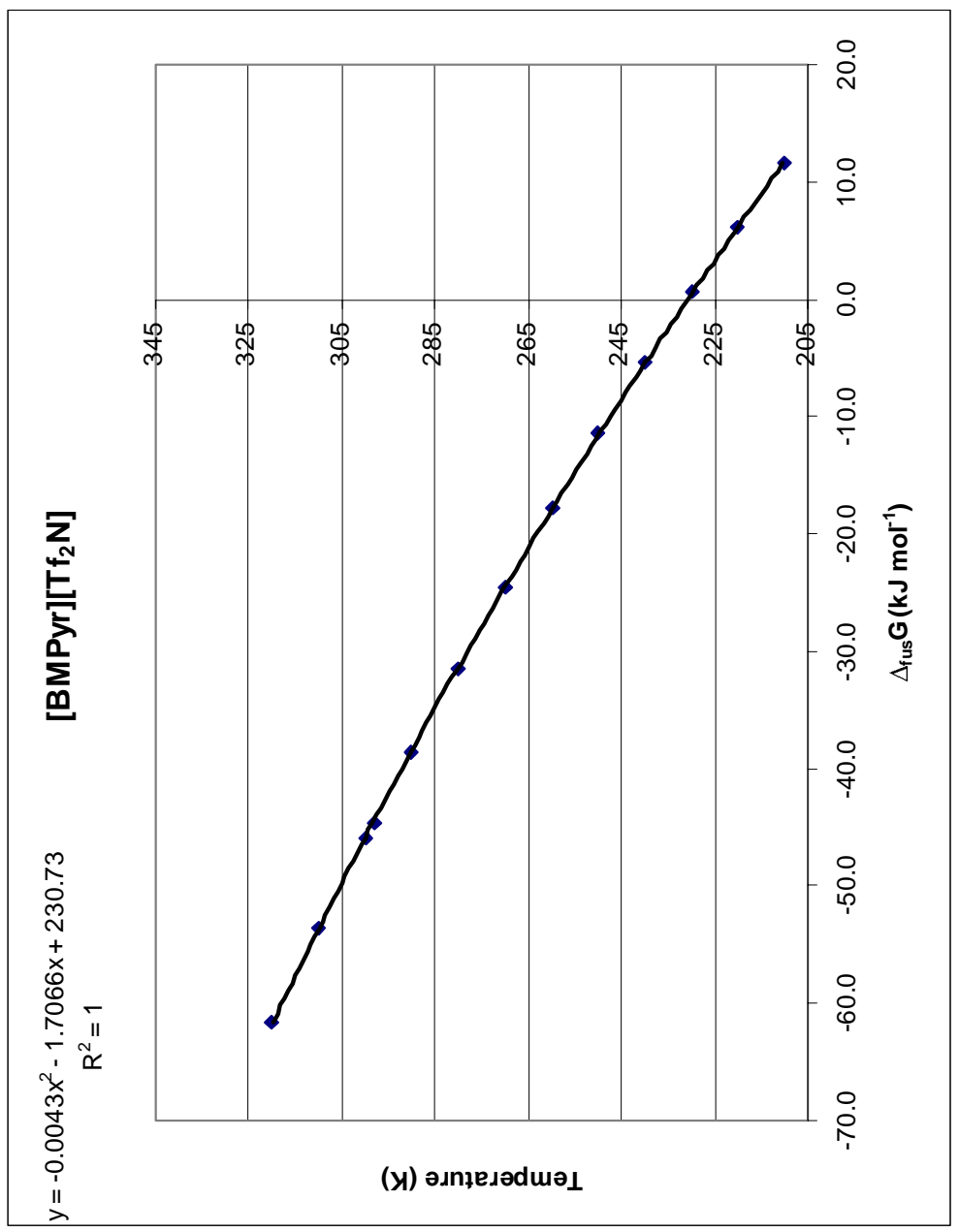




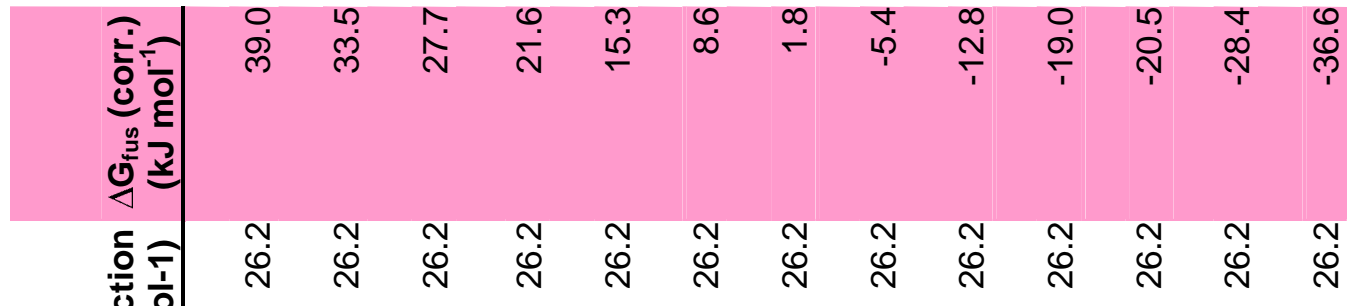

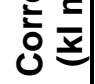

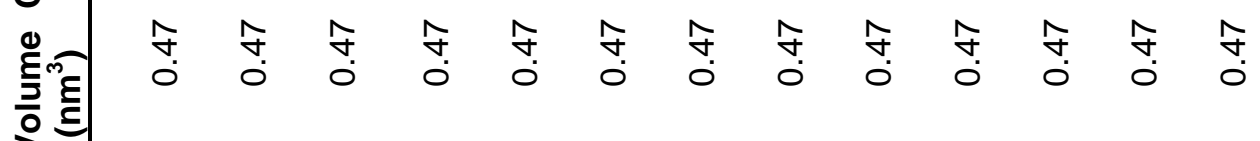

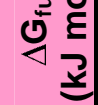

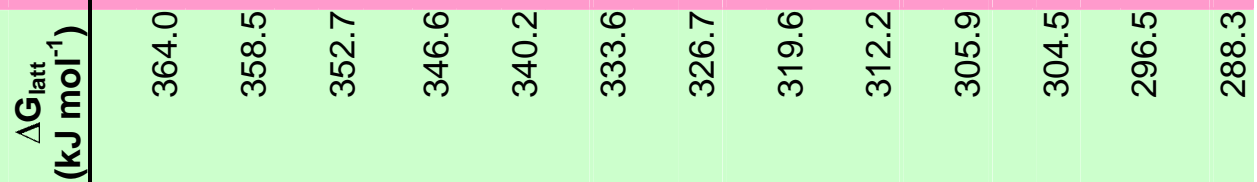

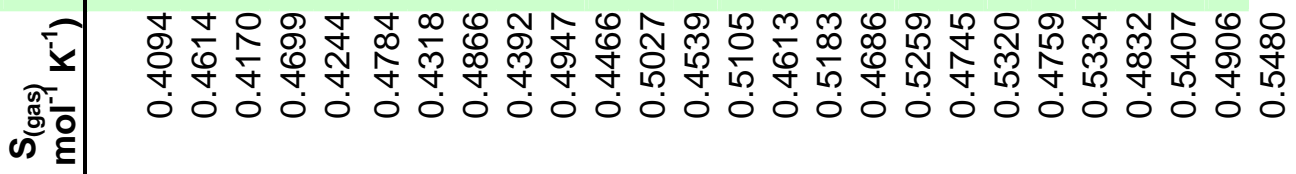

$$
\begin{aligned}
& \infty
\end{aligned}
$$

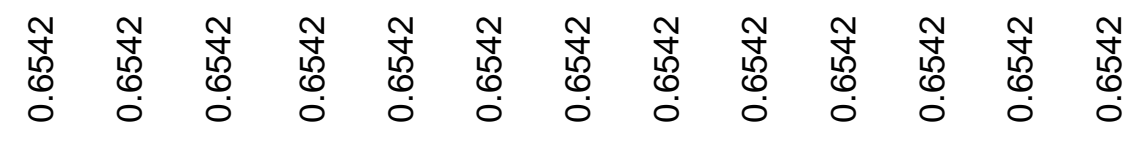

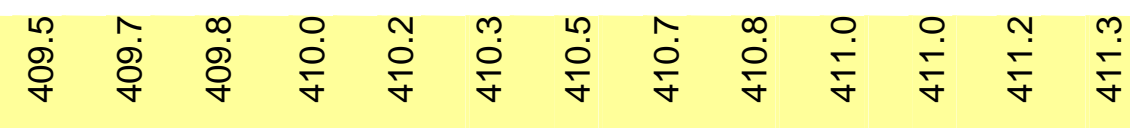

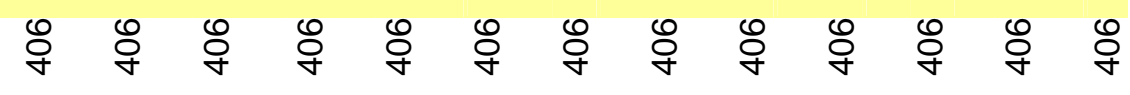

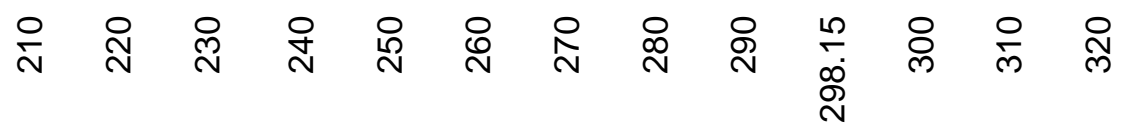




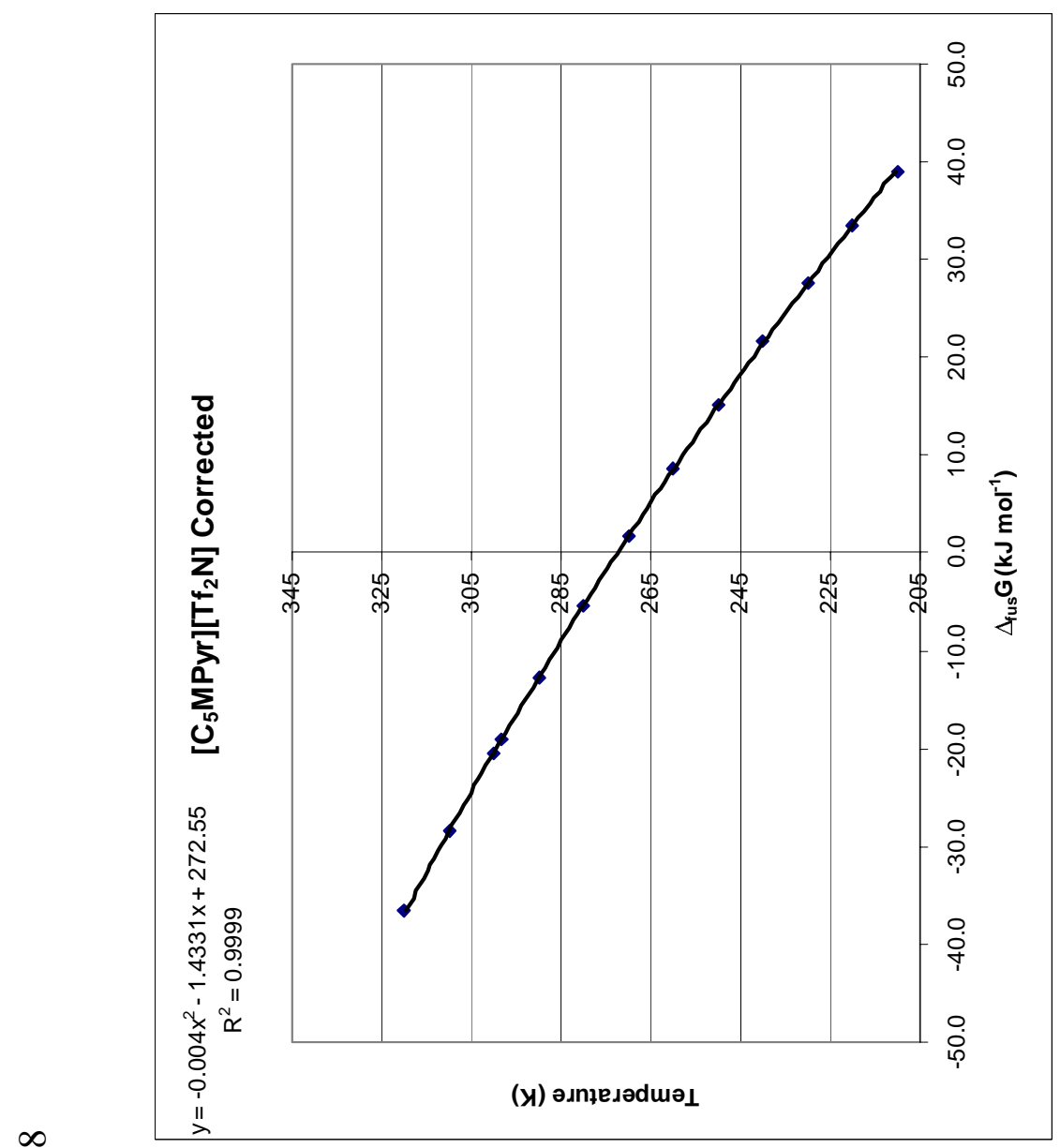

$\infty$
$\infty$
1

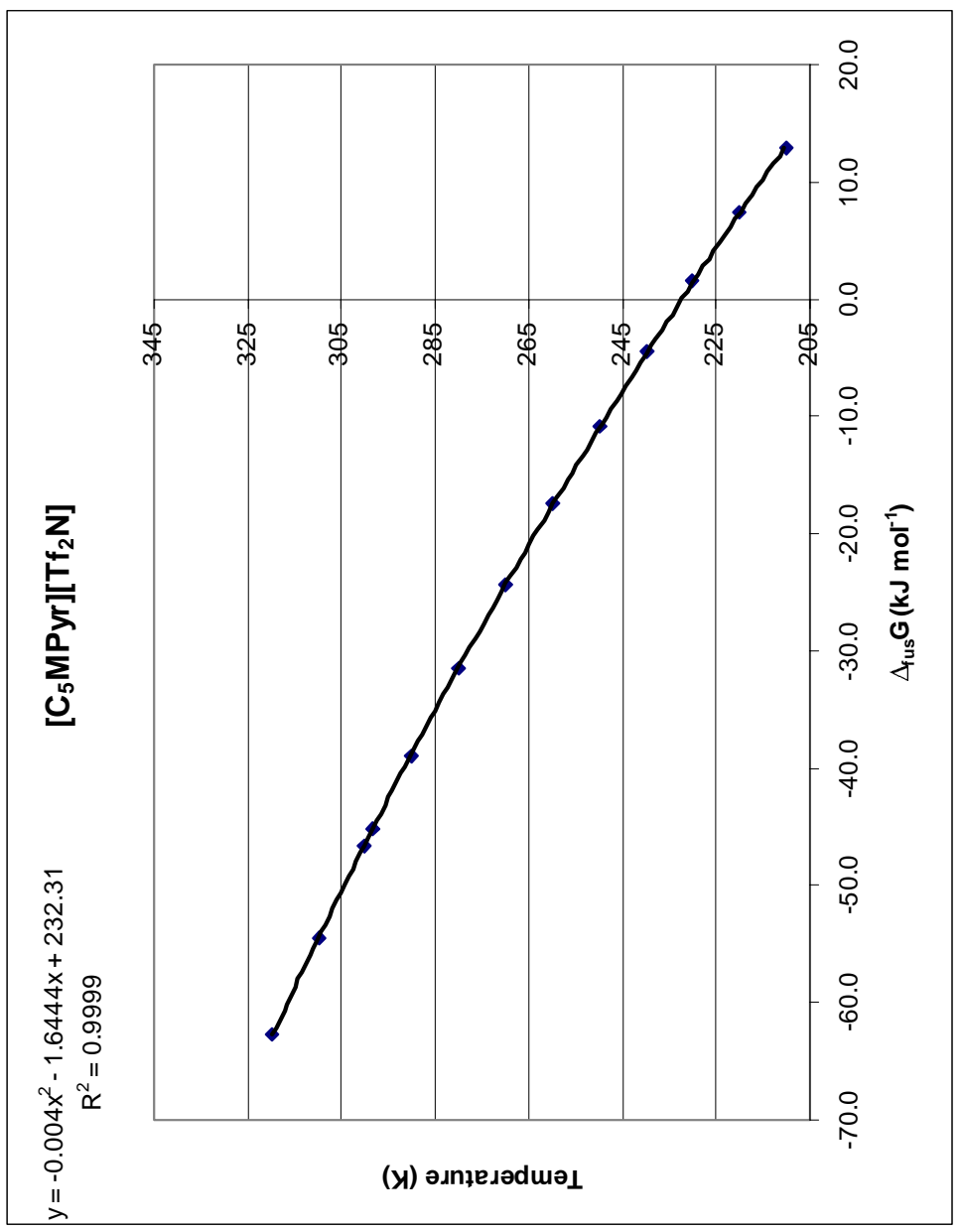




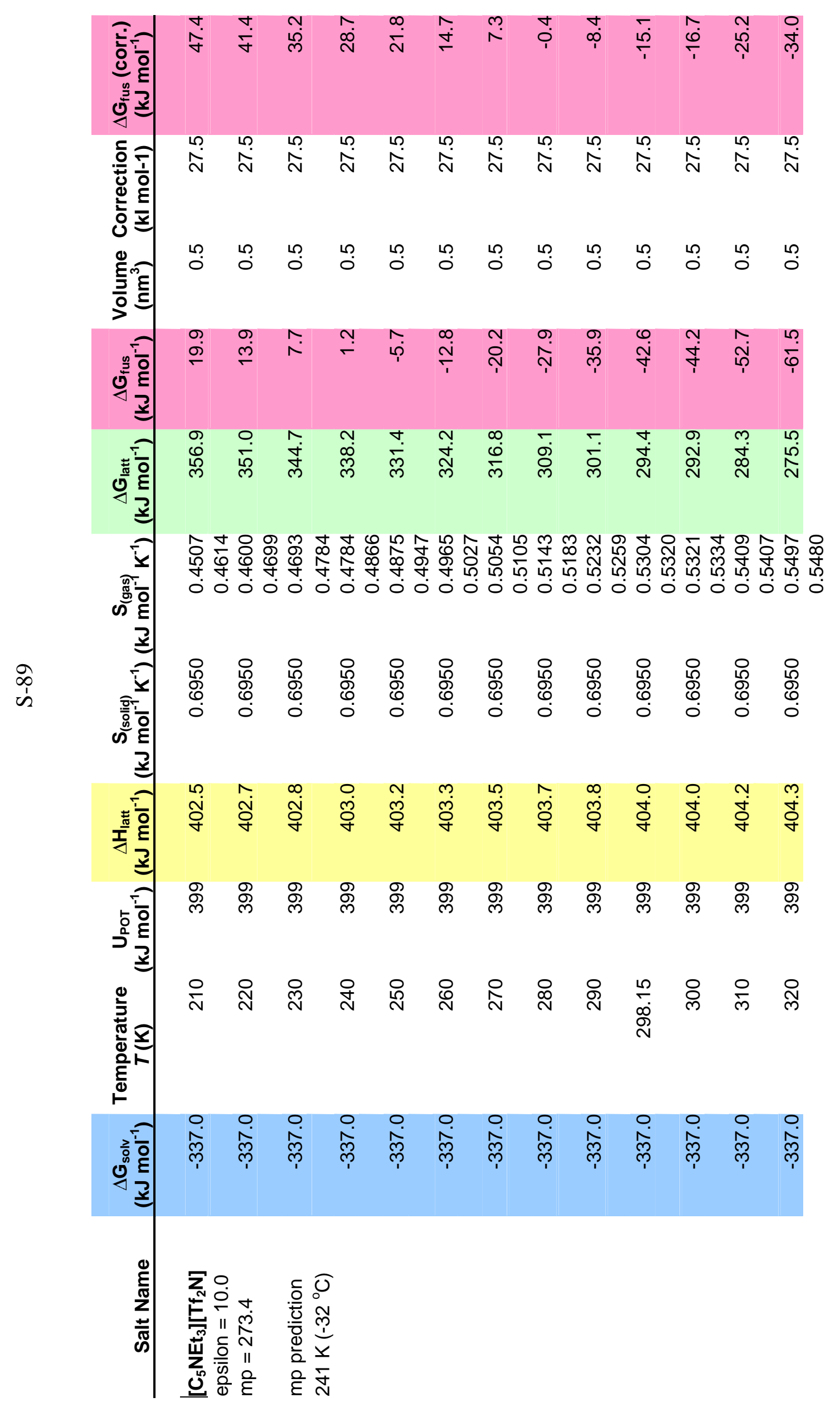




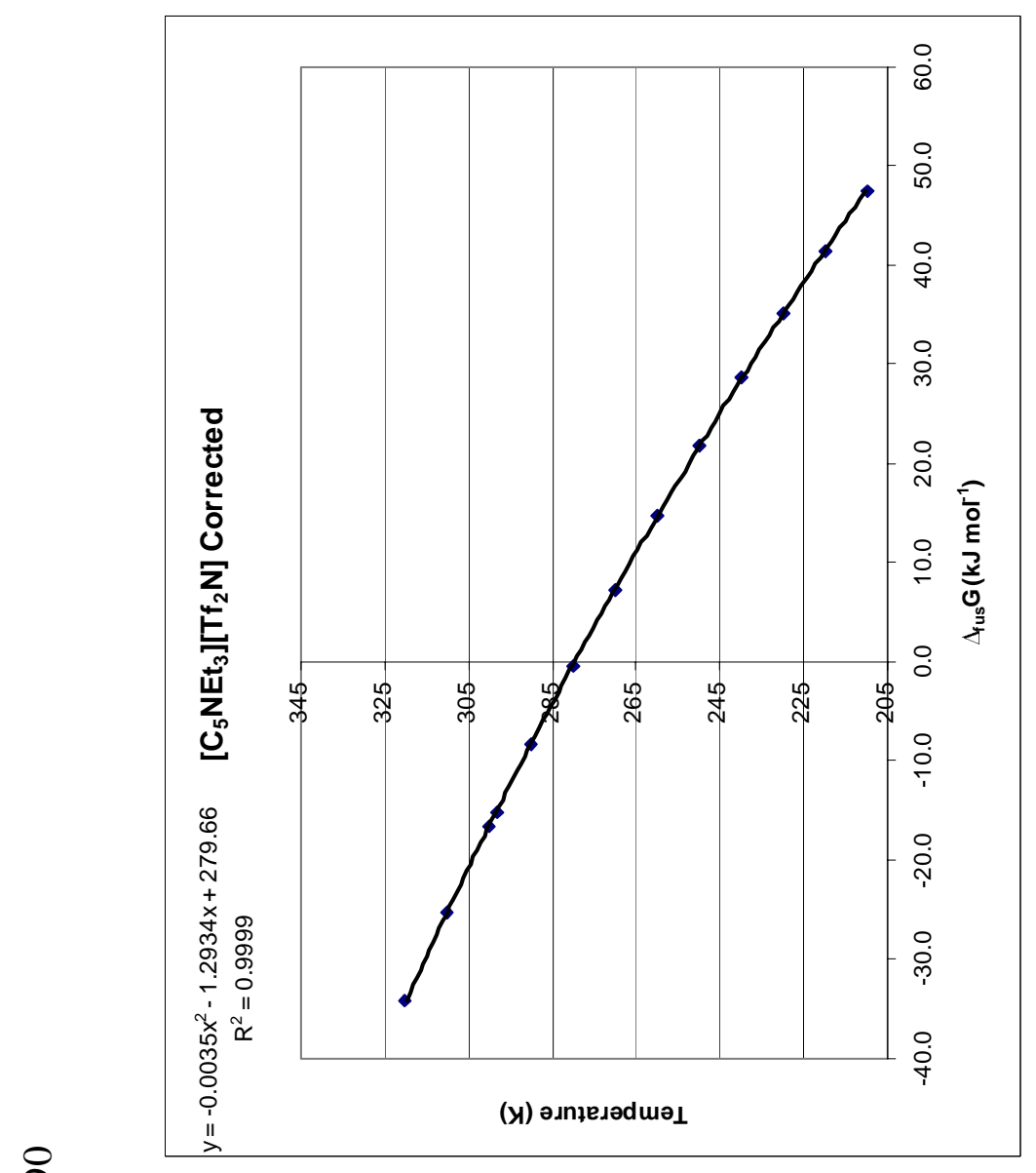

ळ

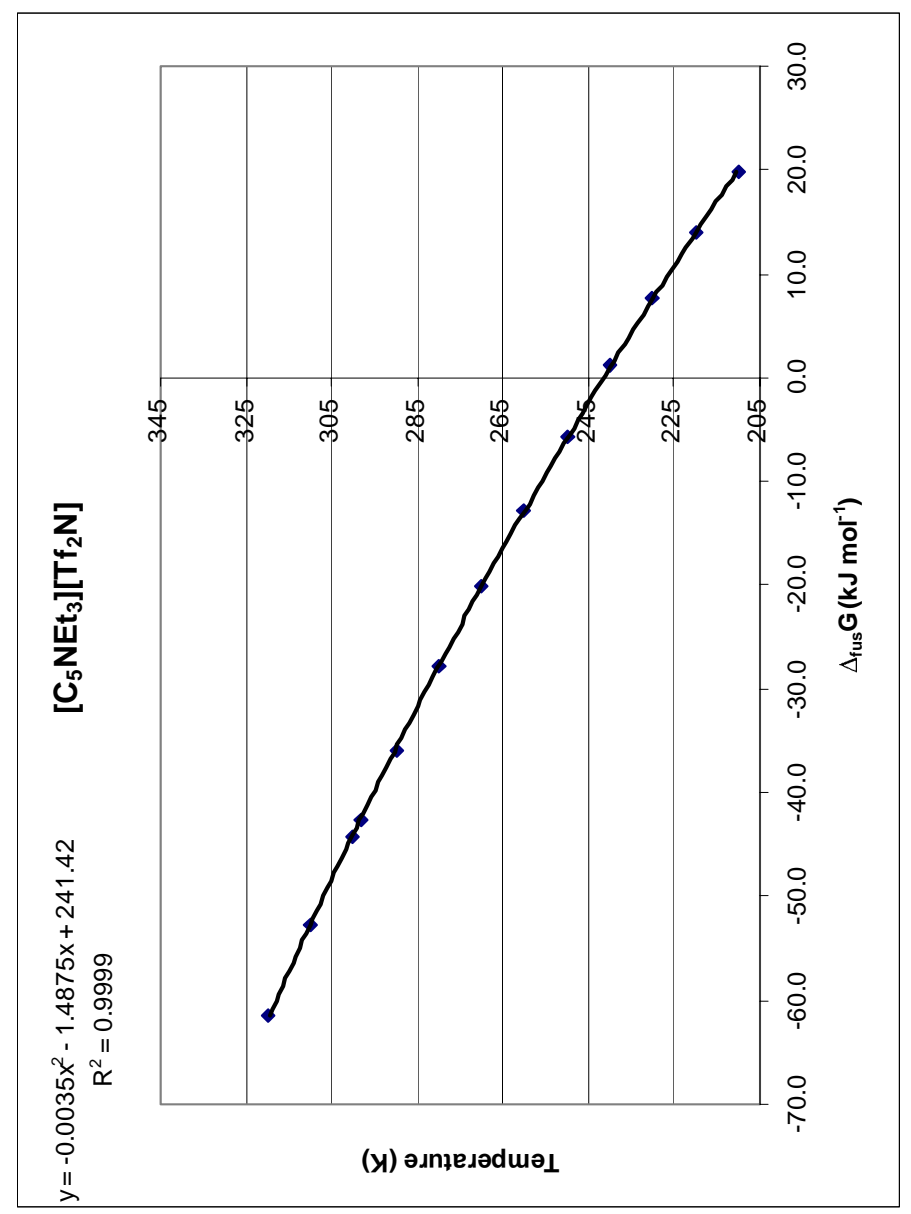




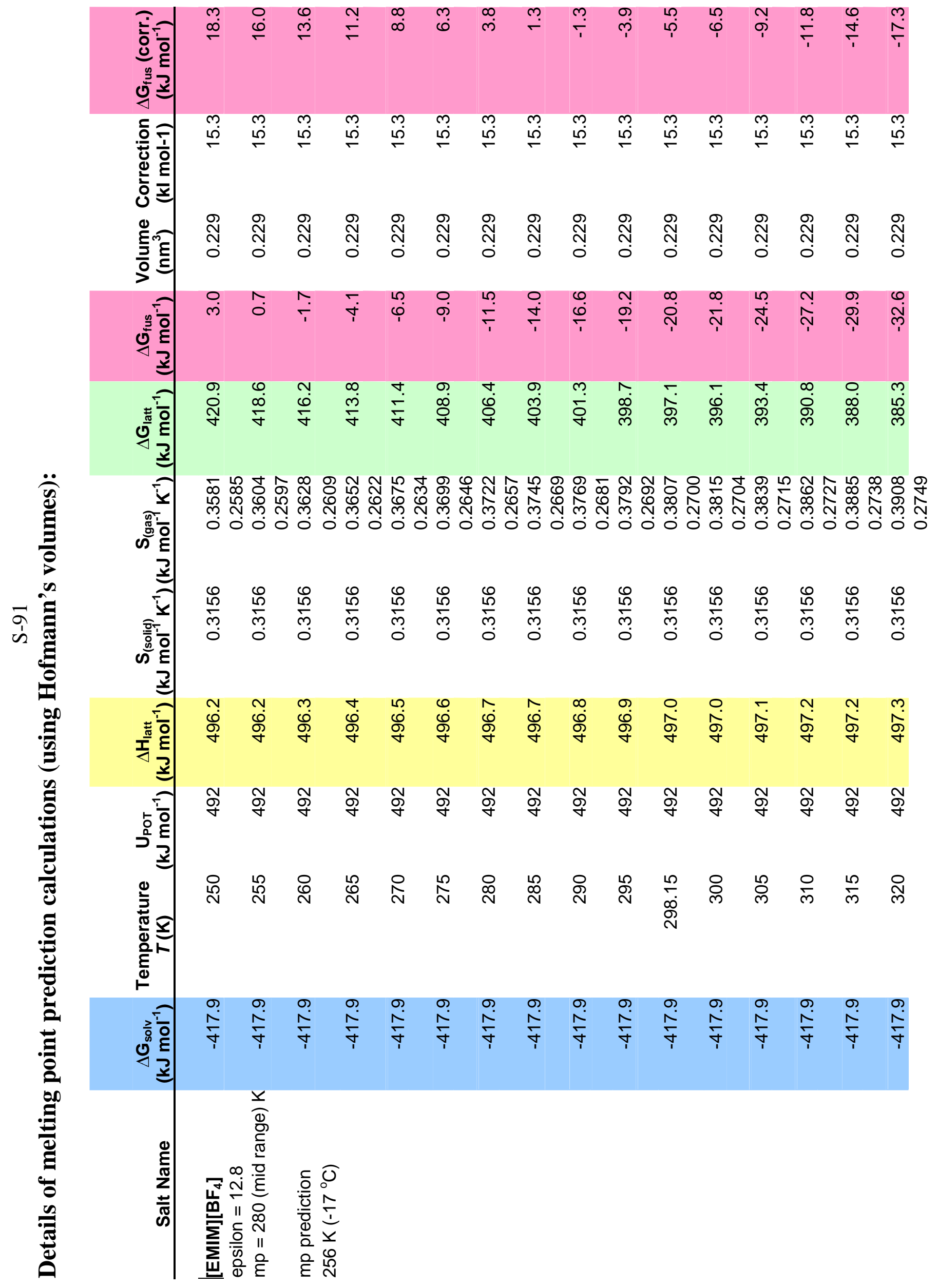




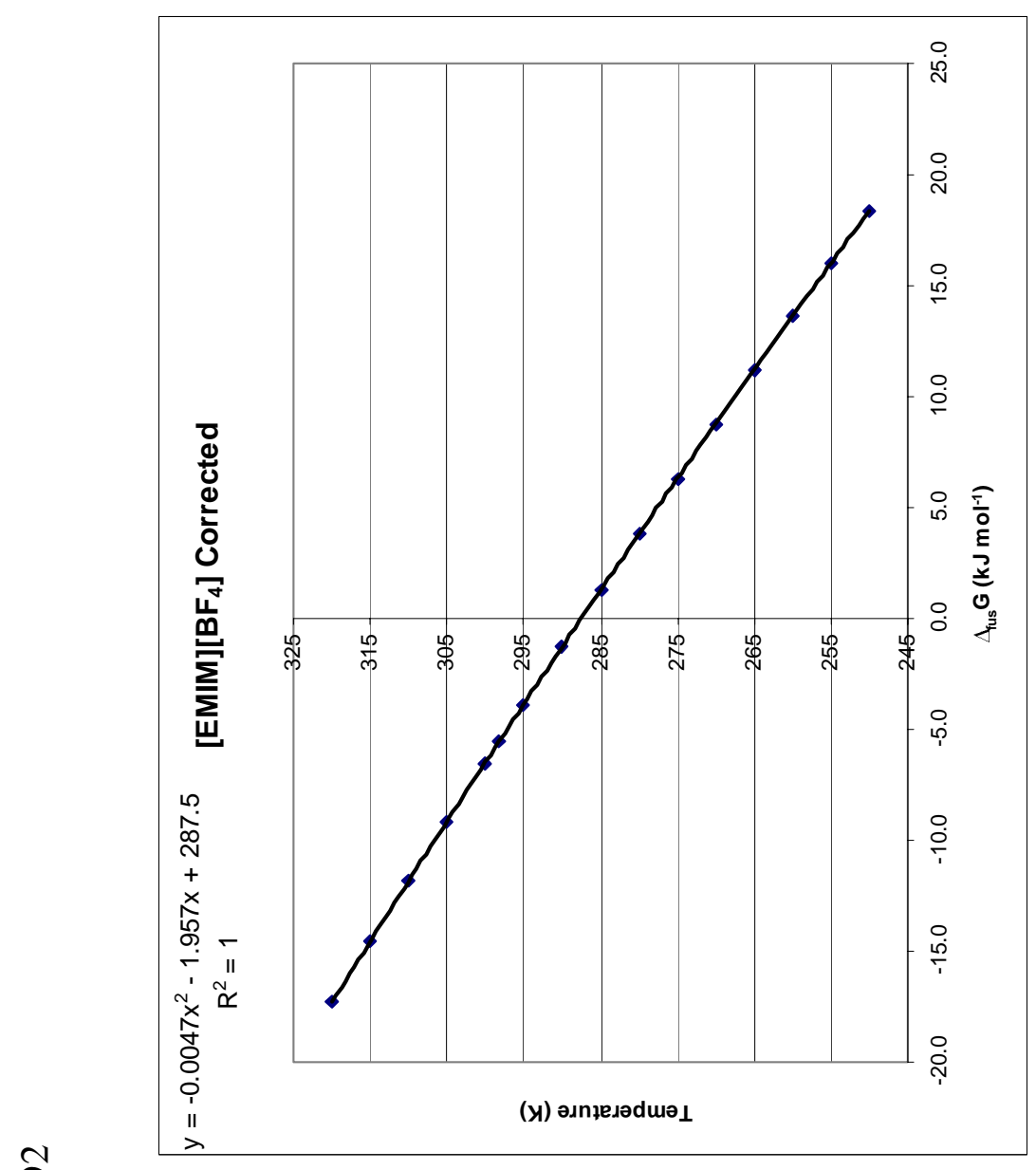

ô

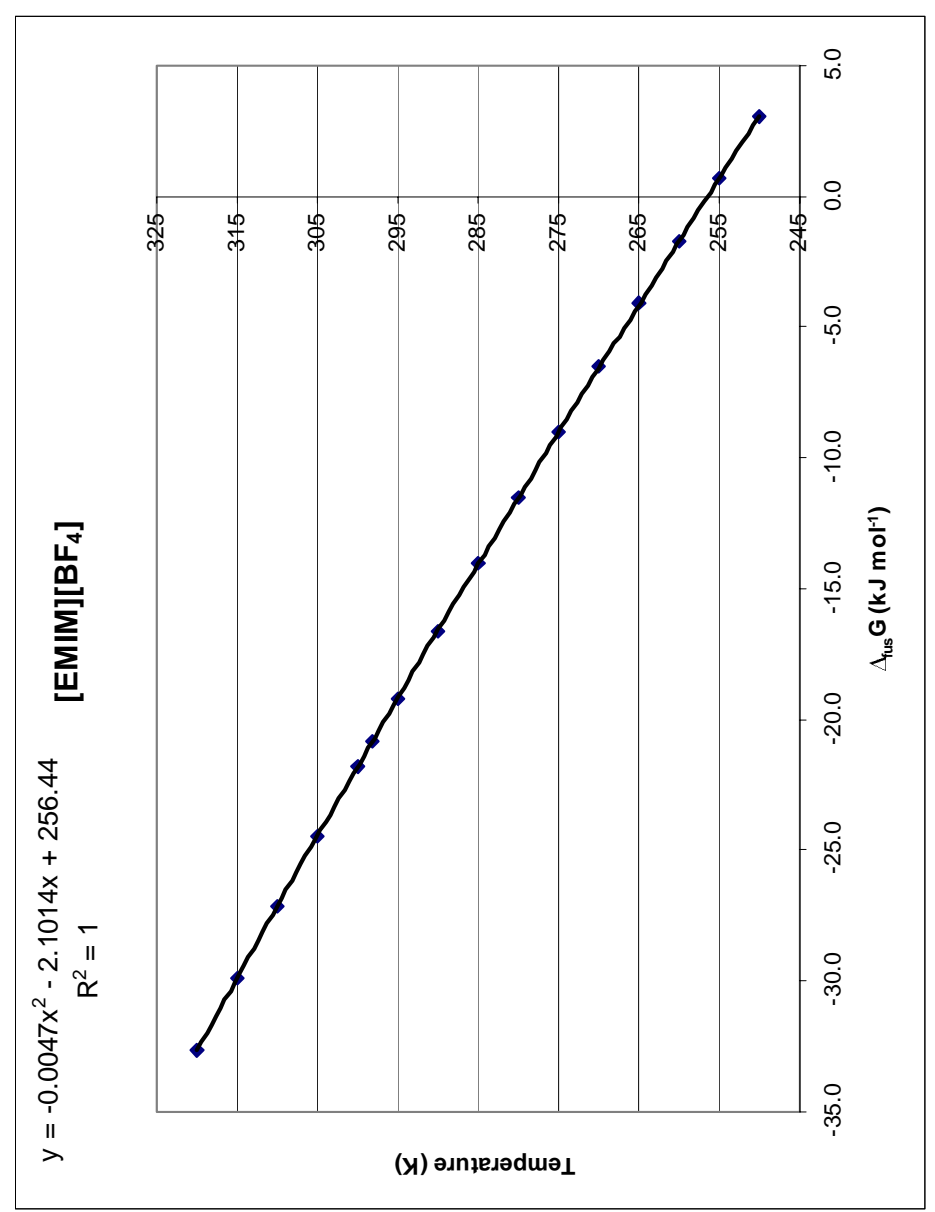




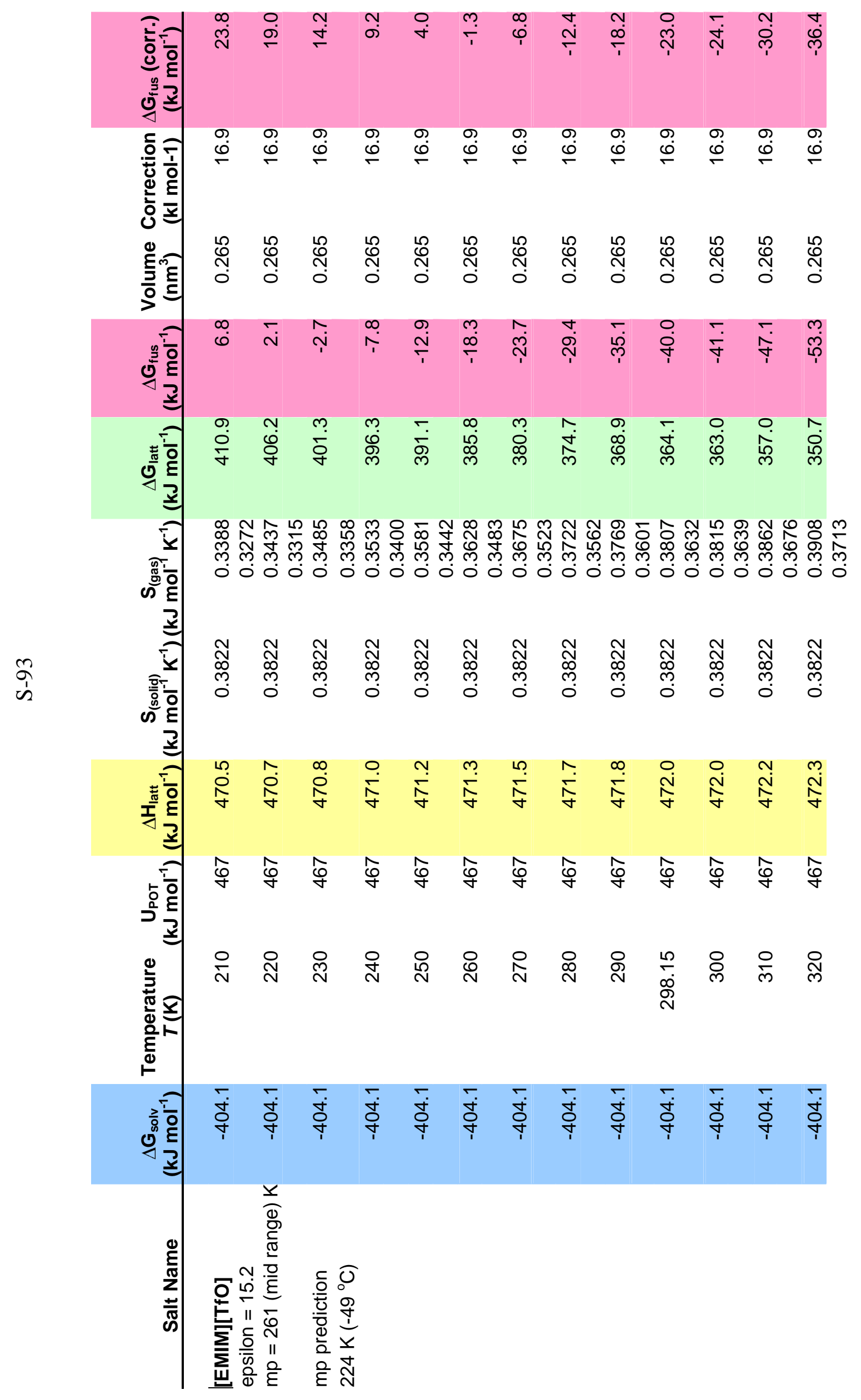




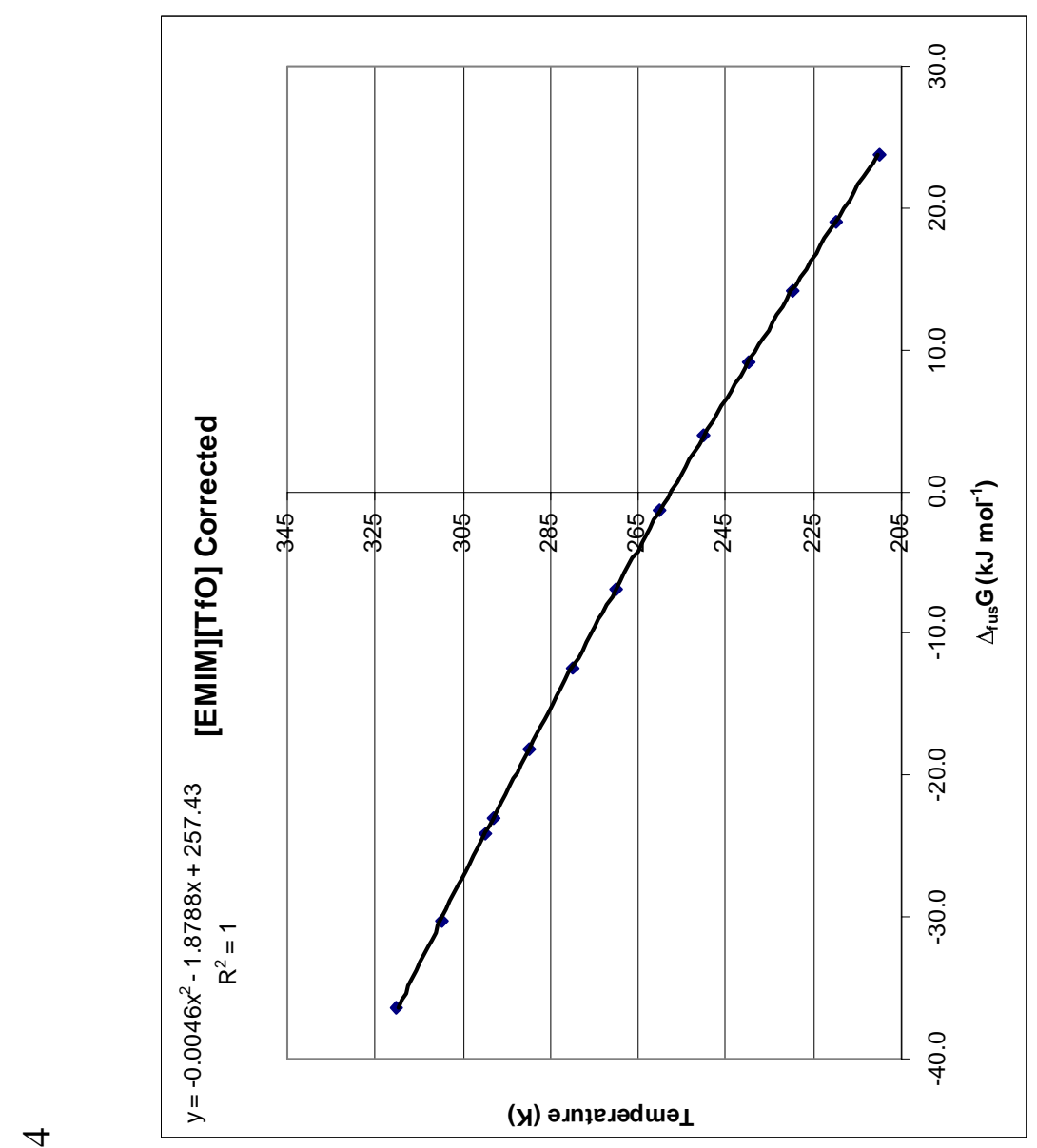

ลे

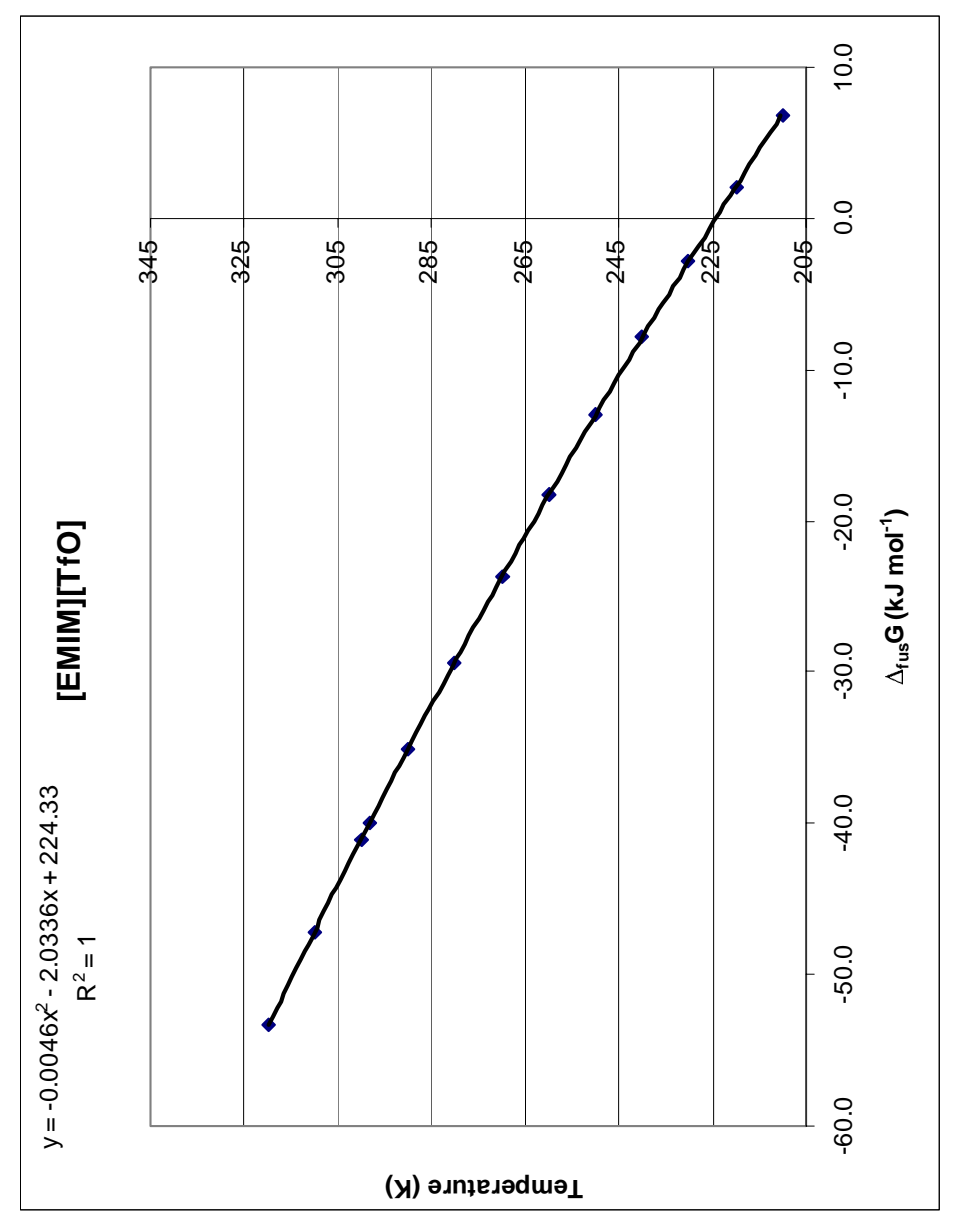




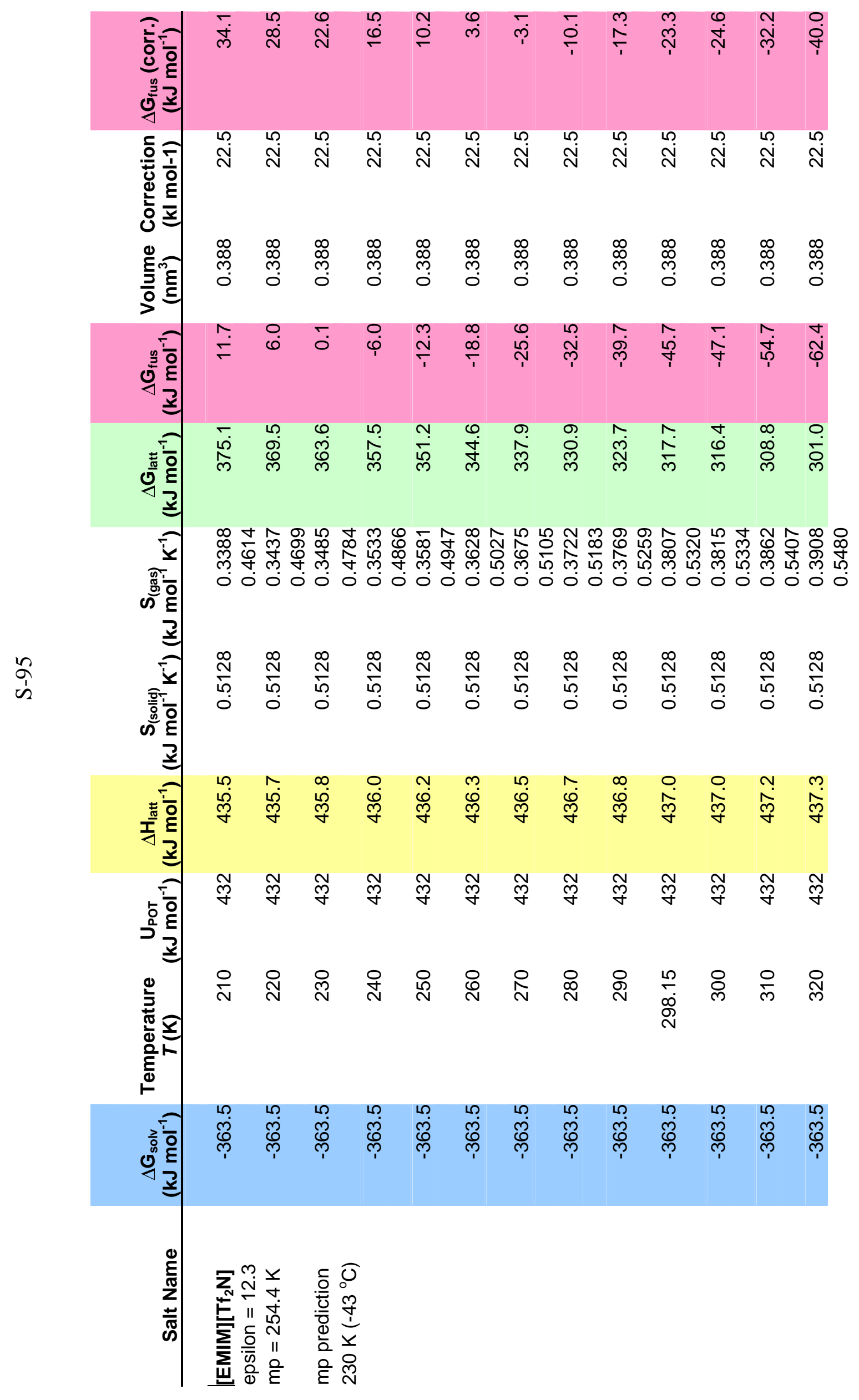




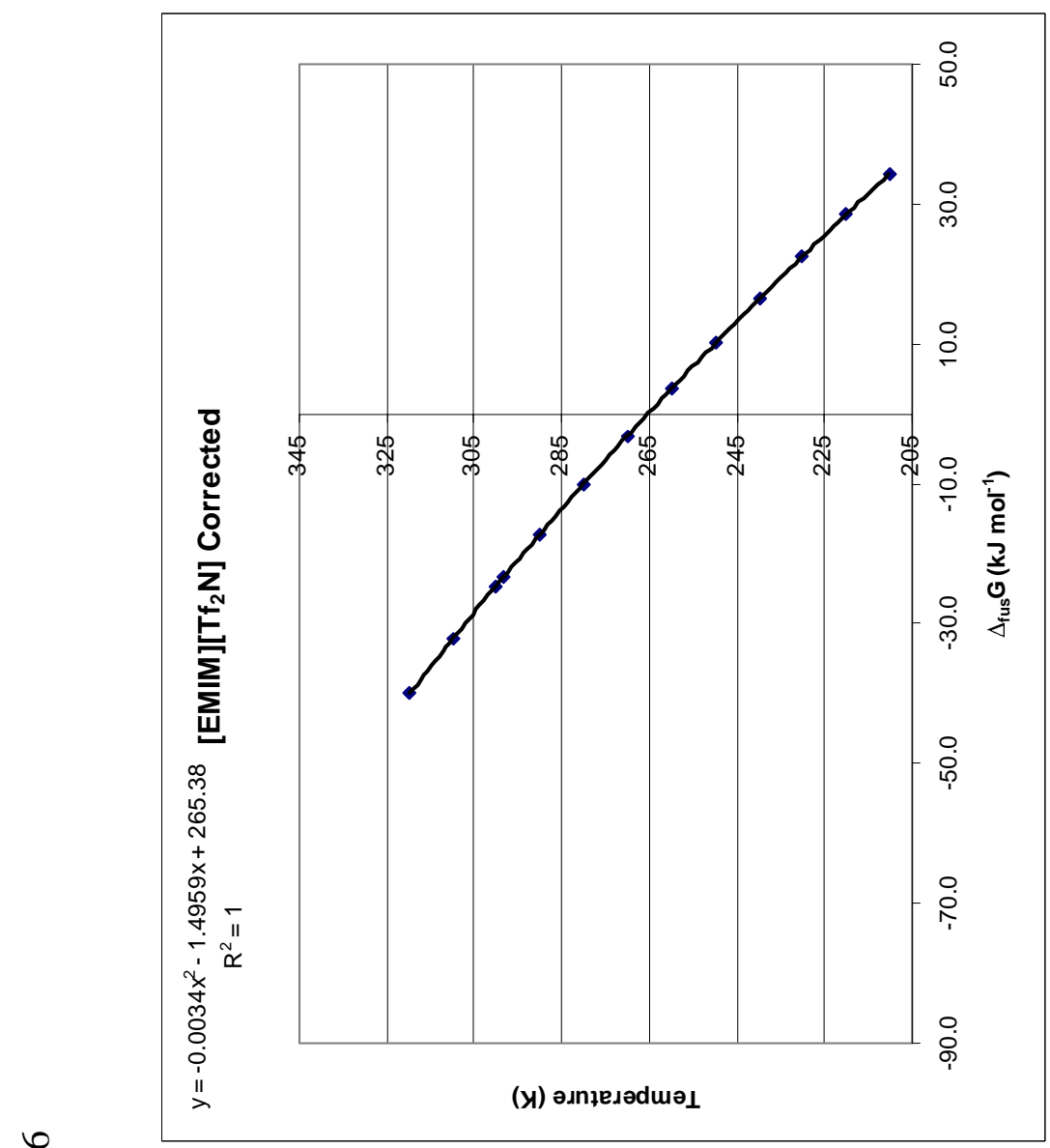

0
1े

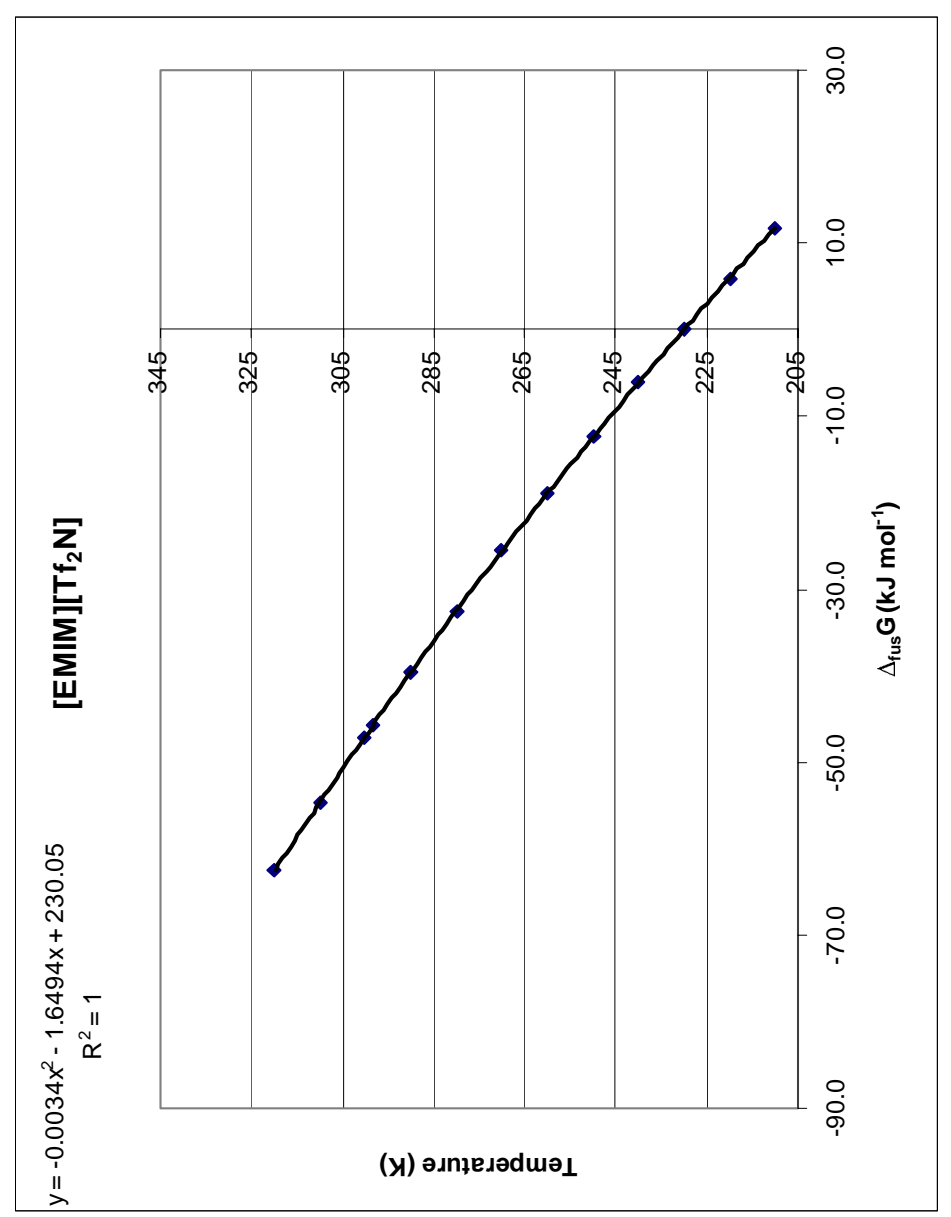




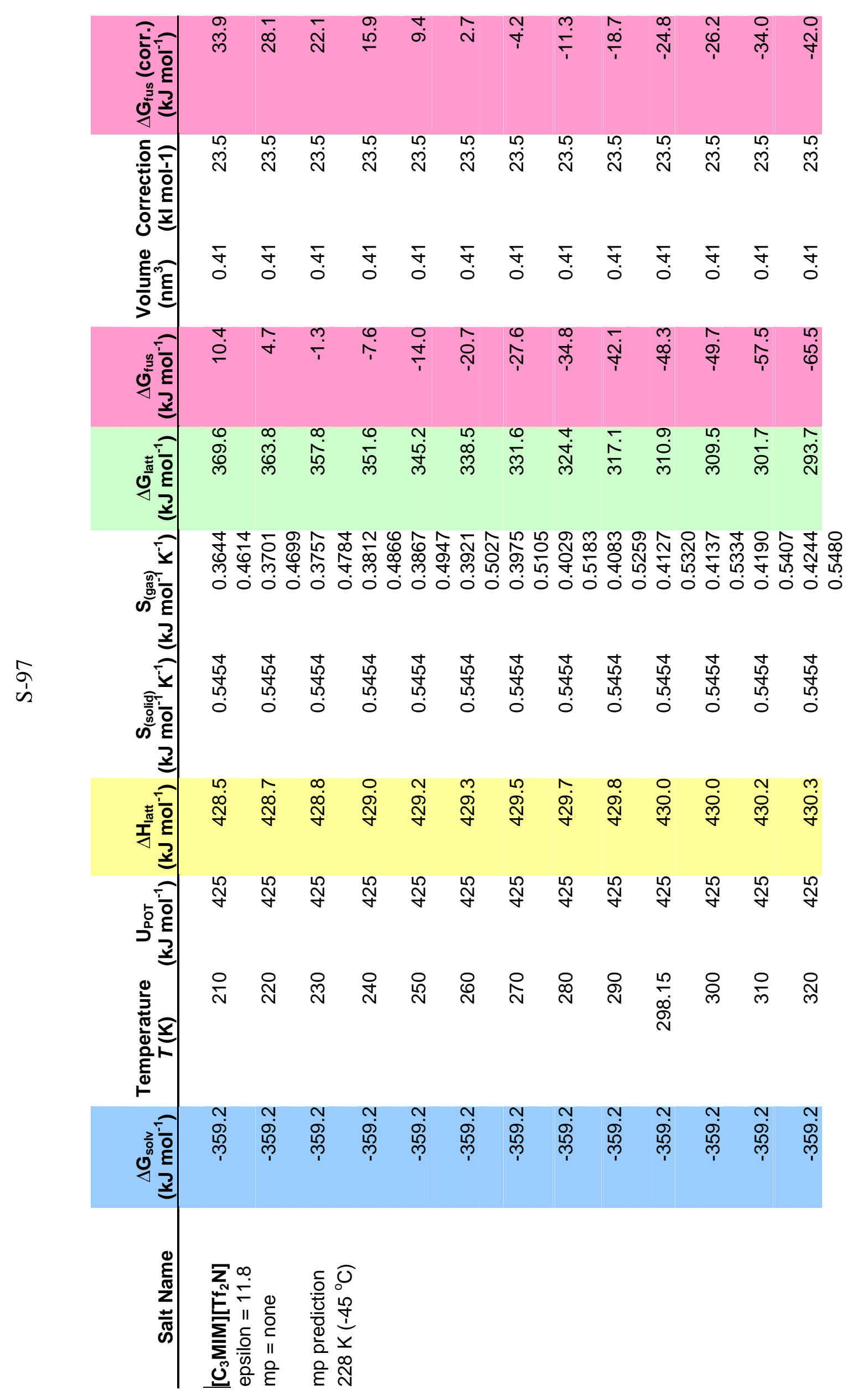




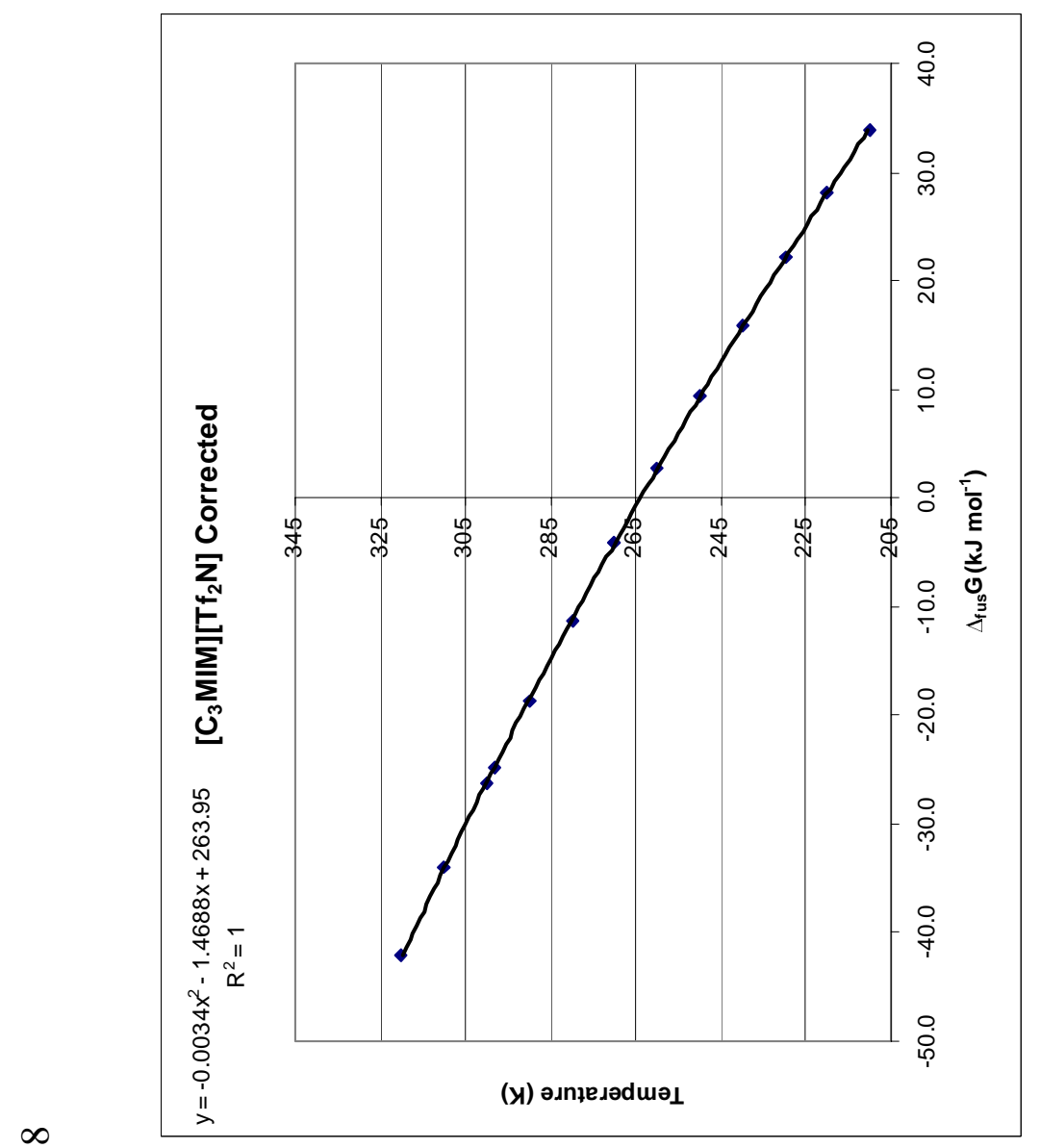

$\infty$
$a$
$i$

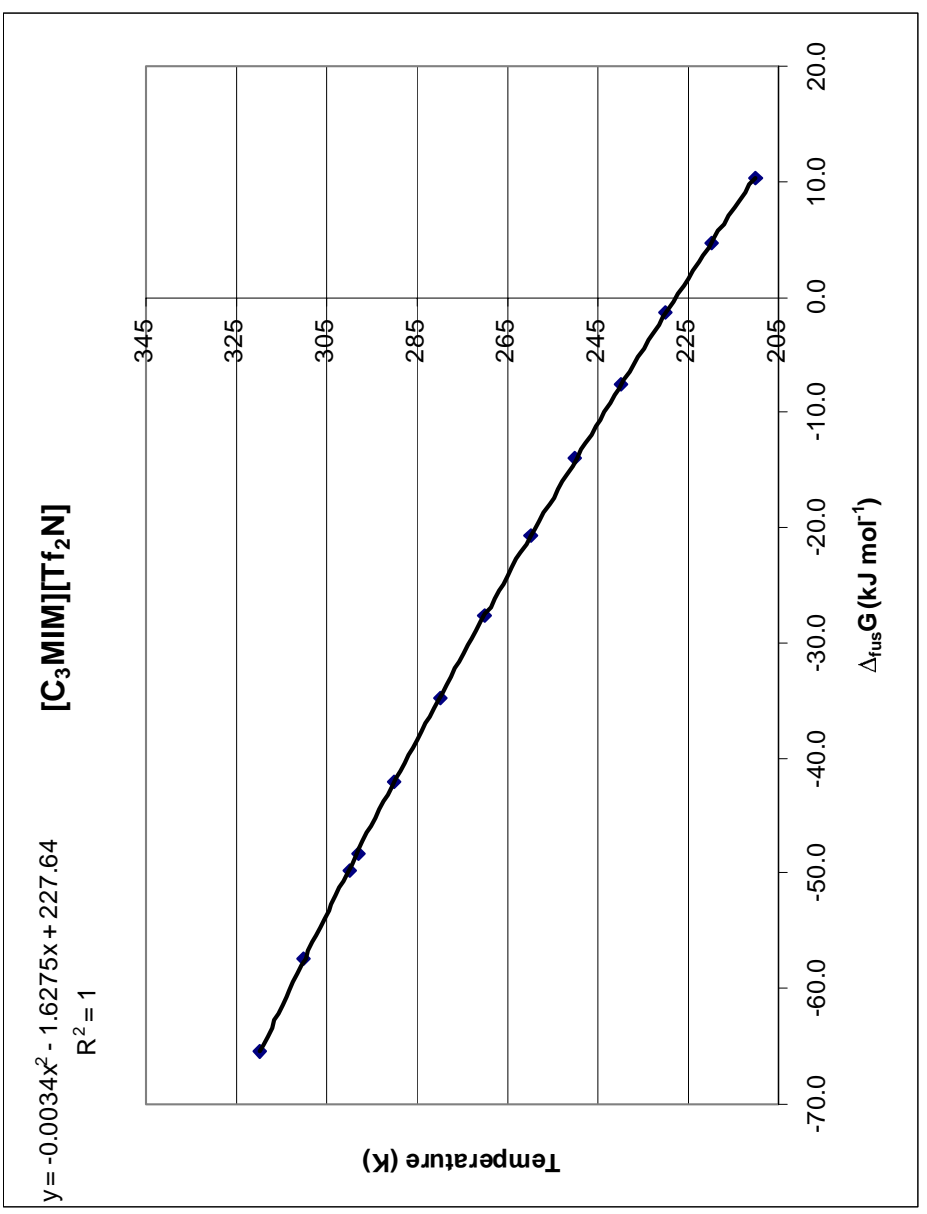




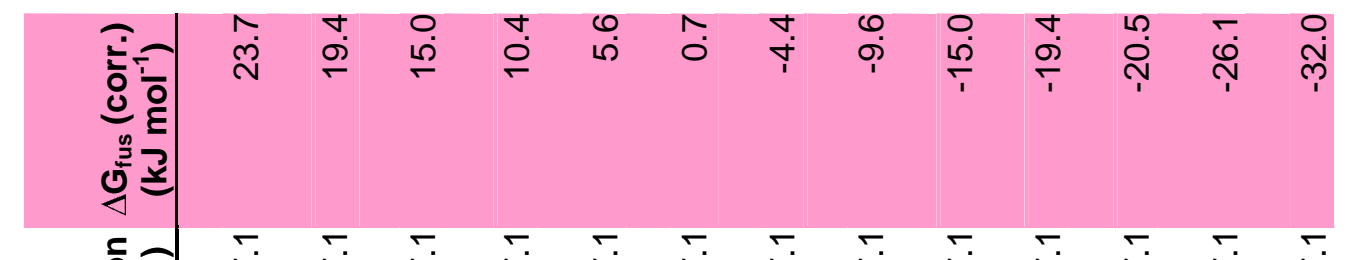

$$
\begin{aligned}
& \text { 호홀 }
\end{aligned}
$$

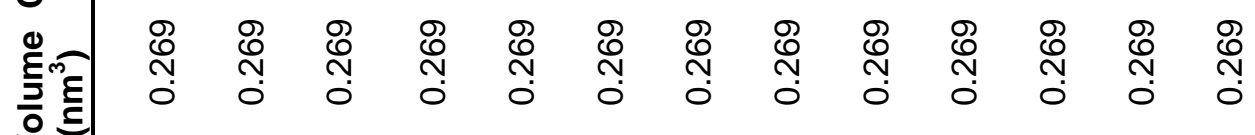

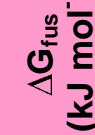

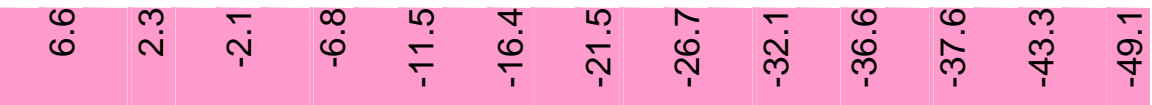

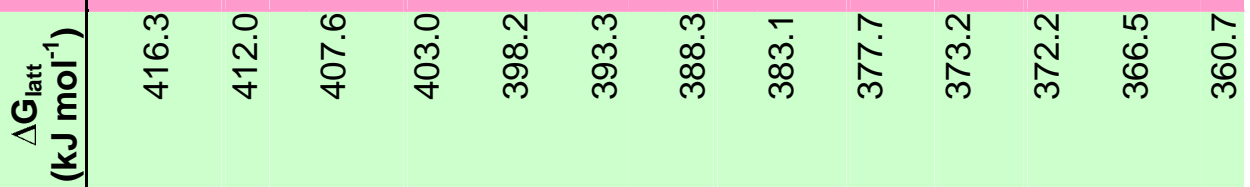

$$
\begin{aligned}
& \text { ป }
\end{aligned}
$$

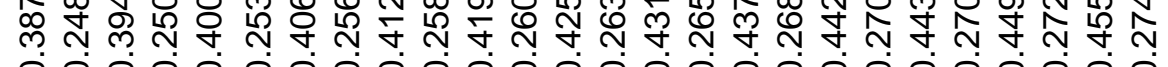

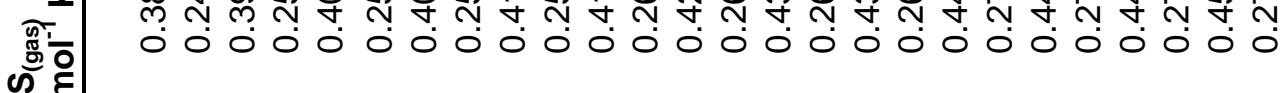

$$
\begin{aligned}
& \text { ? } \\
& \text { के }
\end{aligned}
$$

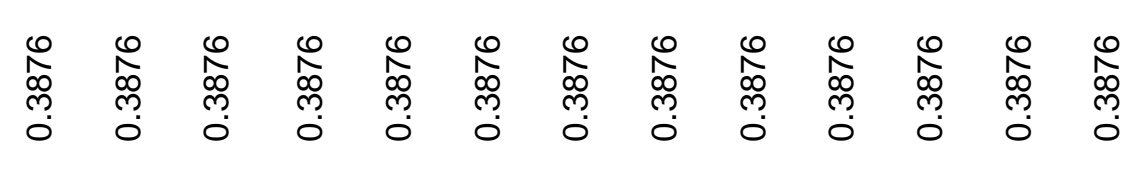

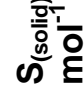

$$
\begin{aligned}
& \text { 항 }
\end{aligned}
$$

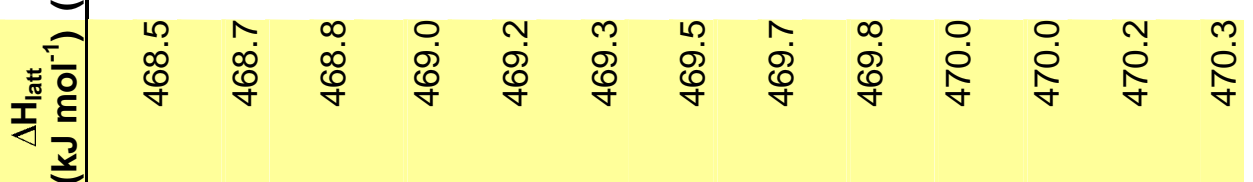

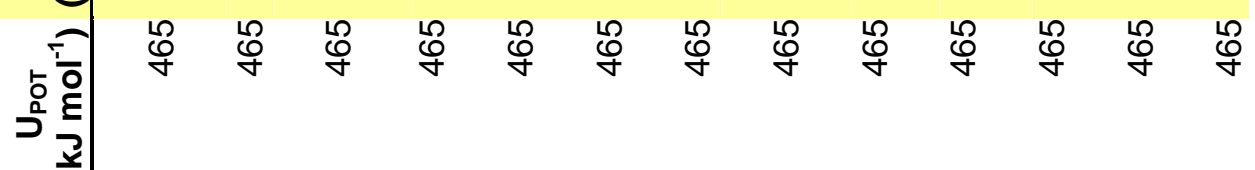

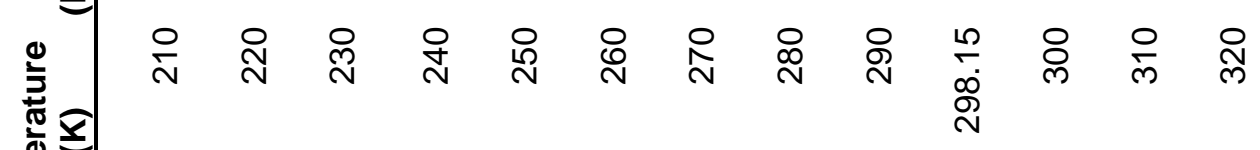

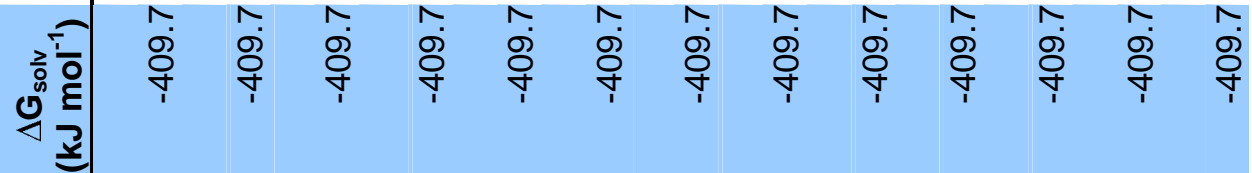

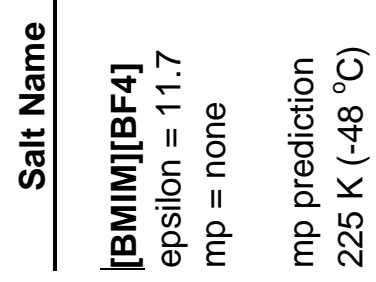



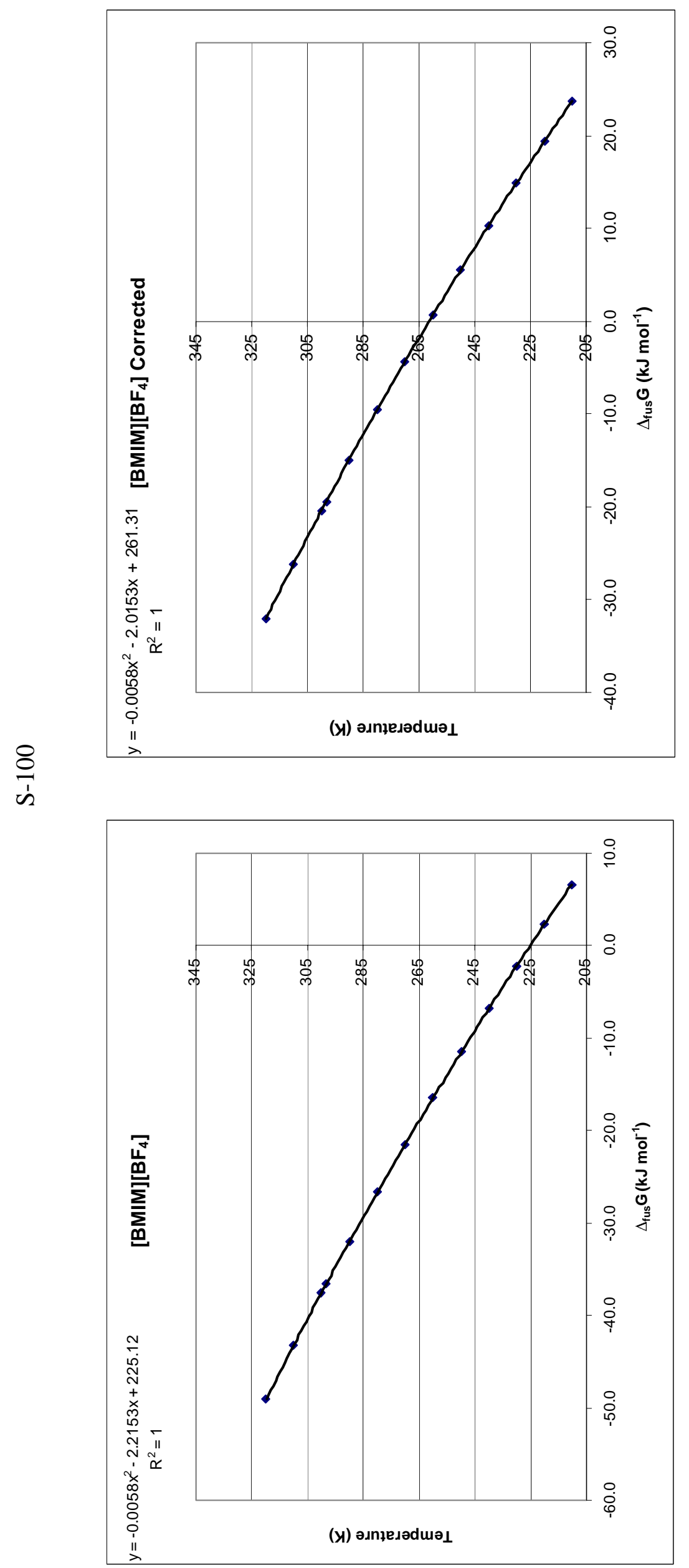


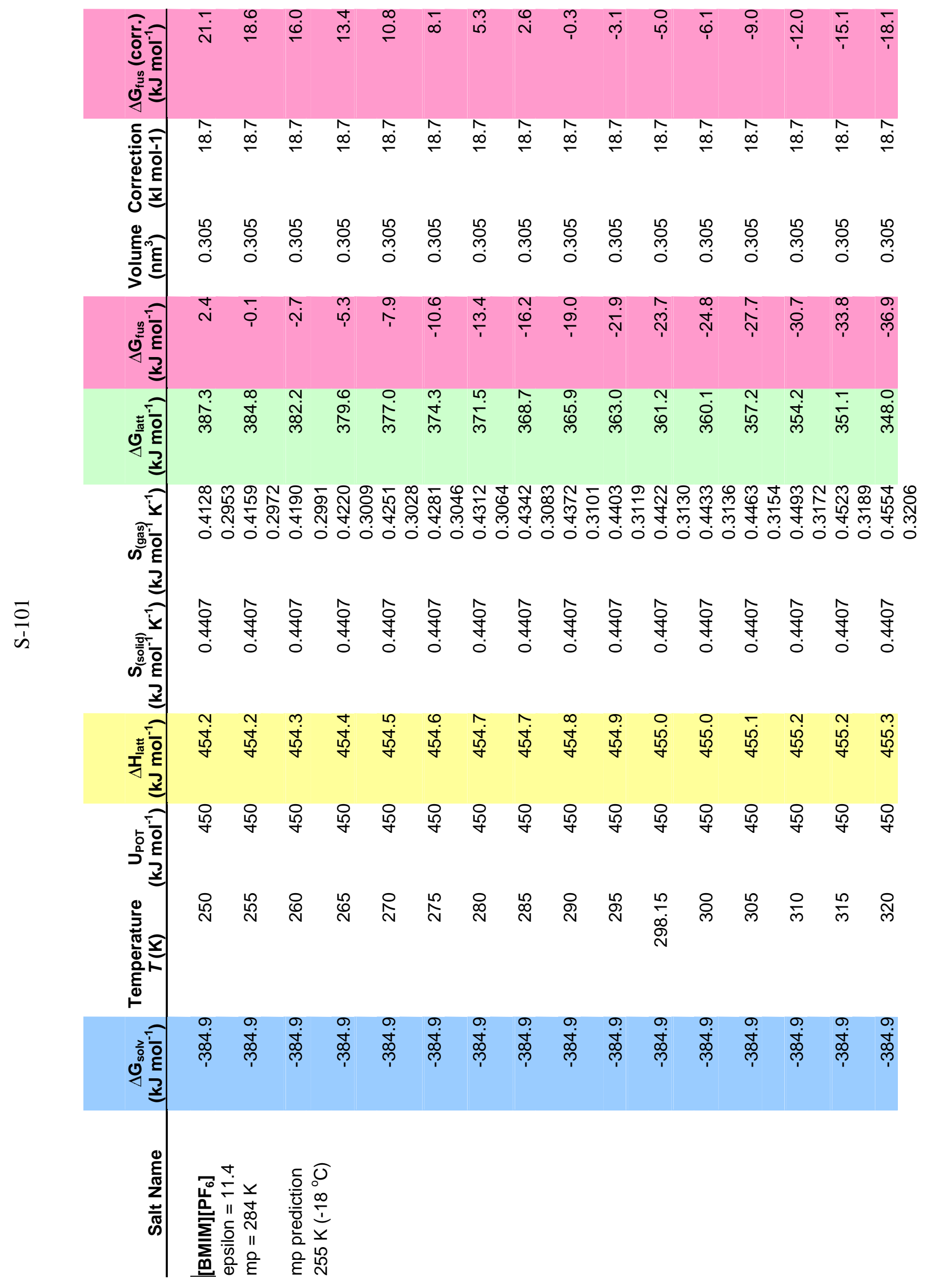



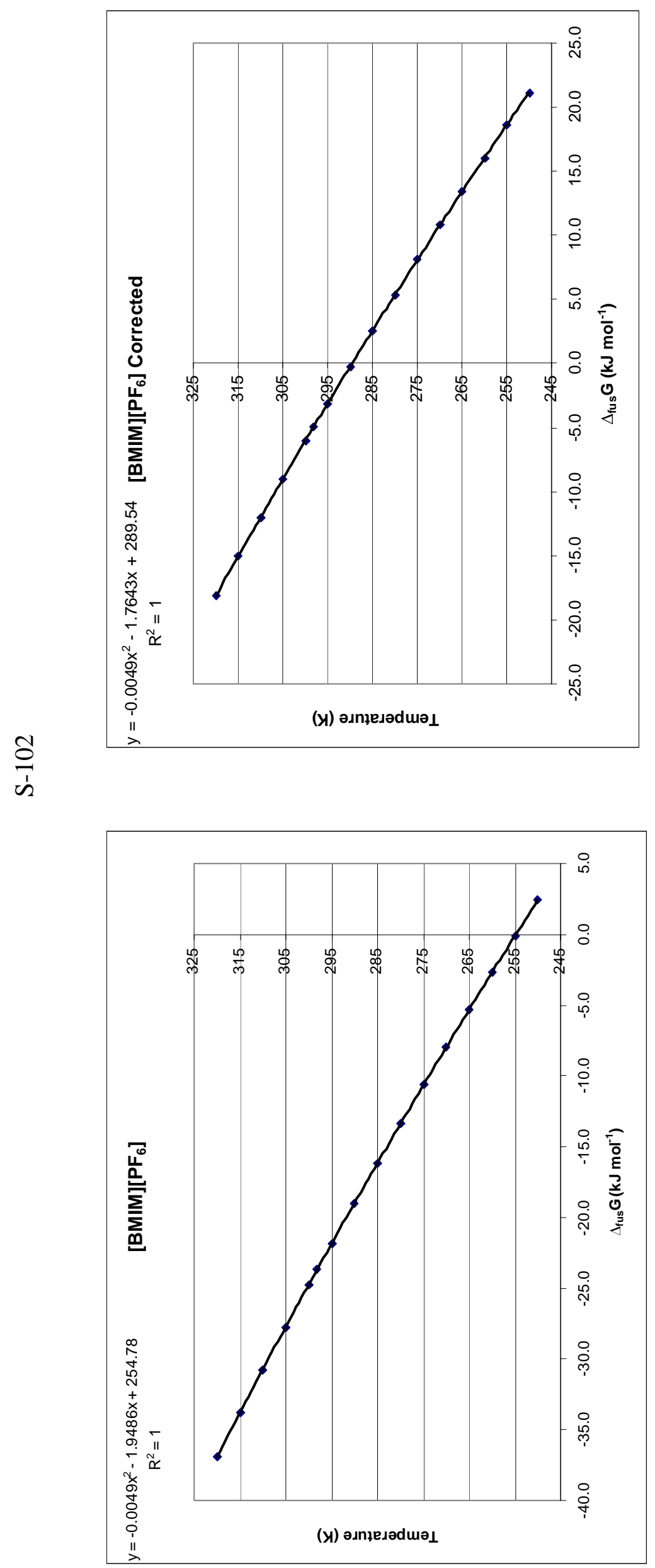


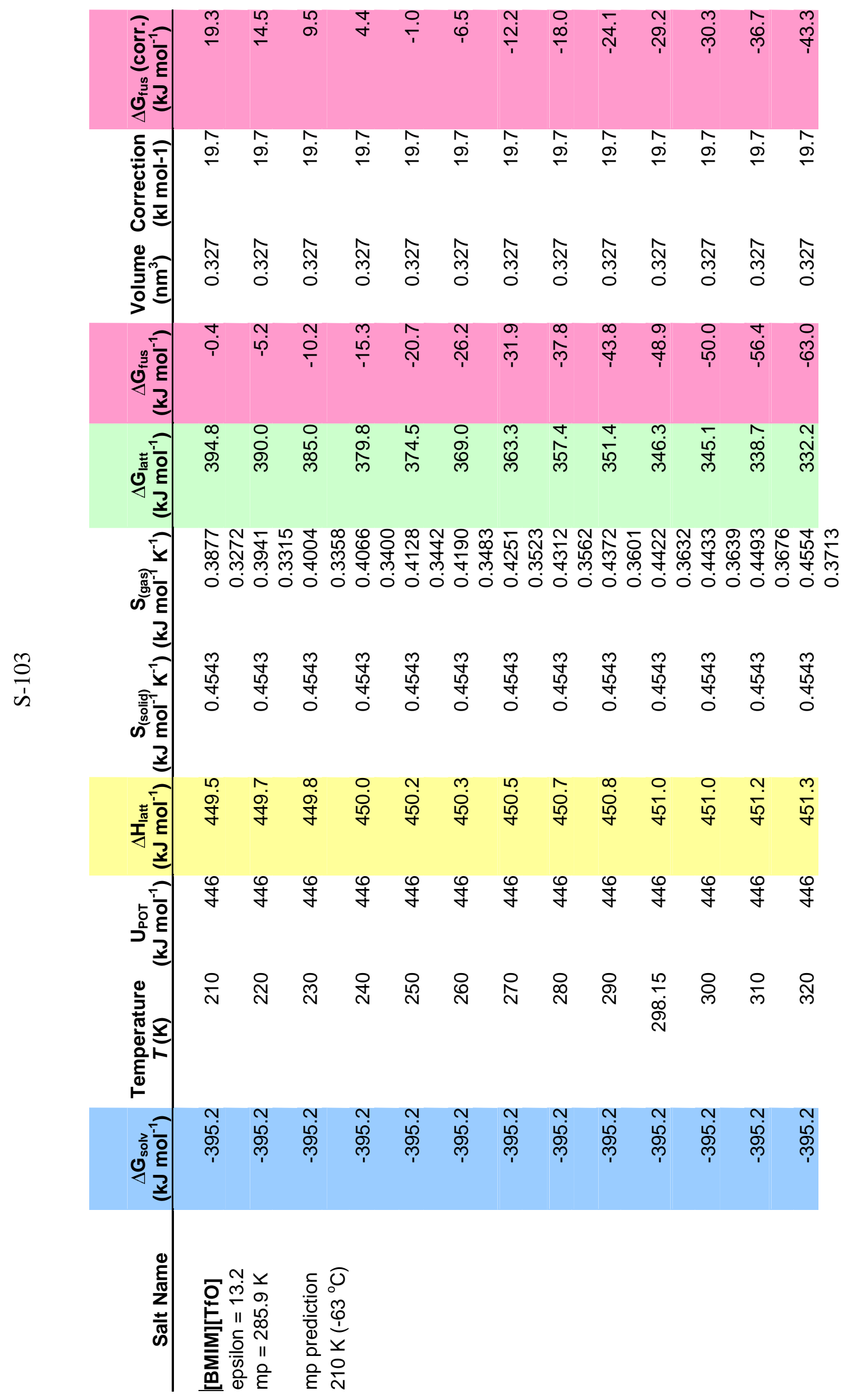



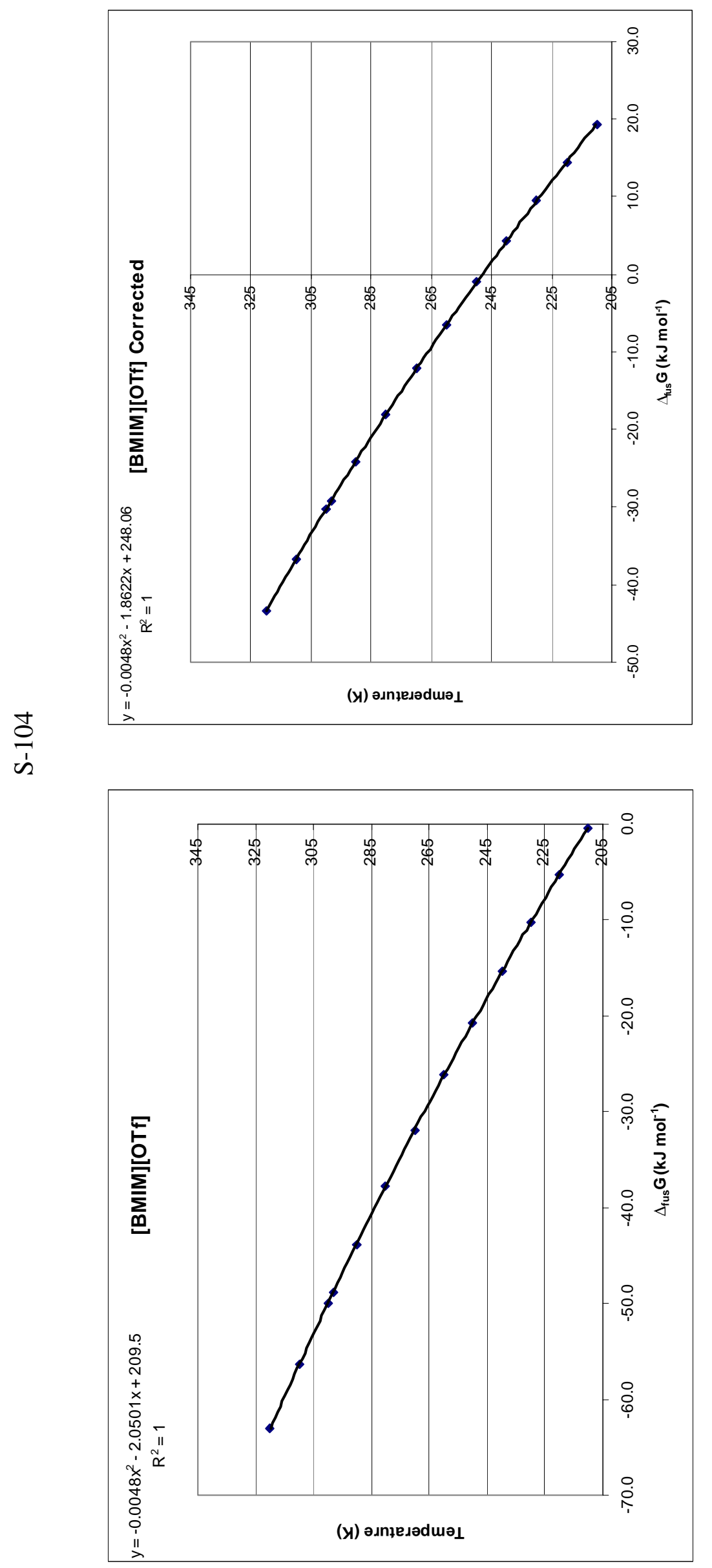


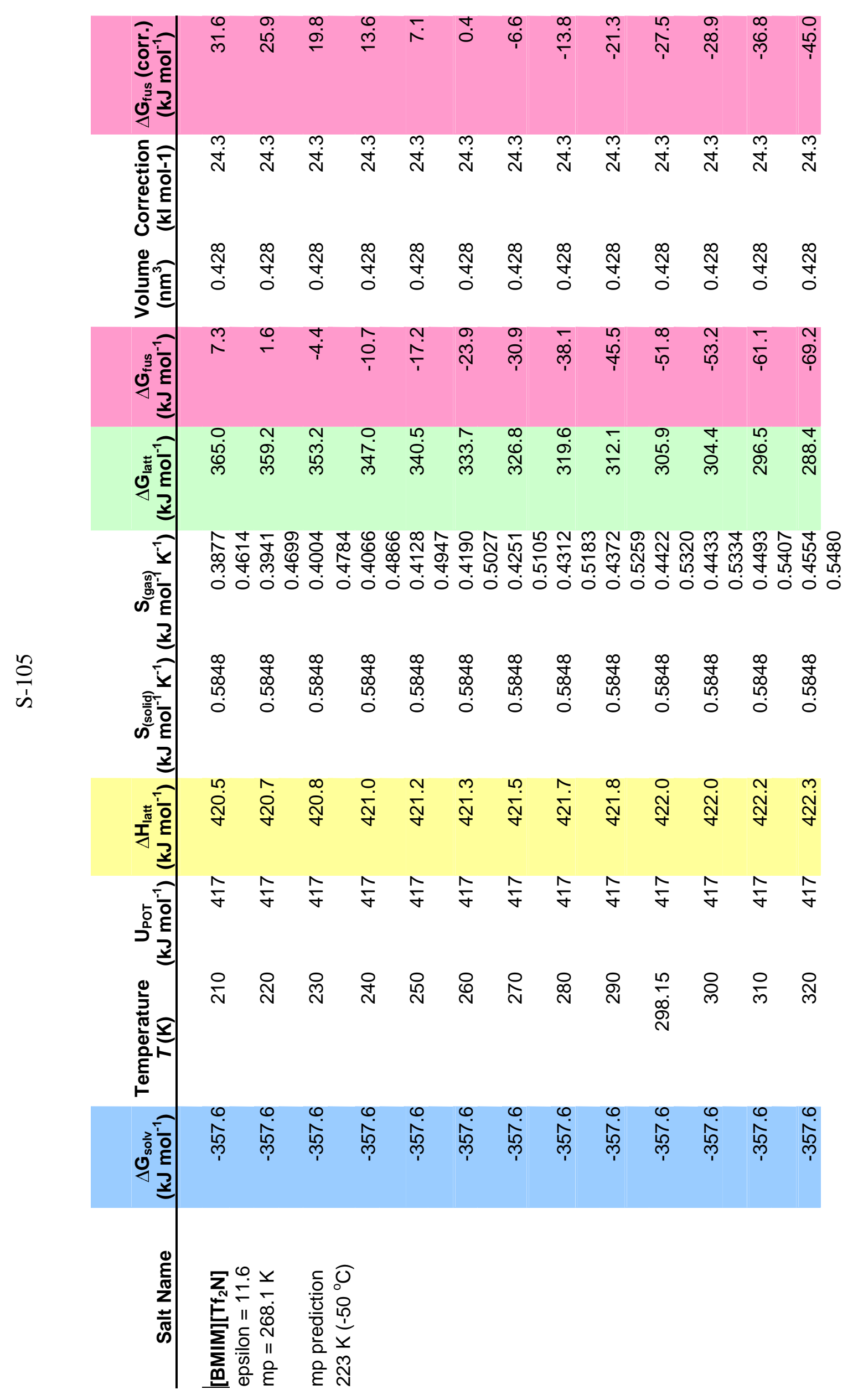



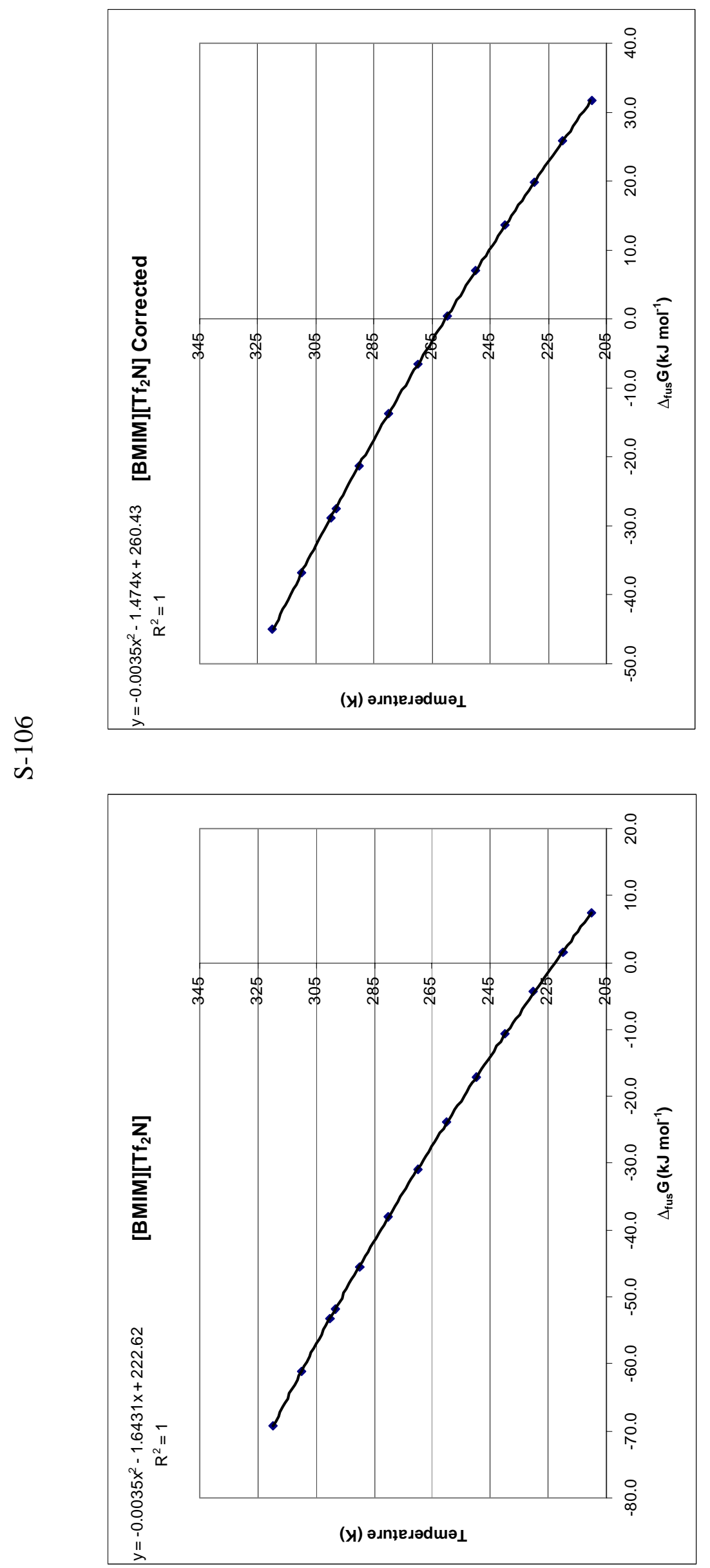


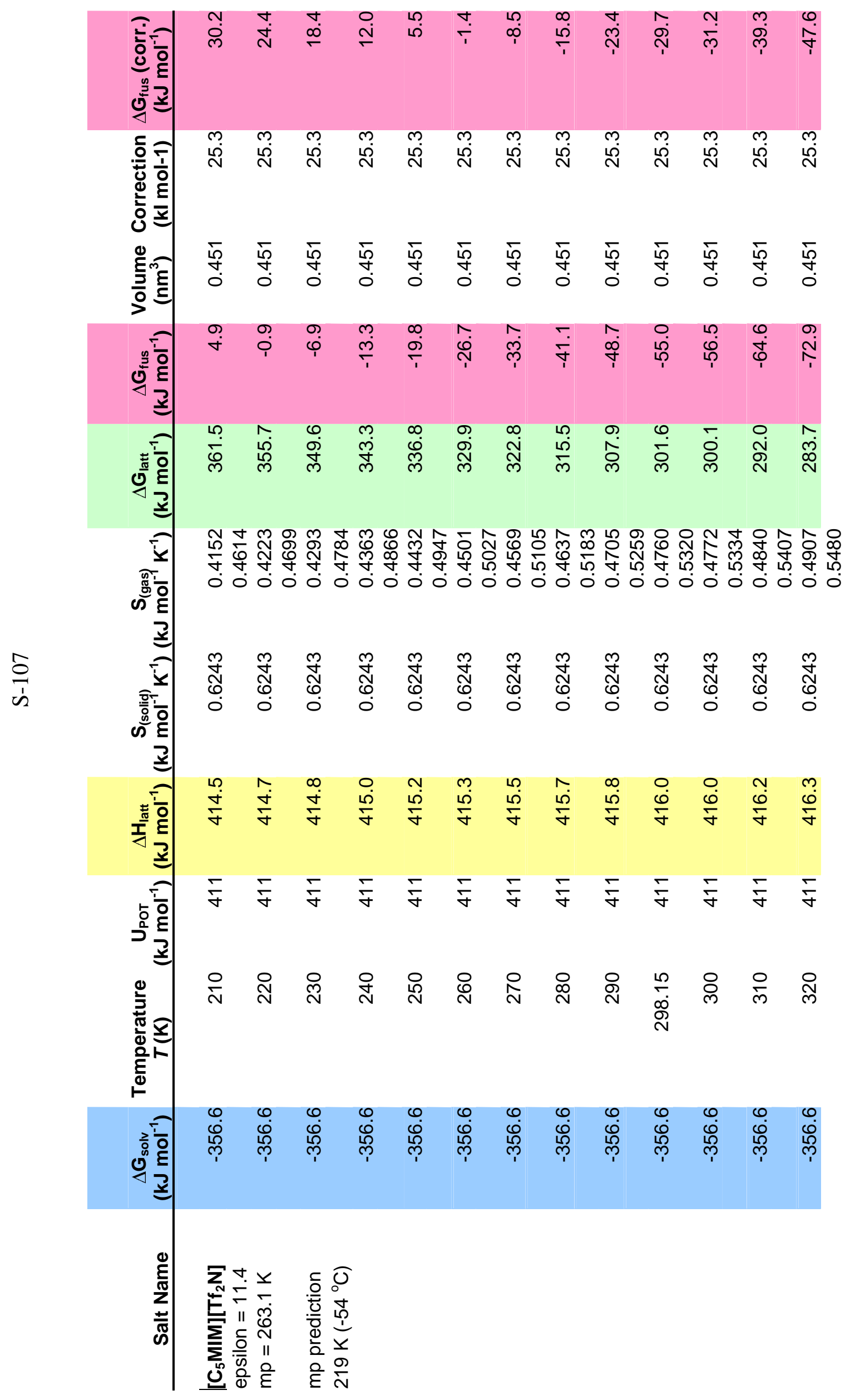




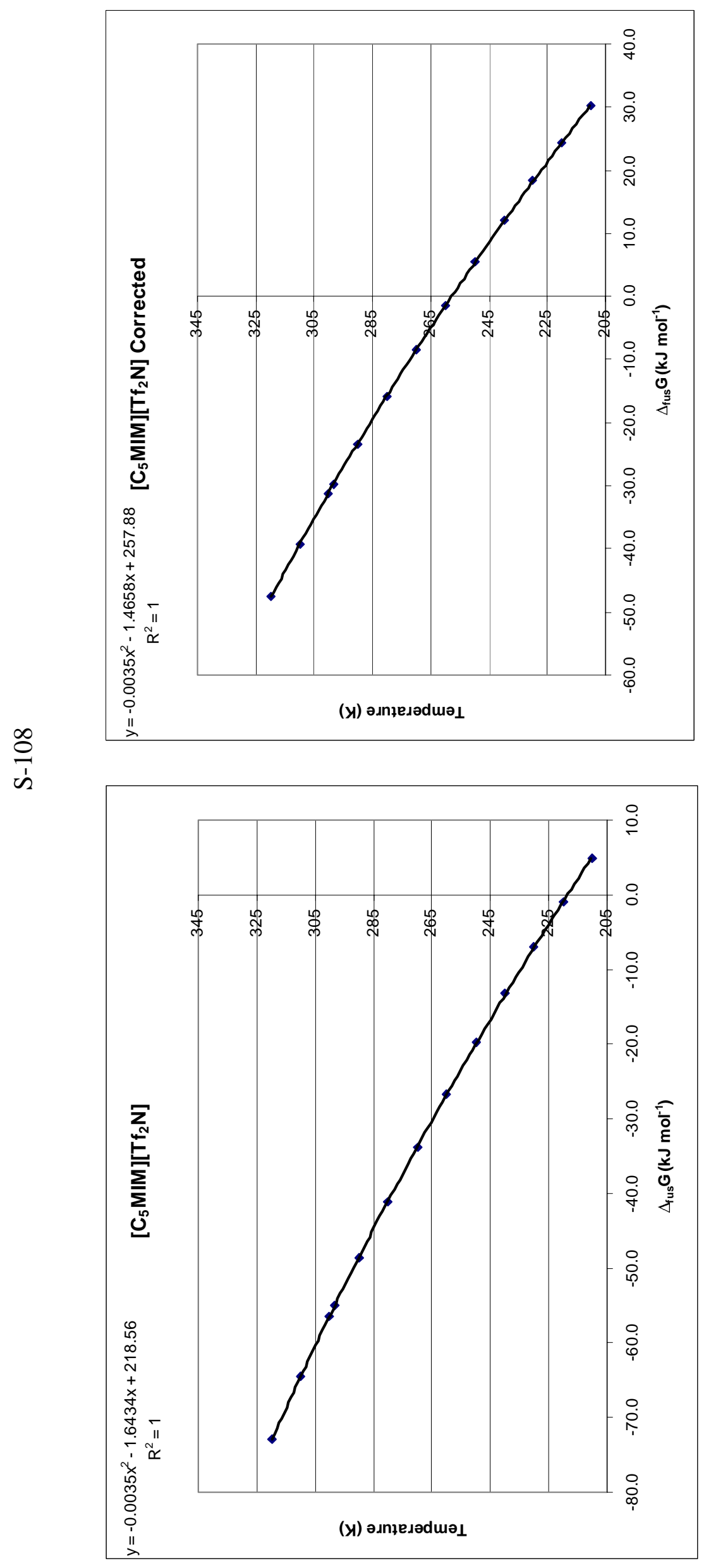




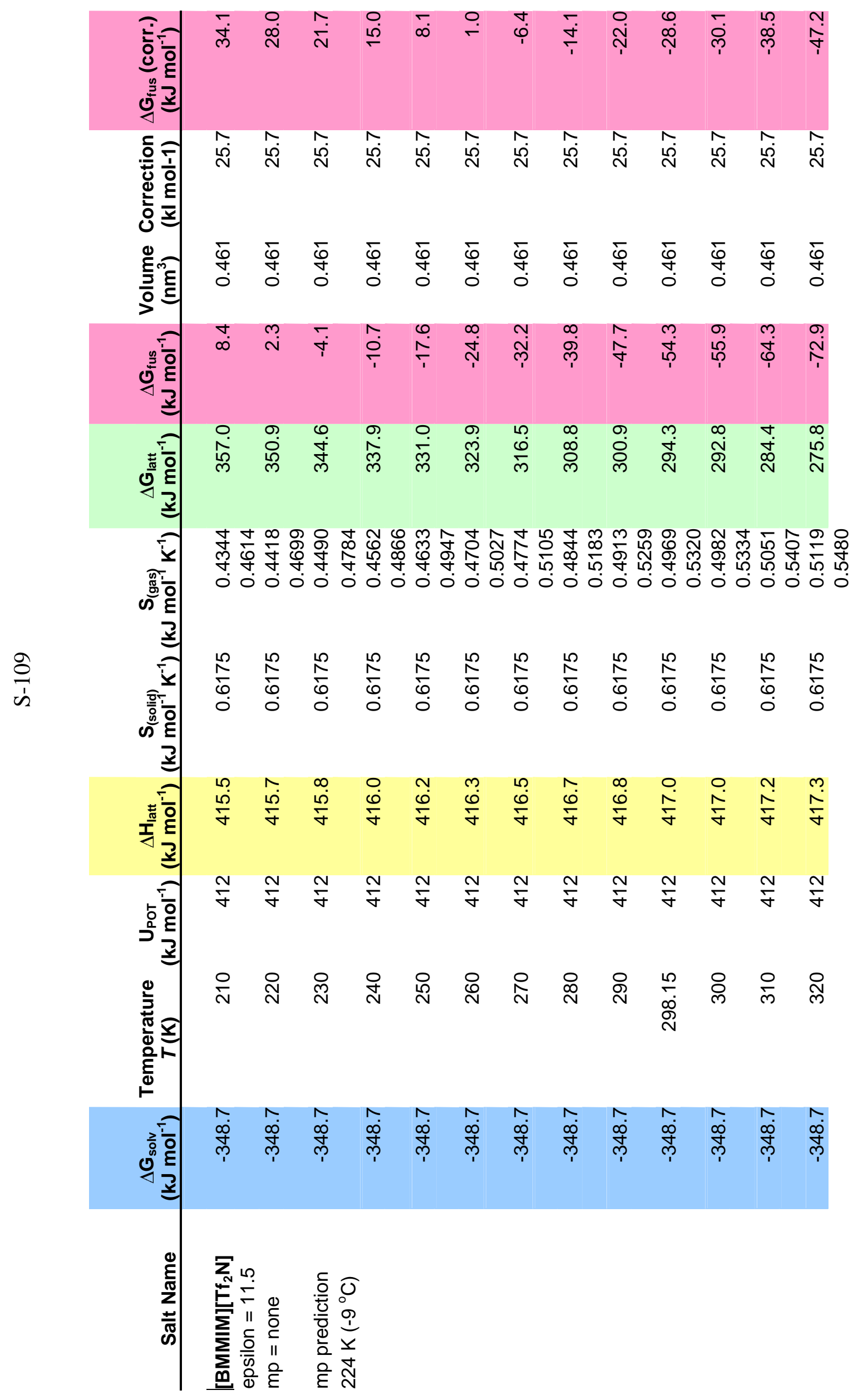



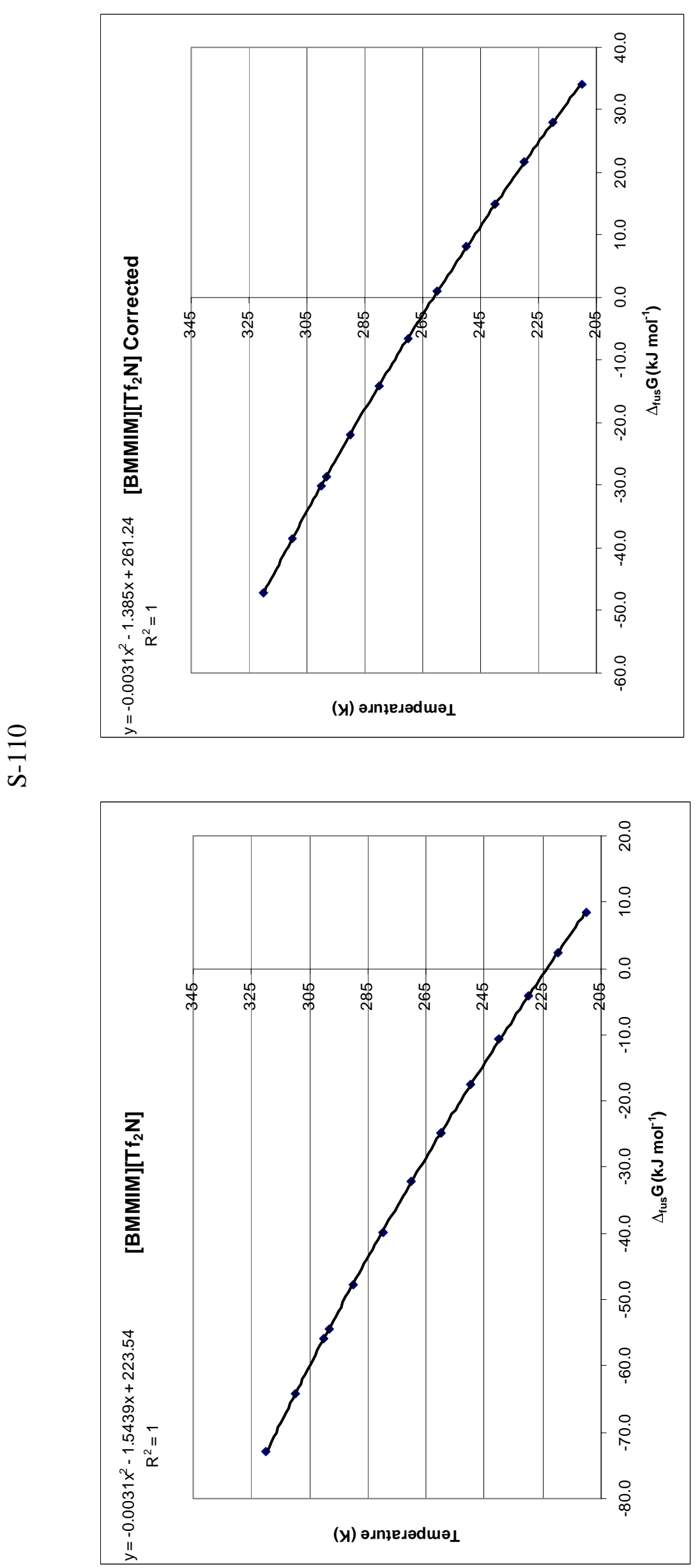


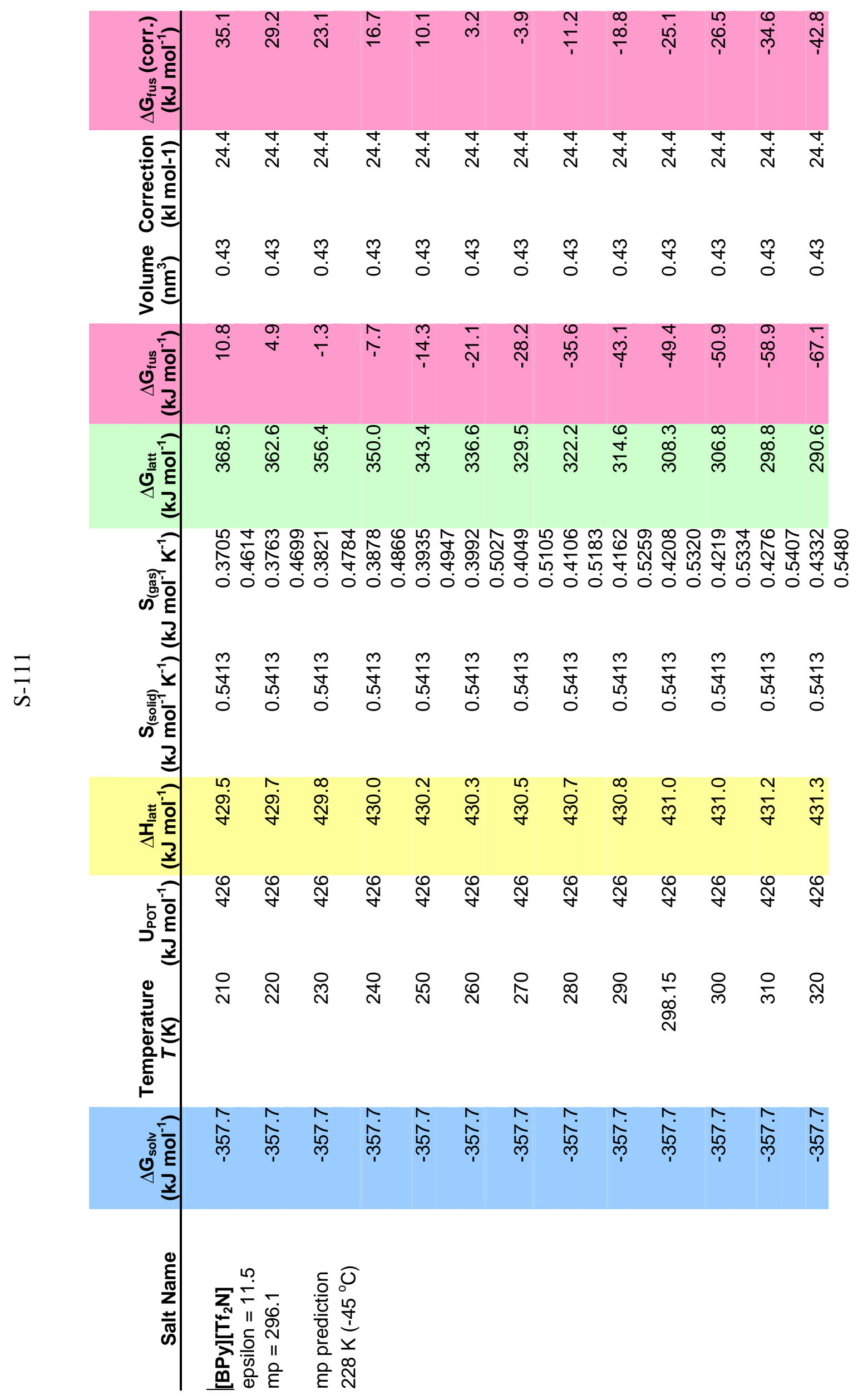



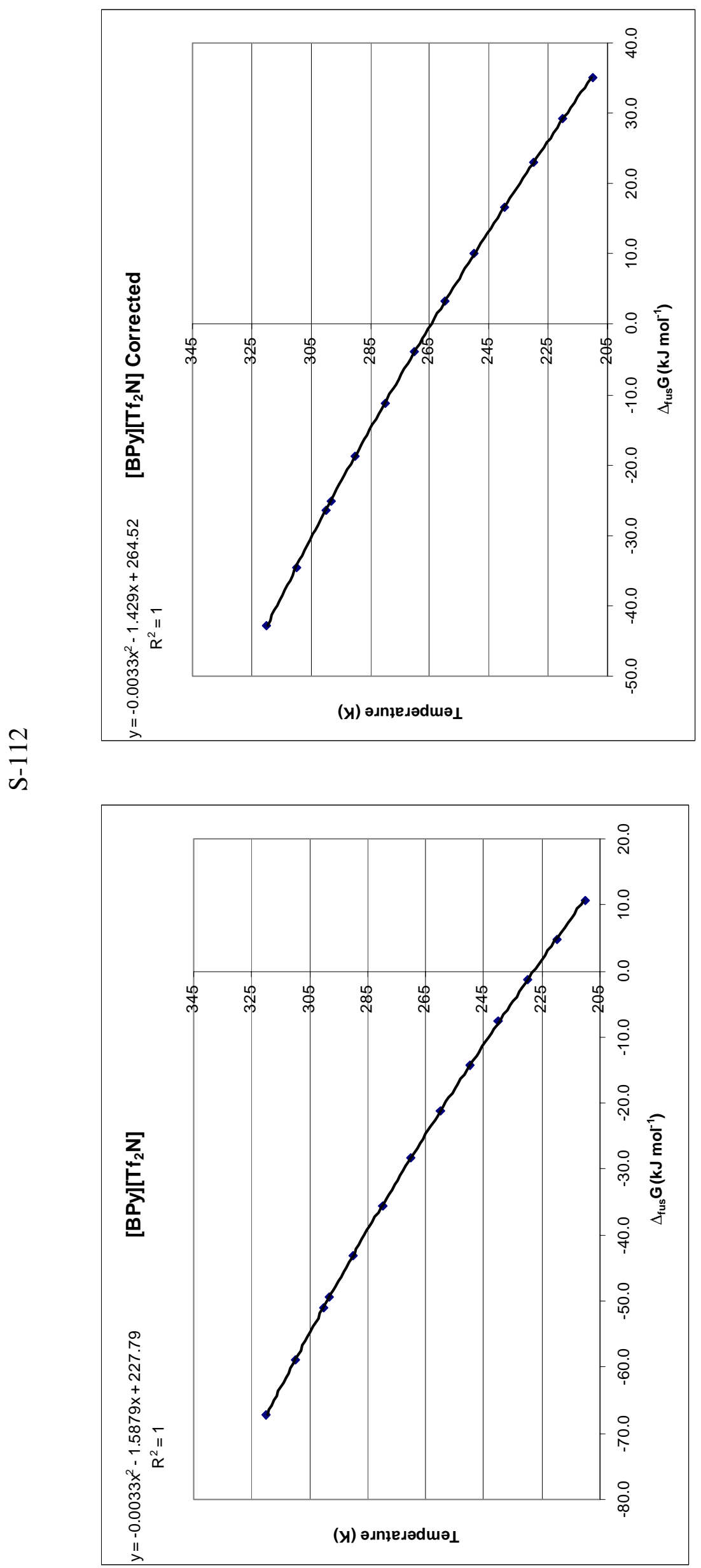


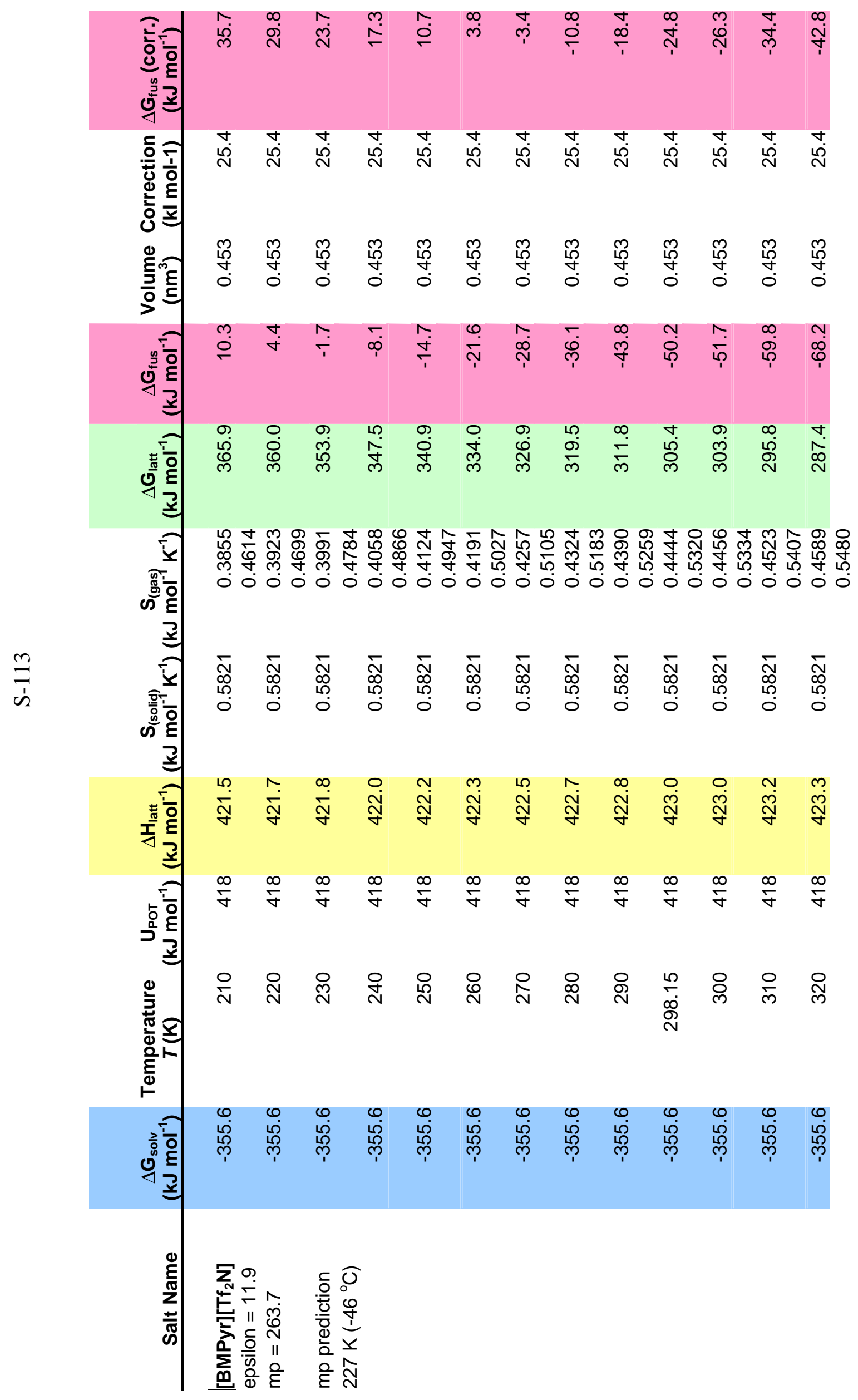




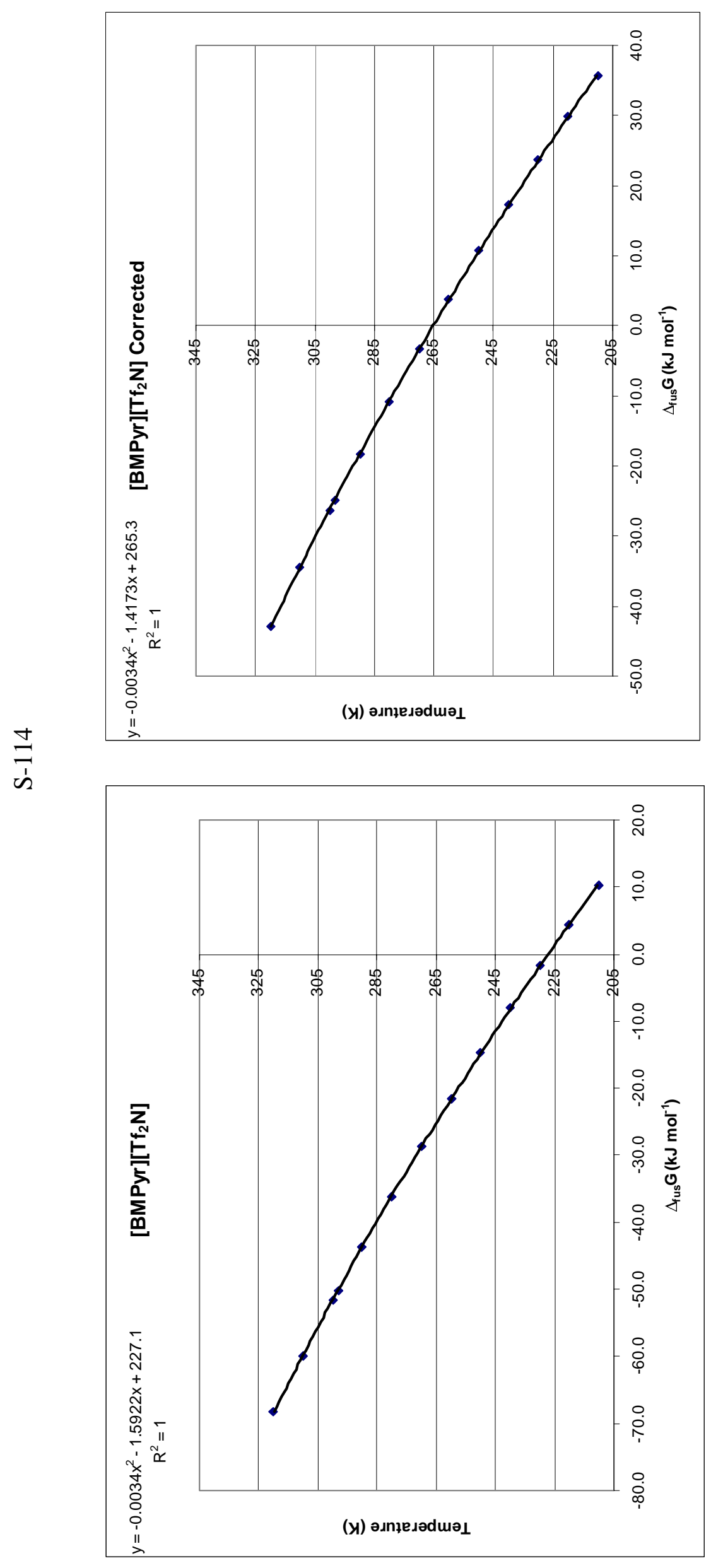




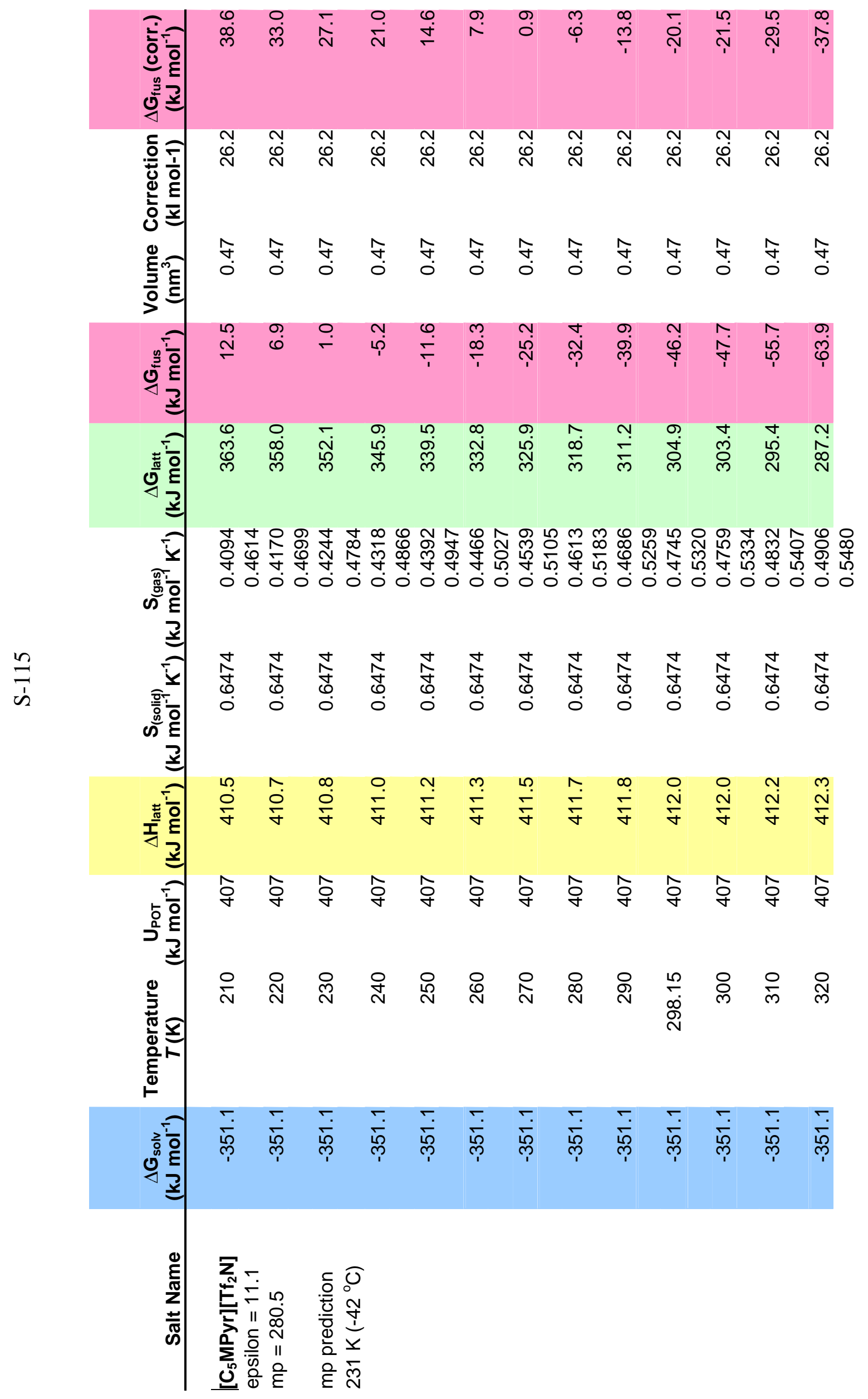



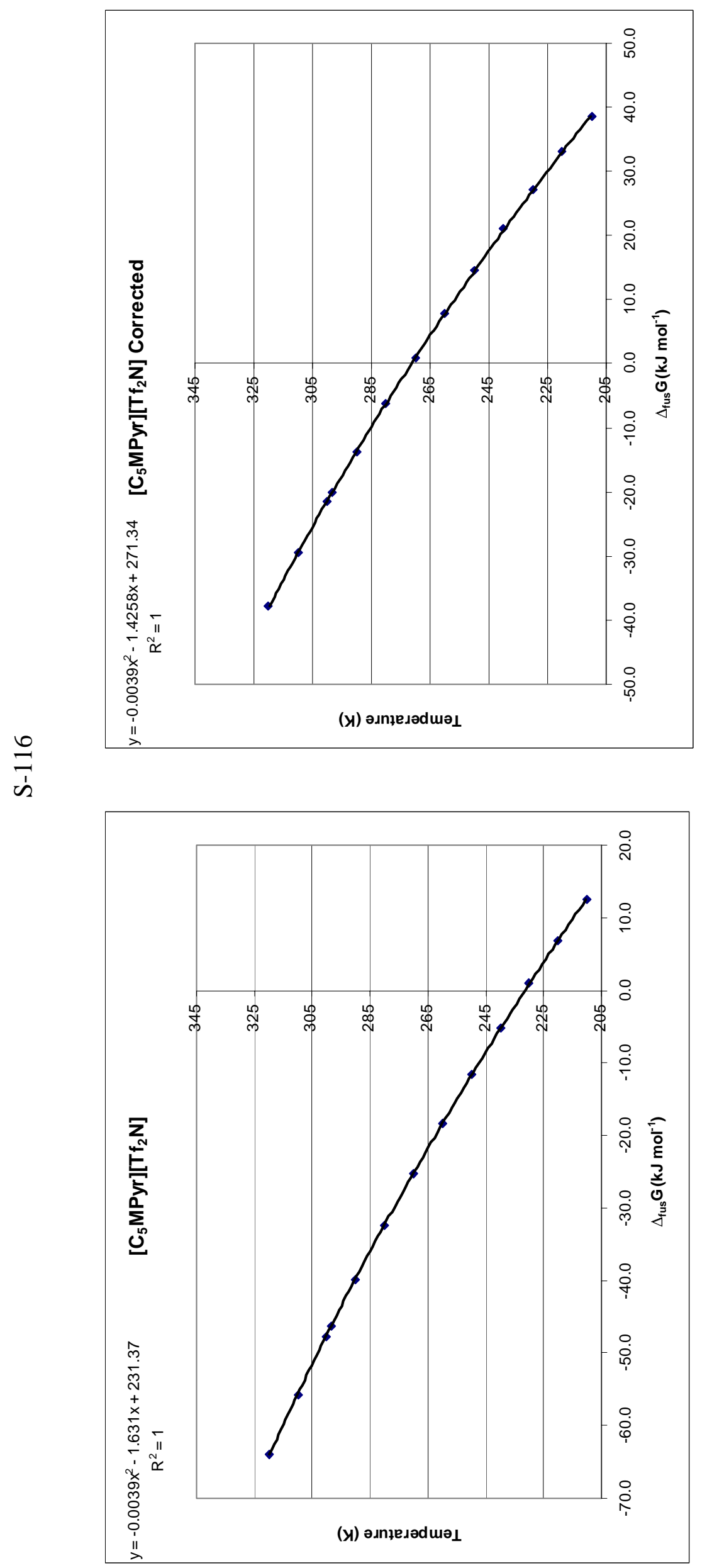


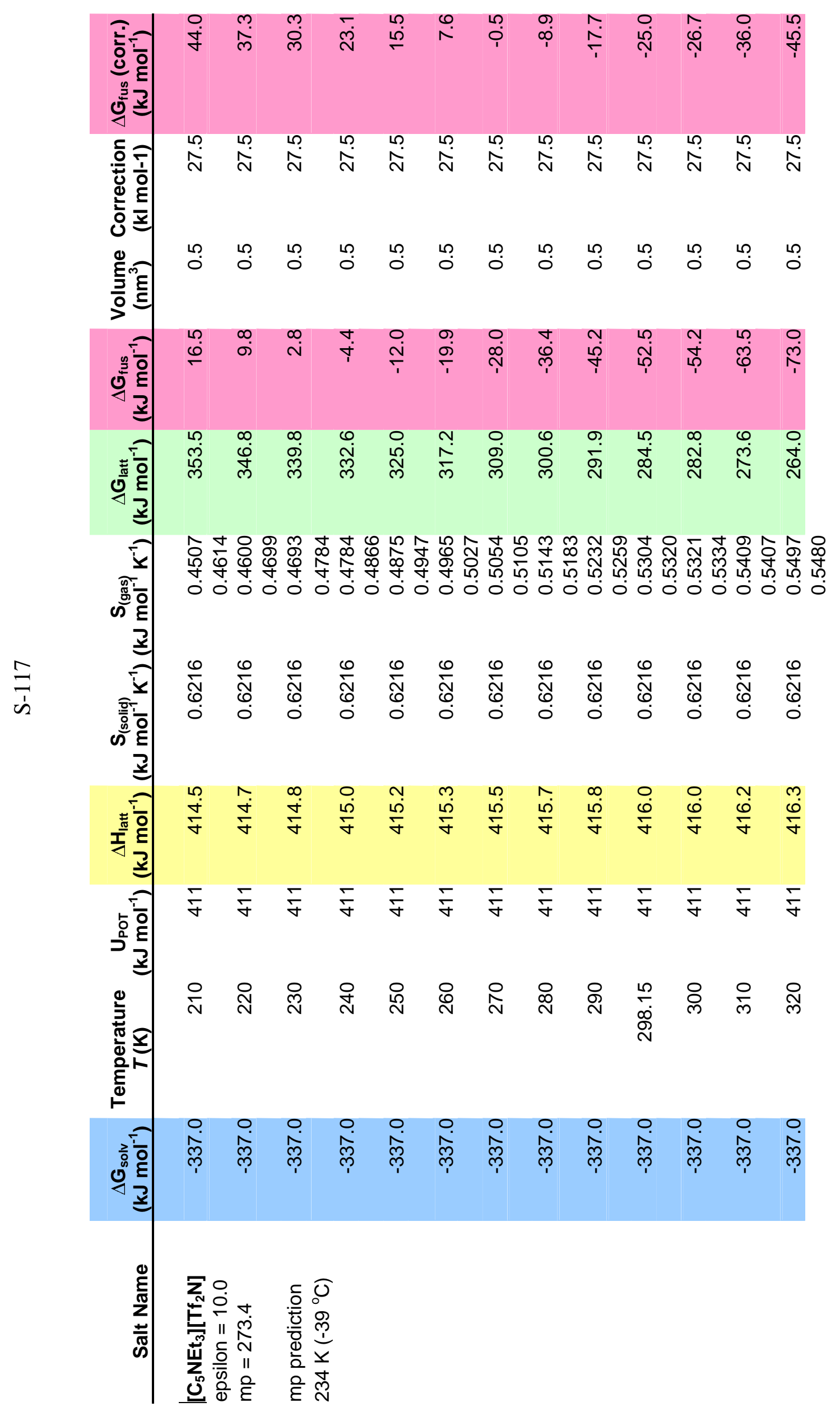




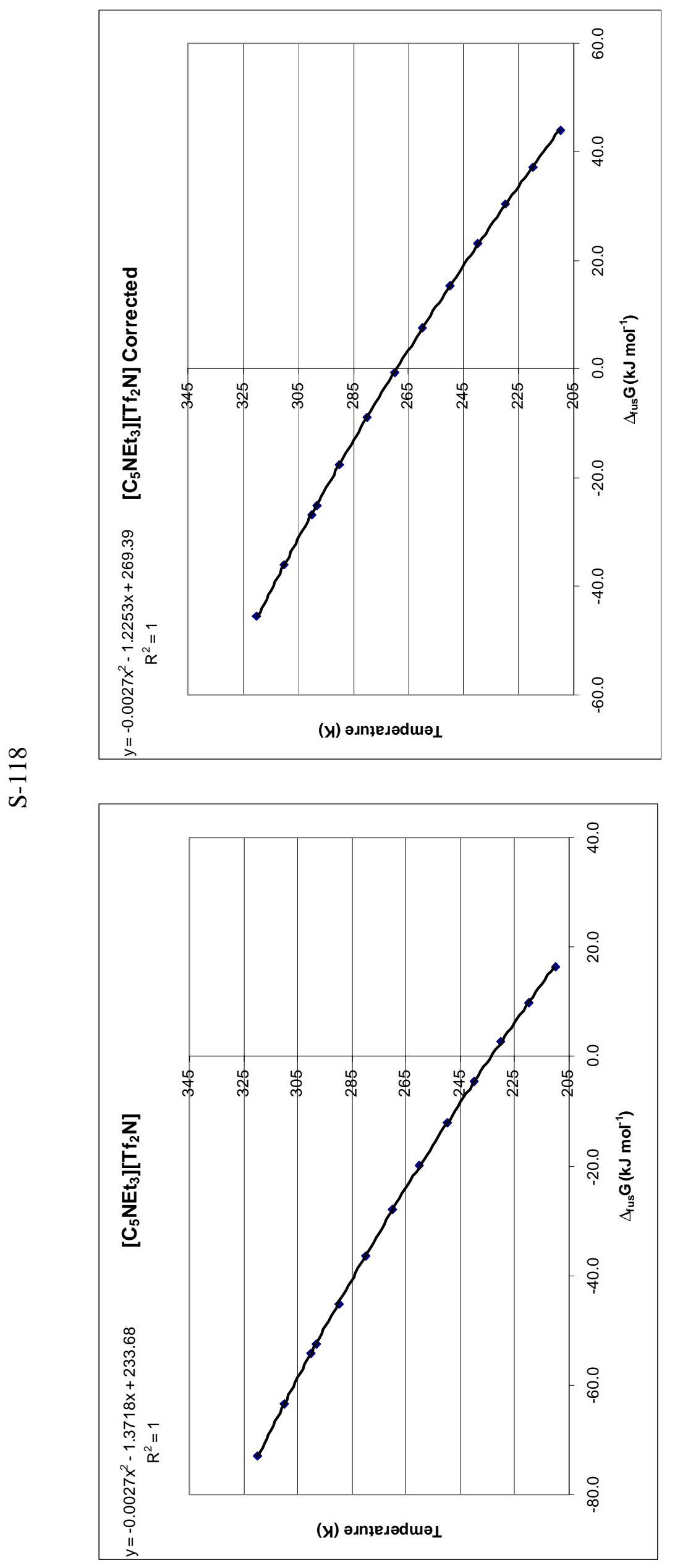




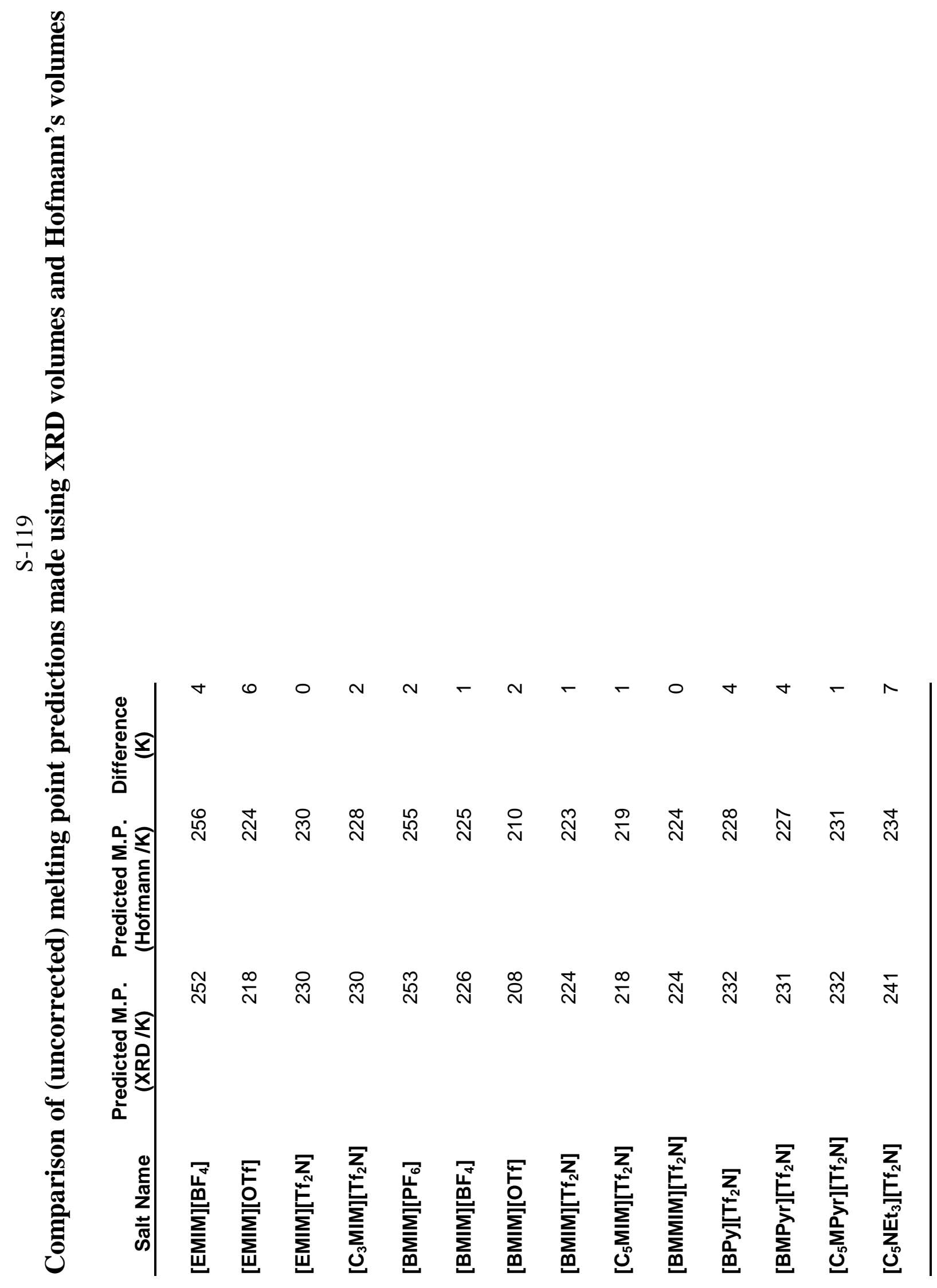




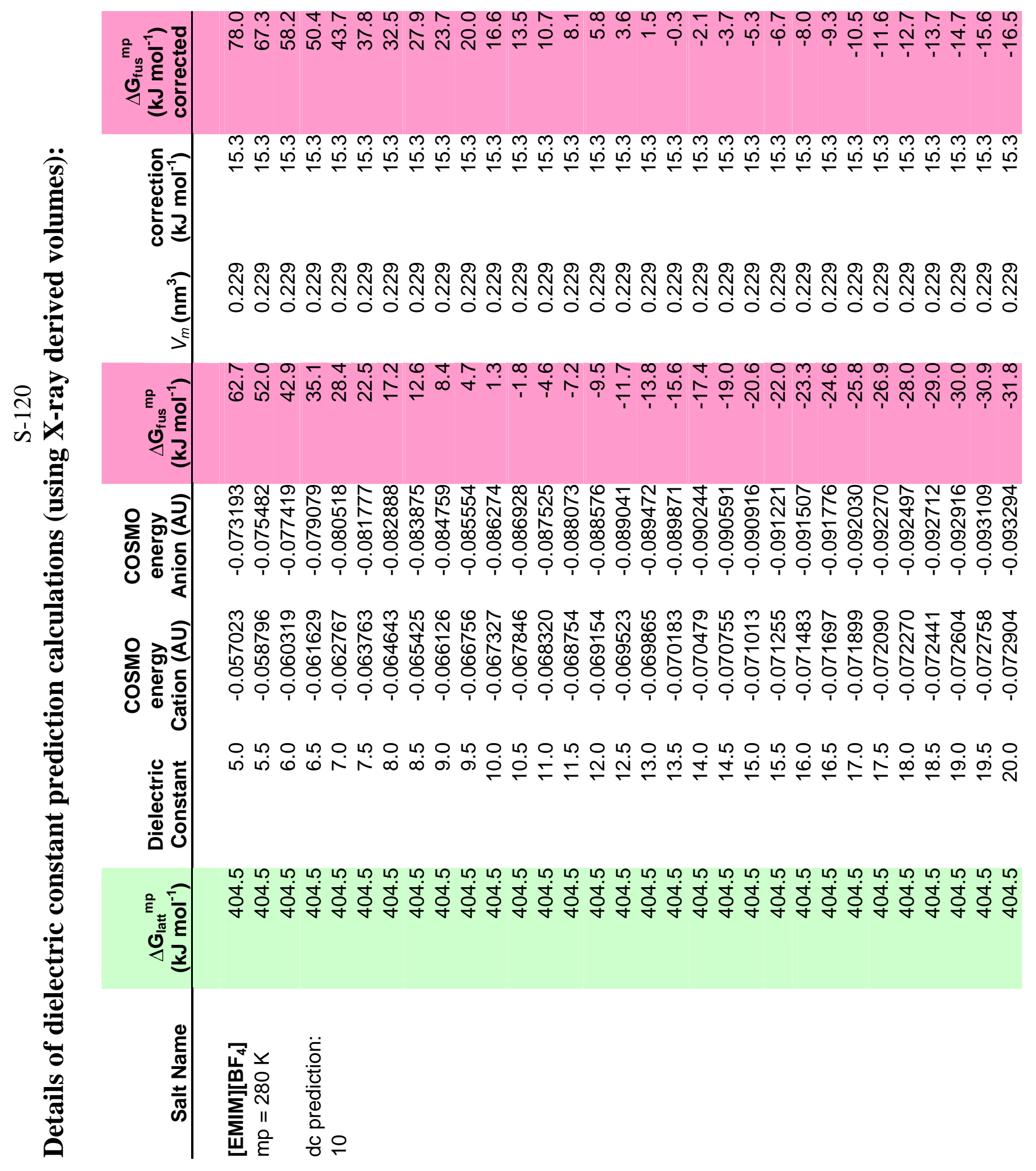




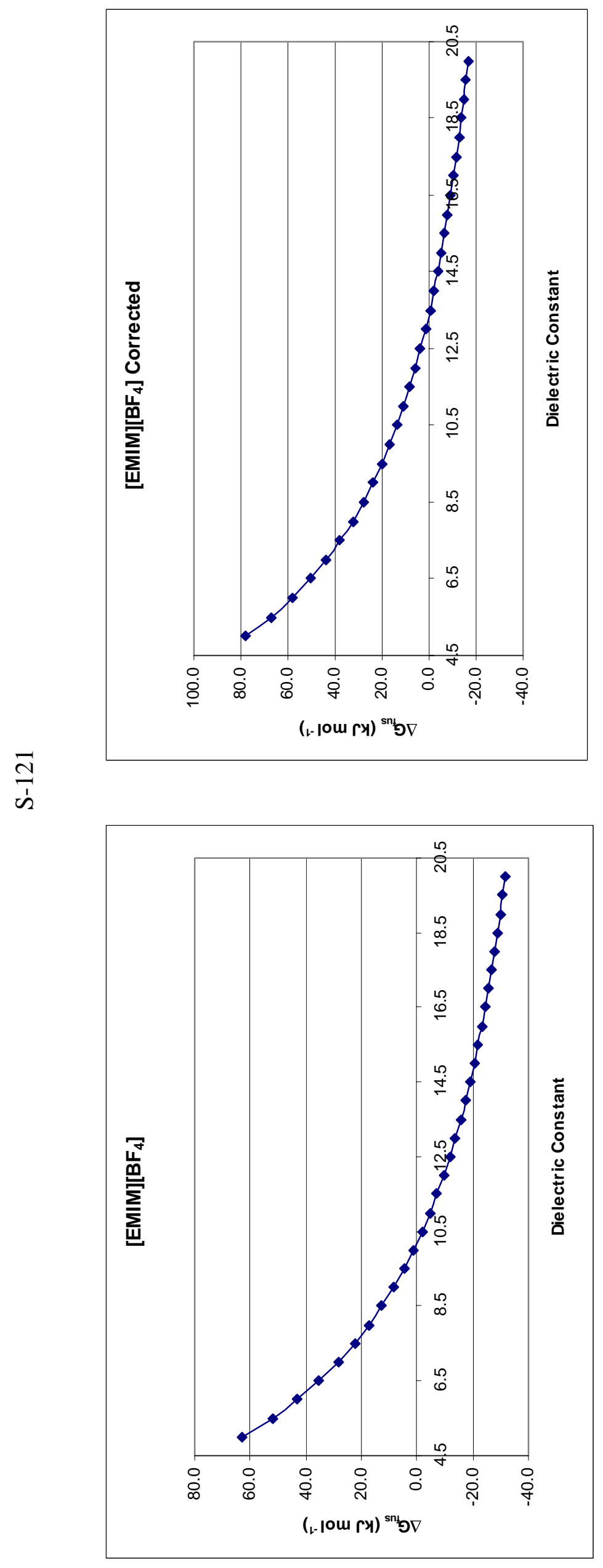




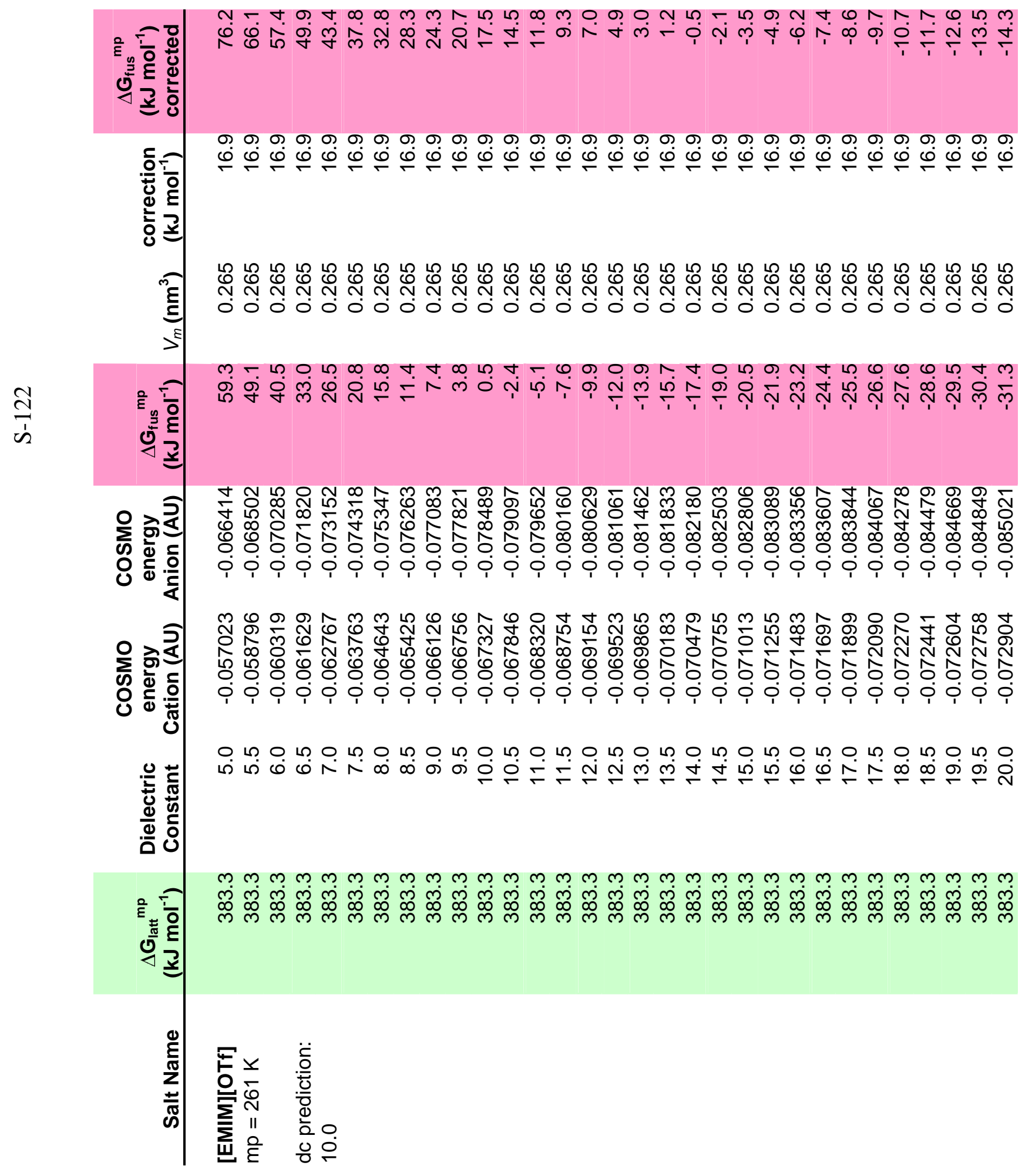




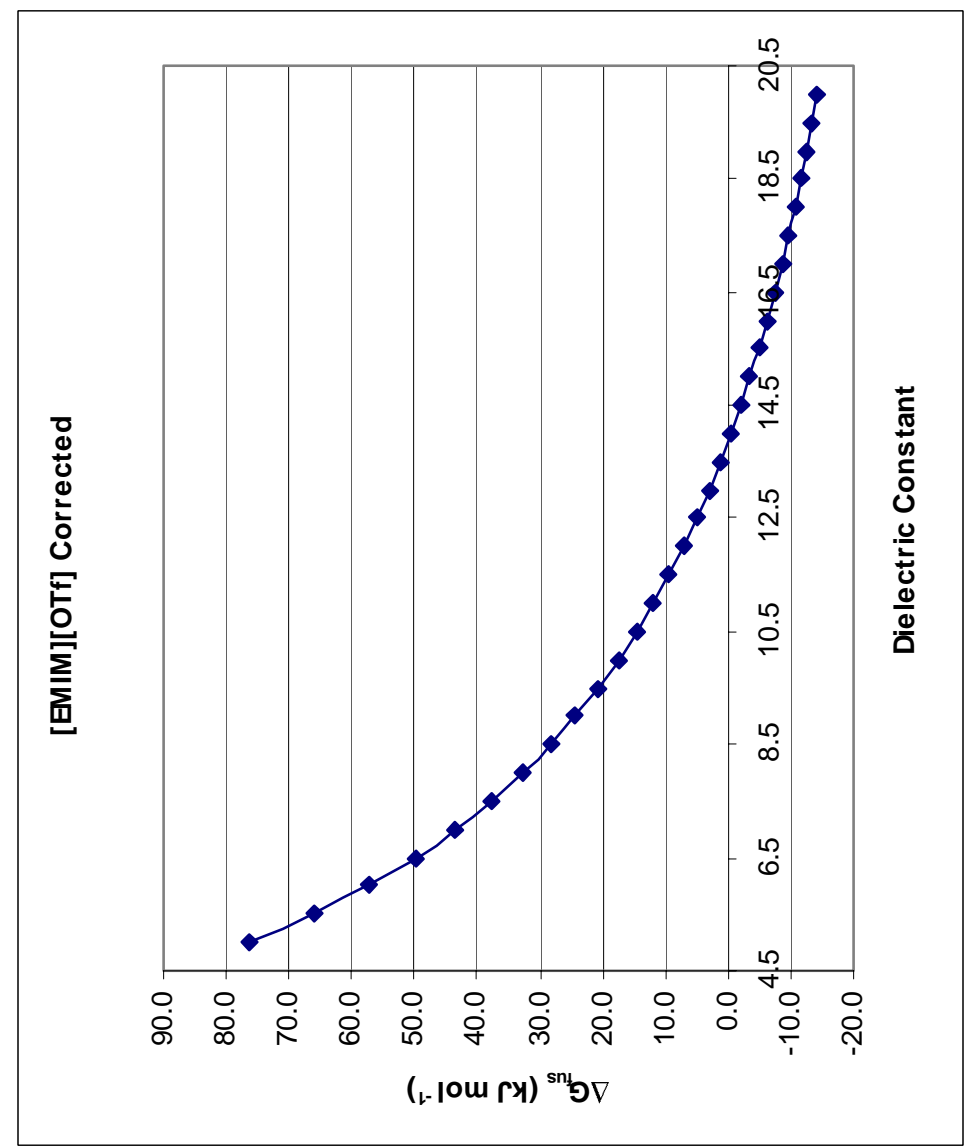

$\frac{n}{n}$

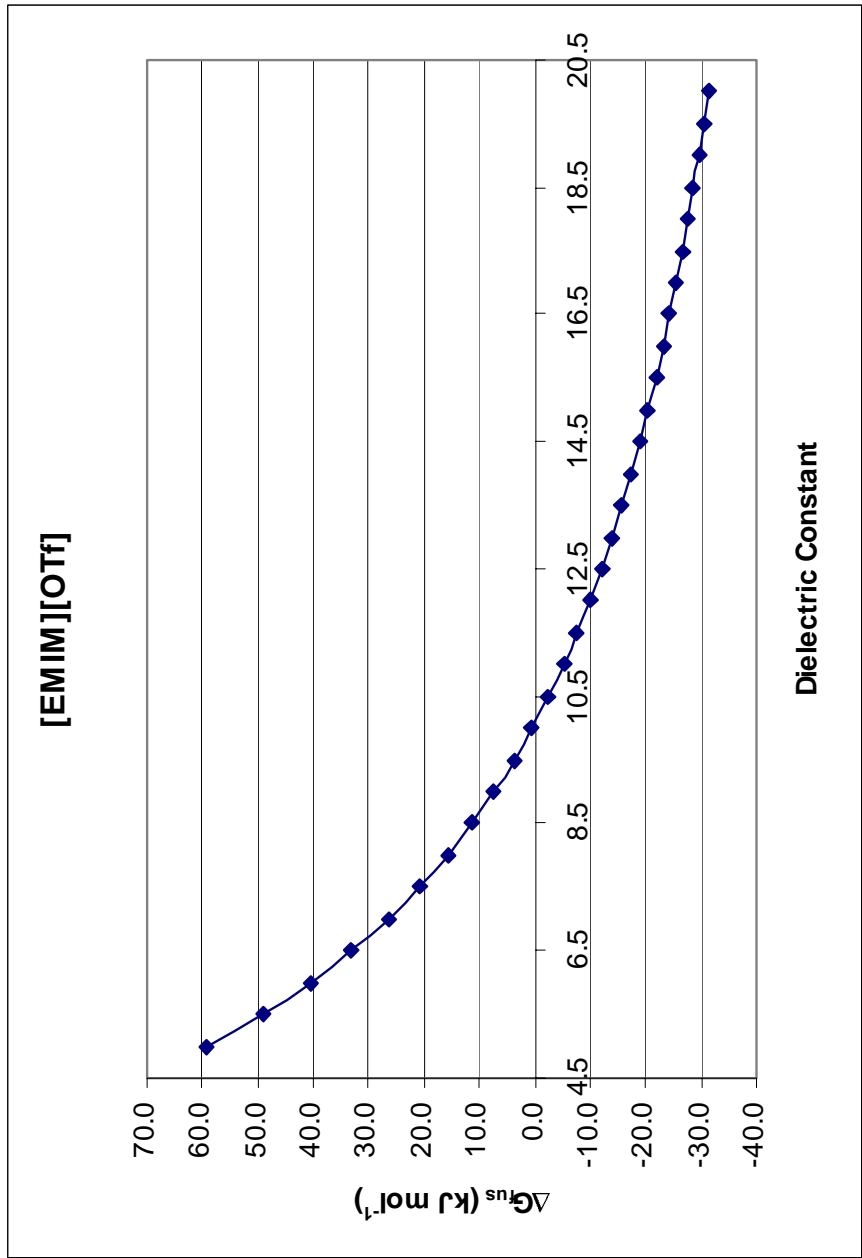




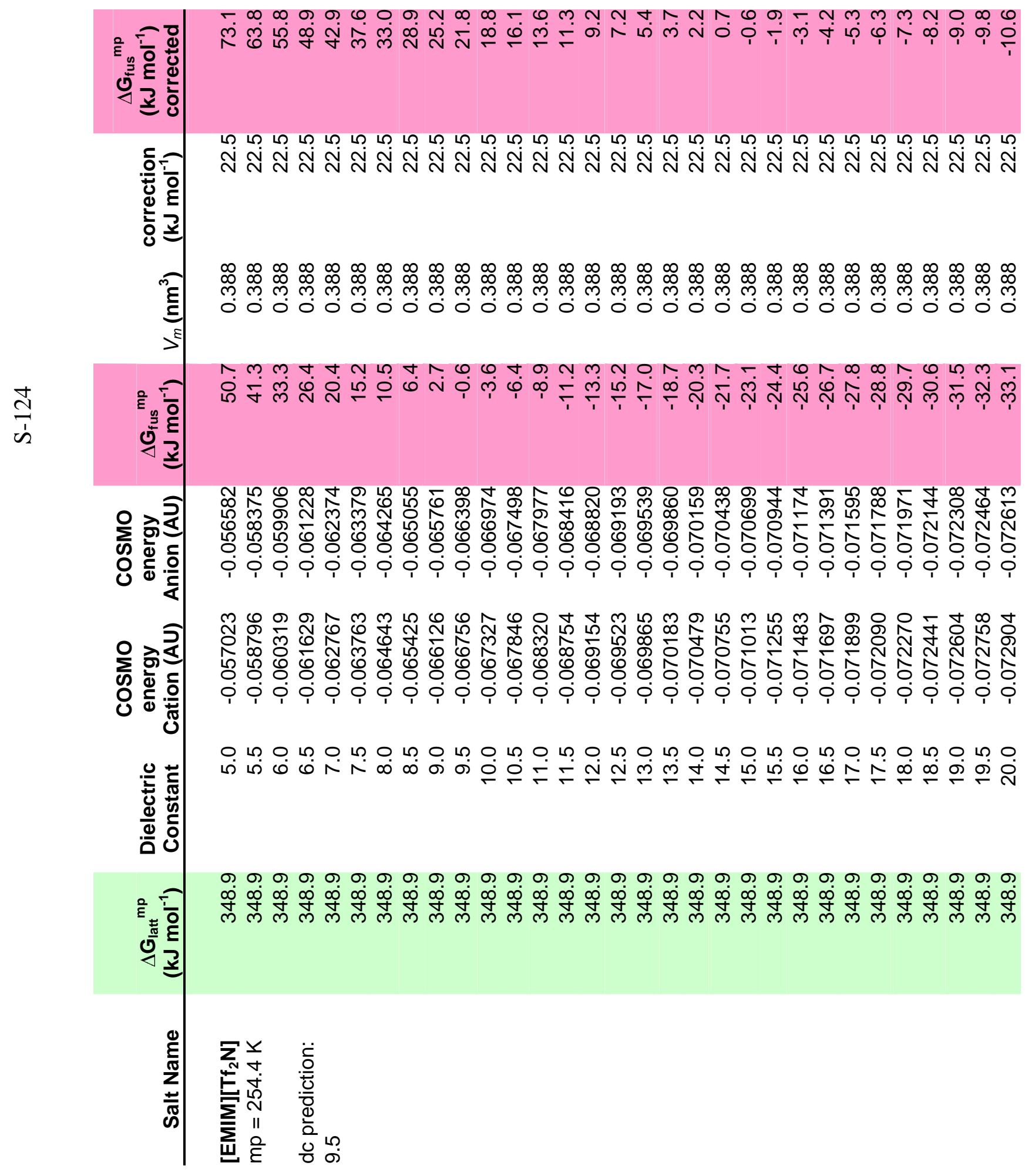


กิ
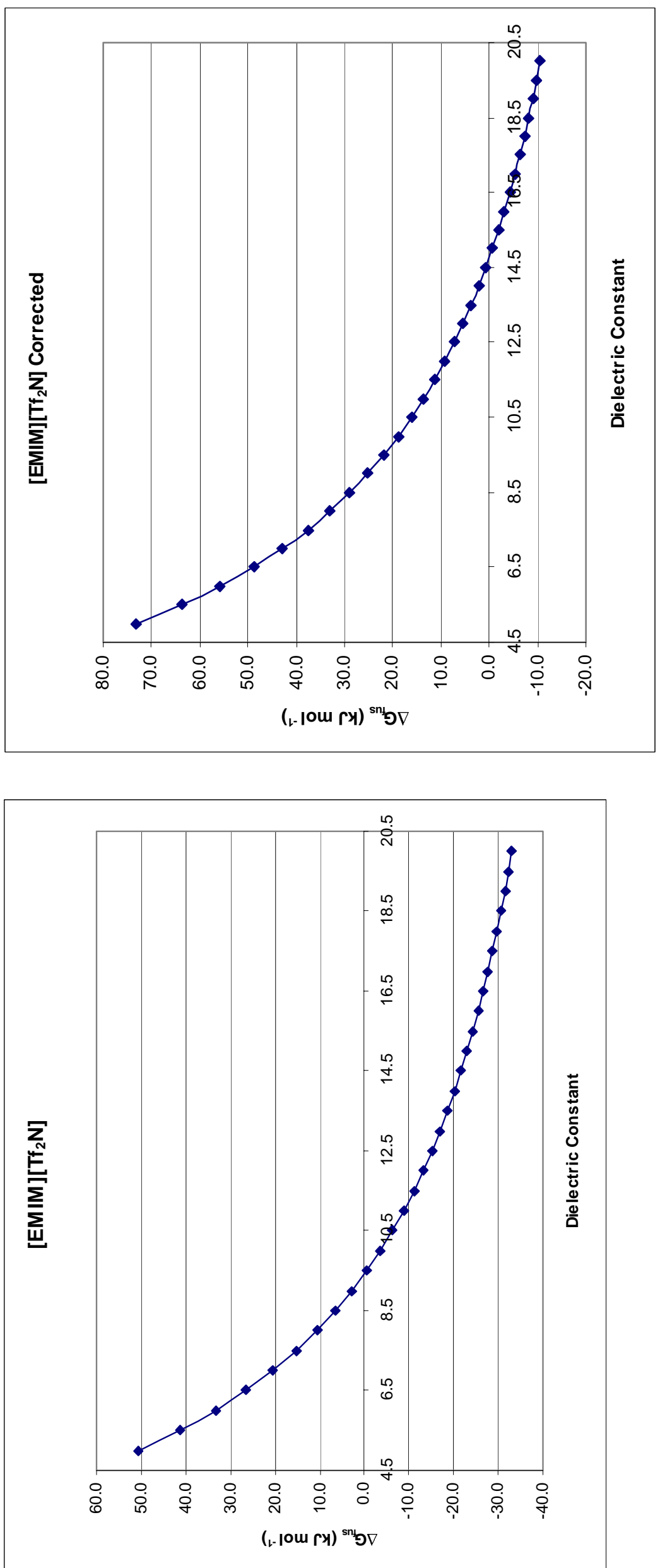


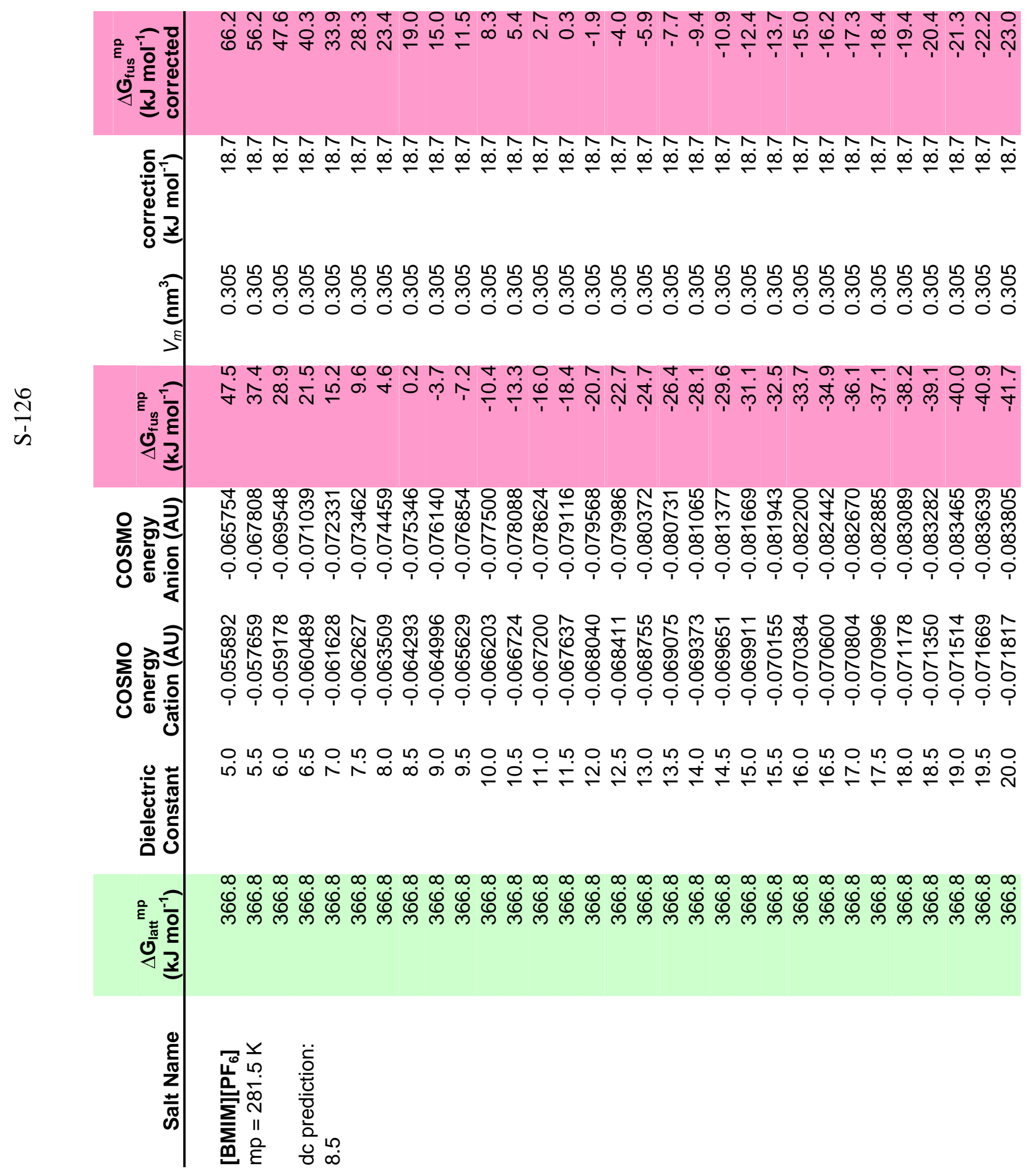


$\frac{\hat{v}}{i}$
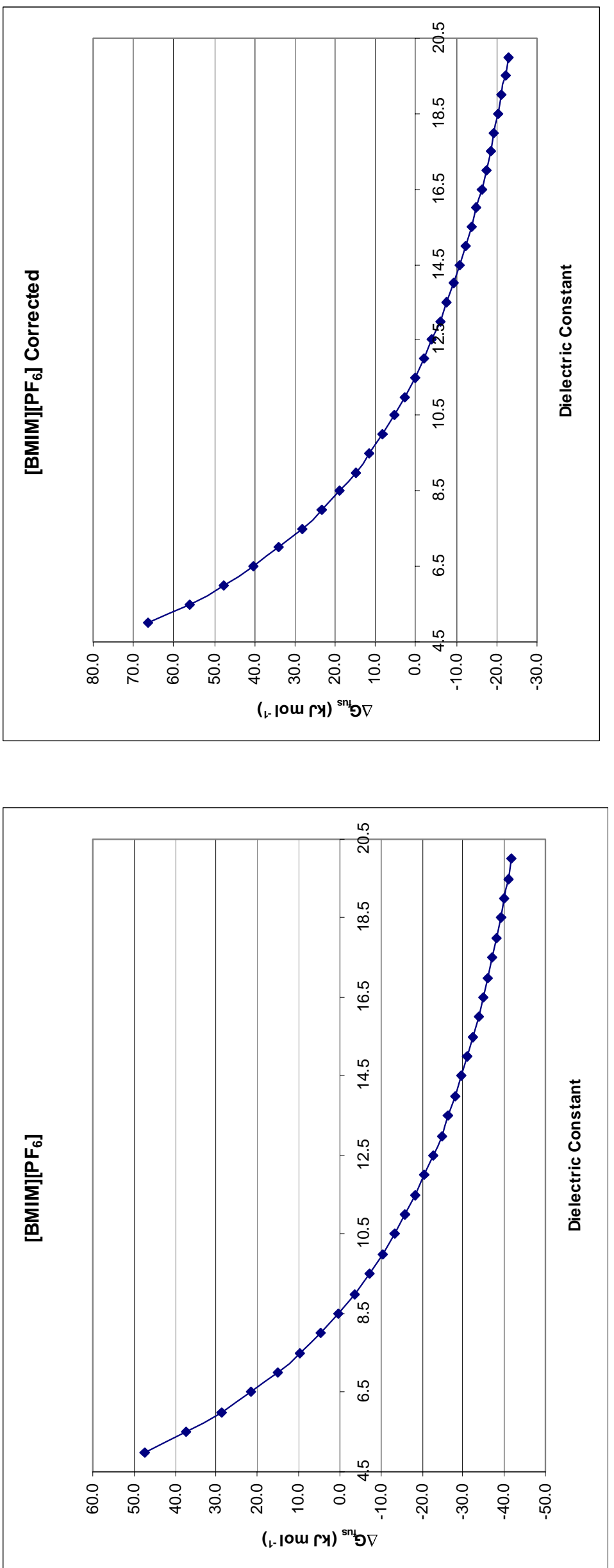


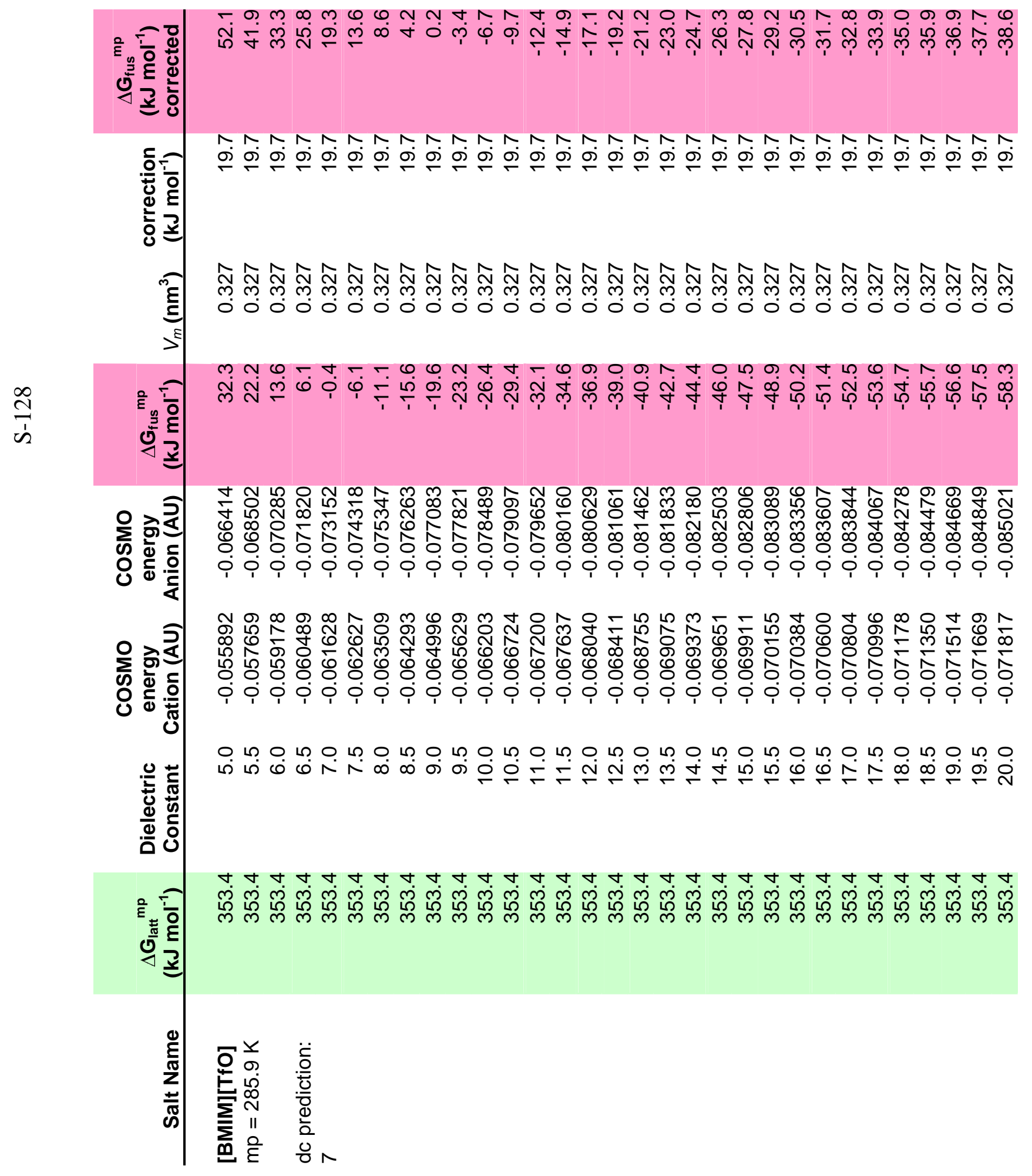


$\frac{a}{i s}$
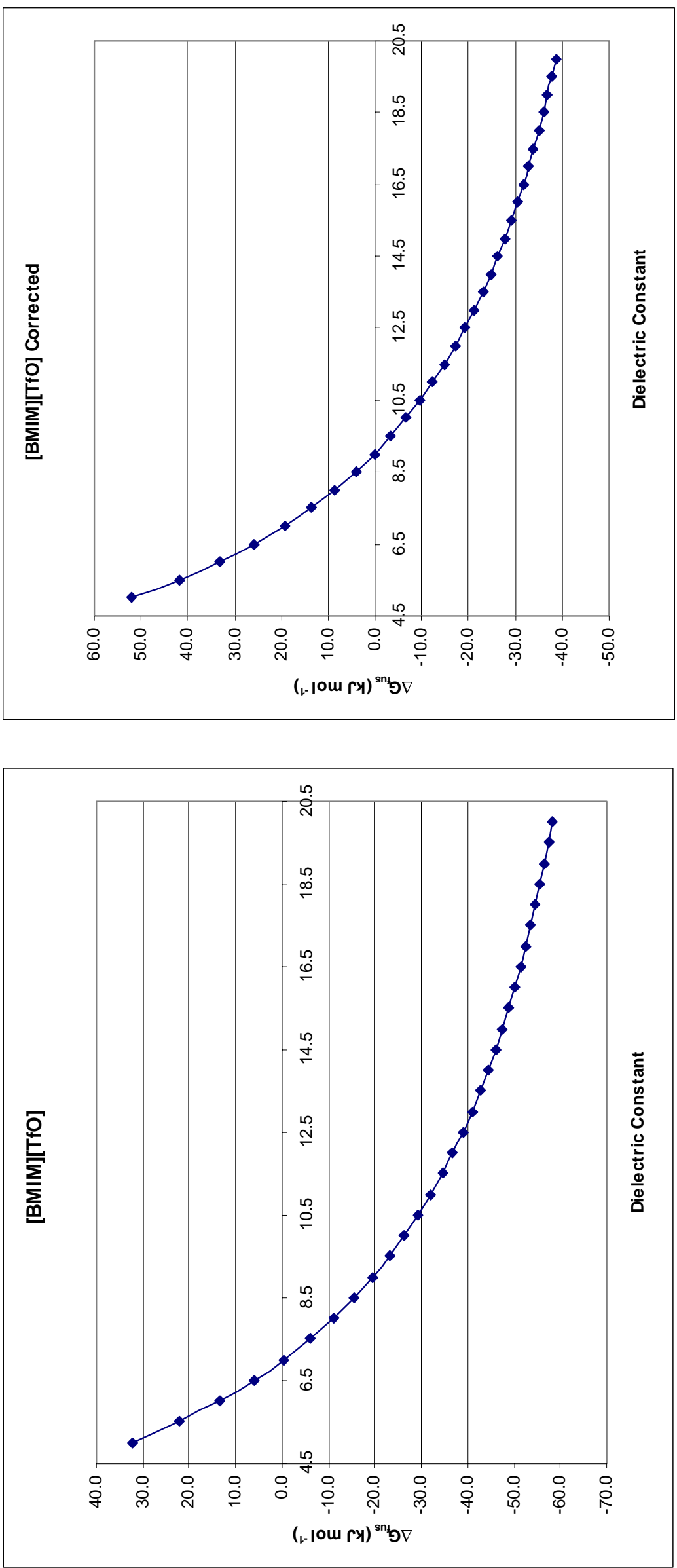


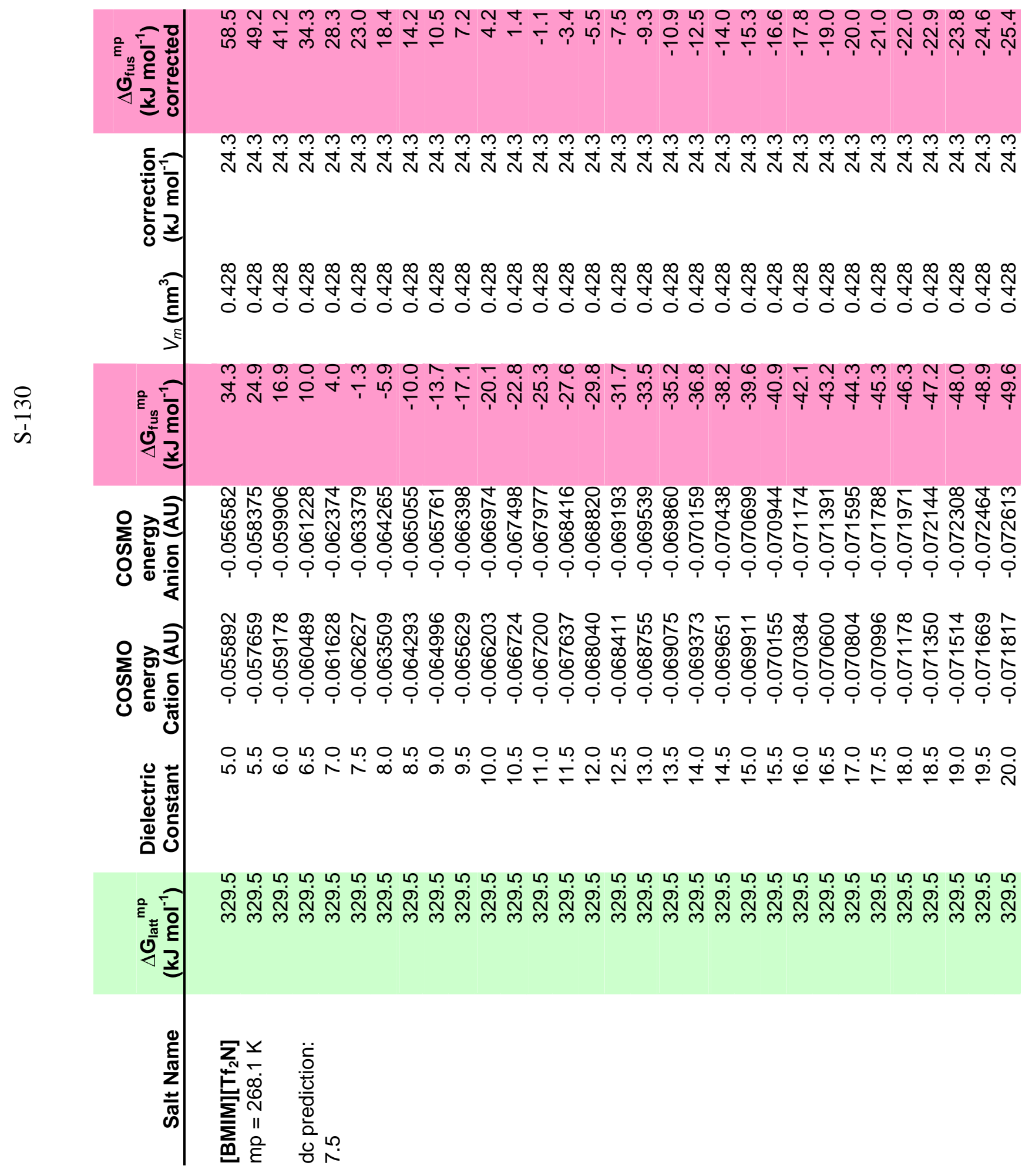




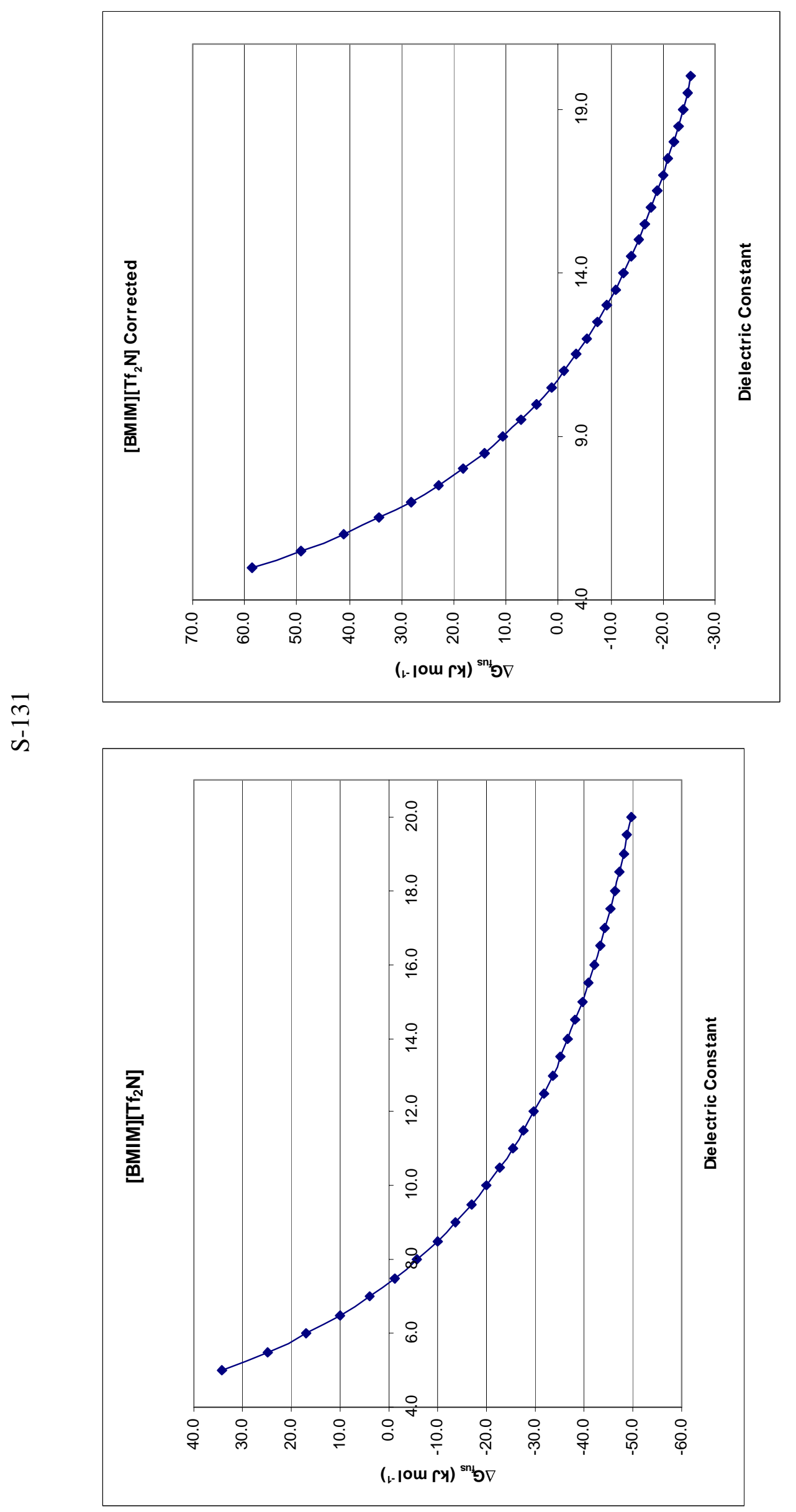




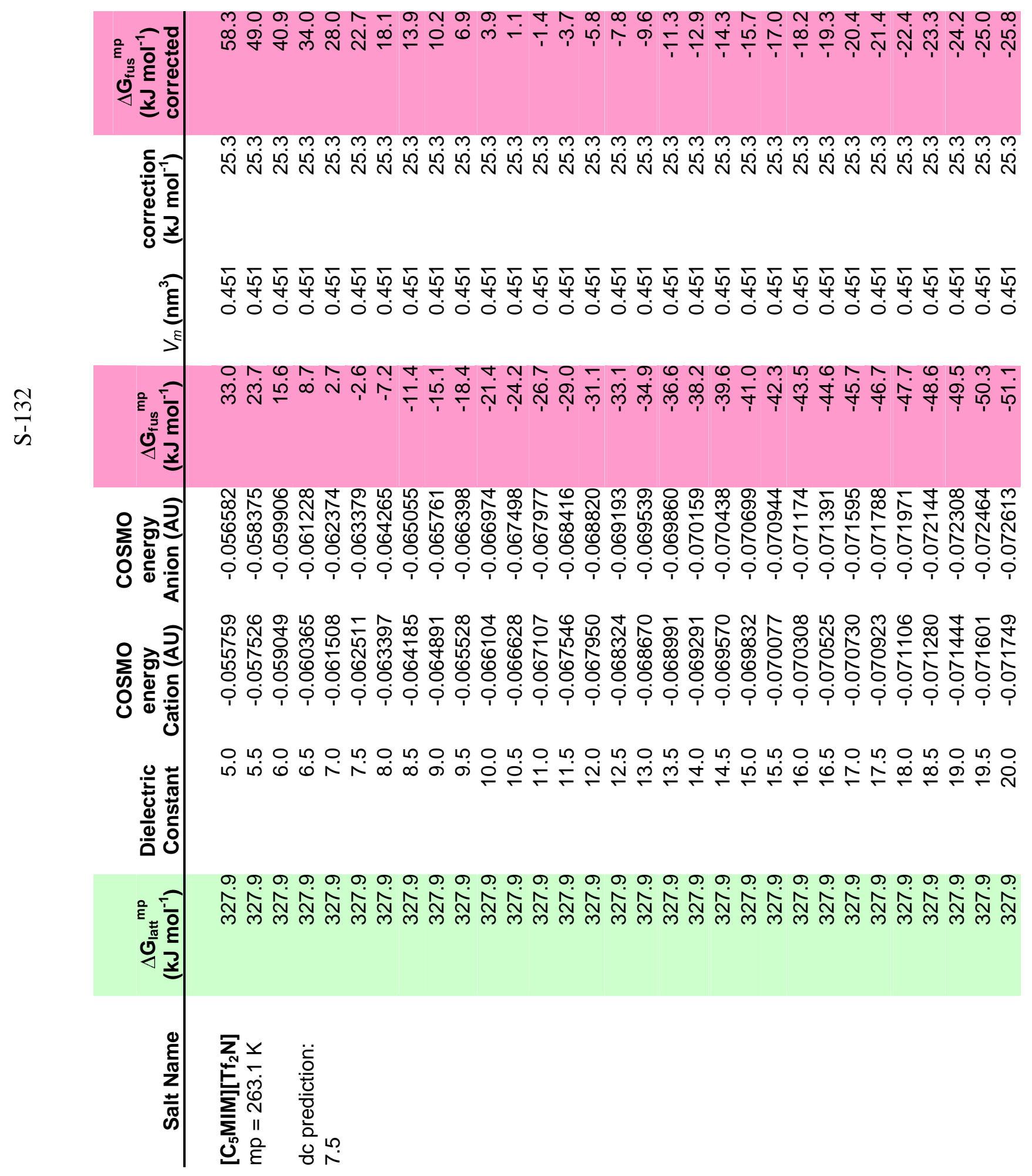


$\frac{m}{n}$
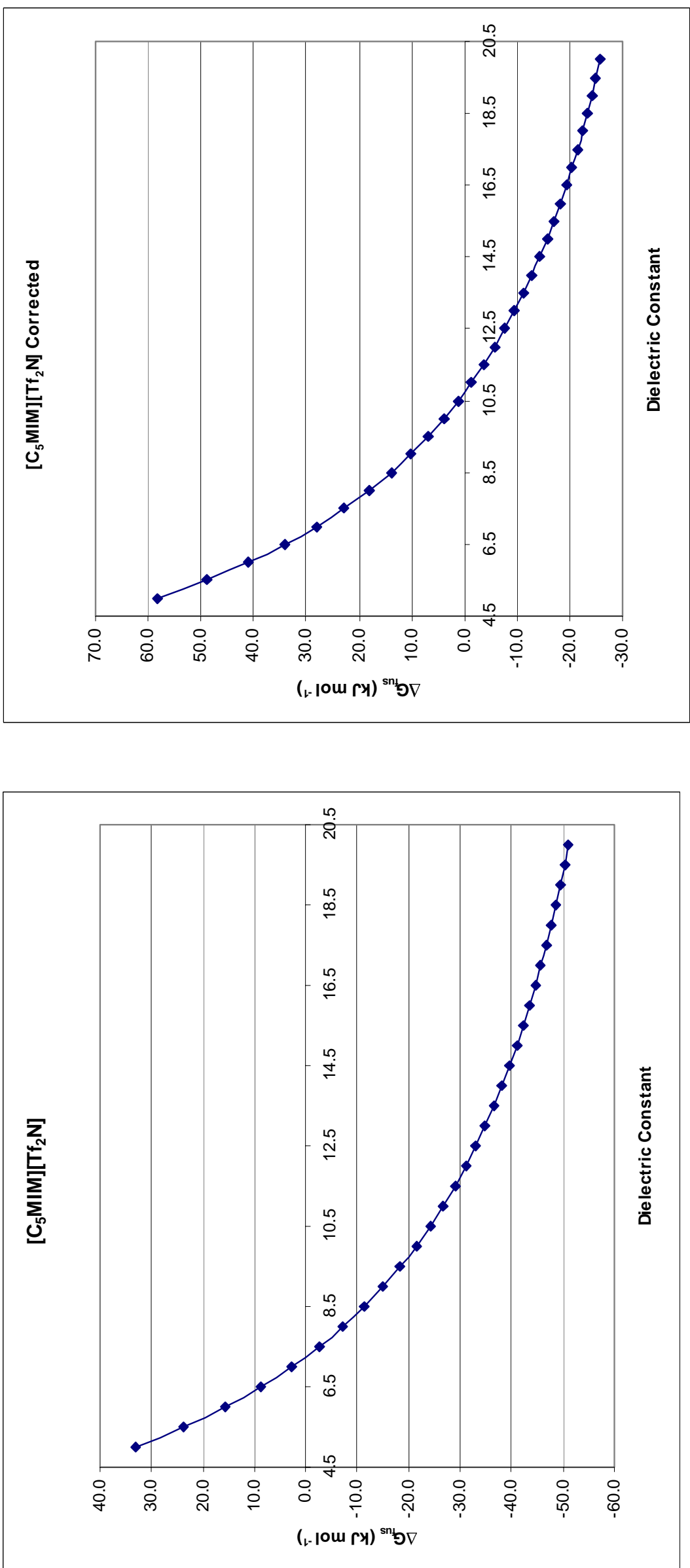


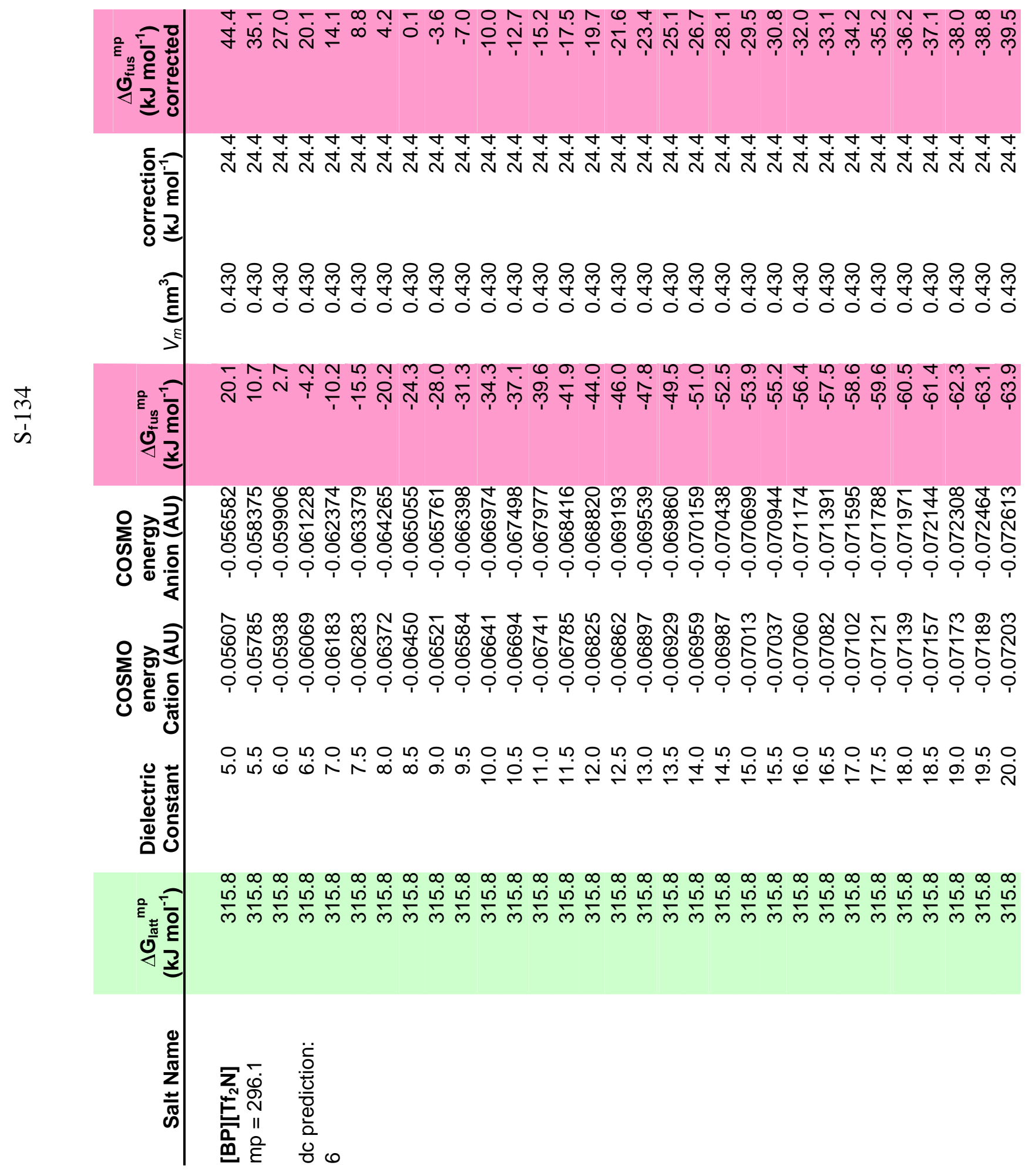




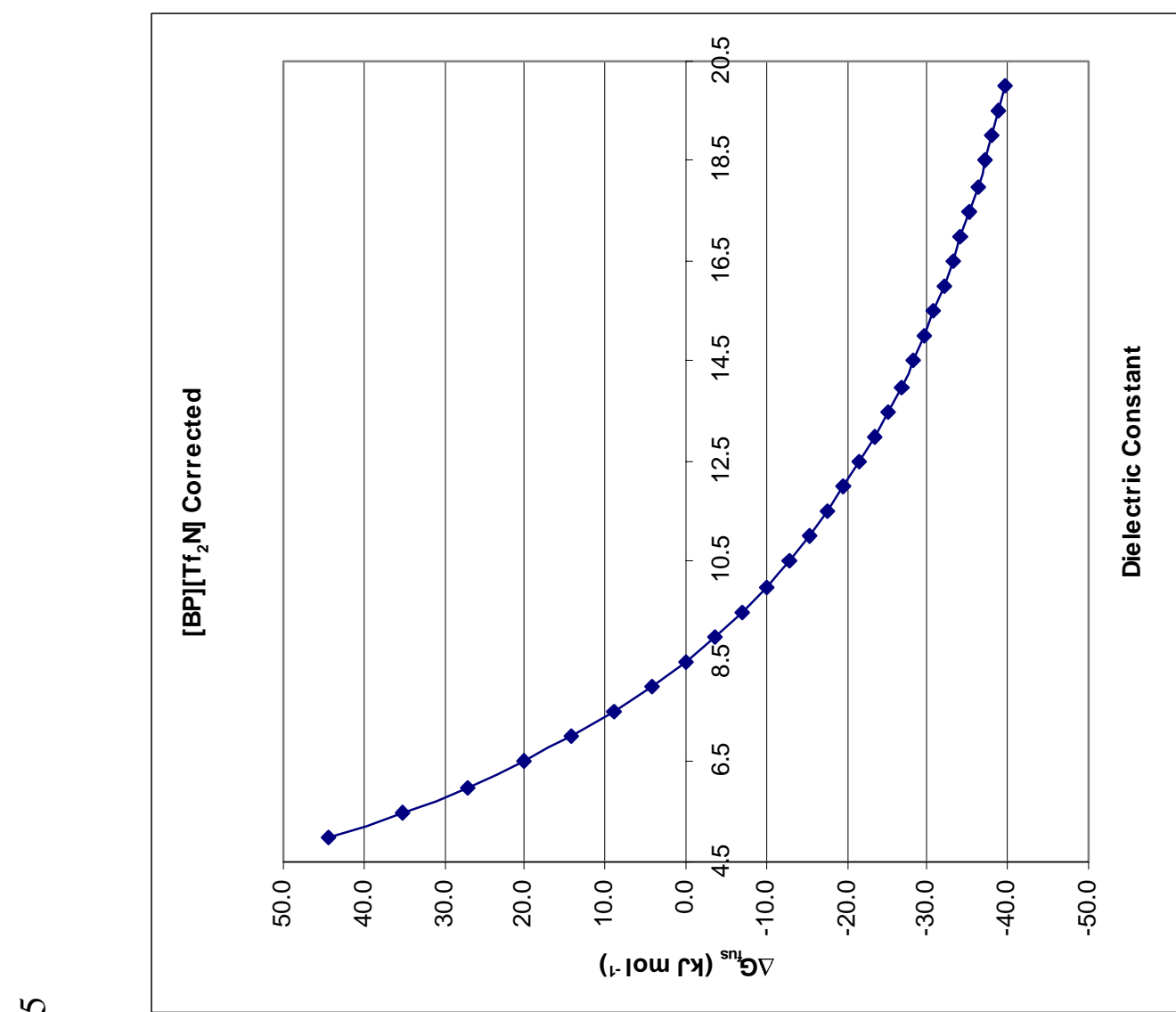

$\frac{n}{n}$

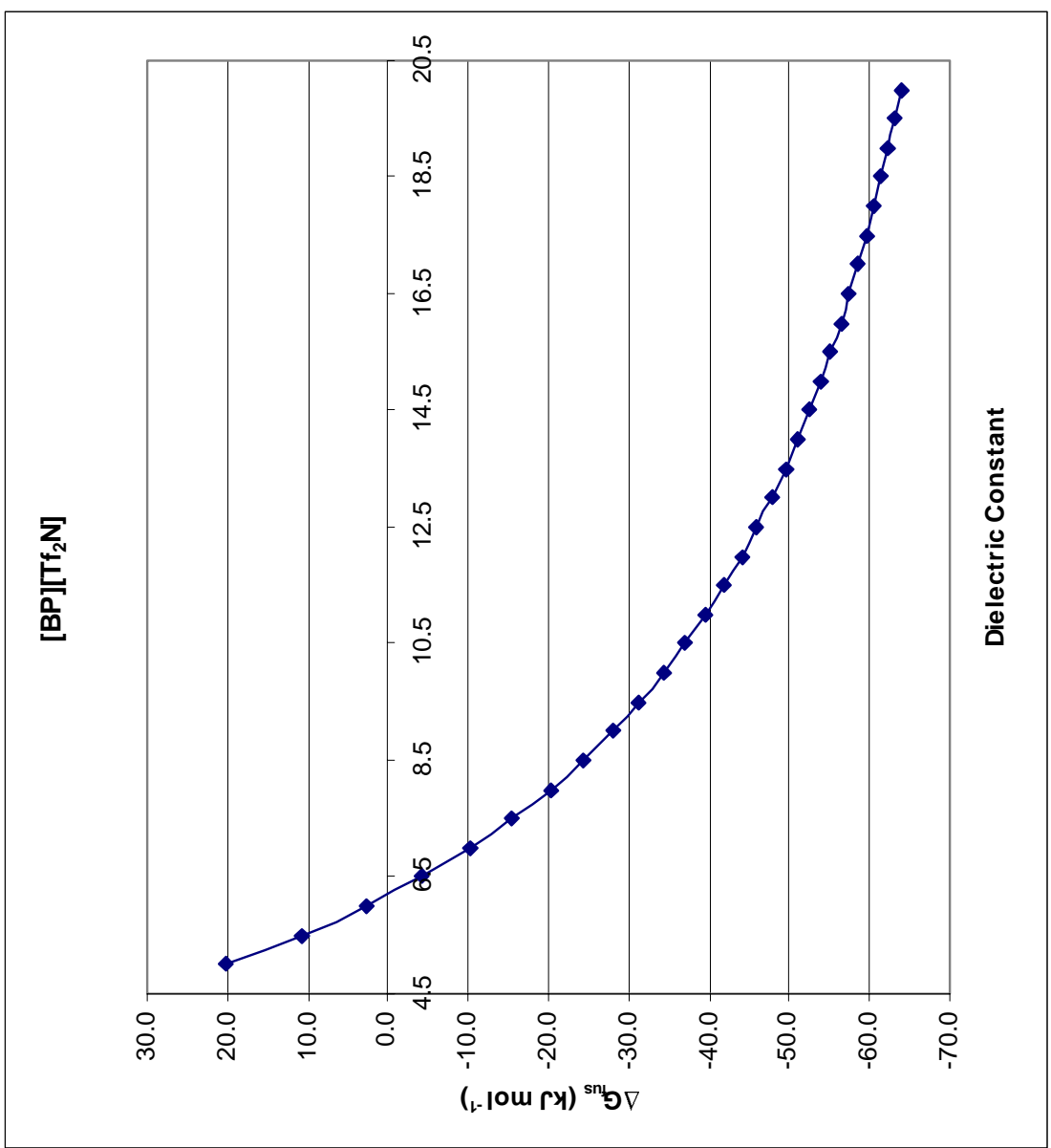




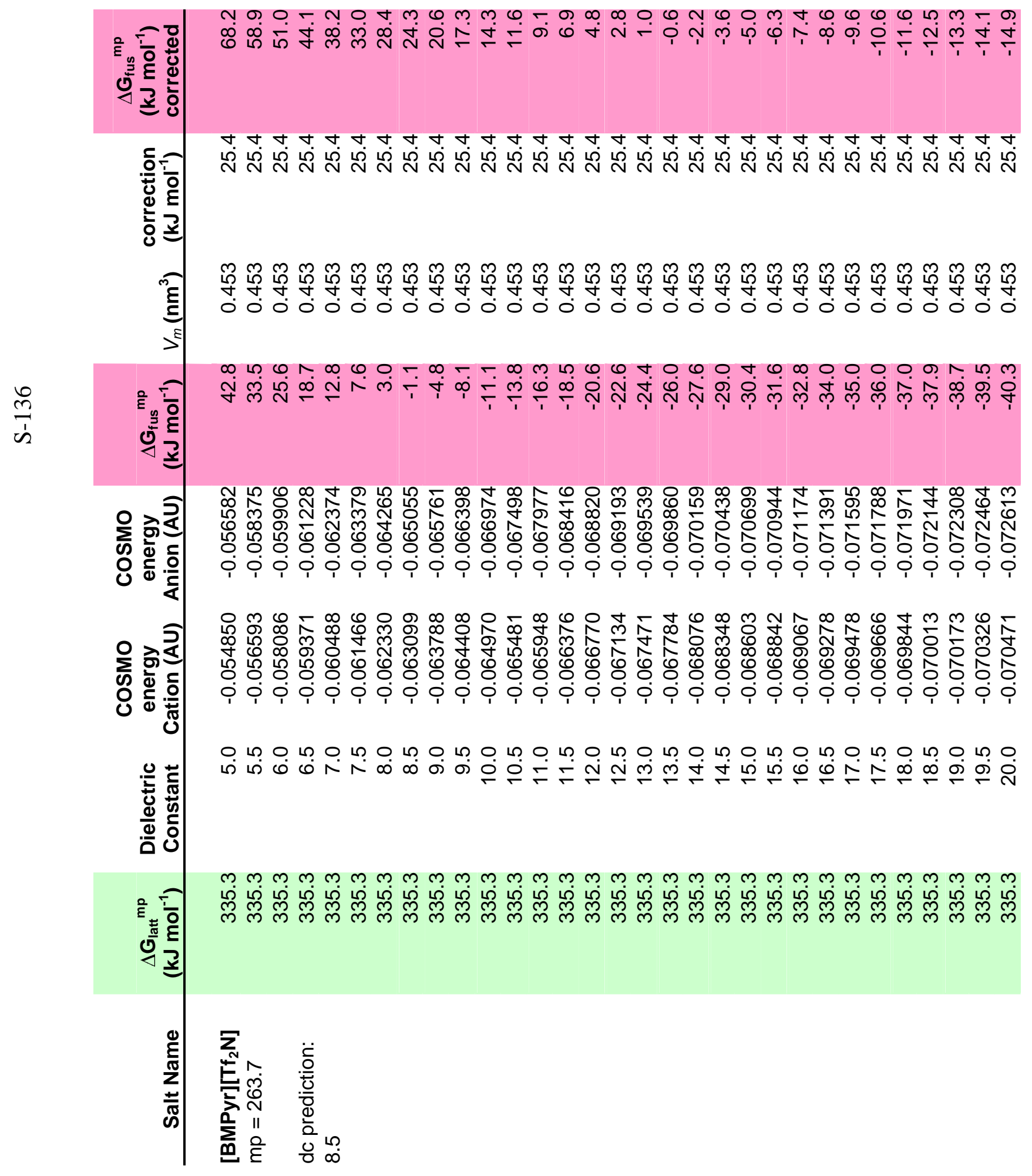




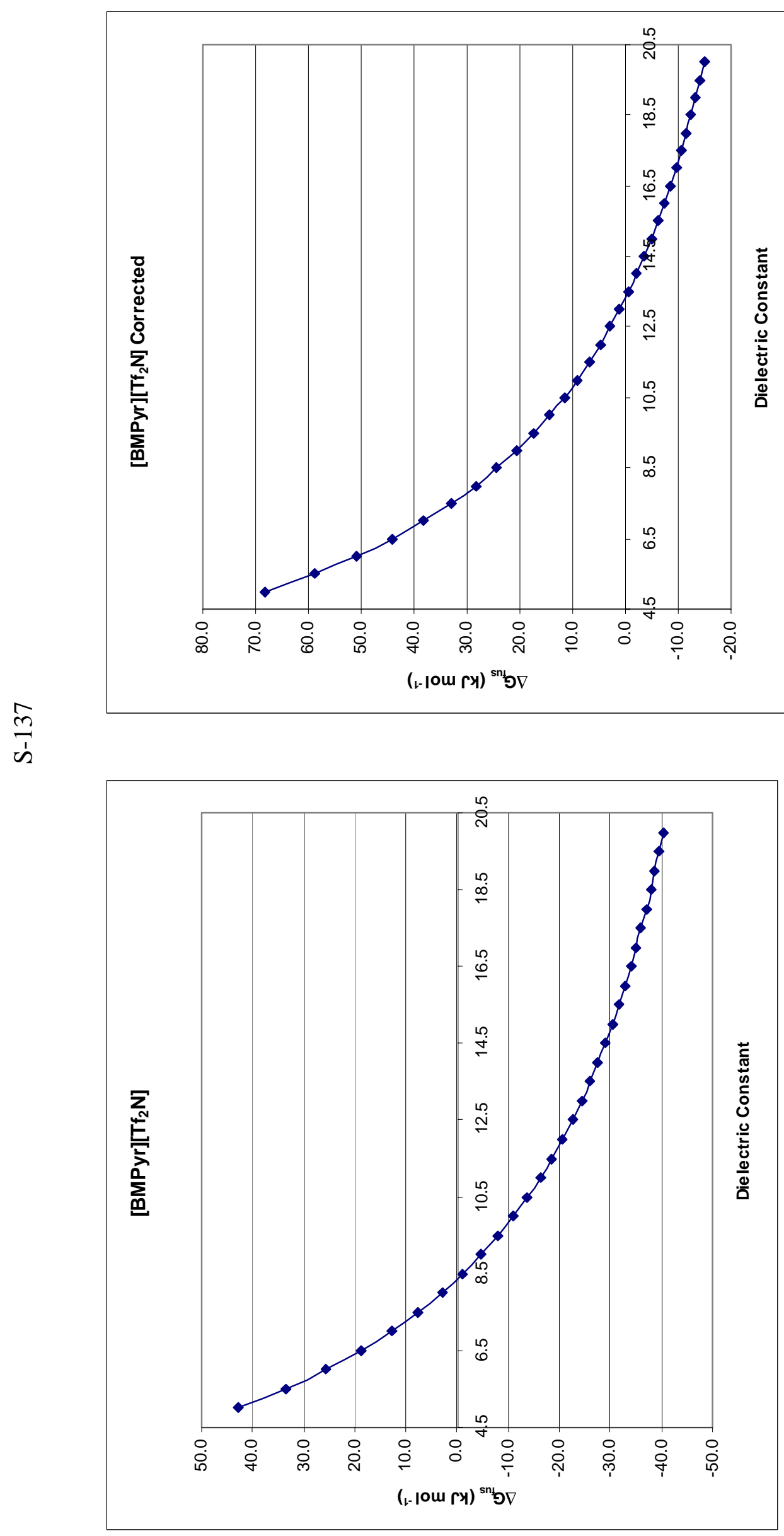




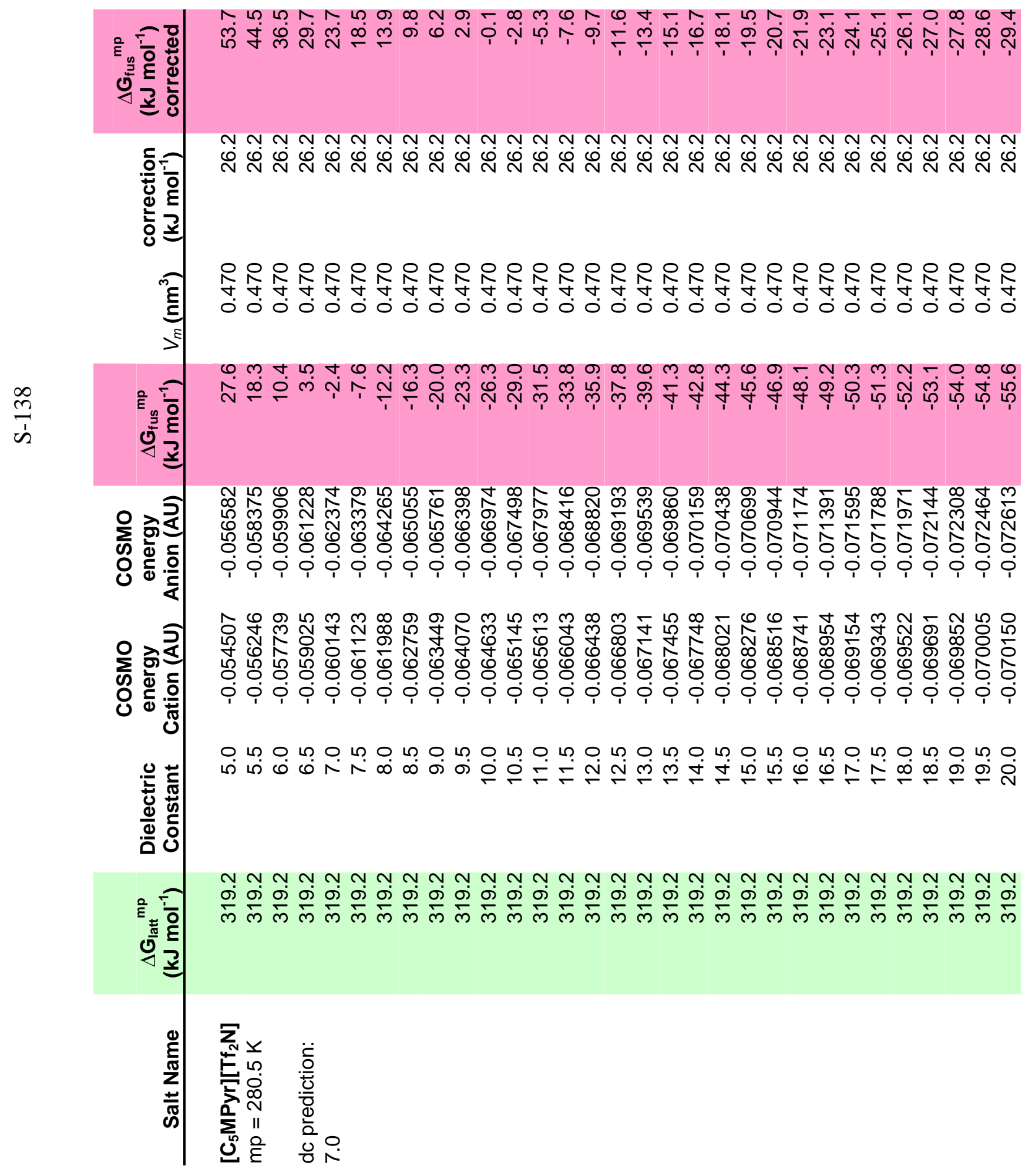




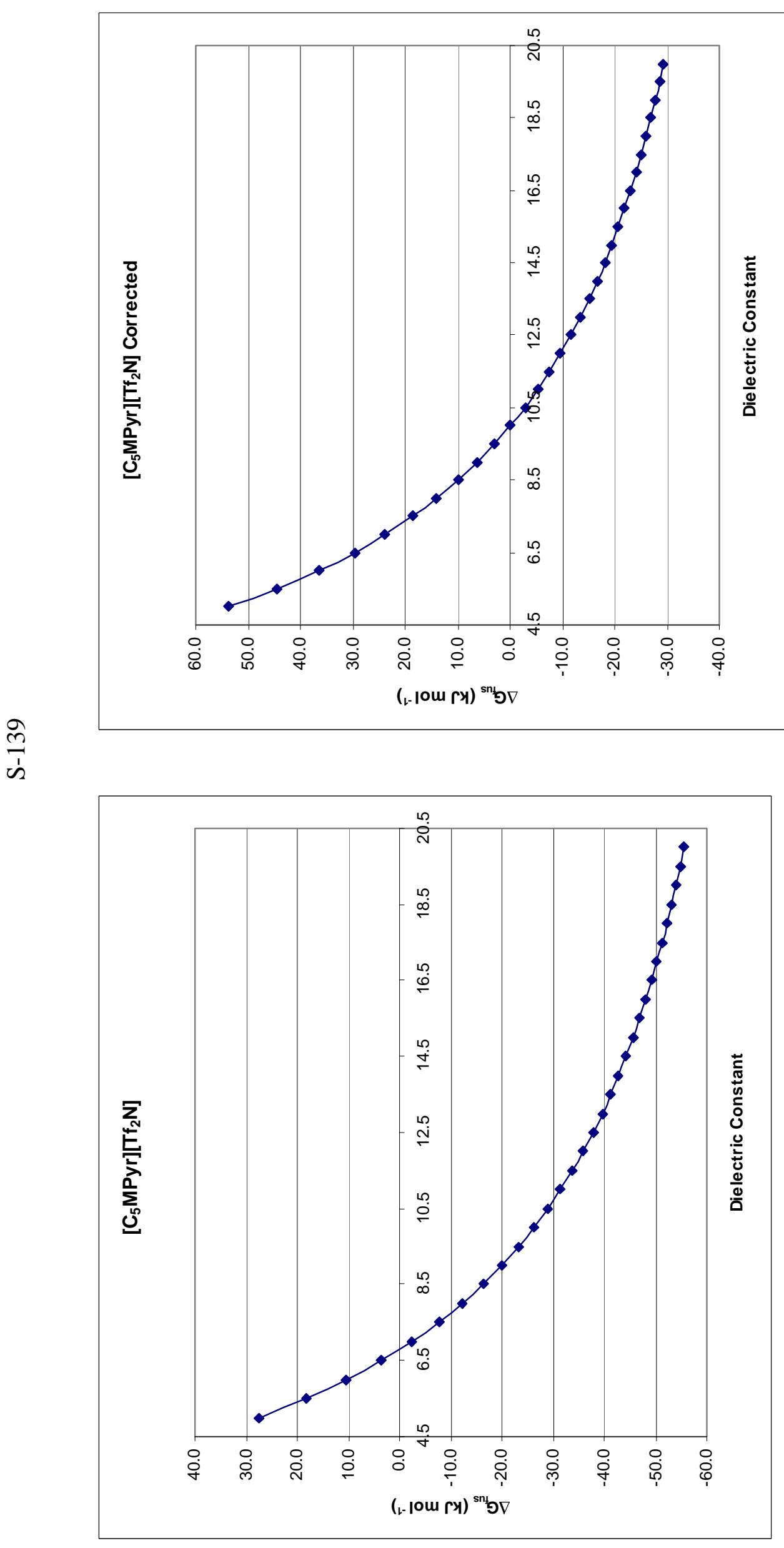




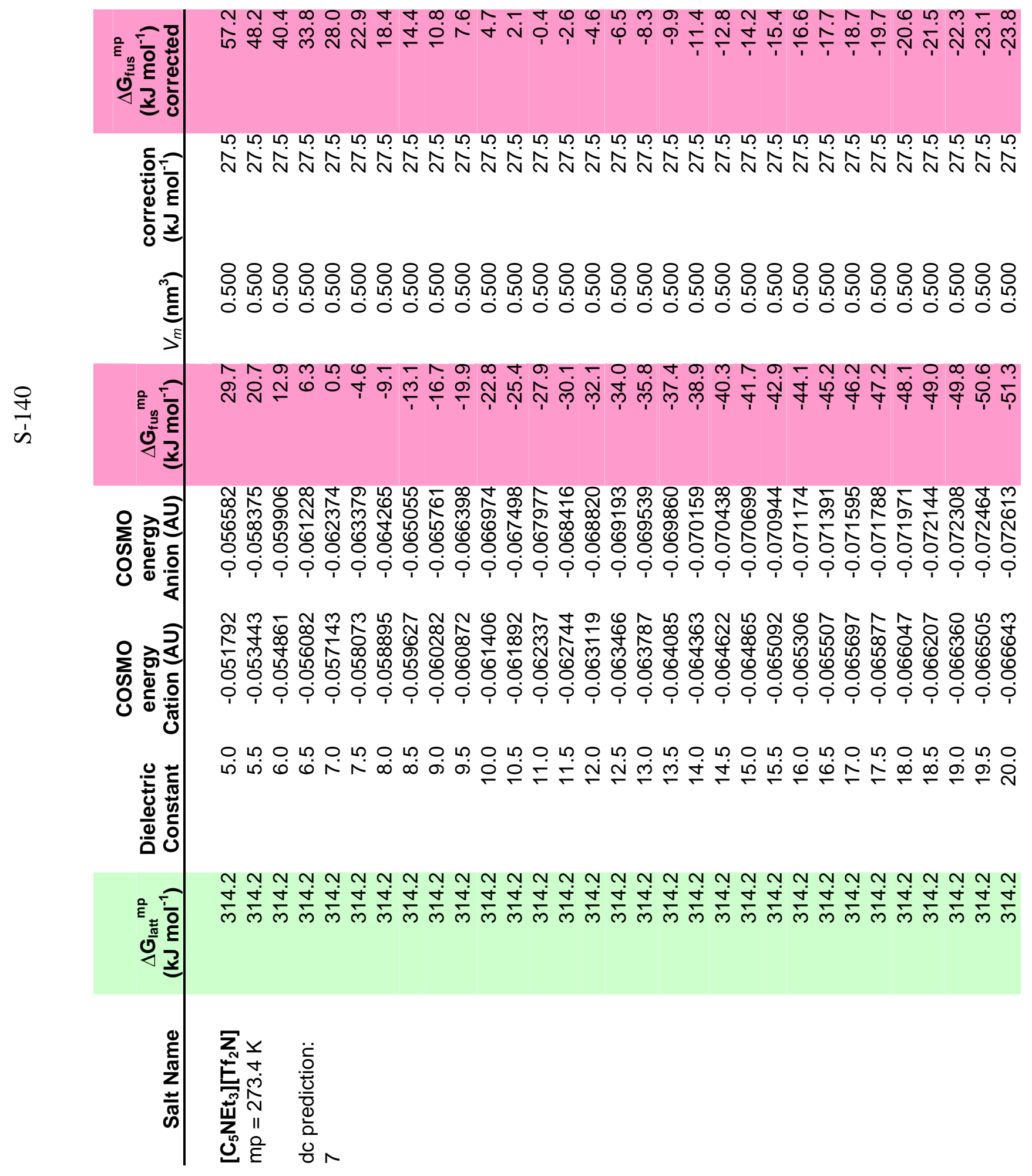




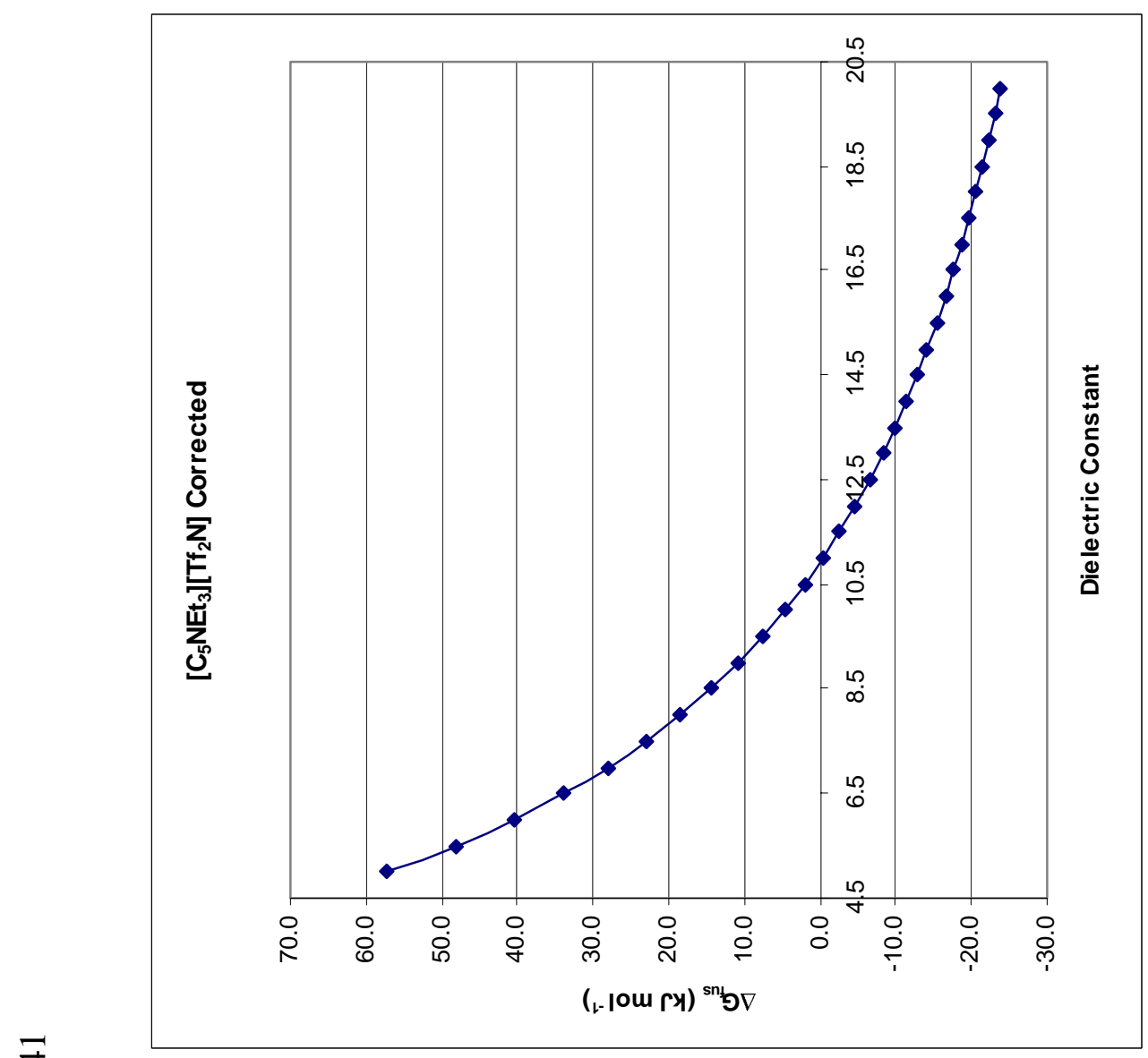

$\frac{\bar{J}}{\dot{s}}$

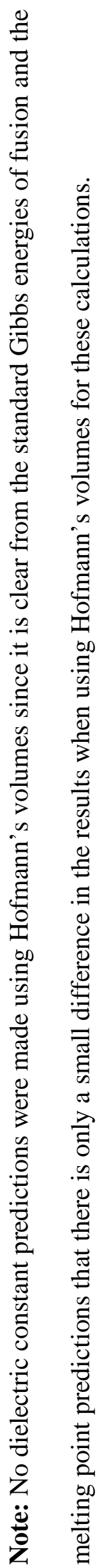




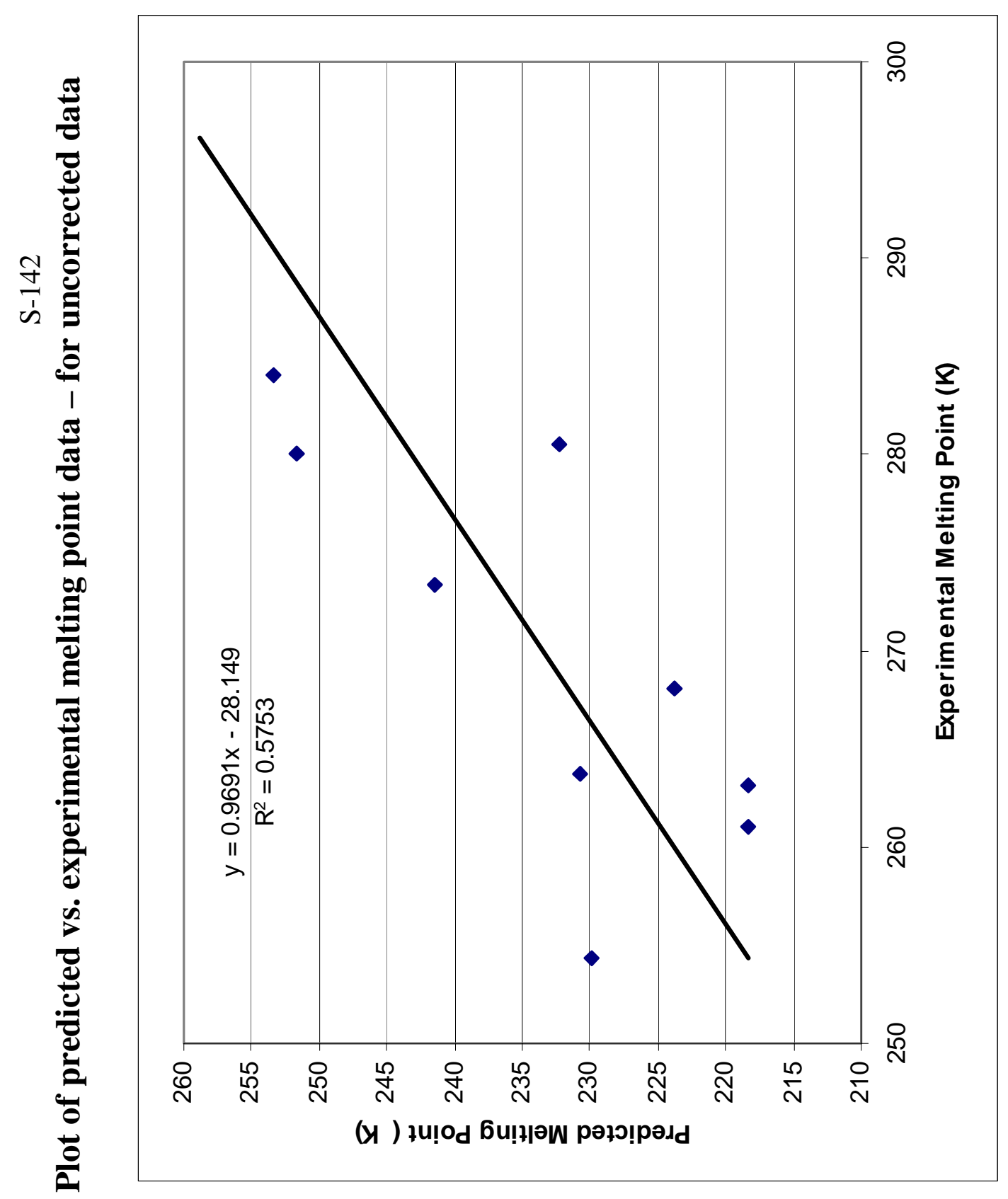




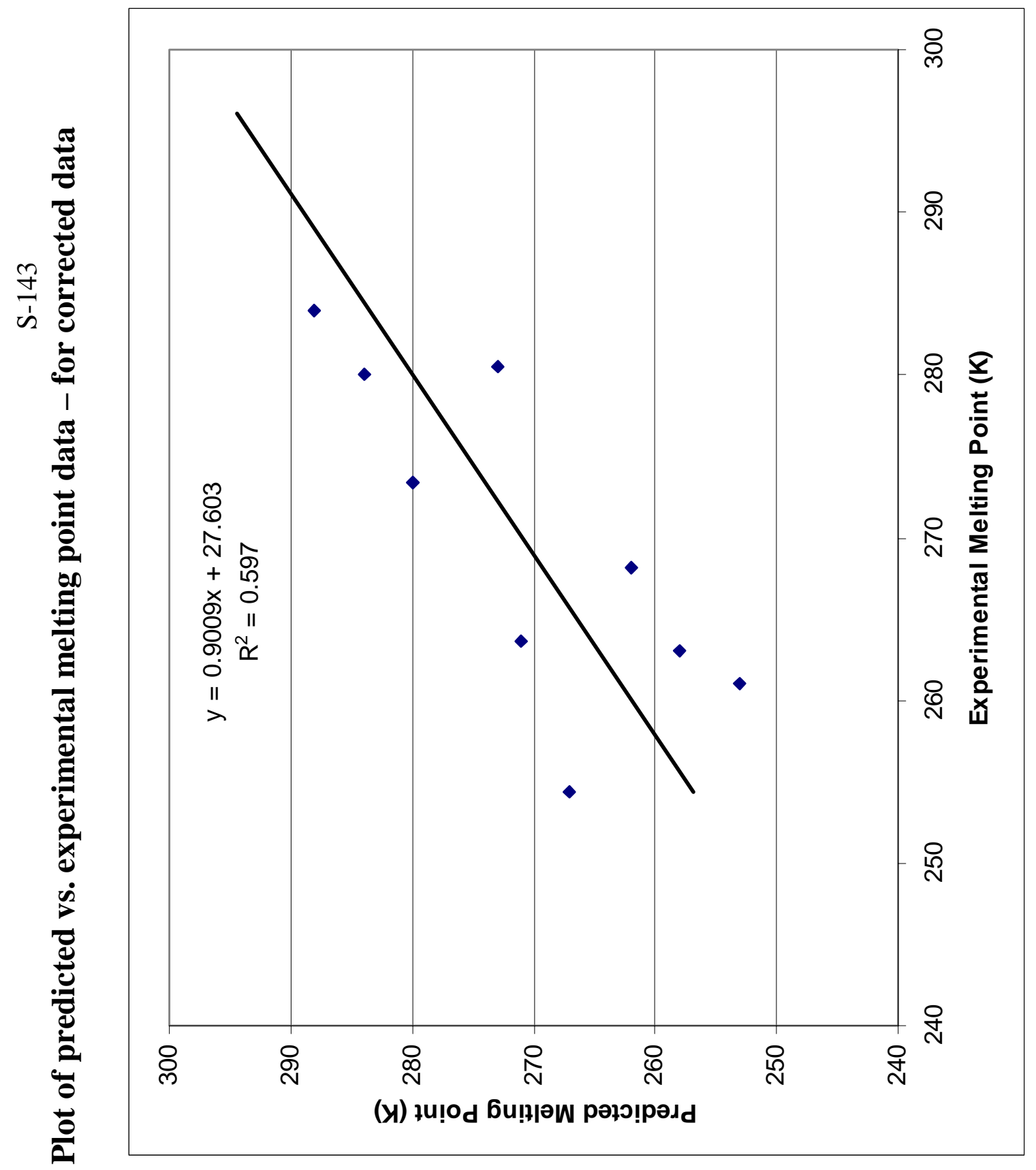



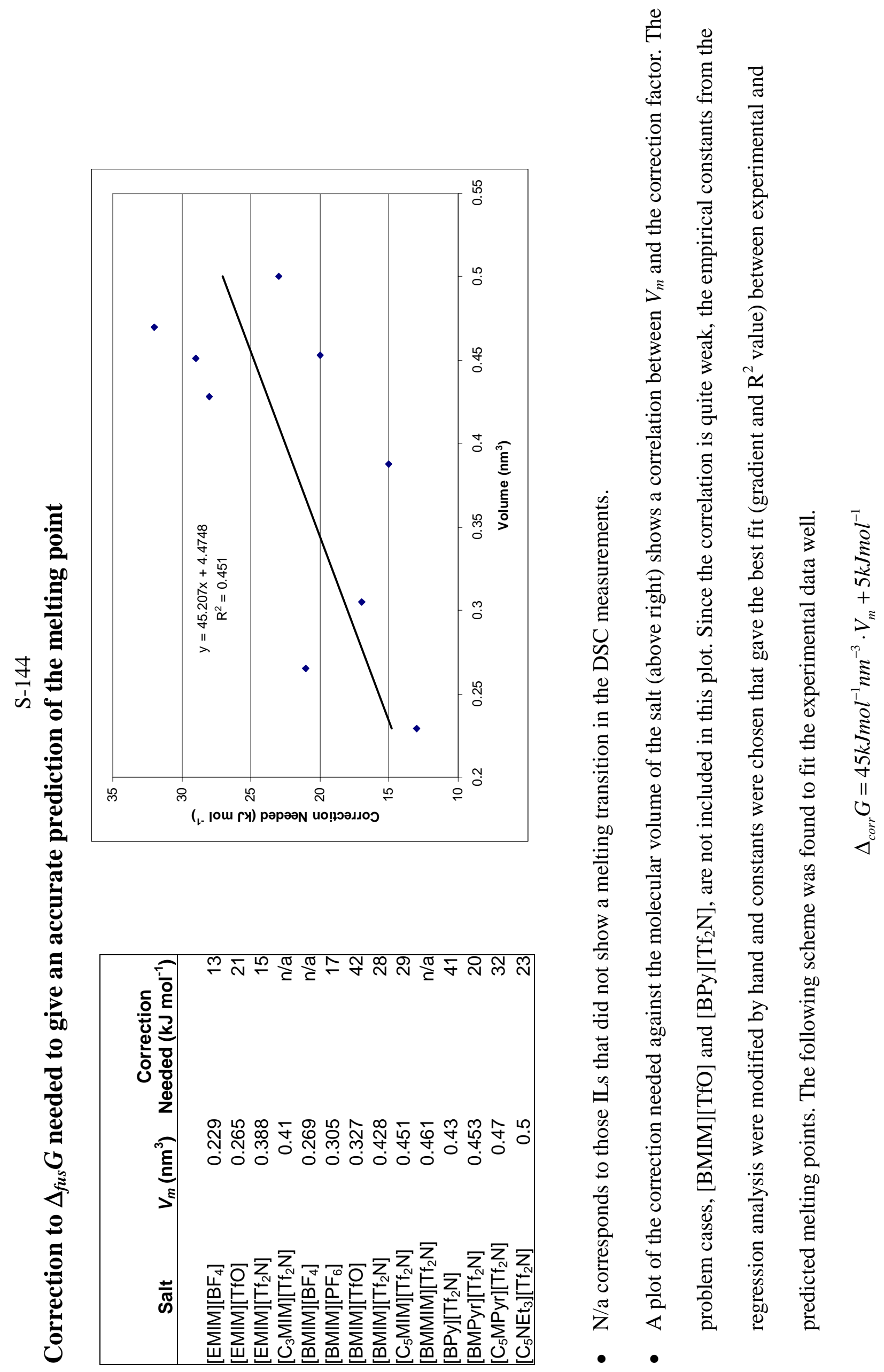
(') Cammarata, L.; Kazarian, S. G.; Salter, P. A.; Welton, T. Phys. Chem. Chem. Phys. 2001, 3, 5192.

(ii) Lancaster, N. L. ; Salter, P. A. ; Welton, T. ; Young, G. B. J. Org. Chem. 2002, 67, 8855.

(iii) Wasserscheid, P. ; Sesing, M. ; Korth, W. Green Chemistry, 2002, 4, 134.

$\left({ }^{\text {iv }}\right)$ Tokuda, H. ; Hayamizu K., Ishii, K ; Susan, M. A. B. H. ; Watanabe, M. J. Phys. Chem. B., 2005, 109, 6103.

( $\left.{ }^{v}\right)$ Ngo, H. L. ; LeCompte, K ; Hargens, L. ; McEwen, A. B. Thermochem. Acta 2000, 357-358, 97.

$\left(^{\mathrm{vi}}\right)$ Bonhôte, P.; Dias, A.-P.; Papageorgiou, N.; Kalyanasundaram, K.; Grätzel, M. Inorg. Chem. 1996, 35, 1168.

( ${ }^{\text {vii }) ~ W a k a i, ~ C . ; ~ O l e i n i k o v a, ~ A . ; ~ O t t, ~ M . ; ~ W e i n g a ̈ r t n e r, ~ H . ~ J . ~ P h y s . ~ C h e m . ~ B . ~ 2005, ~ 109, ~} 17028$.

(viii) Weingärtner, H.; Oleinikova, A.; Wakai, C.; Daguenet, C.; Dyson, P. J.; Krossing, I.; Slattery, J. M. J. Phys. Chem. B. 2006, 110, 12682-12688.

(ix) Hofmann, D. W. M. Acta. Cryst. 2002, B58, 489-493. 